\title{
Der Herausgeber
}

14 Nummern hat Prof. Dr. Horst Prießnitz herausgegeben und den Newsletter zu dem gemacht, was er ist: ein reichhaltiges, informatives Organ der Gesellschaft für Australienstudien, das auch einen Einblick in deren Leben gibt. Welche Veranstaltungen fanden statt, wo liegen die Interessen der Mitglieder, aus welchen Bereichen kommen sie? Mein Vorgänger hat dankenswerterweise für eine Kontinuität gesorgt: der Newsletter wird weiterhin an der Bergischen Universität GHS Wuppertal gedruckt und von dort versandt. Dafür sind wir ihm und der Universität zu Dank verpflichtet.

Der Newsletter wird weiterhin das Leben der GASt wiederspiegeln. Aber jeder Herausgeber wird versuchen, ihn noch ansprechender und informativer zu machen. Damit ist keine Akademisierung gemeint, auch wenn die Mehrzahl der Mitglieder aus den Hochschulen kommt. Aber viele kommen aus den Medien, den zoologischen Gärten, dem Museumsbereich, dem Kulturmanagement, der Wirtschaft oder internationalen Institutionen. Was kann der Herausgeber machen, um eine so vielfältige Leserschaft anzusprechen, zumal der Newsletter nur einmal pro Jahr erscheint?

Ich denke an dieses: Erstens werde ich für jedes Jahr einen Themenschwerpunkt setzen, der aus unterschiedlichen Gründen aktuell ist und aus vielen Perspektiven beleuchtet werden kann. Zweitens soll der Newsletter der Vielfalt der Mitglieder und Leser Raum geben, sich darin wieder zu erkennen und interessante Beiträge zu liefern. Da der Schwerpunkt andere Themen nicht ausschließt, finden sich in der Struktur auch die bekannten Sektionen wieder. Schließlich wird der Newsletter einen aktiveren Rezensionsteil und gelegentlich einen Resourcenteil—so zum Webenthalten, um auf wichtige Publikationen und Informationsquellen aufmerksam zu machen. Ich erwarte ein aktives Echo, wobei Autoren nicht nur aus dem Kreis der Gesellschaft kommen werden. Der Newsletter ist offen für Beiträge von außerhalb und aus Ländern, in denen Australien Gegenstand des öffentlichen und akademischen Interesses ist. Die Klagenfurter Konferenz hat gezeigt, daß wir-neben Australien-über Deutschland, Österreich und der Schweiz hinaus mit Kollegen aus den Ländern Mitteleuropas ebenso kooperieren sollen, wie mit denen aus Westeuropa.

Nun zu diesem Heft. Die Wahl eines Schwerpunktes war einfach, denn die Föderation der britischen Kolonien zum Commonwealth of Australia im Jahre 1901 ist das herausragende Datum. Der Newsletter beleuchtet dieses Ereignis aus der Sicht der Politik, Wirtschaft, Politik, Geschichte, Literatur und Sprache. Als Vorgabe für die Autoren wurde lediglich angeregt, auch auf Fragestellungen für das 3. Jahrtausend einzugehen oder darauf hinzuweisen.

\section{Last but not least der Schwerpunkt für Nummer 16, 2002 (Redaktionstermin 1. Juni 2002): "MOSAIK, MELTING POT UND SYNKRETISMUS. AUSTRALISCHER MULTIKULTURALISMUS IM 3. JAHRTAUSEND."}

Der Zusammenhang verschiedenet ethnischen oder anders definierter Gruppen eines multikulturellen Australien verdient eine Neubetrachtung. Zuviel ist seit den frühen 90er Jahren passiert. Ist Multikulturalismus noch das unbestrittene Gesellschaftsbild? Welche Alternativkonzepte haben sich herauskristallisiert? Wie spiegeln sich damit verbundene Themen in der Literatur, in Film und Fernsehen, in der Kunst wider? Welchen Status hat die ethnische Literatur? Wie reagiert die Sprachpolitik auf nichtenglischer Sprachen heute? Wie gehen Industrie, Handel und Politik mit Pluralisimus um? Inwieweit lenken staatliche Fördereinrichtungen (z.B. Arts Council, ATSIC, etc.) die Entwicklungen? Diese Fragen seien hier zur Anregung vorgegeben. 


\section{SCHWERPUNKT: FEDERATION 1901}

\section{Australien: eine Verfassung für einen Kontinent Susan Shineberg, freie Journalistin, Berlin}

Sonne, Traumstrände und Sport - das sind die Bilder, die die meisten Leute vor Augen haben, wenn von Australien die Rede ist. Seit den Olympischen Spielen von Sydney im September letzten Jahres verzeichnet Down Under einen Touristenboom. Der Reiz von Sonne, Surfparadiesen, atemberaubenden Landschaften und freundlichen Menschen beherrscht die allgemeine Vorstellung.

Aber wie steht es mit Australiens Geschichte? Wer weiß zum Beispiel, dass die noch junge australische Verfassung die geheime Wahl eingeführt hat, die in der englischsprachigen Welt noch heute "australische Wahl" heißt? Oder dass es das zweite Land (nach Neuseeland) war, das Frauen das Wahlrecht eingeräumt hat?

Der fünfte Kontinent, diese riesige Landmasse in der südlichen Hemisphäre, hätte leicht auch zu einer ganz anderen politischen Konstellation kommen können. Selbst vielen Australier ist nicht bewußt, wie nahe das Land davor stand, sich überhaupt nicht zu einigen. Ohne die Bewegung der Föderalisten und ihre hohen politischen Ideale würden wir heute vielleicht auf einen Kontinent mit drei oder sogar fünf verschiedenen Nationen blicken. Um die volle Bedeutung dieser Bewegung zu verstehen, müssen wir zurückblicken auf die Geschichte des Landes.

Zehntausende von Jahren vor den ersten europäischen Siedlungen in Australien gehörte der riesige Kontinent allein der indigenen Bevölkerung. 1788 gründete Großbritannien dort eine Strafkolonie als Teil seines ausgedehnten Weltreiches. Im Gefolge der Sträflinge und Militärs kamen freie Siedler, die zunächst die fruchtbaren Küstenregionen besiedelten und dann ins Landesinnere vordrangen. Viele kamen in den 1850er Jahren auf der Suche nach Gold, einer Zeit, in der Australien einen großen Bevölkerungszuwachs verzeichnete. Zwei Drittel der Landmasse des Kontinents wurde zur "Britischen Kronkolonie von Neusüdwales" erklärt und umfasste die heutigen Staaten Tasmanien, Queensland, Victoria und New South Wales - Ort der ersten Besiedlung. Zwei andere Staaten, Westaustralien und Südaustralien, wurden als vollkommen eigenständige Kolonien gegründet. Erst 1851 fand die offizielle Teilung Australiens in sechs verschiedene Kolonien statt, denen Großbritannien jeweils eine eingeschränkte Form der Selbstverwaltung zugestand - womit Australien tatsächlich erst begann, eine Nation zu werden.

Auch wenn eine gemeinsame Sprache und Kultur die Kolonien deutlich miteinander verband, standen die wirtschaftlichen Interessen der winzigen, über den ganzen Kontinent verstreuten britischen Vorposten oft im Widerspruch zueinander. Tiefsitzendes Misstrauen und offene Feindseligkeit zwischen den einzelnen Kolonien erwiesen sich als schwerwiegende Hindernisse bei der Frage nach einer Einigung, gemeinsames Erbe hin oder her - gerade Deutsche werden dies nur allzu gut verstehen.

Die größten Spannungen bestanden erwartungsgemäß zwischen den beiden größten Kolonien. New South Wales mit seinem rauheren und offeneren Zentrum Sydney (dem Ort der ersten Strafkolonie), und Victoria, dessen Hauptstadt Melbourne sich selbst als sehr viel zivilisierter betrachtete, waren zwei große Rivalen. Hauptgrund der Differenzen und größtes Hindernis für eine Einigung war ihre Haltung zum Zollrecht und den damit verbundenen Einnahmen. Neusüdwales mit seiner landwirtschaftlich ausgerichteten Wirtschaft sprach sich für den Freihandel aus. Victoria dagegen wo das herstellende Gewerbe (finanziert vom Geld der Goldgräber) eine größere Rolle spielte verfolgte eine protektionistische Politik.

Dieser Interessenkonflikt nahm bald ideologische und moralische Dimensionen an und konnte trotz mehrfacher Versuche erst beigelegt werden, als sich die Kolonien zu einer Föderation zusammenschlossen. Beispielsweise existierte bereits in den 1860er Jahren ein Plan für eine 
Zollunion nach dem Modell des deutschen Zollvereins von 1833. Auf diesem soliden Fundament hätte später eine Föderation stehen können, wie dies in Deutschland 1871 der Fall war. Die verschiedenen kolonialen Handelskammern jedoch hatten allein ihre Handelsinteressen im Auge und konnten zu keiner Einigung über weiterreichende wirtschaftliche Interessen gelangen, die von einer Einigung profitieren würden.

Auf der anderen Seite kamen die Kolonialregierungen auch allein überraschend gut zurecht. Und in den nachfolgenden Jahrzehnten entstand in vielen wichtigen Bereichen eine Zusammenarbeit zwischen den Kolonien. Große Geschäfte wurden über die Grenzen der Kolonien hinweg abgeschlossen, und Arbeiter konnten sich problemlos dorthin begeben, wo sie gerade am meisten gebraucht wurden. Kirchen, Gewerkschaften und Berufsgenossenschaften unterhielten vielfach grenzüberschreitende Verbindungen. Um 1880 operierten Post und Telekommunikation, Eisenbahnen, Einwanderungs- und Verteidigungspolitik auf nationaler Ebene.

Was also trieb die sechs Mini-Regierungen zur Einigung? Trotz des Reizthemas fortbestehender Handelshemmnisse gab es keinen drängenden Grund bestand, eine Nation zu bilden. Und wenn man die deutschen und französischen Ambitionen im Pazifik auch mit einer gewissen Sorge betrachtete, gab es doch keine direkte äußere Bedrohung - einer der häufigsten und zwingendsten Gründe für die Entstehung einer Föderation.

Die jüngsten Bemühungen der Europäischen Union, einen Konsens bei ihren Zuständigkeitsbereichen zu erreichen, machen nur allzu deutlich, wie wenig Staaten im allgemeinen bereit sind, Selbstbestimmungsrechte abzutreten. Im Falle der EU ist der Versuch, gesetzliche und politische Strukturen zu installieren, weitreichenden wirtschaftlichen Übereinkommen gefolgt. In Australien war es umgekehrt.

Sie gewann in den 1880er Jahren Auftrieb, als der dynamische Politiker Henry Parkes, Premier in New South Wales, eine leidenschaftliche Ein-Mann-Kampagne für die Einigung aller sechs unabhängigen Kolonien zu einem Commonwealth of Australia ins Leben rief. Er überzeugte Delegierte aus allen Kolonien, an der Versammlung von Sydney im Jahre 1891 teilzunehmen und einer Verfassung zuzustimmen. Auch wenn dieser ursprüngliche Plan erfolglos blieb, entstand 1893 eine breiter angelegte Föderationsbewegung, durch die zum ersten Mal die Menschen in den Kolonien - und nicht die Parlamentsmitglieder - aufgefordert wurden, eine neue Versammlung zu wählen. Es galt, eine Verfassung zu entwerfen und in einem Referendum zur Abstimmung zu bringen. Dieser Mechanismus kam 1897 zur Anwendung, und eine Verfassung wurde schließlich von allen akzeptiert. Aber sie war noch nichts Endgültiges. Queensland und Westaustralien akzeptierten die Bedingungen nur knapp.

Den Aborigines, die vorher in einigen Staaten gewählt hatten, wurde kein Wahlrecht zugesprochen, während Frauen es erhielten. Konservative in Queensland und Westaustralien fürchteten die große Zahl indigener Einwohner in ihren Staaten, wodurch die Föderalisten sich gezwungen sahen, Konzessionen zu machen, um diese Kolonien zur Beteiligung am Einigungsprozess zu bewegen. Darüber hinaus zeigten sich die Labor-Politiker besorgt, die Stimmen der Aborigines in den Gebieten des Outbacks könnten von großen Landbesitzern kontrolliert werden. Die LaborBewegung stand ebenfalls an der Spitze der sogenannten "Politik des Weißen Australien", die darauf abzielte, Arbeitsimmigranten aus China und sogenannte "Kanakas" von den Pazifikinseln aus dem Land zu halten. Zu bestimmten Zeiten galt dies als "progressiv", als ein Mittel, den Arbeiter und die "Würde der Arbeit" zu schützen. Tatsächlich ging es darum, die Arbeiter vor den billigen "farbigen" Arbeitskräften zu schützen, die die Plantagenbesitzern bevorzugten - eine Angst, die durch die Nähe Asiens genährt wurde. Die dunkle Wolke am politischen Horizont Australiens, die damals entstand, sollte die Nation noch lange verfolgen.

Trotz dieser Unzulänglichkeiten war das, was schließlich entstand - festgeschrieben in der Verfassung des Landes - eine der ersten progressiven, liberalen Demokratien. Im Jahre 1900 wurde die Verfassung vom britischen Parlament verabschiedet und trat am 1. Januar 1901 in Kraft; zwei 
Monate später fanden die ersten landesweiten Wahlen statt. Zweifellos bildete in weiten Teilen die britische Westminster-Demokratie die Grundlage, modifiziert durch die Erfahrung Amerikas. Doch machten heimische Zusätze sie zu einer Besonderheit, ebenso wie die Entschlossenheit, ihr einen Sinn für Gleichheit einzuflößen, Klassenunterschiede aufzuheben und soziale Schranken abzubauen. Diese Werte, die dazu beitrugen, eine offene und menschenfreundliche Gesellschaft zu schaffen, haben sich bis heute bewährt. 


\section{Australia's Federation- from an Inidgenous Australian's perspective Eve Mumewa D. Fesl, Assoc. Professor, OAM., Ph.D., Elder of the Gubbi Gubbi and Gangulu Peoples.}

This year non-Indigenous Australians celebrate "Federation", the year in which they achieved nationhood, albeit under English control.

Is this a 100-year celebration for we, the Indigenous people? Certainly not!

As if invading our country and stealing our lands wasn't enough, in 1897 what became known as "The Protection" Act, was enacted, legalizing an ad hoc policy of snatching our children their mothers' arms then raising them in institutions without giving them information as to who their parents were (Nor were parents told where their children had been taken). ${ }^{1}$ The Act empowered police to enforce our people at gunpoint into missions and reserves hundreds of kilometers from their spiritual lands. There slavery began under the euphemism "christianizing and civilizing". 2

This Act was in full swing as non-Indigenous Australia raised its flag. During the celebrations my people were being moved from their homelands to institutions where conditions were far worse than prison-farms today. But my people had committed no crimes - my Grandmother, Uncles and Aunts died there. My mother now 92 years old remembers the day clearly when she was imprisoned with them. ${ }^{3}$ She can still hear their cries and see their tears as they wept for the country, the spiritual base from which they were removed - never to be allowed to return except by stealth for a brief visit.

Activities under the Act continued until circa 1975 in various States of Australia. In 1967 it had been decided we were to be counted in a census of the population, but we were not given the right to vote in elections under the same conditions as other Australians until 1985.

One can perceive some improvement in the last 16 years - perhaps this is a cause for celebration. A process of reconciliation had begun in 1990 (but the money to educate non-Indigenous people about the untold contact history they had catalyzed, had to be paid for out of our budget - $\$ 40$ million - a sum which would have helped improve living conditions and health for many of our people

As conditions for us begin to improve Australia is benefiting. Work by our artists brings in important export dollars, the few of our athletes who have had an opportunity have performed well at the international level, some of us have managed to achieve well academically. To do these things we have had to work harder than most non-Indigenous people. Covert racism exists in many institutions. People with power do not relinquish it willingly or easily "divide-and-rule games" seem to be the order of the day. As long as our people remain poor there will always be someone who can be "bought". It is therefore in the interests of the power hungry to ensure that economic progress for us is discouraged.

As I was writing this article I received a telephone call from a distressed woman. She was one of the children abducted under the Protection Act - she was still trying to trace her family to, as she said "find out who I am".

Daily tragic memories of the past 100 years are re-enacted throughout our country. After that call I was reticent about joining in the "Federation Trails". However, this time my people will be at the forefront of many celebrations across the nation. I don't want us to be forgotten again. There is a glimmer of light at the end of the tunnel.

\footnotetext{
1 "The Aboriginals Protection and Restriction of the Sale of Opium Act 1897-1901". There were later amendments.

${ }^{2}$ To avoid detection by the Exeter Hall Movement which had banned slavery throughout the world in 1830.

${ }^{3}$ As a consequence of being slaves and having no money and nowhere to go, when restrictions were lifted, our family, immediate and extended, has been poor ever since.
} 
I will join in the celebrations for my people, to honor the SPIRITS OF OUR LAND and to PAY HOMAGE TO MY ANCESTORS who suffered so much but left me with a legacy of courage and strength to survive. In other words I will celebrate OUR SURVIVAL! 


\section{Das zögerliche Zusammenwachsen einer Nation: \\ Der West Australian von 1900-1933 \\ Dr. Beate Josephi, Edith-Cowan University, Perth}

\section{Fragestellung}

Oft erweckt die Erwähnung der 'Australian Federation' den Eindruck, als sei mit dem Zusammenschluss der australischen Kolonien zum Commonwealth von Australien glockenschlagartig eine neue Nation mit einem gereiften Selbstverständnis geboren worden. Die vorliegende Studie will diesen Eindruck korrigieren, indem sie der Frage nachgeht, inwieweit an Inhalt und Redaktionswesen des West Australian Veränderungen von einer britisch-kolonialen zu einer australischen Zeitung in den ersten 33 Jahren nach der Föderation zu beobachten sind.

Die westaustralische Tageszeitung The West Australian, 1833 gegründet und damit nach dem Sydney Morning Herald Australiens zweitälteste Zeitung, wurde wie fast alle australischen Zeitungen von den Konventionen des britischen Journalismus geprägt (Cryle 1997:15). Allerdings mussten die britischen Pressetraditionen einem Land mit einer völlig anderen Geographie und einem anderen Siedlungmuster angepasst werden (Lloyd 1999:11).

Das galt vor allem für Westaustralien, das ein Drittel des Kontinents einnimmt und damit sieben mal so gross wie die Bundesrepublik Deutschland ist, aber im Dezember 1884, ehe die Goldlager um Kalgoorlie entdeckt wurden, nur 33.000 Einwohner zählte (Crowley/de Garis 1969:26). In Crowleys Worten war Westaustralien "the most British of all Australian colonies and was governed by a group of families who looked westward towards England rather than to the East" (Crowley 1960:110). Im späten 19. Jahrhundert mussten sich die Westaustralier in allererster Linie der Herausforderung der "tyranny of distance" stellen (Blainey 1966:VIII/IX). Paul Kelly nannte die Föderation daher "a unique event in world history: never before had such small communities divided by such distances voluntarily decided to form a new nation" (Kelly 2001:1).

Auch wenn die Zeitungsstruktur in britischen Traditionen wurzelte, wurden die Blätter von Nachrichten und Berichten örtlichen Inhalts gefüllt, was die Struktur überlagerte und allmählich einen lokalen Anstrich gab. Die vorliegende Studie beschäftigt sich daher sowohl mit Inhalt wie auch dem Redaktionswesen. Die Inhaltsanalyse wurde von qualitiativen Gesichtspunkten geleitet, da eine quantitative Studie im Rahmen dieser Arbeit nicht möglich war. Hierbei wurden in erster Linie Leitartikel, aber auch andere Berichte an signifikanten Daten herangezogen. Sie beginnt in den Tagen vor der westaustralischen Abstimmung zur Föderation, dem 31 Juli 1900, und endet am 10 April 1933, dem Tag, an dem das Ergebnis des westaustralischen Sezessionsreferendums veröffentlicht wurde. Nahezu doppelt so viele Westaustralier hatten am 8 April 1933 für die Sezession vom Commonwealth of Australia gestimmt wie dagegen. Doch da dieser Schritt von der Zustimmung der anderen australischen Staaten abhing, wurde das Votum zur verfassungsrechtlichen Wirklichkeit. Allerdings bietet dieser Wahlausgang eine bedeutsame Teilantwort auf die Frage, ob die Föderation wirklich als der Moment angesehen werden kann, an dem der West Australian zu einer von nationalem australischen Bewusstsein getragenen Zeitung wurde.

\section{Der Zwiespalt: ' Empire or Nation?'}

Der hundertste Jahrestag der Föderation war Anlass zu einer Reihe von Veröffentlichungen, die sich mit den geschichtlichen Ereignissen um diesen Zeitpunkt der Föderation herum beschäftigen oder, wie Paul Kellys der ABC Fernsehserie zugrunde liegendem Buch, 100 years - The Australian Story, sich mit den ersten hundert Jahren der australischen Geschichte auseinandersetzen. Kellys Studie erinnert vor allem daran, wie zögernd sich Australien - zumindest bis zum zweiten Weltkrieg - von Grossbritannien löste. Hierin bietet Kellys Buch ein Gegengewicht zu früheren Arbeiten, die vor allem das nationale Zusammenwachsen, das 'making it national' betonten (Turner 1994). Kelly 
schreibt, "The fusion of nationalism and Empire, now long gone, is often denied" und führt an, dass Schriftsteller und Historiker von Henry Lawson über Russell Ward bis Manning Clark in ihrem Versuch "to find Australia a more lofty purpose in ideals of nationalism, mateship and democracy", das wirkliche Ausmass der konservativen Elemente und der britischen Tradition in Australien überblickt haben (Kelly 2001:7).

Wie Geschichtsschreibung hat auch Zeitungsgeschichte die Tendenz, das Werden einer Nation zu betonen. Rantanen, Schlesinger zitierend, hebt hervor, dass "media histories in general have an overarching interest in showing how media institutions contribute to the shaping of national culture, economy and polity" (Rantanen 1997:607). Das trifft auch auf den klassischen Text zur Bedeutung von Zeitungen beim Entstehen einer Nation zu, Benedict Andersons Imagined Communities. Anderson unterstreicht hierin die Rolle von Zeitungen und anderen Schriftmaterialien als einzigartige Sammelstellen und Fokalpunkte für das sich entwickelnde Nationalbewusstsein, was besonders im 19. Jahrhundert in sprachlich vom Mutterland getrennten Teilen eines Reiches - wie zum Beispiel Teilen der k.u.k. Monarchie - nachzuvollziehen ist. Laut Anderson weist dies auf eine "inner incompatibility of empire and nation" hin (Anderson 1983/1991:93). Für Kelly besteht eine solche Unvereinbarkeit nicht. Nach seinen Worten lag die australische Idee in einer Synthese "of indigenous nationalism and Empire loyalty. ... This meant that an imperialist could be a nationalist and a nationalist could champion the Empire" (Kelly 2001:3).

Wenn man diese beiden Szenarien am West Australian misst, erscheint Kellys als das akkuratere. Um die Jahrhundertwende gaben die im Medienspektrum als konservativ geltenden Morgenzeitungen der Hauptstädte - zu denen auch der West Australian zählt (de Garis 1981:336) sehr viel eher der Begeisterung für das Empire Ausdruck als für die junge australische Nation. Wie auch Kirkpatrick, H.M. Green zitierend, hervorhebt, "The conservatism of the leading city papers kept them ... in the rear of the democratic tendencies of the age; the classes they represented were the last to feel the influence of the new Australianism" (Kirkpatrick 2000:80; s. Lawson 1999:89). Dies stimmt mit dem von Kelly hervorgehobenen Punkt überein, dass die neue Nation ein vom Mutterland initiierter und unterstützter wirtschaftlicher und rechtlicher Zusammenschluss war und noch keine - zum Beispiel durch einen Freiheitskampf gewachsene - emotionelle und geistige Einheit.

In diesem Zusammenhang muss man sich auch vergegenwärtigen, dass Australien bis 1953 nicht seine eigene Fahne benutzte und dass "God Save The King" bzw. "God Save The Queen" bis 1974 auch die australische Nationalhymne war. Erst seit 1984 wurde "Advance Australia Fair" bei allen öffentlichen Anlässen gespielt, ausser in der Gegenwart des Monarchen, und erst 1986, mit der Verabschiedung der 'Australia Acts', wurden die letzten kolonialrechtlichen Verbindungen mit Grossbritannien gelöst.

\section{Westaustralien, seine Kommunikationslinien und der West Australian um 1900}

Westaustralien war die letzte der Kolonien, die über den Zutritt zur Föderation abstimmte. Wie der damalige Spitzname der 'Cinderella Colony' besagt (Crowley/de Garis 1969:26), blühte Westaustralien erst später als die Staaten der Ostküste auf, aber behielt das früh geprägte Verhältnis von geringer Einwohnerzahl und grossem Exportanteil bei. An der Wende zum 21. Jahrhundert erwirtschaftete Westaustralien mit weniger als $10 \%$ der Bevölkerung nahezu ein Viertel des Bruttosozialprodukts. Es darf daher nicht wundern, dass der Ruf nach einer westaustralischen Sezession bis heute nie ganz verklang. Selbst Anfang der neunziger Jahre des 19. Jahrhunderts war die "newly independent colony ... the only safe investment among all the Australian colonies. Its financial position was sound, its population increasing, its agricultural and pastoral areas showed promise of a brighter future and, above all else, its goldmining industry was beginning to attract world-wide attention" (Crowley 1960:110).

Westaustralien wurde 1890 eine selbstständige Kolonie und Ministerpräsident während des Jahrzehnts bis zur Föderation war Sir John Forrest, den man einen 'reluctant federalist' nannte. Die 
Abstimmung über den Beitritt zum australischen Commonwealth fand am 31 Juli 1900 statt. Der Wahlausgang zeigte eine grosse Mehrheit für den Beitritt - 44.652 stimmten dafür und 19.636 dagegen. Er wurde besonders in den Goldfeldern um Kalgoorlie - deren Bewohner überwiegend aus den Oststaaten kamen - und in Perth unterstützt, während die Farmgebiete des Südwestens gegen den Beitritt stimmten (Crowley/de Garis 1969:54).

Im Vorfeld der Abstimmung unterstützte der West Australian nur halbherzig den Vorschlag der Föderation, schwenkte dann aber immer mehr auf den Kurs für den Zusammenschluss ein. Die beiden Eigentümer der Zeitung, Charles Harper und Winthrop Hackett, waren sich in ihrer Haltung nicht einig, was sie am Tag des Votums in einem Artikel mit der Überschrift "News and Notes" darlegten: "We are requested to state that the responsibility for the advocacy of Federation rests with the Editor of the 'West Australian' [Hackett]. This statement is necessary in view of the fact that Mr. Harper, one of the chief proprietors of this journal, is warmly opposed to that movement, believing its effects will be seriously injurious to the colony." Daher solle die Zeitung so weit wie möglich eine neutrale Haltung bewahren ("News and Notes", The West Australian, 31.1.1900, p. 4). Auf die Tatsache, dass dies in den Tagen vor der Wahl lediglich ein frommer Wunsch war, wird weiter unten eingegangen werden.

Kommunikationslinien - in diesem Falle die transkontinentale Eisenbahn - waren eines der Projekte, die einer der Herausgeber und Chefredakteur des West Australian, Winthrop Hackett, gefährdet sah, sollte Westaustralien nicht der Föderation beitreten. Allerdings beleuchteten die telegraphischen Verbindungslinien sehr viel deutlicher die komplexe Verkettung des britischen Empires und der daraus entstehenden Nationen.

Alle australischen Kolonien ausser Westaustralien hatten 1872 eine telegraphische Verbindung zu England hergestellt, als das Unterseekabel der im privaten Besitz befindlichen, aber von öffentlichen Geldern unterstützten Eastern Extension Company in Port Darwin an Land gezogen und von dort über Land nach Adelaide und weiter zu den Ostküstestaaten geleitet wurde. Die Telegraphenlinie nach Perth wurde jedoch erst 1877 fertiggestellt. Laut Putnis beinhalteten diese neuen Kommunikationswege eine grosse Symbolkraft. "The emotional bonds of imperial sentiment and loyalty could hereby be materially realised. ... In the case of Australia it could be said that the modern technology of the telegraph reconstituted and reinvigorated a pre-existing imperial imaginary" (Putnis 1999:141).

Die Agentur Reuters versuchte, die Monopolstellung als einzige Nachrichtenquelle zwischen Australien und der Welt zu erlangen, indem es vorschlug, die Nachrichten in London zentral zu sammeln und die jeweilige Selektion weiterzuleiten. Doch hier spielten die australischen Zeitungen nicht mit. Mitte der 80iger Jahre des 19. Jahrhunderts hatten sich zwei Syndikatsgruppen gebildet, die Argus Gruppe - der der Sydney Morning Herald und später auch der West Australian angehörte - und die Gruppe um die Melbourner Age. Sie eröffneten ihr eigenes Büro in London, wo ihre eigenen Angestellten die nach Australien zu telegraphierenden Nachrichten aus dem dort sehr viel breiter gefächerten Reuters Angebot auswählten (Harcourt 1987:66; Putnis 1999:146). 1895 schlossen sich die beiden Gruppen unter dem Melbourner Argus zusammen, aber bezogen ihre Nachrichten noch immer hauptsächlich von Reuters. Dieses Monopol besorgte die neue australische Bundesregierung so sehr, dass sie 1909 eine Senatsanhörung einberaumte. Bezeichnenderweise wurde dies auch damit begründet, dass die bestehende Übermittlungsstruktur nicht genügend Nachrichten aus anderen Teilen des Empire biete (Putnis 1999:153).

Die Sorge um ungenügende Verbindung zwischen den Teilen des Empires zeigt deutlich die Prioritäten der australischen Regierung auf. Es ging ihr nicht um die eigene Kontrolle der Nachrichtenverbindunglinien, sondern in erster Linie um das Empire und seinen Zusammenhalt. Dies stimmt mit Kellys Behauptung überein, dass Australien "became a self-governing nation within the Empire, not an independent country as the term is known today" (Kelly 2001:4). 
The West Australian ist ein weiterer Beweis einer solchen nahtlosen Verschmelzung. Um die Jahrhundertwende gehörte die Zeitung Charles Harper und Winthrop Hackett. Beide waren zugleich Parlamentarier und schon vor 1900 hatte Harper Hackett die redaktionelle Leitung der Zeitung überlassen.

Winthrop Hackett, ein gebürtiger Anglo-Ire, der am Trinity College in Dublin Jura studiert hatte, war 27jährig nach Australien ausgewandert. 1883 nahm er eine Stelle beim West Australian an und wurde 1887 Chefredakteur. Als Westaustralien eine unabhängige Kolonie wurde, bot Ministerpräsident Forrest Hackett ein Ministerium an, was dieser ausschlug; er wollte lieber weiter hinter den Kulissen Berater Forrests und vor den Kulissen Sprachrohr der Regierung war. Hackett unterstützte das Wahlrecht für Frauen und setzte sich erfolgreich für die Schulpflicht und die Einführung schulgeldfreier Staatsschulen ein, wie auch für die Gründung der Universität von Westaustralien (Hunt 1983:152). Anders als andere Zeitungsbesitzer in dieser Zeit (Lloyd 1999:16) gründete Hackett keine Mediendynastie. ${ }^{1}$ Als Berater des Ministerpräsidenten, Politiker und Chefredakteur spielte Winthrop Hackett eine zentrale Rolle in Westaustraliens Diskussion um den Beitritt zur Föderation. Aber er entschied sich erst sehr spät - im Januar 1900 - diesen Schritt zu unterstützen (Hunt 1983:152).

Dadurch, dass beide Besitzer aktiv in der Politik waren, fiel ein Grossteil der redaktionellen Leitung Alfred Langler zu, einem Journalist, der aus Devon eingewandert war. Er trat 1895 dem West Australian bei und wurde 1902 stellvertretender Chefredakteur und Leitartikelschreiber. Nachdem Harper 1912 und Hackett 1916 starben, wurde Langler 1917 Herausgeber, Chefredakteur und Verwalter von Hacketts Erbe, das dieser tiefverschuldet hinterlassen hatte (The West Australian, 17.11.1979, p. 39). Langler führte diese Rollen selbstaufopfernd und finanziell erfolgreich aus und überraschte alle 1926 mit der Nachricht, dass "The West Australian's value was equal to his [Hackett's] bequest" (Richards 1989), und dass Hacketts Vermächtnis an die Universität und die anglikanische Kirche ausgezahlt werden konnte. Anders als Hackett was Langler in erster Linie ein Journalist "with a detachment from Australian politics" (Porter 1983:667). Langler trat 1927 in Ruhestand und starb ein Jahr später. Herbert James Lambert, ein gebürtiger Australier, übernahm die Redaktion von 1927 bis 1946.

\section{The West Australian zwischen Empire und Nation 1900/1901}

Hacketts Zögern, den westaustralischen Beitritt zur Föderation zu unterstützen, war nicht von einer Verbundenheit zu Grossbritannien oder einer Abneigung gegen Australien getragen. Argumente dieser Art lagen der Debatte völlig fern, da die Abstimmung nicht unter diesen Vorzeichen gesehen wurde. Der Beitritt wurde fast ausschliesslich als wirtschaftliche Entscheidung diskutiert, ob sich Westaustraliens Zukunft günstiger in einem Commonwealth of Australia gestalte, oder der Beitritt erst zu einem späteren Zeitpunkt—oder auch gar nicht—vollzogen werden solle. Hinzu kam die Sorge, dass sich Westaustralien spalten könne, da die Goldfelder um Kalgoorlie mit ihren hauptsächlich aus den Oststaaten kommenden Einwohnern für den Beitritt stimmen würden. Diese Gefahr wurde in der Zeitung hochgespielt, indem berichtet wurde, dass eine solche Abspaltung "would be strongly endorsed by [Secretary of State, Joseph] Chamberlain, and warmly supported by both parties in the Imperial Parliament" ('Editorial', The West Australian, 30.7.1900).

Selbst wenn Hackett, wie auch der Ministerpräsident, nur 'zaudernde Föderalisten' waren, machte die Zeitung am Vortag der Wahl ihren ganzen Einfluss für den Beitritt geltend. Unter der Überschrift "Federation for Western Australia" und einem Zitat aus Shakespeares Heinrich VI, "Join we together for the people's good", zählte der West Australian in einer drei Spalten einnehmenden Liste über 50 Gründe auf, warum Westaustralier in der morgigen Wahl mit Ja stimmen sollten. Fast alle waren wirtschaftliche Argumente, lediglich eines kann man als Ausdruck von Nationalgefühl ansehen: "Federation means Government of Australia by the Australian people."

\footnotetext{
${ }^{1}$ Sein Sohn General Sir John Hackett war von 1966-68 NATO General der Heeresgruppe Nord und gleichzeitig Oberbefehlshaber der britischen Rheinarmee.
} 
Die in der gleichen Ausgabe zitierte Rede des Ministerpräsidenten Forrest - eine Rede, die er zwei Jahre zuvor gehalten hatte - schlug einen anderen Ton an. Unter der Überschrift "Our Premier's Voice" begann sie mit den Worten, "We must not forget that the great minds in the mother country and in Australia desire this Federation" ("Our Premier's Voice", The West Australian, 30.7.1900, p. 4). Forrests Rede war eine von mehreren, die abgedruckt waren, darunter auch von Hackett. Hackett schrieb, "It seems to me that Western Australia can only use her position - the finest and most permanent asset she possesses - by combining with all Australia, to lift her into true and coming importance. The colony which commands the Indian Ocean, which lies nearest to China, India and South Africa, must have one of the greatest futures if it is not lost by the blunders of selfishness" ("Unity", The West Australian, 30.7.1900, p. 4). Dies war keine unbedingte Bestätigung des Commonwealth of Australia, sondern, wie es sich für den Herausgeber des West Australian gehörte, eine Rückenstärkung Westaustraliens.

Am Tag der Abstimmung, dem 31. Juli 1900, druckte die Zeitung die bereits erwähnte Notiz ihres anderen Besitzers, Mr. Harper, der sich wärmstens der Föderation entgegenstellte und sich wünschte, dass die Zeitung "should preserve an attitude as far as possible of neutrality". Die Ausgabe am Vortag widersprach diesem Wunsch aufs deutlichste. Am. 31. Juli 1900 erschienen die Nachrichten aus NSW, Queensland, South Australia, Tasmania und Victoria im West Australian zum letzten Mal unter der Sammelüberschrift "Intercolonial".

Das Votum fiel, wie erwartet, mit überwältigender Mehrheit zugunsten der Föderation aus. Am 1. August 1900 wurden die Wahlergebnisse wie auch Interviews mit führenden westaustralischen Politikern veröffentlicht. Diese reichten von den gemessenen Worten des Ministerpräsidenten, "I trust that all will unite in doing their best to promote not only the interests of Western Australia but the interests of Australia and the Empire" $\mathrm{zu}$ den euphorischeren Rufen des Federal League Präsidenten, Mr. Matheson: "From the Indian to the Pacific Ocean Australia is now one in hope and name and destiny." Aus diesen Worten wird klar, dass Australien nicht auf eine Vergangenheit zurückgreifen konnte, aus der ein Einigkeitsgefühl heraufbeschworen werden konnte, sondern dass man, nun geeint, auf die Zukunft setzte, die, in den Worten eines anderen Föderalisten, eine glorreiche sein könnte ("Interviews", The West Australian, 1.8.1900, p. 4).

Fast genau ein Jahr später war Perth die letzte Station auf der Reise des Duke und der Duchess of Cornwall, die nach Australien gekommen waren, um als Repräsentanten Ihrer Majestät den Föderationsfeierlichkeiten in Melbourne beizuwohnen. Die Leitartikel erwähnten die neue Nation kaum, sondern erwogen vielmehr, ob und wie Westaustralien das königliche Paar beeindrucken würde. Man bedauerte, dass die Hoheiten nicht die Zeit hatten, die Goldfelder zu besuchen. "They would there have seen the most striking examples of that auriferous wealth which, so long hidden in the desert, has raised Western Australia almost from penury to a condition approaching affluence, from insignificance to a position of promise." Dabei verglich sich Westaustralien nicht mit den anderen australischen Staaten, sondern mit anderen Teilen des Empires und nahm an, dass "when the Royal travelers proceed to South Africa, coming fresh from Western Australia, they can scarcely feel to be impressed by the widely different results of the goldfield discoveries in the two countries" (Editorial, The West Australian, 25.7.1901, p. 4).

Am nächsten Tag, als das königliche Paar Perth verliess, versicherte der Leitartikel ihnen, "To say that Australians have always been loyal to the throne is to repeat what has become a truism ... It had almost seemed as if Australians, being separated from their Sovereign by half the circuit of the globe, were emulous to show that no distance could adversely affect their devotion to the monarchy, as the symbol of the one thoroughly representative institution of the Empire" (Editorial, The West Australian, 26.7.1901, p. 4)

Es gab eindeutig Unterschiede zwischen den Morgenzeitungen der Landeshauptstädte, die im West Australian selbst abzulesen waren. Artikel, die ursprünglich für den Sydney Morning Herald oder vom Argus geschrieben waren, zeigten eine grössere Neigung, Sachverhalte nicht nur landesweit sondern gesamt-australisch zu sehen. Ein Nachdruck aus dem Argus war der Bericht über einen aus 
dem Burenkrieg verwundet zurückgekehrten Soldaten ins ländliche Victoria - ein Artikel, der die Lage in Südafrika und die Auswirkungen auf die dort kämpfenden australischen Soldaten sehr viel deutlicher darstellen konnte, als die zensierten Kurzmeldungen, die ihren Weg über London nahmen. Der nachfolgende Auszug eines zuerst im Sydney Morning Herald erschienenen Artikels widmet sich nach einer amüsanten Einführung dem ernsteren Thema der Bundesarmee und ihrer Kosten:

"Various well-meaning people have flooded the Press with suggestions that all we need to do is to give the men a rifle each and a few packets of ammunition, and encourage them to shoot promiscuously about the country until they learn to judge distance well and to aim accurately. After a few weeks of this sort of practice the men are expected to be a serviceable force of 'self-trained experts', ready to take the field at a moment's notice and to fight when required" ("Our Federal Army and Its Cost", The West Australian, 25.7.1901, p. 2). Der Schreiber dieses Artikels war A.B. [Banjo] Paterson.

Nicht nur die neue australische Armee, auch der australische Journalismus wurde unter die Lupe genommen. Der West Australian widmete der Rede des früheren Leiter des Auslandsabteilung der Londonder Times, die er in Adelaide gab, eine ganzseitige Spalte. Sir Donald Wallace hatte "expressions of great national self-assertiveness which was far more above mere local interest" bemerkt und folgerte daraus, "It was supposed that Australian journalists concerned themselves exclusively with parochial affairs. They had to reclaim a vast wilderness and plant a new civilization in a new country and that, engaged with that gigantic task, they had no time to devote to diplomatic questions... That period, he thought, was now at an end, and the importance of the Australian Commonwealth as a nation would receive due recognition" ("Australian Journalism", The West Australian, 26.7.1901, p. 7).

Zwischen den Komplimenten stand der Aufruf an den australischen Journalismus, seinen Part über das Lokale hinaus auf der Bühne des britischen Empires zu spielen. Die australische Presse war, wie Sir Donald ebenfalls richtig bemerkte, durch und durch britisch geprägt. Der West Australian und sein Herausgeber-cum-Chefredakteur Hackett waren hierbei keine Ausnahme. Seine Vorgaben für die Redaktionsstruktur wie auch die Aufmache der Zeitung bezog er aus Grossbritannien und diese wurden durch Besuche wie der der "Imperial Press Conference" in London 1909 bestärkt (Hunt 1983:151). Um die Jahrhundertwende hatte die Zeitung neben dem Chefredakteur einen stellvertetenden Chefredakteur und Leitartikelschreiber und einen 'chief sub-editor'. Um 1905 wurde ein 'chief-of-staff' eingestellt (Richards 10.4.2001). Die bei britischen Zeitungen übliche dreiteilige Redaktionsführung von Chefredakteur [editor], 'chief sub-editor' und 'chief-of-staff' war somit vollständig und wurde auch in dieser Form bis nach dem Zweiten Weltkrieg beibehalten, auch wenn die Zeitung, ihre Auflage und die Zahl ihrer Angestellten stetig wuchs (Josephi 2000:112/3).

Die Verbindung zum Rest der Welt hatte sich für Perth mit der Eröffnung des Hafens in Fremantle sehr verbessert. 1900 war Fremantle - und nicht mehr Albany - der erste Hafen, der von aus Europa kommenden Schiffen angesteuert wurde (Crowley/de Garis 1969:46). 1889 hatte die Eastern Extension Company ein Kabel zwischen Java und Broome gelegt und 1901 wurde eine weitere Verbindung von Perth über Südafrika nach London fertiggestellt. Trotz dieser Verbindung kamen Nachrichten vom Burenkrieg nie direkt aus Südafrika, es sei denn, sie wurden auf dem Post-, d.h. Seeweg von einem 'Sonderkorrespondenten' geschickt. Berichte wie über den schwer verwundet zurückgekehrten Soldaten im Argus waren daher selten.

\section{4/1918}

1914 waren die Redaktionsräume des West Australian noch immer in den 'West Australian Chambers' in St. Georges Terrace, wo sie seit 1895 untergebracht waren. Das Gebäude hatte nicht, wie später üblich, einen grossen Redaktionsraum. Vielmehr bekamen die Journalisten nach ihrer Volontärszeit ihr eigenes Büro (Richards 1989:2). Die Zeitung war - noch immer mit Sir Winthrop 
Hackett als Herausgeber - von 8-10 auf 12-16 Seiten gewachsen. Die Agenturnachrichten kamen inzwischen aus aller Welt, aber Abbildungen waren noch selten.

Am 4. August 1914 hatte das britische Königreich Deutschland nach seinem Einmarsch in Belgien das Ultimatum gestellt, dessen Neutralität zu respektieren, was einer Kriegserklärung gleichkam.

Während die ersten vier Seiten wie üblich Kleinanzeigen und die gewohnten Spalten enthielten, waren die Seiten 6, 7, 8 und 9 den Ereignissen in Europa gewidmet, wobei auf der oberen Hälfte von Seite 8 eine Karte Europas mit den wichtigsten Strassen- und Eisenbahnverbindungslinien abgedruckt war. Der Leitartikel bedachte Australien mit keinem Wort. Wegen der Zeitversetztheit war nur folgendes bekannt: "The British Empire, so far as known at the time of writing ... is at peace: but who among the million citizens of the Empire dares at this stage to hope that peace will be maintained?" ("Outlook of War", The West Australian, 4.8.1914, p. 8). Der Leitartikel beschäftigte sich erstaunlicherweise fast ausschliesslich mit der Herkunft und den Transportwegen der britischen Nahrungsgüterversorgung, ein Thema, das am nächsten Tag in weiteren, detaillierten Artikeln aufgegriffen wurde ("How Britain is Fed", The West Australian, 5.8.1914, p. 8). Diese Überlegungen, die auch Güter, die aus Australien kamen, miteinbezogen, liessen den West Australian erstens schliessen, dass "whatever the issues may be on land, the German navy must be destroyed," und zweitens dass, "England's moral obligations may urge her into war; her national necessities impel her" ("The Outlook of War", The West Australian, 4.8.1914, p. 8).

So sehr auch die Kommunikationsverbindungen sich verbessert hatten, noch war es schwierig, präzise Nachrichten aus London zu erhalten. "Whenever two friends met, the first question was invariably an inquiry for the latest news of the European situation", aber "there was very little ... that anyone could say with certainty." Interessanterweise fährt der Artikel fort, "Beyond this eager questioning and a natural and patriotic desire to learn of Great Britain's attitude, the excitement did not extend" ("Awaiting the News", The West Australian, 4.8.1914, p. 7). Direkt unter dieser Nachricht wurde ein kurzer Auszug einer Rede Sir John Forrests, inzwischen Bundesschatzmeister, berichtet, der keinen Zweifel darüber zuliess, wie Australien sich im Falle eines Krieges verhalten würde. "If the motherland was in danger, so was the Commonwealth. If Great Britain went to her Armageddon we, as Britishers, would go with her" ("The Dominions and the Mother Country speech by Sir John Forrest, The West Australian, 4.8.1914, p. 7).

Am 5. August 1914 war der gesamte Leitartikel - unter der Überschrift "The War" - der Interpretation der Lage in Europa gewidmet. Auf der in der Mitte der Zeitung als wichtigste Seite dem Leitartikel gegenüberliegenden 'cable page' - der Seite mit den Agenturmeldungen - wurden "Expressions of Loyalty" aus den anderen australischen Landeshauptstädten wie auch Kanada aufgeführt. Für Westaustralien wurde die Rede des Gouverneurs zitiert, der sich ganz anders als Sir John Forrest fasste. Seine Exzellenz, Sir Harry Barron, sagte "Should we go to war, it will affect all of us, and I can only say this: That I feel confident that we, as Australians, will stick together ourselves and also to the British Empire" ("Speech by the Governor", The West Australian, 5.8.1914, p. 7). Der Gouverneur, der wie alle Gouverneure zu dieser Zeit Brite war, dachte eindeutig zuerst an Australien und die noch ungeprüfte Nation, ehe er seine Gedanken dem Empire zuwandte.

1918 erschien die Zeitung noch immer in der gleichen Aufmache und mit gleicher Seitenzahl. Nach Hacketts Tod 1916 war Alfred Langler der Herausgeber und Chefredakteur. Auch wenn die Agenturmeldungen mit den verschiedensten Datumszeilen versehen waren, waren sie durch die Kriegszensur beschränkt. Am 3 September 1918 starb Sir John Forrest auf der Reise nach England vor der Küste von Sierra Leone. Sir John, in Bunbury, im Südwesten Westaustraliens geboren, war auf dem Weg nach England, um seinen Adelstitel und Sitz im Oberhaus einzunehmen. Die Nachricht des Todes erreichte Perth jedoch erst drei Tage später, am 6 September 1918. Am 3. September wurde im Leitartikel eines anderen Todes gedacht - dem von Lenin ("The Death of Lenin", The West Australian, 3.9.1918, p. 7). Lenin, den man einem Attentat zum Opfer gefallen glaubte, starb am 21 Januar 1924. Der Leitartikel zeigt jedoch, wie schwer es noch war, Meldungen zu verifizieren. 
Das wurde zwei Monate später besonders deutlich, als die Nachrichten von den Waffenstillstandsverhandlungen und deren Unterzeichnung nur langsam und stückweise durchdrangen. Die Zeitung fügte jeweils hinzu, welche Nachricht bisher unbestätigt war und zitierte am 9. November das US State Department "that the official news ... may be expected shortly" ("The News Unconfirmed", The West Australian, 9.11.1918, p. 7).

Auf der gleichen Seite wurde unter der Überschrift "Western Australia", die 440. Verwundeten- und Gefallenen-Liste veröffentlicht. Der Leitartikel ging an diesem Tag seltsamerweise nicht auf die Nachrichten des Tages ein, sondern bemühte sich um einen Exkurs in die englische und französische Literatur zu Thackeray, Dumas, Flaubert and Baudelaire.

Am 11. November kannte man die von den Alliierten zum Waffenstillstand gestellten Bedingungen. Diesmal setzte sich der Leitartikel mit den neuesten Nachrichten auseinander - allerdings nicht mit Augenmerk auf ein siegreiches Grossbritannien, sondern auf das Deutsche Reich, das, noch keine fünfzig Jahre alt, vor dem Zusammen-bruch stand. In seinem letzten Satz forderte der Artikel von den Alliierten, dass sie "for a time at least [will have to be] dictators in the country with which they were at war to save it from anarchy" ("Peace or Ruin", The West Australian, 11.11.1918, p. 4). Kein Wort über Australien und die Opfer, die es erbracht hatte.

Die Freude über "the Surrender of the Arch-Enemy" kam am nächsten Tag, als die Friedensnachricht über Amerika zuerst in Melbourne eintraf. Als sie dort vor dem Zeitungsgebäude ausgehängt wurde "thousands raised enthusiastic cheers and sang the National Anthem [God Save the King]." Die Nachricht verbreitete sich in Windeseile, aber der Bericht mahnte zur Vorsicht. "Although the news from America was believed, the populace hoped for a word from Mr. Watt" ("Unprecedented Scenes", The West Australian, 12.11.1918, p. 5).

Der Leitartikel beschäftigte sich ausschliesslich mit der deutschen Kapitulation. Die zweite Ausgabe berichte von "Local Rejoicing" und einem "Festival of Cheering": "And what an inexhaustible fund of subjects there was to found the cheers upon! There were cheers for the Empire, cheers for her Allies, cheers for the various arms of the fighting forces, cheers for the great men of the war, cheers for the soldiers of Australia and of her sister dominions, cheers for those who had gone from Western Australia, cheers for those among them who had gained the V.C., cheers for sick and the wounded, cheers for the returned and cheers, too, for the glorious dead" ("Local Rejoicing", The West Australian, 12.11.1918, p. 5).

\section{7}

Nahezu ein Jahrzehnt später wurde 1927 das Parlamentsgebäude in Canberra eröffnet. Es war auch das Jahr, in dem Sir Alfred Langler starb. H.J. Lambert, der seit 1920 die Leitartikel schrieb, fungierte seit 1926 als Chefredakteur, eine Position, die er bis 1946 bekleidete. The West Australian betrug im Durchschnitt 16 Seiten und enthielt sehr viel mehr Grafiken, besonders in den Anzeigen. Den Nachrichten aus Canberra und den anderen Landeshauptstädten wurde mehr Platz eingeräumt und unter den Weltnachrichten, auch wenn sie aus allen Kontinenten kamen, dominierten die Berichte aus England.

Der Leitartikel an dem eher zufällig ausgewählten Datum vom 12. Oktober 1927 berührte direkt die Frage 'Empire oder Nation?'. Er nahm zum Besuch des britischen Secretary of State for the Dominions, Mr. Amery, Stellung und erwähnte besonders das Ansteigen politischer Reisetätigkeit. Der britische Prime Minister, Mr. Baldwin, hatte vor kurzem Kanada besucht, und nun durfte Westaustralien Mr. Amery willkommen heissen, "who is making a round of business calls on the great self-governing nations, whose affairs, in their Imperial aspects, are his particular concern." Westaustraliens wichtigster Gesprächspunkt beim Ministerbesuch war Grossbritanniens fortlaufende Unterstuitzung der Emigration nach Australien. "Even if the most liberal allowance is made for its uninviting tropics and its arid tracts, Australia has room for a population not less than that of Great Britain" ("Mr. Amery's Visit", The West Australian, 12.10.1927, p. 8) 
Der Leitartikel, der die australische Nation mit grösserer Selbstverständlichkeit als je zuvor erwähnt, griff keine umstrittenen Themen auf. Der sich direkt anschliessende Kommentar geht jedoch entschuldigend auf eine Kontroverse ein. "The controversy over the British historical account of the Gallipoli landing, which has raged in England and Australia over the past few days, has been in the last degree unedifying" ("Current Comment", The West Australian, 12.10.1927, p. 8). Dank später gewonnener Einsichten erscheint die Verteidigung der britischen Geschichtsdarstellung durch den West Australian deplaziert. Der Kommentar zeigt aber, auf welche Seite sich die Zeitung stellte.

\section{3}

Am 7.4.1933, dem Vortag des westaustralischen Sezessionsvotum, widmete der West Australian der Diskussion mehrere Seiten. Noch mehr als bei der Föderation hatte der West Australian "very much a milk and water approach ... Lambert, the editor, was against secession. But on the Board of Directors was Sir Walter James, the former Premier and former Agent General, [who] was all for secession. So Lambert was put under pressure to approve secession, but he made it known that he would resign if the Board insisted on his being for it. So the Board let him have his say, but in a very muted way" (Richards 12.2.2001).

Das Sezessionsreferendum fiel mit der Landtagwahl 1933 zusammen und fand vor dem Hintergrund der hohen Arbeitslosigkeit und wirtschaftlichen Notlage der 'Great Depression' statt. Chefredakteur Lambert räumte den Leserbriefen viel Platz ein, wie auch Berichten über Veranstaltungen für und gegen die Sezession. Gegen die Trennung argumentierte der frühere Kriegspremier Billy Hughes, der vor Geschäftsleute sprach und dabei, der Hörerschaft entsprechend, hauptsächlich auf wirtschaftliche Gesichtspunkte einging. Aber er spielte auch ein paar emotionelle Saiten an. "If secession was good for Western Australia, it could be applied all round, and the British Empire would break up ...After referring in glowing terms to the manner in which Australia had surmounted its war and post-war problems, he exhorted the audience to stand for Australia, united and indivisible" ("Stand for Australia", The West Australian, 7.4.1933, p. 18).

Der Leitartikel räumte ein, dass die Verfassung geändert werden müsse, um nicht die einwohnermässig kleineren Staaten wie Westaustralien zu benachteiligen. Aber er rief nicht dazu auf, für die Sezession zu stimmen, so wie die Zeitung sich vor 33 Jahren für die Föderation ausgesprochen hatte. Auch sprach der Leitartikel nie von Australien als 'unserer Nation', wie Billy Hughes es in seiner Rede getan hatte. Eine solche Bezeichnung hätte im Westaustralien von 1933 den falschen Ton getroffen. Ein anderer Artikel berichtete von der "pro-secession Dominion League rally". Der Redner griff Mr. Hughes Ansprache auf. "Mr. Hughes has described as disloyalists those who were striving for secession, but he had no Union Jack at his meeting in Fremantle, nor had the meeting been opened with the singing of the National Anthem - features which had been observed at the present meeting" ("Secession Rally at Fremantle", The West Australian, 7.4.1933, p. 16).

Das Referendum erbrachte eine grosse Mehrheit für die Sezession - $138.653 \mathrm{zu} 70.706$ (Crowley 1960:274). Allerdings wurde gleichzeitig der Ministerpräsident, der sich für die Trennung eingesetzt hatte, Sir James Mitchell, abgewählt. Wie in den meisten australischen Ländern während der 'Great Depression' gewann die Labor Partei die Wahl. Als die Ergebnisse am Montag, den 10 April 1933 in der Zeitung veröffentlicht wurden, beschäftigte sich der Leitartikel fast ausschliesslich mit der Niederlage der Regierung. Lediglich der letzte Absatz erwähnte das Sezessionsvotum und bemerkte kühl, dass "the vote in favour of secession had gone very much as was expected" und schloss, "It may be assumed, therefore, that the next step will be to present the decision to the Imperial authorities, with reasons in justification of the dissatisfaction which has been so emphatically expressed" ("Defeat of the Government", The West Australian, 10.4.1933, p. 12).

Knappe 33 Jahre nach der Zusammenschluss zum Commonwealth of Australia war Westaustralien ungleich weiter von einem in der australischen Nation verkörperten Gemeinschaftsgefühl entfernt, als zu dem Zeitpunkt, als es für den Bund votierte. 


\section{Fazit}

In den 33 Jahren, die hier untersucht wurden, gab es keine Zeitspanne, von der man sagen kann, dass der West Australian in seiner Einstellung die Schwelle von einem britisch-imperialen zu einem australisch-nationalen Bewusstsein überschritten hatte. Am ehesten trifft die Beschreibung westaustralisch-imperial für die Bewusstseinslage $\mathrm{zu}$, denn der West Australian fühlte sich verständlicherweise in allererster Linie Westaustralien verbunden. In zweiter Linie sah er sich dem Empire verpflichtet, während die Zugehörigkeit zur australischen Nation 1933 einen weit abgeschlagenen dritten Platz einnahm. Wie Kelly hervorhob, war Australien "a child of the Empire". Es war keine Nation, die sich in Opposition zum Reich gebildet hatte - einem Entwicklungsmuster, auf dem Andersons Theorie des Entstehens eines Nationalbewusstseins basiert.

Nimmt man das Anderson'sche Konzept einer Nation, muss man folgern, dass die Leitartikel des West Australian in der untersuchten Zeitspanne in ihrem Diskurs keine Nation konstruieren. Selbst wenn man Kellys Vorschlag der Verträglichkeit von Empire und Nation folgt, kommt man zum Schluss, dass der West Australian nur mit sehr zögerlichen Schritten auf die Nation zugeht, der es rechtlich angehört. Die Zeitung versuchte nicht, die Idee der australischen Nation mit emotionellen oder geistigen Gütern zu befrachten. Im Bundesland mit dem höchsten Anteil britischer Immigranten war das Empfinden, von den Ostküste-Staaten nicht gerecht behandelt zu werden, oder um einen australischen Ausdruck zu gebrauchen - 'not being given a fair go' - eine zu grosse Herausforderung für das sich gerade erst entwickelnde Zusammengehörigkeitsgefühl - vor allem in Zeiten der wirtschaftlichen Not.

Was die Redaktionsstruktur der Zeitung angeht, so hatte die Föderation kaum Einfluss auf die Zeitung. Die 'cable page' mit den Auslandsnachrichten brachte, zuerst aus Notwendigkeit, später aus freier Wahl, hauptsächlich britische Nachrichten, was auch den familiären und finanziellen Verbindungen zu Grossbritannien entsprach. Technische Entwicklungen machten es erst nach dem Zweiten Weltkrieg möglich, dass sich der West Australian den gesamten Bundesstaat als Vertriebsgebiet erschloss, wie sich auch erst nach dem Zweiten Weltkrieg mit dem Kriegsschauplatz im Pazifik, der direkten Bedrohung Australiens und den darauf folgenden Immigrationwellen, die die anglo-irische Prädominanz unterhöhlten, ein für alle Mal die Waagschale zugunsten der australischen Nation neigte.

\section{Sekundärliteratur}

Anderson, Benedict, 1983/1991. Imagined communities, New York: Verso.

Blainey, Geoffrey, 1966. The tyranny of distance, Melbourne: Sun Books.

Crowley, K.F., 1960. Australia's western third, London: Macmillan.

Crowley, K.F., Brian de Garis, 1969. A short history of Western Australia, Melbourne: Macmillan.

Cryle, Denis, ed., 1997. Disreputable profession - journalists and journalism in colonial Australia, Rockhampton: Central Queensland University Press.

Cryle, Denis, 1996. Colonial journalist and journalism: an overview, in: Denis Cryle, ed., 1997, Disreputable profession - journalists and journalism in colonial Australia, Rockhampton: Central Queensland University Press, 1-19.

Curthoys, Ann, Julianne Schultz, ed., 1999. journalism - print, politics and popular culture, Brisbane: University of Queensland Press.

Gaylard, G., A. Savvas, 1993. One hundred \& sixty years of news from The West Australian. Frewville, S.A.: Adrian Savvas.

Hackett, General Sir John, 1997. Obituary. The Times. 10.9.1997, p. 21.

Harcourt, Edgar, 1987. Taming the tyrant - The first 100 years of Australia's international telecommunication services, Sydney: Allen \& Unwin. 
Headrick, Daniel R., 1981. The tools of empire - technology and European imperialism in the nineteenth century, Oxford: Oxford University Press.

Hunt, Lyall, 1983. Hackett, Sir John Winthrop, in: Bede Nairn, Geoffrey Serle, eds, 1983. Australian dictionary of biography, vol. 9, 1891-1939, Melbourne: Melbourne Unversity Press, 150-153.

Josephi, Beate, 2000. A matter of organisation, Australian Studies in Journalism 9, 106-125.

Kelly, Paul, 2001. 100 years - The Australian story, Sydney: Allen \& Unwin.

Kirkpatrick, Rod, 2000. Country conscience - a history of the NSW provincial press 1841-1995, Canberra: Infinite Harvest Publishing.

Lawson, Sylvia, 1999. Print Circus: The Bulletin from 1880 to federation, in: Ann Curthoys, Julianne Schultz, eds, 1999. Journalism - print, politics and popular Culture, Brisbane: University of Quensland Press, 83-96.

Lloyd, Clem, 1999. British press traditions, colonial governors, and the struggle for a 'free' press, in: Ann Curthoys, Julianne Schultz, ed, 1999. Journalism - print, politics and popular culture, Brisbane: University of Quensland Press, 10-19.

Mayer, Henry, 1964. The press in Australia, Melbourne: Lansdowne Press.

Moyal, Ann, 1984. Clear across Australia - a history of telecommunications, Melbourne: Nelson Publishers.

Nairn, Bede, Geoffrey Serle, eds, 1983. Australian dictionary of biography, vol. 9, 1891-1939, Melbourne: Melbourne Unversity Press.

Porter, Ann, 1983. Langler, Sir Alfred, in: Bede Nairn, Geoffrey Serle, eds, 1983, Australian dictionary of biography, vol. 9, 1891-1939, Melbourne: Melbourne Unversity Press, 667-668.

Putnis, Peter, 1999. The press cable monopoly 1895-1909 - a case study of Australian media policy development, Media International Australia 90, 139-155.

Rantanen, Terhi, 1997. The globalization of electronic news in the 19th century, Media, Culture and Society 19, 605-620.

Richards, W.T.G. (Griff), 2001. Personal interviews, The West Australian, 12 February 2001, 10 April 2001. [editor of The West Australian 1956 - 1972]

Richards, W.T.G., 1989. A fitting symbol of a proud past, The West Australian, Liftout, 29.9.1989, pp $2 / 3$.

Stannage, C.T., ed., 1981. A new history of Western Australia, Perth: University of Western Australia Press.

West Australian, The, 1979. 100 Years, The West Australian, 17.11.1979, pp 39-42. 


\section{Fünfzig Jahre Anstrengung für hundert Jahre Föderation}

\section{Gerd Leutenecker, Wehr}

Am 1. Januar 2001 versammelten sich zigtausend Menschen in Australien, um das Einhundertjährige Bestehen des Bundesstaates Australien zu feiern. Hundert Jahre zuvor war es ein ähnlich imposantes Feiern, als der Neubeginn Australiens gefeiert wurde. Sechs unabhängige Kolonien schlossen sich zu einem "föderalen Staatenbund" zusammen. Die Arbeit von Jahrzehnten fand einen Schlusspunkt der gleichzeitig der Startpunkt für die gemeinsame Zukunft werden sollte.

Die Australier haben aber lange Jahre ihre Gründungsväter vergessen. Canberra ist das Symbol für den Australischen Staatenbund. Wenn man sich in der Bundeshauptstadt Canberra umschaut, so fällt einem auf, dass die einzelnen Stadtteile zwar auch Namen von berühmten australischen Staatsmänner haben, diese aber im Grunde politische Personen des 20. Jahrhunderts waren, indem sie Staatsämter inne hatten. Weitgehend gleich verhält es sich bei der Namensgebung der australischen Wahlkreise. Der größte Wahlkreis in New South Wales, der sich von Broken Hill bis Dubbo und hin zur Grenze mit Queensland erstreckt, trägt den Namen Parkes. Damit ist zumindest im Outback Henry Parkes, landläufig als der Gründungsvater der Föderation bezeichnet, verewigt.

Australien hat sich vornehmlich auf das 20. Jahrhundert konzentriert. Ihr Jahrhundert, das die identitätsstiftenden Momente für das australische Verständnis liefert. Als wäre keine Vorgeschichte gewesen, schwankte die Aufarbeitung zwischen dem Scham der Sträflingskolonien und dem antiquiertem britischen Untertan. Die eigene kulturelle, gesellschaftliche und politische Historie ist vielleicht zeitlich zu nahe gewesen, das man sich dieser vergewissert hätte. Eine Parallele zur amerikanischen Glorifizierung der staatlichen Gründungsphase ist in Australien nicht auszumachen. Auch heute, anlässlich der Einhundertjahrfeiern Commonwealth of Australia ist das Hauptaugenmerk auf die australischen Errungenschaften des 20. Jahrhunderts gerichtet. Es gab dennoch in den Tageszeitungen Sydney Morning Herald und The Age eine lose Folge von Artikeln namhafter Wissenschaftlern zur Vorgeschichte der Föderation; daran ist feststellbar, dass eine Erinnerung an diese bedeutende historische Errungenschaft mehr den Charakter der wissensvermittelnden Funktion inne hat.

Politische Union, Zollunion, Konföderation, Staatenverbund und Staatenbund - in mehr als fünfzig Jahren vor der offiziellen Staatengründung Australiens 1901 sind diese Ziele formuliert, angestrebt aber auch wieder verworfen worden. Was war Australien vor der Abtrennung von Victoria (1851) und Queensland (1859) von der "Mutterkolonie"? Ein nahezu geeinter Kontinent im Verständnis von New South Wales als das Synonym für Australien. Mit weitgehenden politischen Rechte, ausgestattet von Großbritannien, und einer aufstrebenden gesellschaftlichen Elite, die die eigenen Angelegenheiten vor Ort regeln wollte und konnte. Eben zu diesem Zeitpunkt ist der erste Gedanke nachweisbar, der sich Australien als politisches Ganzes konkret vorstellte. 1847 unternahm Earl Grey, der dann aktuelle britische Colonial Secretary, den Versuch und schlug eine General Assembly vor, mit einer wünschenswerten Zentralmacht für Australien als ganzes. Australien war dabei das Synonym für Australasia, also nicht das Festland alleine, sondern mit Tasmanien (Van Diemens Land), Neuseeland und den weiteren kleineren Inseln im Südpazifik, die unter britischem Einfluss standen. Dieser Vorstoß blieb ohne Widerhall, da die zuständigen kolonialen Stellen nicht mit einbezogen waren oder gar für die Ausgestaltung der Idee konsultiert wurden. Die Kolonien wurden im puren britischen Verständnis behandelt. Die Reaktionen darauf waren die emotionale und politische Ablehnung. 1847 war der falsche Zeitpunkt gepaart mit der unzeitgemäßen Vorgehensweise von Seiten des Mutterlandes für eine "federal authority", die von Teilen in Australien bereits gewünscht wurde. Die entwicklungsstrukturell intendierte Abspaltung Victorias und Queensland von N.S.W. kam dennoch hinzu, und damit verschlechterten sich auch die allgemeinen Handelsbedingungen untereinander. Sicherlich, nicht jeder Handel treibende oder 
Politiker sah dies zu dieser Zeit ebenfalls so, aber im Outback wurde man an den Grenzen de fakto zum Fremden, wenn man die Koloniengrenzen überschritt.

Der nächste Schritt war erneut ein reagieren, weniger ein agieren auf zunehmende Probleme im Inneren. Die Binnenwirtschaft hatte ihre Grenzen. Dabei lässt sich eine von Teilen gewünschte Zollunion ausmachen. Erste Vorstöße gehen bis in die 1860er zurück. Diese "commercial federation" sollte nur als Hilfskonstrukt dienen und keine echte staatliche Integration ermöglichen. Diese hauptsächlich ökonomische ordnungspolitische Grundkonstellation aber ist in der zweiten Hälfte des 19. Jahrhunderts nicht zu unterschätzen. Das Miteinander wirtschaften war nicht eine Wunschvorstellung weniger sondern entsprach der Notwendigkeit auf dem australischen Kontinent. Die regelmäßig stattfindenden "intercolonial conferences" der Premiers, hatten natürlich auch die Koordinierung gemeinsamer Politikfelder zum Inhalt. Man traf sich zwischen 1860 und 1900 im Durchschnitt zwei mal pro Jahr. Hier wurden bereits die gegensätzlichen Auffassungen aber auch die gemeinsamen australischen Notwendigkeiten debattiert. Die Grenzen des uniert Machbaren in den vorgegebenen Rahmen traten erkennbar zu Tage. Von Quarantänebestimmungen, gemeinsame Kommunikationswege (Telegraphenleitung) bis hin $\mathrm{zu}$ problematischsten Kontroversen der Handelsbestimmungen, die Themen für den Föderalstaat Australien waren vorgegeben. Im weitläufigen Areal der Riverina (geographisch, nicht der heutige politisch determinierte kleinere Teil) mit ihren Flüssen und der Zugverbindung, traten die Probleme und Rivalitäten des grenzüberschreitenden Handels in vielfältiger Weise zu Tage.

Ein Fakt aber ist, dass bis in die 80er Jahre des 19. Jahrhunderts alle australische Kolonien vorrangig daran interessiert waren, ihre eigene staatlichen Institutionen und die in vorgegebenen Grenzen souveräne Regierungsfähigkeit auszubauen. Diese eigenständige Orientierung lies nur wenig Platz in der Tagespolitik für den Gedanken und das Handeln für die Föderation.

Gesellschaftlich sind in diesem Zeitraum zwei gegenläufig auszumachende Phänomene feststellbar: Einerseits die starke Identifikation mit der eigenen Kolonie und andererseits in gewisser Weise auch die gekeimte gemeinsame australische Identität. Die ersten Generationen von in Australien geborenen etablierten sich. Zwischen Koloniennationalismus und britischem Subjekt reifte das 'mateship'. Hier findet man auch den nächsten Schritt zur Föderation: die Australian Natives' Association. 1871 gegründet entsprach sie dem Zeitgeist. Nur Männer, die in Australien geboren waren, konnten Mitglieder werden. Allgemein wird sie als eine Verbindung der Arbeitnehmerschaft charakterisiert, die das Anliegen der Föderation propagierte. Ihre Struktur war bereits über die Koloniengrenzen hinweg angelegt, so konnte sie sich an der gesellschaftlichen Verankerung des Ziels einer Nation intensiv beteiligen. Die Australian Natives' Association propagierte den Bundesstaat. Die Materie kam raus aus den Parlamenten und hin zur populistischen Bewegung.

Wieder im politischen System gründete sich der Federal Council of Australasia. Dies ist das erste staatlich institutionalisierte Bindeglied, das das Ziel einer australischen Union erörterte. Das Ziel war eine australische Stimme in Großbritannien zu haben, um ein größeres Augenmerk auf die regionale bzw. geostrategischen Belange zu erzeugen. Die Legislativmacht über eine gemeinsame Außenpolitik war dem Gesetz her da. Aber, da die Initiative von Victoria ausging, nahmen New South Wales und South Australia daran nicht teil. In der Person des Premiers von N.S.W., Henry Parkes, sind die Gründe dafür zu suchen. Er strebte bereits zu diesem Zeitpunkt eine bundesstaatliche Lösung mit legislativer und exekutiver Kompetenz an. Im Grunde genommen war mit dem Federal Council of Australasia von britischer Seite her eine Instanz geschaffen, die eine Legislativmacht über die Angelegenheiten Australiens hatte, aber immer unter der strikten Einbeziehung der einzelnen Kolonialparlamente! Dieses staatlich-institutionelles Ungetüm rekrutierte sich aus den Kolonialexekutiven, nahm hoheitliche Legislativrechte in Anspruch und sollte die eigenen getroffenen Entscheidungen dann wiederum in der eigenen Kolonienlegislative absegnen lassen. Ein anachronistisches System, das aber zumindest ein Versuch wert war.

Außenpolitik hieß Verteidigungspolitik. Und eine gemeinsame Verteidigungspolitik blieb die Klammer für das Zusammenwachsen. Nach 1888, als General Edwards für das britische Colonial 
Office seinen Lagebericht zur Verteidigungsfähigkeit und den notwendigen australischen Anstrengungen verfasst hatte, begann eine neue Runde hin zum Föderalstaat. Henry Parks nahm in seiner berühmten Tenterfield-Rede die Argumente von General Edwards auf und führte dies hin zur Notwendigkeit einer neuen "intercolonial convention" und auf dem Wege der Ausarbeitung einer australischen Verfassung das Ziel der Föderation zu erreichen.

Die 1890er sind der Wendepunkt in der australischen Föderalismusgeschichte.

Die Australasian Federation Conference traf sich am 6. Februar 1890 in Melbourne, mit dem festen Vorsatz, die Einigung Australiens zu verwirklichen. Der vielzitierte Ausdruck an diesem ersten Tag, summierte die Hauptprobleme: Der "Lion in the Path" stammt von James Service, Premier von Victoria. Er hat es am Eröffnungsbanquet in seiner Rede ausführlich ausgeführt, dass die interkolonialen Zölle noch immer die Föderation zur Strecke bringen könne, daher müsse zuerst der Löwe zur Strecke gebracht werden. Es folgte vom 2.März bis zum 9.April 1891 in Sydney die Ausarbeitung der (ersten) Verfassung für den Bundesstaat Australien. Der Löwe war gebändigt, denn im zukünftig föderal organisierten Australien werde es keine internen Handelszölle mehr geben. Die Delegierten gingen mit diesen Ergebnissen zurück in ihre Kolonien, und sollten die Verfassung in ihren jeweiligen Parlamenten zur Abstimmung bringen. Das Morgenlicht des Bundesstaates Australien schimmerte, kam aber gegen den Nebel noch nicht an. Die Parlamente befassten sich nicht mit der Verfassung, es standen teilweise Neuwahlen an, Regierungswechsel folgten, politische Themenverschiebungen setzten ein und die allgemeinen politischen Rahmenbedingungen änderten sich; eine schwere Depression überzog die australischen Kolonien, mit einziger Ausnahme von Western Australia, das durch seine neuen Goldfeldern wirtschaftlich aufblühte. Dringend notwendige soziale Reformen waren die unaufschiebbaren Themen in den Parlamenten, nicht die Föderation.

Mit den staatlichen Instanzen war zu diesem Moment kein glückliches Gelingen möglich. Abermals setzte eine populistische Bewegung ein: Auf der Corowa Conference vom 31. Juli bis 1. August 1893 trafen sich Vertreter der Australian Natives' Association mit der Federation Leagues, die zu diesem Zeitpunkt die Interessensvertretung für die Handeltreibenden und die Farmer war. Eine eher unscheinbare Resolution von John Quick eingebracht, markierte das weitere Vorgehen. Es müsse im Prozess der Föderation verstärkt auf die Einbeziehung des Volkes wert gelegt werden, die Direktwahl der Delegierten und eine Referendumsentscheidung über die neu auszuarbeitenden Verfassung müsse abgehalten werden bevor sie den Parlamenten vorgelegt werde. Etwas absolut neues also. Der Wähler sollte die legitime Adresse sein und damit die direkte Legitimation der neuen Verfassung vom Volke ausgehen. Revolutionär in anderen Teilen der damaligen Welt. Der neue Premier von N.S.W. George Reid fand Gefallen an dieser strukturellen Vorgehensweise. Auf der Premiers' Konferenz im Hobart 1895 einigte man sich dem Sinn der Resolution nach, auf die neue Struktur.

1895 begann die letzte Runde im Ringen um den Staatenbund Australien. Den Vorstellung von Quick folgend, sollte ein erneuter Anlauf für eine "constitutional convention" gestartet werden. Es lag in den Händen der Premiers, die den legislativen Weg bereiten sollten, dass Delegierte gewählt werden sollten. Western Australia stellte die Ausnahme dar, hier wurden die Delegierten von den Mitgliedern des Parlamentes bestimmt. In South Australia durften Frauen mitwählen, sie hatten bereits zu diesem Zeitpunkt das Wahlrecht. Das Parlament von Queensland entschied sich mehrheitlich gegen eine Teilnahme, indem es die Volkswahl der Delegierten ablehnet! Und Neuseeland beendete die Teilnahme.

Um einem weiteren Debakel entgegenzutreten, folgte 1896 in Bathurst die sog. People's Federal Convention. Im öffentlichen Bewusstsein war der Einigungsgedanke verankert. Es sollte Druck auf die Regierungen ausgeübt werden, dass die neue Constitutional Convention auch zügig organisiert werde, beginnend mit der Wahl der Delegierten, die im März 1897 dann in vier Kolonien stattfand. 
Das erste Treffen fand in 1897 in Adelaide statt. Die Neue Verfassung wurde ausgearbeitet. Diese wurde an die Parlamente weitergeleitet, um für Änderungswünsche eine breite Basis zu erhalten. Es stellt Bemerkenswerterweise eine doppelte Legitimationsbasis dar: Die Entscheidungen lassen sich immer auf das Volk und seinen Willen zurückführen, sei es für die Parlamentarier aber auch den Delegierten. Die praktikabel größtmögliche Beteiligung des Volkes fand in Australien statt. Keine einsame exekutive Endergebnisse wurden dem mündigen Volk präsentiert - die Mehrheit der Bürger wusste um was es geht. Man traf sich dann 1897 und 1898 zur abschließenden verfassungsgebenden Versammlungen in Melbourne und Sydney. Diese wurde im Anschluss daran dem Volk zur Abstimmung gegeben.

Aber das Referendum von 1898 wurde in N.S.W. ein Fehlschlag. Das hoch gesetzte Quorum wurde nicht erreicht, und wenn die größte und älteste Kolonie sich nicht beteiligen würde, wäre das Ziel der Föderation nicht erreicht. Auf dem Premiers Meeting im Januar 1899 in Melbourne an dem auch Queensland teilnahm, wurden kleine Änderungen vorgenommen wie etwa, dass die zukünftige Bundeshauptstadt näher an Sydney sein müsse. In der zweite Referendumsrunde wurde die Verfassung mehrheitlich angenommen, dieses mal nahm auch Queensland teil, aber eine Entscheidung von Western Australia fehlte noch. 1899 erhielten die Frauen das Wahlrecht in W. A.. Politischer Hintergrund war, dass man die Hoffnung auf die Frauen setzte, dass sie mehrheitlich für die Föderation stimmen würden und damit der fälschlicherweise angenommenen eventuell höheren Anzahl von Gegenstimmen von Seiten der Minenarbeiter entgegenzuwirken. Es war im Grunde nicht mehr das Problem der Wahlbürger, ob man sich zum Bundesstaat zusammenschließen solle, sondern ob man diese Verfassung dafür als geeignet betrachtet! Die Frage nach dem letztendlichen Wahlerfolg für das neue Staatenkonstrukt lässt sich zugespitzt in der Art formulieren, dass eine Mehrheit sich dadurch auch positive Effekte versprach, im individuellen Wohlergehen, speziell aber der wirtschaftlichen Besserstellung.

Der letzte Schritt führte notwendigerweise 1900 nach London. Vier australische Delegierte und ein Beobachter (Western Australia) kamen in London an, um ihr Mandat zu erfüllen, dem parlamentarischen und imperial-hoheitlichen Prozedere in Westminster beizuwohnen. Ein herausragendes Problem für London war, dass das Privy Council immer noch letzte Instanz für australische Gerichte sein müsse. Alfred Deakin war es, der gegen Joseph Chamberlain anging. Sein Argument war, dass die britische Regierung genügend Zeit hatte, sich an der Verfassung zu beteiligen (in der Form von "gewünschten" Änderungen), und das australische Volk bereits darüber abgestimmt hatte. Das Verständnis von Mutterland und Kolonie schimmerte noch immer im gegenseitigen Umgang miteinander durch. Chamberlain konnte nicht zurückweichen und nur seine Kompromissbereitschaft dadurch belegen, das die Regularien der Wege zum letztinstanzlichen Privy Council debattiert wurden. Letztendlich mussten die vier Delegierten diesen britischen Entschluss aber akzeptieren. Am 14. Mai wurde der Gesetzesvorschlag in das House of Commons eingebracht. Das "Commonwealth of Australia Bill" wurde im Juli verabschiedet, und Königin Victoria gab am 9. Juli 1900 ihre Zustimmung.

Zurück in Australien ging die Föderation ihren Weg. Der neu eingetroffene erste GeneralGouverneur, Lord Hopetoun, hatte die ihm zugedachte Aufgabe, den ersten Prime Minister zu ernennen. Und in Verkennung der Persönlichkeiten, nominierte er William Lyne, in diesem Moment gerade Premier von N.S.W., indessen gleichzeitig auch ein bekennender Gegner der Föderation. Keiner wollte und konnte mit ihm zusammenarbeiten und er gab selber den Weg frei für Edmund Barton. Die Inaugurationsfeiern konnten mit einem amtierenden und respektierten Prime Minister of Australia am 1. Januar 1901 stattfinden.

Die fünf wesentlichen Strukturmerkmal der australischen Föderation sind demnach: 1. eine geschriebene Verfassung mit doppelter Gewaltenteilung, die durch gewählte Delegierte ausgearbeitet wurde; 2. eine Volksabstimmung, um eine größtmögliche Legitimierung für den Staat zu erhalten; 3. der neue Staat hat durch die Kolonialparlamente die legislative Weihen erfahren, so dass konstatiert werden kann, die Kolonien haben bei der Beschneidung ihrer Rechte mitgewirkt; 4. 
die staatsrechtlich unentbehrliche Anerkennung durch das britische Parlament was sozusagen die zweite Legislativweihung war, und 5. die königliche Billigung.

Der australische Staatenbund ist nicht alleinig als Föderation ohne seine Verfassungsentwicklung erklärbar, so wie die Verfassung nicht alleinig erklärbar ist ohne Föderation. Die Intention sich zusammenzuschließen über den Weg der Föderation mit vertragsrechtlichem Konstrukt war nicht neu, Australien konnte bereits in den 1890er auf fünf Staaten in der Welt blicken, die diesen Weg gegangen sind. Der langatmige Weg hätte nicht ohne den Willen des Volkes so zum Erfolg gebracht werden können, als einzelnen Politikern zeitweilig die Luft ausgegangen ist, und die Bewegung hin zur Föderation vom Volke ausging. Ein durch und durch demokratisch gesinntes Empfinden, trotz mehrheitlich rassistischer Grundhaltung. Ein Makel, der Australien noch lange anhaftete.

Ein Auswahl von weiterführender Literatur ohne Zeitschriftenartikel. Stichworte: Föderation, Staatengründung, Verfassungs"väter", politisch-historische Relevanz.

\section{Zeitgenössische Literatur und Quellen}

Allin, C.D., 1907. Early federation movement in Australia. Kingston, Ontario

Anderson, H., Hsg., 1991. Tocsin: Radical arguments against federation 1897-1900, Melbourne Australasian Federal Convention: Official record of the debates of the Australasian Federal Convention, Sydney 1986 (Adelaide 1897, Sydney 1897, Melbourne 1898).

Australasian Federation League, 1894-95. The Commonwealth, Journal of the Australasian Federation League in NSW.

Australasian Federation League, (April-September) 1898. The Australian federalist, Journal of the Federal Association. [journal established by the Australasian Federal League for the referendum]

Australasian Federation League, 1898. The Federalist, Journal of the Tasmanian branch of the Federal Association.

Australia, 1893. Federation Conference, Official Report of the Federation Conference held in the Court House, Corowa, on Monday 31 st July and Tuesday 1st August 1893, Corowa.

Baker, Sir R, 1897. The executive in a Federation, Adelaide.

Baker, Sir R, 1897. Federation, Adelaide.

Baker, Sir R, 1891. A manual of reference to authorities for the use of the members of the National Australasian Convention, Adelaide.

Bendigo League: The new federation movement: the Corowa Federation Conference, report of the Bendigo delegates, submitted to the Sandhurst branch of the Australian Natives' Association, in: The new federation movement, from the Corowa Conference, 1 August 1893, to the ANA Conference, 27 March 1894, Bendigo.

Clark, A., 1900. The federal financial problem and its solution, Hobart.

Clark, A., 1901. Studies in Australian constitutional law, Melbourne.

Clark, C. M. H., 1955. Select documents in Australian history 1851-1900, Sydney.

Crichton, P., P. Hardy, M. Stell, 1992. Federation bibliography, Canberra.

Dalley, W., 1884. Speeches on the proposed Federal Council of Australasia and the resolutions of the Convention, Sydney.

Deakin, A., 1963. The federal story: The inner history of the federal cause 1880-1900, Carlton.

Deakin, A., 1995. And be one people: Alfred Deakin's federal story, introduced by Stuart Macintyre, Carlton.

Dilke, C., (June) 1891. The Commonwealth of Australia, The Forum 11.

Drake, J., 1896. Federation: Imperial or democratic?, Brisbane 1896.

Fraser, J., 1910. Australia: The making of a nation, London.

Garran, R., 1897. The coming Commonwealth, Sydney. 
Garran, R., 1958. Prosper the Commonwealth, Sydney.

Gay, W., M. Sampson, 1895. The Commonwealth and the Empire, o.O.

Griffith, S., 1899. Australian federation and the draft Commonwealth Bill, Brisbane.

Griffith, S., 1896. Some conditions of Australian federation, Brisbane.

Hughes, W.M., W. Dick, 1897. Federation as proposed by the Adelaide Convention, Adelaide.

James, W., 1949. The James papers: letters on federalism, Australian Quarterly 21(4).

Keenan, J., 1904. The inaugural celebrations of the Commonwealth of Australia, Sydney.

Kingston, C., (February) 1896. The democratic element in Australian federalism, Review of Reviews.

O'Sullivan, E.W., 1900. From colony to Commonwealth, Sydney.

Parkes, H., 1895. Speech on the policy of the Reid Ministry as opposed to federation delivered in the Legislative Assembly, 30 May 1895, Parramatta.

Parkes, H., 1892. Fifty years in the making of Australian history, London.

Parkes, H., 1891. One people, one destiny: Speech of the Hon. Sir Henry Parkes, GCMG, to the Citizens of Sydney, in the Gaiety Theatre, Saturday, 13 June 1891, Sydney.

Parkes, H., 1890. The federal government of Australasia: Speeches delivered on various occasions (November 1888-May 1890), Sydney.

Perkins, E., D. Headon, Hsg., 1997. Our first republicans: Selected writings of John Dunmore Lang, Charles Harpur and Daniel Henry Deniehy 1840-70, Sydney.

Quick, J., R.R. Garran, 1901. The annotated constitution of the Australian Commonwealth, Sydney.

Quick, J., 1965. Sir John Quick's notebook, hsg. v. Fredman, L., Newcastle.

Reid, G., 1917. My reminiscences, London.

Robertson, A., 1897. Federation and afterwards: A fragment of history, Sydney.

Tomm, L., (August) 1897. The referendum in Australia and New Zealand, Contemporary Review.

Wise, B.R., 1913. The making of the Australian Commonwealth 1889-1900, London.

\section{Monographien und Sammelbände}

Alomes, Stephen, 1988. A nation at last?: the changing character of Australian nationalism 18801988, North Ryde.

Anderson, Margaret, 1998. When Australia was a woman: images of a nation, Perth.

Scott, Ernest, hsg., 1988. The Cambridge history of the British Empire. [Australia, vol. 7, 425-453], Cambridge.

Garran, R., 1997. The Federation Movement and the founding of the Commonwealth Australia: The constitution makers. (= Papers on Parliament Nr. 30). Canberra: Department of the Senate.

Australian dictionary of biography, 1966. Carlton.

Aveling, M., 1970. A history of the Australian Natives' Association 1871-1900, Monash.

Bannon, J., 1994. The crucial colony: South Australia's role in reviving federation 1891 to 1897, Canberra.

Bennett, S., Hsg., 1975. Federation, Melbourne.

Bennett, S., 1971. The making of the Commonwealth, Melbourne.

Birrell, Robert, 1995. A nation of our own: citizenship and nation-building in federation, Melbourne.

Bunbury, Bill, 1998. Unfinished business: reconciliation, the republic and the constitution, Sydney.

Chesterman, John, Brian Galligan, 1997. Citizens without rights: Aborigines and Australian citizenship, Cambridge.

Clark, C.M.H., 1981. A history of Australia, vol. 5 (The people make laws, 1888 - 1915), Carlton. 
Craven, G., Hsg., 1992. Australian federation: Towards the second century, Carlton.

Craven, G., 1986. The Convention debates 1891-1898: Commentaries, indices and guide, Sydney.

Crisp, L.F, 1990. Federation fathers. Carlton.

Crowley, F., Hsg., 1974. A new history of Australia, Melbourne.

Cunneen, C., 1983. King's men: Australia's Governors-General from Hopetoun to Isaacs, Sydney.

Davidson, A., 1991. The invisible state: The formation of the Australian state 1788- 1901, Melbourne.

Dermody, K., 1997. A nation at last: The story of federation, Canberra.

Ely, Richard, 1976. Unto God and Caesar: religious issues in the emerging Commonwealth, 1891-1906, Carlton.

Evans, R., C. Moore, K. Saunders, B. Jamison, B., 1997. 1901: Our future's past: Documenting Australia's federation, Sydney.

Fitzhardinge, L., 1964. That fiery particle: William Morris Hughes, a political biography, vol. 1, 1862-1914, Sydney.

Gabay, A., 1992. The mystic life of Alfred Deakin, New York.

Glass, M., 1997. Charles Cameron Kingston, Carlton.

Graham, A.D., 1939. The life of the Right Honourable Sir Samuel Walker Griffith, Brisbane.

Grimshaw, P., M. Lake, A. McGrath, M. Quartly, 1994. Creating a nation, Melbourne.

Greenwood, G., Hsg., 1997. Australia: a social and political history. London.

Haward, M., J. Warden, Hsg., 1995. An Australian democrat: The life, work and consequences of Andrew Inglis Clark, Hobart.

Hirst, J.B., 1998. Discovering democracy: a guide to government and law in Australia, Carlton South.

Hodgins, Bruce W., Don Wright, W.H. Heick, Hsg., 1978. Federalism in Canada and Australia: The early years, Canberra.

Irving, Helen, Hsg., 1999. The Centenary companion to Australian federation, Cambridge, Melbourne.

Irving, Helen, 1997. To constitute a nation: A cultural history of Australia's constitution, Melbourne.

Irving, Helen, 1996. A woman's constitution?: Gender and history in the Australian Commonwealth, Sydney.

La Nauze, J. A., 1972. The making of the Australian constitution, Carlton.

Livingston, K. T., 1996. The wired nation continent: the communication revolution and federating Australia, Melbourne.

Martin, A., 1908. Henry Parkes: A biography, Carlton.

Macintyre, S., 1986. The Oxford history of Australia, vol. 4, The succeeding age: 1901-1942, Melbourne.

Margarey, S., S. Rowley, S. Sheridan, 1993. Debutante nation: Feminism contests the 1890s, Sydney.

McKenna, Mark, 1996. The captive republic: a history of republicanism in Australia 1788-1996, Cambridge.

McMinn, W.G., 1994. Nationalism and federalism in Australia, Melbourne.

McMinn, W.G., 1989. George Reid, Carlton.

McMinn, W.G., 1979. A constitutional history of Australia, Melbourne.

McMullin, R., 1991. The light on the hill: The Australian Labor Party 1891-1991, Melbourne. 
Martin, A.W., Hsg., 1969. Essays in Australian federation, Melbourne.

Matthews, Brian, 1999. Federation, Melbourne.

Norris, Ronald, 1975. The emergent Commonwealth: Australian federation, expectations and fulfilment 1889-1910, Carlton.

O'Collins, G., Patric. McMahon, 1965. A founder of Australian federation, Carlton.

Reynolds, J., 1984. Edmund Barton, Sydney.

Rickard, J., 1976. Class and politics: New South Wales, Victoria and the early Commonwealth 1890-1910, Canberra.

Russell, Roslyn, Philip Chubb, 1998. One destiny!: The federation story, how Australia became a nation, Ringwood.

Saunders, C., 1989. The Constitutional framework: Hybrid, derivative but Australian, Melbourne.

Souter, Gavin, 1976. Lion and kangaroo: the initiation of Australia, 1901-1919, Sydney.

Trainor, Luke, 1994. British imperialism and Australian nationalism: manipulation, conflict, and compromise in the late nineteenth century, Cambridge.

Ward, J.M., 1958. Earl Grey and the Australian Colonies, 1846-1857: A study of self-government and self-interest, Carlton.

White, R., 1981. Inventing Australia: Images and identity 1688-1980, Sydney.

Wicks, B. M., 1997. Understanding the Australian constitution: The plain words, Sandy Bay.

Williams, J., 1995. In search of the Federal Citizen: Andrew Inglis Clark and the 14th Amendment, Canberra. 


\section{Wegwerfen oder aussortieren? Das Deakinsche Bündel von "White Australia policy", Schutzzöllen und sozialer Marktwirtschaft 100 Jahre später Franz Oswald, Curtin University of Technology, Perth, Western Australia}

Die Bilanz von Australiens Erfahrungen im 20. Jahrhundert zu ziehen, ist nicht nur eine intellektuelle Übung für Historiker sondern ein sehr praktisches politisches Problem. Die ersten sieben Jahrzehnte des Jahrhunderts bewegte sich Australiens Politik in einem Rahmen, der etwa um 1900 vorgezeichnet wurde. Das bezieht sich nicht nur auf die Verfassung, die 1901 den Bundesstaat begründete, sondern auch auf die politischen Grundentscheidungen, die in den ersten Jahren danach fielen. Diese Entscheidungen sind mit dem Namen Alfred Deakin verbunden, Australiens zweitem Prime Minister, der als Führer der Protectionist Party, mit Unterbrechungen dreimal Regierungschef war. Das sogenannte "Deakinite settlement" erwies sich über Jahrzehnte hinweg als sehr stabil: "Seine Universalität lieferte das Bindemittel für achtzig Jahre nationaler Einheit und Fortschritt trotz seiner Defekte" (Kelly 1994:1), wurde aber seitdem gründlich in Frage gestellt. Am Anfang des 21. Jahrhunderts ist es unmöglich, die Deakinschen Politiken fortzusetzen, aber die Frage stellt sich ob Australien das ganze Deakinsche Bündel fallen lassen oder ob man selektiv vorgehen sollte.

Paul Kelly, prominenter Vordenker in der nationalen Tageszeitung "The Australian" und einflussreicher Autor, benennt fünf Deakinsche Politiken: die "White Australia policy", die Regulierung von Einkommen durch Schiedsgerichte ("arbitration"), die Schutzzollpolitik ("protectionism"), die paternalistische Rolle des Staates, sowie die Abhängigkeit vom britischen Weltreich (ibid.). Abgesehen von dem abwertenden Etikett "paternalistisch" für die Rolle des Staates als Sozialstaat und notwendiger wirtschaftlicher Entwicklungshelfer, hat Kelly damit langlebige Konstanten australischer Politik identifiziert.

Doch scheint jetzt das Ende dieser zweiten Phase australischer Geschichte gekommen: "Die erste Phase von der weissen Okkupation bis zum späten 19. Jahrhundert war die Gründungsperiode; die zweite, vom späten 19. Jahrhundert durch den größten Teil des 20. Jahrhunderts, war das Experiment des Aufbaus einer Nation angeleitet von den Ideen des 'Australian Settlement' nach der Föderation. Die Frage für die 1990er Jahre ist, ob die Marktphilosophie der 80er Jahre eventuell die dritte Phase bestimmt" (Kelly 1994:660). Damit gab Kelly dem Übergang zum Marktliberalismus der letzten zwei Jahrzehnte eine fast unwiderstehliche geschichtsphilosophische Weihe.

Eine vollständige Neuorientierung erscheint durchaus plausibel, da sich Australien im Innern wie auch in seinen Aussenbeziehungen im Laufe eines Jahrhunderts sehr verändert hat. Die größte Herausforderung für Australiens politische Institutionen ist, folgt man O. Hughes (1998:11), die langfristige Entwicklung der Wirtschaft. Diese ist charakterisiert durch sich verschlechternde "terms of trade" und die Tatsache, daß Australien sich nur auf sich selbst verlassen kann, seit Großbritannien 1973 der Europäischen Gemeinschaft beitrat. Australiens Platz auf einer Weltrangliste der Länder mit dem höchsten Lebensstandard hat sich langsam aber stetig nach unten bewegt. Gegen Ende des 19. Jahrhunderts genossen Australier "vielleicht das höchste" Pro-KopfEinkommen, und befanden sich zumindest in der Spitzengruppe. 1950 lag Australien an dritter Stelle hinter den USA und Canada, 1990 dagegen nur noch an 18. Stelle (Hughes:11). Kurz gesagt, das Land war Weltklasse darin, Güter zu produzieren, die andere Länder nur in abnehmenden Mengen oder zu abnehmenden Preisen brauchten. Und nach dem Ende des britischen Weltreichs muß sich Australien auf einem Weltmarkt zurechtfinden, auf dem immer mehr Tonnen Kohle, Weizen, oder Wolle exportiert werden müssen um die high-tech Produkte aus Japan, den USA oder Europa zu bezahlen.

Dieses wirtschaftliche Wettrennen gegen sich verschlechternde "terms of trade" darf nicht isoliert betrachtet werden, denn die Einstellung auf neue internationale Realitäten ist eng verbunden mit einer Neubestimmung der nationalen Identität, mit kulturellem Wandel, mit Modernisierung der 
politischen Institutionen, und mit einer Neuorientierung der Außenpolitik. Der frühere Prime Minister Malcolm Fraser benannte vier Aufgaben für australische Politik: Engagement mit Asien, die Versöhnung mit den australischen Ureinwohnern, Wettbewerbsfähigkeit und Modernisierung, sowie das Bekämpfen des "bigotten Hansonismus".

Will man diese umfassenden Modernisierungsaufgaben lösen, muß, aus Paul Kellys marktliberaler und kulturell liberaler Perspektive das ganze Deakinsche Erbe vollständig überwunden werden. Nichts ist davon zu retten. Jeder Blick zurück zu Deakin ist bestenfalls "sentimentaler Traditionalismus" (1994:660), schlimmstenfalls ein Rückfall in rassistische und ethnozentrische Vorstellungen, auf alle Fälle jedoch ein Hindernis für die notwendige Modernisierung von Australiens Wirtschaft, Politik und Kultur. Daß sich ein positiver Bezug auf Deakinsche Ansätze heute sowohl beim Nationalpopulismus der One Nation Party als auch auf der politischen Linken finden läßt, zeigt für Kelly nur, daß beide aus der Vergangenheit keine zukunftsweisenden Ideen entwickeln können.

Im Gegensatz zu Kellys Argumenten soll hier versucht werden, das Deakinsche Erbe selektiv auszuwerten, und zu fragen, was überwunden werden muß oder schon überwunden ist, und was heute noch, in neuer Form, fortgesetzt werden könnte.

Im folgenden werden die fünf Bestandteile des "Deakinite settlement" im einzelnen diskutiert, unter dem Gesichtspunkt ob man wirklich Kellys neoliberalen Argumenten folgen und die Deakinschen Politiken insgesamt verwerfen muß. Die rassistische "White Australia policy" ist eindeutig unannehmbar und wurde, zum Glück, vor gut drei Jahrzehnten abgeschafft. Die Abhängigkeit vom britischen Weltreich ist nach dem Ende dieses Reiches nicht mehr möglich, und jetzt kann man nur noch die Konsequenzen daraus ziehen. Die anderen drei Komponenten des "Deakinite settlement" können jedoch neu interpretiert und in einem neuen Kontext verwirklicht werden. Zwar bevorzugen Neoliberale vollständig deregulierte Arbeitsmärkte im Innern, Freihandel nach außen, sowie den Abbau der wirtschaftlichen Rolle des Staates ("developmental role") und des Sozialstaates. Zwar lehnt Paul Kelly diese Rollen des Staates als "state paternalism" ab. Doch sehen Teile des "Deakinite settlement", für einen europäischen Betrachter, aus wie Elemente einer australischen Version einer sozialen Marktwirtschaft, die nicht einfach in toto abgeschafft werden sollten.

Für Neoliberale wie Paul Kelly gibt es nur eine Methode der Modernisierung Australiens. Er bündelt die Bestandteile des "Deakinite settlement" in einem Paket. Dadurch wird jeder Versuch, gegen die Deregulierung des Arbeitsmarktes zu argumentieren, mit der "White Australia policy" und Pauline Hansons Nationalpopulismus in einen Topf geworfen. Jede Kritik an der neoliberalen Agenda der Regierungen der achtziger und neunziger Jahre wird als "sentimentaler Traditionalismus" kritisiert und in die Nähe des Hansonismus gerückt. Vielleicht ist es trotzdem möglich, das "Deakinite settlement" sorgfältiger zu analysieren, um es teils zu überwinden, teils zu beerben.

1. Die Verbindung mit dem britischen Weltreich war nicht beendet mit der Gründung des australischen Bundesstaates im Jahre 1901. Die Verfassung selbst wurde vom britischen House of Commons verabschiedet, als Commonwealth of Australia Constitution Act (9 Juli 1900). Damit war Australien kein unabhängiges Land, sondern ein Teil des Empire mit Selbstverwaltung. Für die meisten Australier war es möglich, sich als Brite und als Australier zugleich zu verstehen. Aus kulturellen, wirtschaftlichen und militärischen Gründen war eine Trennung vom Empire weder denkbar noch wünschenswert. Die verschiedenen Aspekte der "imperial connection" wurden jedoch mit der Zeit schwächer.

Im Zweiten Weltkrieg wurde es klar, daß Australien sich nicht mehr auf britische Hilfe verlassen konnte. Östlich von Suez war Großbritannien zu schwach und konnte die japanische Eroberung von Malaya und Singapur nicht verhindern. Unter Prime Minister John Curtin orientierte sich Australien seit 1941 eindeutig auf das Bündnis mit den USA. Australiens wirtschaftliche Verbindungen mit 
dem Empire endeten als Großbritannien 1973 der Europäischen Gemeinschaft beitrat. Das war das Ende der "imperial preferences" für landwirtschaftliche Exporte aus Australien und Neuseeland.

Die politische und verfassungsrechtliche Trennung entwickelte sich in identifizierbaren Schritten. 1930 wurde zum ersten Mal mit Sir Isaac Isaacs ein Australier zum Generalgouverneur ernannt, obwohl dieser Posten traditionell der britischen Aristokratie vorbehalten war. Das Westminster Statut von 1931, mit dem Großbritannien den Dominions größere Autonomie anbot, wurde von Canada und Südafrika schnell ratifiziert. Die australische Regierung war dagegen an soviel Unabhängigkeit nicht interessiert, da sich die (konservativen) Liberalen mit dem Empire identifizierten. Erst elf Jahre später ratifizierte die Labor-Regierung unter John Curtin das Dokument. Mit dem Australia Act von 1986 wurden die letzten rechtlichen Verbindungen mit Großbritannien abgeschnitten, mit einer Ausnahme: die Königin von England ist immer noch des Staatsoberhaupt von Australien.

Ein deutlicher Indikator für die Entwicklung einer separaten australischen Identität war die Einführung einer australischen Staatsbürgerschaft im Jahre 1948. Bis dahin wies der Reisepass alle Australier als britische Untertanen aus. Der Einzug von australischer Geschichte in Universitäten und Schulen seit den 1960ern trug auch zur kulturellen Überwindung der "imperial connection" bei.

Diese Komponente des "Deakinite settlement", die Abhängigkeit vom britischen Weltreich, hat ihre Bedeutung verloren. Das Vereinigte Königreich ist gewiß nicht mehr die Grundlage von Australiens wirtschaftlicher oder militärischer Zukunft.

2. Die "White Australia policy" gab Australiens Eliten die Gelegenheit, die Nation mit ethnozentrischen und rassistischen Argumenten $\mathrm{zu}$ integrieren. Für den durchschnittlichen Australier bot die "White Australia policy" die symbolische Gratifikation eines Überlegenheitsgefühls für jeden Angehörigen der "britischen Rasse" sowie den wirtschaftlichen Vorteil, daß potentielle Einwanderer anderer Ethnizität oder Rasse vom Wettbewerb um Arbeitsplätze ausgeschlossen waren. Die Wochenzeitung "The Bulletin" proklamierte die White Australia policy lange Jahre auf der ersten Seite: "The cheap Chinaman, the cheap Nigger (sic!), and the cheap European pauper to be absolutely excluded" (Horne 2001:15). Sie waren nicht britisch, sie wurden als Lohndrücker angesehen.

Noch 1960 verkündete "The Bulletin" den Slogan "Australia for the White Man", bis Donald Horne Herausgeber wurde und diese Praxis beendete. Schon vorher hatte sich Australien geöffnet durch die Entwicklung einer Einwanderungspolitik, die nach den Zweiten Weltkrieg zunehmend nichtbritische Europäer und dann auch Nichteuropäer zuließ. Schließlich wurde die White Australia policy um 1970s von Liberal und Labor Regierungen aufgegeben. Seitdem haben die allermeisten Australier die multikulturelle Realität akzeptiert. Das Aufleben eines ethnozentrischen Nationalpopulismus in Gestalt der One Nation Party seit 1998 ist nur ein begrenzter Rückfall in alte Stimmungen.

Die politische Kultur Australiens hat sich gewandelt und kulturelle Verschiedenheit ist weitgehend akzeptiert. Die nichtbritischen Einwanderer sind keine Gastarbeiter ohne politische Rechte, sondern Staatsbürger, die als Wähler Gewicht haben. Nach einem Jahrhundert haben Australiens Eliten andere Themen gefunden, um die Nation zu integrieren, und die Gewerkschaften verlangen nicht mehr eine rassistische Einwanderungspolitik um australische Arbeitsplätze zu sichern. Nationalpopulistischer Stimmenfang ist immer noch möglich, zum Beispiel, wenn die Regierung Howard Asylanten wie Kriminelle behandelt und sich als Schützer Australiens aufspielt. Nichtsdestoweniger, die White Australia policy endete unwiderbringlich vor gut dreißig Jahren.

3. Der Konflikt zwischen Schutzzollpolitik und Freihandel war so wichtig, daß sich am Ende des 19. Jahrhunderts politische Parteien um dieses Thema formierten. Die Free Traders erlitten eine entscheidende Niederlage, als die Protectionists unter der Führung von Alfred Deakin nach 1900 ein Bündnis mit der jungen Labor Party eingingen. Auf Kosten der Exporteure im Bergbau und in der Landwirtschaft, verbündeten sich die Industriellen, die für den Inlandsmarkt produzierten, mit den 
Gewerkschaften, die an sicheren Arbeitsplätzen interessiert waren. Seitdem waren Schutzzölle ein wichtiger Teil des "Deakinite settlement". Erst nachdem diese Frage entschieden war, konnten die zwei Fraktionen des australischen Kapitals zusammenkommen, um 1909 die "Fusion Party" zu gründen, die bald in "Liberal Party" umbenannt wurde. Von jetzt an betonten sie ihre gemeinsamen Interessen im Gegensatz zur Labor Party.

Siebzig Jahre später begann Australien mit dem Abbau von Schutzzöllen. Obwohl der Name "Liberal Party" eine Neigung zum Freihandel anzudeuten scheint, war es die Labor Party unter Prime Minister Whitlam, die zwischen 1972 und 1975 Importzölle reduzierte wogegen die Liberals und Nationals erst später auf diese Freihandelspolitik einschwenkten. Seitdem haben Regierungen beider großen Parteien Zölle herabgesetzt und in internationalen Foren Freihandel propagiert. Australien initierte die "Cairns Group" von Agrarexportländern, die in der Uruguay Runde (seit 1986) des GATT versuchten, die EU, USA und Japan zur Öffnung ihrer Agrarmärkte zu bewegen.

In Australien konzentrierte sich die Diskussion auf die Frage, wie schnell die Schutzzölle abgebaut werden sollten. Eine vollständige Reduktion aller Zölle auf Null wurde im Garnaut-Report vorgeschlagen (1989). Um das ideale "level playing field" zu erreichen, auf dem alle Wettbewerbshindernisse entfallen, sollte Australien vorangehen und einseitig Zölle abbauen, in der Hoffnung, daß andere Nationen nachziehen. Diese Vorschläge sollten Australiens Wirtschaft wettbewerbsfähiger machen und zugleich die großen Handelsblöcke EU, USA und Japan von den Wohltaten des Freihandels überzeugen. Die verarbeitenden Industrien konterten mit dem PappasCarter-Report (1989), der einseitige Zollreduktion als naive und schädlich zurückwies. Dies würde die australische Textilindustrie sehr schnell zerstören und bald auch zum Ende der Automobilindustrie führen. Deshalb sollten Zölle nur sehr langsam reduziert werden. Die Drohungen der Autoproduzenten General Motors-Holden, Ford, Toyota und Mitsubushi, ihre Fabriken in Australien zu schließen, hat den Freihandelsdogmatismus der Regierung Howard abgeschwächt. Dennoch wird es keine Rückkehr zum Deakinschen Protektionismus geben.

Ohne Deakins Protektionismus hätte Australien bis heute keine nennenswerte verarbeitende Industrie. Als Ergebnis des Protektionismus hat Australien eine verarbeitende Industrie, allerdings mit zum Teil prekärer Wettbewerbsfähigkeit. Man kann sich jetzt nicht mehr hinter Schutzzöllen verstecken, aber "industry policies" werden auch in einer Zeit des Freihandels nötig bleiben.

4. Die Regulierung des Arbeitsmarktes und der Arbeitswelt durch Schiedsgerichte, die "Conciliation and Arbitration Commissions", war ein einzigartiges Charakeristikum von Australien und Neuseeland. Die Aushandlung flächendeckender Tarifverträge wurde nicht den Tarifpartnern überlassen. Unternehmer und Gewerkschaften hatten dem Commissioner ihre Argumente vorzutragen wie in einem Gerichtshof. Die Entscheidung über Lohnhöhen und Arbeitsbedingungen wurde dann vom Commissioner getroffen: der "award" für eine ganze Industrie oder einen Berufszweig wurde "handed down", sozusagen von einer höheren Autorität gesetzt. Natürlich war der "award" immer eine wirtschaftliche Entscheidung, beeinflußt von Druck der Unternehmer und Gewerkschaften, dennoch hatte dieser quasi-gerichtliche Rahmen seine Auswirkungen. Für manchen Unternehmenr war "arbitration" eine unwillkommene Einschränkung seiner Entscheidungsfreiheit. Für kämpferische Gewerkschaften war "arbitration" eine staatliche Institution, die meistens den Unternehmern half. Für moderate Liberale dagegen, für die Labor Party und von der einflußreichen katholischen Soziallehre her gesehen, erreichte "arbitration" einen stabilen Klassenkompromiß, verhütete unproduktive Streiks und minderte Gewerkschaftsmilitanz.

Dieses Schiedsgerichtssystem veränderte die Auswirkungen des freien Arbeitsmarktes. Eine richtungsweisende Entscheidung von Justice Higgins im Jahre 1907 definierte den "basic wage" als ein Einkommen, das es einem Lohnempfänger erlaubt, eine Frau und zwei Kinder zu ernähren. Der wichtigste Aspekt dieser Entscheidung war, daß die Lohnhöhe nicht nur von Angebot und Nachfrage bestimmt wird. Bei der Lohnfestsetzung sollten auch soziale Gesichtspunkte mit einbezogen werden, insbesondere die Bedürfnisse der Arbeiter und ihrer Familien. Man kann sagen, daß diese Schiedsgerichte zu einer australischen Version der sozialen Marktwirtschaft beitrugen. 
Die Deregulierung der Arbeitsmärkte begann unter der Labor Regierung der Prime Minister Hawke und Keating (1983-1996) und wurde dann verstärkt fortgesetzt von den Liberals unter Prime Minister Howard (seit 1996). Die Rolle der Schiedsgerichte wurde reduziert. Die Tarifverträge für ganze Berufszweige oder Industrien wurden allmählich ersetzt durch Betriebsabkommen. Es wurde einfacher gemacht für Unternehmer, bei den Betriebsabkommen die Gewerkschaften von den Verhandlungen auszuschließen. Landesregierungen der Liberals wie auch einige größere private Unternehmen versuchten, diese Betriebsabkommen noch weiter $\mathrm{zu}$ individualisieren, indem sie $\mathrm{zu}$ "workplace agreements" mit einzelnen Beschäftigten übergingen.

Da die Labor Party seit 1996 in der Opposition ist, ist sie weniger dem Einfluß der neoliberalen Ökonomen im Finanz- und Wirtschaftsministerium ausgesetzt. Unter dem Einfluß der Gewerkschaften rückte Labor wieder etwas von den Betriebsabkommen und Einzelverträgen ab, um die Rolle der Schiedsgerichte wieder mehr zu betonen.

Dennoch scheint das vollständige Ende der Deakinschen Regulierung des Arbeitsmarktes unvermeidlich. Am 10. Januar 2001 entschied das Bundesgericht (Federal Court), daß die große Bergbaufirma BHP das Recht habe, ihren Beschäftigten individuelle Verträge anzubieten. Damit wurde die Möglichkeit der Gewerkschaften, kollektive Abkommen durchzusetzen, weiter beschnitten. Diese Entscheidung war wichtig genug, um das Thema eines Leitartikels in der führenden Tageszeitung zu werden ("Contracts win puts unions in their place", The Australian, 12. Januar 2001, p.10). Der sozialliberale Deakin von der Wende zum 20. Jahrhundert ist gewiß kein Leitbild für die Regierung des marktliberalen und sozialkonservativen Prime Minister John Howard am Anfang des 21. Jahrhunderts.

5. Was Paul Kelly als "state paternalism" abqualifizierte, ist die weitverbreitete Erwartung daß der Staat eine aktive wirtschaftliche und sozialpolitische Rolle spielen soll. In Australien hatte der Staat eine "developmental role" in der wirtschaftlichen Erschließung des Kontinents, weil privates Kapital oft nicht in der Lage war, profitabel in Infrastruktur zu investieren. In den USA konnten Privatunternehmen Eisenbahnen bauen und den Kontinent erschließen. Da Australien fast die gleiche Fläche aber weit weniger Einwohner hat, war der Eisenbahnbau nie profitable und wurde dem Staat überlassen. Zweitens hatte der Staat eine starke regulierende Rolle, besonders in der Vermarktung von Agrarprodukten (Australian Wheat Board, etc.). Drittens entwickelte der Staat auch eine Sozialstaatsrolle, besonders nach 1945, nach der Wirtschaftskrise und dem Zweiten Weltkrieg.

Keine dieser drei Rollen des Staates paßt heute auf die neoliberale Tagesordnung. Nachdem der Staat über hundert Jahre lang die Infrastruktur aufgebaut hat, ist es natürlich leichter, den Staat für überflüßig zu erklären.

Staatliche oder staatsnahe Unternehmen werden "corporatised", das heißt, zunehmend aus ihrer sozialen Verantwortung entlassen, um sich zu verhalten wie Privatunternehmen ("corporations"). Ist das erreicht, dann kann man sie auch gleich privatisieren. Das prominenteste Beispiel dafür ist Telstra, früher Telecom Australia. Dieses größte aller australischen Unternehmen wurde teilprivatisiert.

Die Konflikte über die Vollprivatisierung von Telstra machen die sozialen und politischen Kosten deutlich, die mit der neoliberalen Refromagenda verbunden sind. Telstra war schon "corporatised" noch ehe es privatisiert wurde. Zum Beispiel, Telstra konzentrierte sich auf die profitable Kommunkationsdienstleistungen in und zwischen Ballungszentren wie Sydney, Melbourne und Brisbane. Demgegenüber werden ländliche Kunden vernachläßigt, weil die australischen Entfernungen Dienstleistungen und Reparaturen sehr teuer machen. Ist Telstra ein Unternehmen, das nur seinen Aktionären verpflichtet ist, dann gibt es keinen Grund, auf dem Lande Verluste auf sich zu nehmen. Wenn aber Telstra eine soziale und politische Verpflichtung hat, zur Landesentwicklung beizutragen, dann müßen profitable Geschäftsbereiche dazu dienen, andere notwendige Aktivitäten durch "cross-subsidisation" zu unterstützen. 
Diese Frage hat unmittelbare politische Auswirkungen auf die Regierungskoalition von Liberals und ländlichen Nationals. In der Vergangenheit waren die Nationals für ländliche Wähler gegen schädliche Nebenwirkungen des freien Marktes angetreten: im Prinzip für die freie Marktwirtschaft, in der Praxis aber immer wieder für die Regulierung von Agrarmärkten und für staatliche Unterstützung von ländlichen Regionen. In den neunziger Jahren waren die Kompromisse zwischen Wirtschaftsliberalen und ländlichen Konservativen immer schwieriger geworden. Je mehr die führenden Politiker der Nationals die wirtschaftliberalen Ansichten ihrer Koalitionspartner übernahmen, desto mehr wuchs die Unruhe in den Kleinstädten und auf dem Lande.

"Die Nationals können nicht Teil der Howard-Koalition sein, ohne die Markt-Ökonomik zu akzeptieren, gegen welche Hanson ankämpft" (P. Kelly, "Truth cure for Hansonitis", The Australian, 24./25. Februar 2001, p.23). Dies ist genau die Situation, in der Pauline Hansons One Nation Party ihre Chance hat. Ländliche Wähler, vor allem Farmer und Kleinunternehmer, drücken ihren Unmut über die Auswirkungen neoliberaler Wirtschaftspolitik aus, indem sie gegen alles irritierende Neue protestieren, vor allem gegen den Wandel zur multikulturellen Gesellschaft.

Das "Deakinite settlement" vom anfang des 20. Jahrhunderts gibt es nicht mehr. Die "White Australia policy" wurde um etwa 1970s aufgegeben, die Einwanderungspolitik seit den 40er Jahren veränderte die Bevölkerung, und schließlich wurde "assimilationism" weitgehend durch multikulturelle Akzeptanz von Verschiedenheit ersetzt. Die Verbindung mit dem britischen Empire war am Ende, als Großbritannien 1973 der Europäischen Gemeinschaft beitrat. Und das Ende der drei wirtschaftlichen Komponenten der Deakinschen Politik wurde eingeläutet, als die Regierung Hawke 1983 den Wechselkurs des australischen Dollars zum "floating" freigab.

Was kommt nach dem Ende des "Deakinite settlement"? Wenn es nach Paul Kelly, dem Vordenker des "Australian", geht, wird Australiens Modernisierung wirtschaftliberale Elemente verbinden mit libertärem sozialen und kulturellem Wandel. Die Deregulierung der Wirtschaft würde begleitet von der Überwindung der White Australia Policy, dem Engagement mit Asien, der Versöhnung mit den Aborigenes, und der Trennung von der britischen Monarchie. Diese Kombination wäre am ehesten $\mathrm{zu}$ erwarten von Peter Costello, dem wahrscheinlichen Nachfolger John Howards als Regierungschef, sollten die Liberals die nächste Wahl gewinnen und an der Regierung bleiben. Gegenwärtig, unter Prime Minister John Howard, werden wirtschaftsliberale Ansätze mit sozialkonservativen Werten verbunden. Modernisierung bleibt unter Howard beschränkt auf Deregulierung der Wirtschaft und Zurücknahme des Staates aus wirtschaftlicher und sozialer Verantwortung. Dagegen kann sich Howard nicht von den Resten der "imperial connection" (der Monarchie) trennen. Es fällt ihm auch zu schwer, "sorry" zu sagen und zur Versöhnung mit den Aborigenes beizutragen, indem er vergangenes Unrecht anerkennt. Für Howard wäre das ein "black armband view of history", ein Bild der australischen Geschichte, das nur Negatives hervorhebt.

Sollte die Labor Party die Wahl Ende 2001 gewinnen, dann würde ein Prime Minister Kim Beazley, genauso wie der Liberale Peter Costello, das Engagement mit Asien, die Versöhnung mit den Aborigenes, und das republikanische Ende der "imperial connection" vorantreiben. In der Wirtschaftspolitik kann man vom Beazley dasselbe erwarten wie von seinem Freund Tony Blair oder von Gerhard Schröder: "neoliberalism lite" und Deregulierung versetzt mit Erinnerung an die australische Version sozialer Marktwirtschaft.

Den ethnozentrischen, rassistischen und britisch-imperialen Aspekten der Deakinschen Politik sollte niemand eine Träne nachweinen. Ob man zusammen mit diesen auch die sozialliberalen und sozialdemokratischen Elemente des Deakinschen Kompromisses hinauswerfen muß, ist eine andere Frage. Wenn man die Nebenwirkungen der neoliberalen Politik mit einkalkuliert, wie zum Beispiel den ländlichen Protest in Form des Nationalpopulismus der Pauline Hanson, fragt man sich, ob Modernisierung und Wettbewerbsfähigkeit sich nicht besser im Rahmen einer sozialeren Marktwirtschaft erzielen lassen. In einem Punkte hatte Deakin recht: den Arbeitsmarkt und die Landesentwicklung kann man nicht völlig dem freien Markt überlassen. Und als neue Einsicht kann man hinzusetzen: Im Vergleich zu Deakins Zeiten ist Australien eine offenere und tolerantere 
Gesellschaft geworden. Das sollte man nicht aus Spiel setzen durch den Versuch, Wettbewerbsfähigkeit durch Abbau von sozialer Sicherheit zu erreichen.

\section{Literatur}

Australian, The, 2001. Contracts win puts unions in their place, 12. Januar 2001, p.10.

Australian Manufacturing Council, 1990. The global challenge. Final Report of the Pappas Carter Evans and Koop/Telesis Study.

Garnaut, Ross, 1989. Australia and the northeast Asian ascendancy. Report to the Prime Minister and the Minister for Foreign Affairs and Trade, Canberra: AGPS.

Horne, Donald, 2001. Our greatest success: A willingness to change, The Australian, 1. Januar 2001, p. 15.

Hughes, Owen E., 1998. Australian politics, South Yarra: Macmillan Education.

Kelly, Paul, 1994. The end of certainty. Sydney: Allen \& Unwin. (Revidierte Ausgabe).

Kelly, Paul, 2001a. 100 Years: The Australian story. Sydney: Allen \& Unwin.

Kelly, Paul, 2001b. Truth cure for Hansonitis, The Australian, 24./25. Februar 2001, p. 23.

Souter, Gavin, 1992. Lion and kangaroo. Sydney: Pan Macmillan, (1st ed. 1976).

Wimmer, Adi, Hrsg., 1999. Australian nationalism reconsidered, Tübingen: Stauffenburg Verlag. 


\section{Die Föderation im Spiegel der zeitgenössischen Presse und Literatur Australiens}

\section{Cornelia Schulze, Universität Wuppertal}

Australien, der einzige Kontinent, der zugleich ein Staat ist, ist von Europa am weitesten entfernt. Dennoch rückt der "fünfte" und kleinste Kontinent nach den Olympischen Spielen 2000 in Sydney erneut in den Blickpunkt internationaler Aufmerksamkeit. Denn dieses Jahr feiert Australien das hundertjährige Jubiläum seiner Föderation und damit seiner staatlichen Eigenständigkeit. Am 1. Januar 1901 verkündete der erste australische Premierminister, Edmund Barton, die Gründung des "Federal Commonwealth of Australia": "There is a nation for a continent, and a continent for a nation" (Voigt 2000:70). Diese euphorischen Worte konnten nicht darüber hinweg täuschen, dass es sich um eine "schwere Geburt" handelte. Selbst wenn der Zusammenschluss zu einem einheitlichen Bundesstaat längst überfällig war, ging ihm eine jahrzehntelange öffentliche Diskussion voraus.

Besonders in einer Zeit des Umbruchs spielen die Medien eine zentrale Rolle. Auch in den entscheidenden Jahren vor der Föderation hatte die Presse einen bedeutsamen Anteil an den politischen (und mentalen) Veränderungen. Der Kampf um eine eigenständige Nation, die damit verbundene Loslösung vom Mutterland sowie die Entwicklung eines nationalen Selbstverständnisses hielt aber auch Einzug in die zeitgenössische Literatur. Die meisten Literaturgeschichten Australiens beschreiben die 1890er Jahre daher als den Beginn einer neuen Epoche, in der sich "australische" Literatur von einer kolonialen zu einer nationalen Literatur mauserte (Green 1961:2).

Anhand ausgewählter Beispiele lässt sich belegen, dass die Option einer "Föderation" schon früh in den Köpfen der Menschen existierte. Als wohl populärstes Beispiel lässt sich die offizielle heutige Australische Nationalhymne "Advance Australia Fair" anführen, die von Peter Dodds McCormick etwa 1878 komponiert (und gedichtet) wurde. In der zweiten Strophe heißt es: "In joyful strains then let us sing, / Advance Australia Fair. / Beneath our radiant Southern Cross / We'll toil with hearts and hands; / To make this Commonwealth of ours / Renowned of the all the lands..." (Voigt 2000:86). Überraschenderweise wird zwei Jahrzehnte vor der Föderation diese in der Nationalhymne vorweggenommen. Dieser Nationalstolz entsprach jedoch nicht der damaligen politischen Wirklichkeit. Um die politischen sowie literarischen Entwicklungen dieser Zeit verstehen zu können, müssen die australische Verfassung und Mentalität sowie die spezifischen Bedingungen der zeitgenössischen Presse und Literatur berücksichtigt werden.

Angesichts der heutigen Politikverdrossenheit europaweit erscheint das Tauziehen um die Föderation als ein Kraftakt, an dem nicht nur einzelne Spitzenpolitiker beteiligt waren - der Premier von New South Wales, Henry Parkes, gilt als "Vater der Föderation" -, sondern auch die Bevölkerung. Anders als in Deutschland herrscht in Australien die Wahlpflicht, und tiefgreifende Entscheidungen müssen vom Volk mitgetragen werden. Das jüngste Beispiel einer gesamtaustralischen Abstimmung war das Referendum am 6. November 1999 zur Republikfrage, deren Verwirklichung zur 100-Jahrfeier des Commonwealth anvisiert wurde. Doch die Mehrheit entschied sich für die Beibehaltung der Monarchie. Voigt bringt die Haltung der Australier auf den Punkt: "Warum sollten sie [die Australier] etwas abschaffen, was zahnlos ist und mit gepuderten Perücken keinem mehr etwas zu Leide tut? Toleranz ist eben auch ein Wesenszug der Australier" (Voigt 2000:82).

\section{Das Ringen um die Föderation im zeitgenössischen öffentlichen Diskurs}

Die Diskussion um die Vor- und Nachteile der Gründung eines Nationalstaates wurde nicht nur im öffentlichen Diskurs geführt, sondern auch in der zeitgenössischen Literatur. Im auslaufenden 19. Jahrhundert spiegelte die australische Presse das sich wandelnde Nationalbewusstsein, das in den Föderationsbemühungen mündete: 
"Die 1890er Jahre werden auch als die Jahre der Metamorphose und Synthese vom britischen Gefühl und der Kolonialidentität beschrieben. Das Nationalempfinden unterlag einem beeinflussbaren Wandel [...]. So war es doch in diesem Jahrzehnt überwiegend der Kolonialnationalismus, der zwar im Rahmen der Bundesgründung diskutiert wurde, aber bewusst als traditionsbindender Faktor beibehalten werden sollte." (Leutenecker 2000:1289).

Dieser Wandel wurde vor allem durch die Darstellung und Wertung der Ereignisse in der Presse und Literatur geprägt.

\section{Australiens Presse- und Literaturbetrieb}

In der zweiten Hälfte des 19. Jahrhunderts war der Literaturbetrieb im öffentlichen Diskurs eingebettet: Da erst in den 1890er Jahren ein lokales Verlagswesen entstand, nutzten die meisten Autoren die Gelegenheit, ihre Texte als Serie in einer der zahlreichen Zeitschriften zu veröffentlichen. Dieses Medium erforderte einen ausgeprägten Sinn für den Zeitgeist. Oftmals beteiligten sich die Autoren als (Mit-) Herausgeber oder verdingten sich als freie Journalisten. ${ }^{1}$ Die erste Zeitschrift für Literatur war das Hobart Town Magazin. Entstanden im Jahr 1834 war sie Medium für Kurzgeschichten, Essays und Gedichte. In Sydney gründete James Tegg seine Literary News, in der erstmals auch Illustrationen und Karikaturen veröffentlicht wurden. Dank der besseren Produktionsbedingungen und des technischen Fortschritts entstanden ab den 1850er Jahren zahlreiche Zeitschriften; einen wahren Boom erlebten die reformorientierten 1880er Jahre.

Die natürliche Affinität zwischen (politischem) Journalismus und Literatur verdeutlicht der Sydney Bulletin, Australiens meistgelesene und einflussreichste Zeitschrift für Politik und Literatur zwischen 1880 und $1910^{2}$. Im Jahr 1880 gründeten die Journalisten John Haynes und J.F. Archibald die Wochenzeitschrift, die sich zum bedeutendsten meinungsbildenden Organ etablierte. Der Bulletin hatte eine Doppelfunktion: Zum einen diente er als politisches Sprachrohr, zum anderen bot er talentierten Schriftstellern ein Forum:

"At first it seemed much like its predecessors and contemporaries [...]. But it was politically and economically chauvinistic and satirically opposed to colonial pretentiousness. It favoured the federation of the Australian colonies [...]. Its chief effects on literature were to popularize bush life as a subject, to encourage amateurs to write, and effectively to move the literary capital of Australia from Melbourne to Sydney." (Goodwin 1988:36)

Als eine Besonderheit der Zeitschrift galt die Red Page, ein Sonderteil für Literaturkritik, der sich aus der Sammlung von Buchrezensionen, "Books of the Day" und "Book Exchange" zu einer eigenständigen Kolumne entwickelte. Der maßgebliche Einfluss des Bulletin auf das Verständnis australischer Literatur ist untrennbar mit dem verantwortlichen Herausgeber (seit 1896) der Red

\footnotetext{
${ }^{1}$ Einzelfälle literarischer Betätigung gab es schon früh: Barron Fields veröffentlichte 1819 seine Sammlung von Gedichten First Fruits of Australian Poetry. Doch erst die Gedichtanthologie Bush Ballads and Galloping Rhymes (1870) zeigte ein typisch australisches Gesicht. Einen weiteren Höhepunkt stellten die Erzählungen von Henry Lawson dar, die er z.B. in While the Billy Boils (1896) zusammenstellte. Besondere Beachtung erhielt A. B. Paterson für seine Balladen, die er in The Man of the Snowy River and other Verses (1895) der Öffentlichkeit präsentierte. Den ersten Roman in der Weltliteratur über Australien schrieb in Deutschland Therese Hubel unter dem Titel Abentheuer auf einer Reise nach Neu-Holland (1793, erst 1966 ins Englische übersetzt), den ersten Roman auf australischem Boden schrieb Henry Savery mit Quintus Servinton. Die Einwanderer-Trilogie The Fortunes of Richard Mahony (1917-1929) schrieb eine Frau unter dem Pseudonym Henry Handel Richardson, das Schicksal einer Aboriginal Frau schilderte Katherine Susannah Prichard in Coonardoo (1929). Doch erst die Verleihung des Nobelpreises für Literatur an Patrick White im Jahr 1973 brachte australischer Literatur weltweite Anerkennung (vgl. Voigt 125-6).

${ }^{2} \mathrm{Ab}$ den 1920er Jahren nahm die Monopolstellung des Bulletin ab. Nach mehreren Besitzerwechseln übernahm die Australian Consolidated Press die Zeitschrift im Jahr 1960; in den 1970 ern erschien sie primär als ein Informationsmagazin, in den 1980ern und 1990ern entwickelte sie sich zu einem Lifestyle Magazin (vgl. The Penguin Australian Encyclopaedia, p. 75).
} 
Page verbunden (vgl. Kramer 1981:11). A.G. Stephens entwickelte einen spezifischen Schreibstil und forderte diesen auch von den Autoren, die im Bulletin veröffentlichen wollten: "Its [the Bulletin's] preference was for concise, even terse, fiction, firmly based on lived experience" (Wilding 1994:xi). Da der Großteil aller Kurzgeschichten der 1890er Jahre im Bulletin erschien, wurde dieser Stil zum Credo der Nachwuchsschriftsteller. Stephens ist auch ein neues literarisches Selbstverständnis zu verdanken, da er den Vergleich mit der Literatur aus Übersee nicht scheute und das Spezifische der australischen Literatur herauszuheben wusste:

"[W]hat country can offer to writers better material than Australia? We are not yet snug in cities and hamlets, moulded by routine, regimented to a pattern. Every man who roams the Australian wilderness is a potential knight of Romance; every man who grapples with the Australian desert for a livelihood might sing a Homeric chant of victory, or listen, baffled and beaten, to an Aeschylean dirge or defeat. The marvels of the adventures are our daily common-places. The drama of the conflict between Man and Destiny is played here in a scenic setting whose novelty is full of vital suggestion for the literary artist." (Stephens zitiert in Ackland 1993:249)

Ferner ermutigte Stephens jeden Australier, die Erfahrungen des australischen Daseins literarisch zu verarbeiten. Für ihn hatte Australien das Potential, eine Nationalliteratur zu generieren. Der Kampf zwischen Mensch und wilder Natur, der jenseits der getretenen Pfade stattfand, sollte dokumentiert oder fiktionalisiert werden. Diese Auffassung glich seine editorischen Interventionen wieder aus und führte zu einer demokratischen und egalitären Redaktionspolitik. Er verschaffte Schreibenden aus dem gesamten Kontinent Gehör, und der Bulletin avancierte zur "bushman's Bible" (Wilding 1994:xi). Stephens Ruf nach einer typisch "australischen" Literatur fand bei zahlreichen zeitgenössischen Autoren Anklang, vor allem bei A.B. Paterson und Henry Lawson, die in den 1890ern eine australische Variante der Ballade entwickelten:

"Here local settings became a crucial if superficial indicator of Australianness, while writers took their lead not from European balladry, but from colonial oral tradition. It was spiced with satire and refractory attitudes towards establishment values, and coloured by a sense of suffering and hardship." (Webby 2000:77)

Die Sehnsucht nach einem nationalen Selbstverständnis erwuchs vor allem aus Australiens Entwicklung zu einem geeinten Bundesstaat. Während zuvor Lokalpatriotismus gepflegt wurde, erforderten die politischen Bemühungen um die Föderation ein Umdenken, das sich auch in den literarischen Kreisen niederschlug:

"Writers like Henry Lawson, Miles Franklin and Joseph Furphy were more interested in depicting what was 'Australian' from an insider's point of view; the Australian landscape and ideas about the Australian 'national character' moved to the foreground in fiction around the turn of the century." (Webby 2000:105)

Anhand von ausgewählten Texten soll die Föderation im Spiegel der zeitgenössischen Literatur dargestellt werden. Zunächst werden Beispiele aus der Prosa, dann aus der Lyrik angeführt.

\section{Die Darstellung der Föderation und verwandter Themen in der Prosa}

Einige der bekanntesten fiktionalen Werke der Literatur dieser Epoche stammen aus dem Jahr der Föderation, wie z.B. Henry Lawsons Sammlungen von Kurzgeschichten The Country I Come From und Joe Wilson and His Mates, Miles Franklins autobiographischer Roman My Brilliant Career, William Hays Debütroman Stifled Laughter und Roderic Quinns Lyriksammlung The Circling Hearths. Eine repräsentative Auswahl der zeitgenössischen Kurzgeschichte verschafft uns Stephens The Bulletin Story Book, eine Sammlung von Prosawerken, die zuvor im Bulletin erschienen waren. Im Vorwort entwickelt er sein Verständnis von australischer (National-)Literatur, so dass es als ein zentrales Manifest zur zeitgenössischen australischen Erzählliteratur gewertet werden kann: 
"[...] the aim has been to make an interesting book. It has not been attempted to choose the best examples of literary style. Judged by a high canon, our most talented story-writers are still only clever students of the art of writing. A mere two or three have been able to earn a living by the profession of literature, and even these have been obliged to make the perilous compromise with journalism. So the stories and sketches which follow are usually the literary dreams of men of action, or the literary realisation of things seen by wanderers. Usually they are objective, episodic, detached - branches torn from the Tree of Life, trimmed and dressed with whatever skill the writers possess (which often is not inconsiderable). In most of them still throbs the keen vitality of the parent stem: many are absolute transcripts of the Fact, copied as faithfully as the resources of language will permit." (Stephens 1978:12)

Stephens verweist zunächst auf die nationalen Differenzen zwischen australischer und europäischer Literatur. Nicht der literarische Stil sei ausschlaggebend, sondern die Lebendigkeit der Darstellung der australischen Lebenswirklichkeit. Ferner erklärt er anhand der Entstehungs- und Produktionsbedingungen, warum australische Literatur nur bedingt in Reinkultur existent und erwünscht war. Zeitgenössische Autoren finanzierten ihre literarischen Eskapaden mit journalistischen Tätigkeiten und publizierten vor allem in Zeitschriften: Kurzgeschichten, Balladen, (politische) Lyrik, Pamphlete und alle Formen des Zeitungsjournalismus dominieren das Bild des damaligen australischen Literatur- und Kulturbetriebs. Als wesentliches Qualitätsmerkmal der australischen Geschichten nennt Stephens die Unmittelbarkeit und Episodenhaftigkeit der Darstellung. Aufgrund dieser Besonderheiten sollten die ersten literarischen Gehversuche nicht im direkten Vergleich mit den literarischen Traditionen Europas verglichen werden. In seinem Essay "For Australians" (1899) vertieft Stephens dieses Argument:

"And here in Australia, we have no temples, no ashes worth the name. We have still to make the history and create the legendary associations which are such a powerful binding force in national life. [...]. What a mass of record and tradition, of song and story, of memorable life and love and death, presses behind that England! Australia is meaningless by comparison, lacking the inspiration of the past. But is it not possible to catch meaning and inspiration from the future?" (Stephens 1978:395)

Aufgrund der fehlenden (europäischen) Tradition im eigenen Land muss sich Australien auf sein schöpferisches Potential besinnen, eine Zukunft erfinden, anstatt seine Vergangenheit in Geschichtsschreibung zu verbannen. Stephens verweist auf das große Potential der Neuen Welt und fordert Nationalstolz und Heimatliebe als verbindliche Erziehungsideale. Die Zukunft der Kolonie läge in den Händen der australischen Bevölkerung, "[...] it will be the fault of the people, not the land, if it is not one of the best countries in the world to live and to die in..." (1978:396). Die Qualität einer Nation hänge nicht von der Bevölkerungsdichte ab, sondern vom individuellen Beitrag eines jeden Bürgers. Daher endet sein Essay mit dem patriotischen Aufruf an die australische Bevölkerung, sich in Selbstoptimierung zu üben:

"Yet the future of Australia [...] rests upon the character of Australia's inhabitants. If it be the pride of every Australian boy to become a better man than his father, of every Australian girl to become a better woman than her mother [...] both the individual and the nation will surely have their reward." (1978:398-9)

Zwei Jahre vor der offiziellen Geburtsstunde der Föderation ruft Stephens die australische Bevölkerung auf, eine geeinte Nation zu bilden. In seinem Essay "For Australia" (1900) betonter, dass der Zusammenschluss der Staaten zu einer eigenständigen Nation ein Abstraktum bliebe, sofern sich diese Union nicht auch in den Köpfen und Herzen vollziehe:

"The Commonwealth, as an abstract idea, may give the satisfaction of the instinct of devotion to country; it can supply a target for a hitherto unsatisfied instinct; but beyond that, as an abstract idea, it gives nothing. [...]. There is no magical quality in this union: no 
millennium is to be reached by the declaration of a Federal Act in so many chapters and so many clauses. We suppose that the Commonwealth will give us greater scope for effort, a wider field for energy; but it is always our effort, our energy." (1978:403)

Stephens identifiziert Selbstverantwortung und persönliches Engagement als zugrundeliegende Prinzipien der angestrebten Staatsform. Nur dann erbringen, so Stephens, "[will it] be possible to justify all the enthusiasm to which the establishment of the Commonwealth has given birth by proving to nearly every individual in the community that Patriotism Pays" (1978:406).

Untersucht man das Verhältnis zeitgenössischer Prosawerke zur Föderation Australiens muss bedacht werden, dass viele Texte die Föderation zwar nicht explizit nennen, jedoch in der Atmosphäre aufkeimenden Nationalismus geschrieben wurden. So setzen sich Autoren, die im Föderationsjahr selbst oder kurz zuvor/danach publizierten, auf individuelle Weise mit Fragen zur typisch australischen Nationalität, Kultur und Lebensweise auseinander. Als Beispiele für unterschiedliche Formen, Australianness darzustellen, sollten vor allem diejenigen Werke berücksichtigt werden, die sich besonderer Beliebtheit erfreuten oder im Nachhinein zu außergewöhnlichem Erfolg führten. Dazu zählen Henry Lawsons Joe Wilson and His Mates (1901), Miles Franklins My Brilliant Career (1901) und Joseph Furphys Such is Life (1903). Als Henry Lawson seine achtzehn Kurzgeschichten im Jahr 1901 veröffentlichte, hatte er sich bereits als ein bedeutender australischer Autor etabliert:

"It was certainly Lawson who established the enduring model of a laconic minimalism of language that taut with irony and repressed emotion. His portrayal of the life of the Australian bush has made him a nationalist icon; and though he spent most of his life in poverty his portrait once adorned the ten-dollar note. His writing not only expresses the feel of Australia, it also contains an argument. It emerged from that brief flowering of radical optimism in the late 1880 s and early 1890 s, a period of intense trade-union activity, of revolutionary fervour and utopian optimism." (Wilding 1994:x)

Sein Verständnis von Australianness, das sich in Kameradschaft, Klassenegalitarismus und Stoismus ausdrückt, spricht aus jeder Zeile seiner Joe Wilson Geschichten: "Lawson's stories and characters have been a major influence in the construction of that traditional 'national identity' that only the last few decades have begun to dismantle..." (Webby 2000:106). Ironischerweise schrieb Lawson diesen typisch "australischen" Roman in London, wo er sich an die Bedürfnisse seiner britischen Leserschaft anpasste, die ihm zu diesem Zeitpunkt - zumindest geographisch - näher standen als seine treuen Landsleute: "One aspect of these stories that does give them a 'colonial' flavour is what John Barnes calls, an obvious awareness of a foreign audience in Joe Wilson's explanations - an aspect of the Joe Wilson stories that some Australian readers find irritating'" (Webby 2000:106). Dennoch hat Lawsons Präsentation des australischen Buschlebens das Australienbild geprägt.

Als zweites Beispiel für die Reflexion über das eigene Nationalempfinden kann My Brilliant Career von Miles Franklin angeführt werden. Es handelt sich um den Debütroman einer sechzehnjährigen Australierin, die schon zu diesen frühen Zeitpunkt die Gunst Henry Lawsons genoss. In seinem Vorwort lobt er Franklins lebendige Präsentation des Buschlebens und verleiht dem Roman das ersehnte Qualitätssiegel der Australianness: "I don't know about the girlishly emotional parts of the book [...]; but the descriptions of bush life and scenery came startlingly, painfully real to me, and [...] as far as they are concerned, the book is true to Australia - the truest I've ever read" (Franklin 1901:v). Oberflächlich betrachtet war das positive Urteil des legendären Lawson eine Ehre für die junge Schriftstellerin, doch es beinhaltet auch chauvinistische Tendenzen, die für den Männlichkeitsethos (und der damit einhergehenden Entwertung der Weiblichkeit) Australiens zu Beginn des 20. Jahrhunderts symptomatisch sind. In Franklins Roman dient die australische Landschaft eher als Hintergrund für das Portrait einer unangepassten jungen Australierin, die bemüht ist, sich in der männlich-dominierten Gesellschaft zu behaupten. Im Verlauf des Romans entwickelt sich eine Liebesgeschichte, die unerfüllt bleiben muss. Franklin kombiniert in ihrem 
semi-autobiographischen Roman Elemente der Romance mit einer Parodie dieses Genres. Der Roman endet mit einer pathetisch-patriotischen Anerkennung der Leistungen der Australier: Die Männer bewähren sich im Ringen mit der wilden Natur, die Frauen aufgrund ihrer opferwilligen und arbeitsamen Mentalität (Kindler 1988: 781). Dieser Schlussakkord des Romans mag Lawson mit Franklins "mädchenhaftem" Interesse an den inneren Konflikten der Protagonistin versöhnt haben. Immerhin teilen Rezensent und Autorin einen sentimentalen Nationalstolz. So bekennt Sybylla: "I am proud that I am an Australian, a daughter of the Southern Cross, a child of the mighty bush" (Franklin 1901:231). Als Franklin My Brilliant Career im Jahr der Föderation veröffentlichte, traf sie den Nerv einer im Wandel begriffenen Gesellschaft, die sich mit Blick auf die Vereinigung nach einer verbindlichen Definition von Australianness sehnte.

Als drittes Beispiel für zeitgenössische Prosa bietet sich Such is Life (1903) von Joseph Furphy an. Furphys Hauptwerk verdankte seine Veröffentlichung dem persönlichen Einsatz von A.G. Stephens. Trotz dieser einflussreichen Protektion fand der experimentelle Roman bei den zeitgenössischen Lesern zunächst wenig Beachtung. Erst allmählich erkannte man die Bedeutung des Buches, das heute als "one of the great masterpieces and challenges of Australian literature" gilt (Webby 108). Besonders auffällig ist der fragmentarische, sprachreflektorische Schreibstil mit zahlreichen literarischen Anleihen, der Elemente des postmodernen Erzählens antizipiert. Die Konzeption und Ausführung von Such is Life zeigt Furphys bewusste Ablehnung des australischen Abenteuerromans in der literarischen Tradition von Henry Kingsley oder Rolf Boldrewood. Such is Life gilt somit als "significant for the part it played in turning Australian fiction away from the colonial romance" (Wilde et al. 1985:305). Furphy zeigt auch keine Berührungsängste mit dem literarischen Erbe Englands: er integriert zahlreiche Shakespeare- und Bibelzitate und macht parodistische Anspielungen auf den viktorianischen Roman. Der Zeitpunkt der Veröffentlichung war insofern gut gewählt, als Furphys aggressiv-nationaler Ton die Stimmung reflektierte, aus der Australien als unabhängige Nation zwei Jahre zuvor hervorgegangen war.

Grundsätzlich zeigen die besprochenen Prosawerke die intensive Suche der Autoren nach typisch "australischen" Themen und Ausdrucksformen und transportieren die Atmosphäre aufkeimenden Nationalbewusstseins. Seit der 1890er Jahre stand nicht die Vermittlung eines Australienbildes an Nicht-Australier im Vordergrund, sondern das Schaffen eines nationalen Selbstverständnisses. Je "australischer" ein Werk auf Kritiker und Leser wirkte, desto enthusiastischer fielen die Werturteile aus.

Im Gegensatz zur Prosa, in der die Föderation nicht direkt zur Sprache kam, findet man in der zeitgenössischen Lyrik zahlreiche Anspielungen auf die Föderationsbemühungen. Zudem hatte Lyrik in der utilitaristischen Gesellschaft Australiens einen relativ geringen Stellenwert, so dass aktuelle Bezüge zur australischen Wirklichkeit eine qualitative Aufwertung erzielten. Im Folgenden sollen Beispiele aus der zeitgenössischen Lyrik vorgestellt werden, in denen die Vor- und Nachteile der Föderation diskutiert wurden. Denn der Ruf nach politischer und literarischer Selbstständigkeit der 1890er Jahre kulminierte in der Entstehung populärer Balladen und satirischer Gedichte, die das wachsende Nationalbewusstsein reflektierten und daran beteiligt waren, eine gemeinsame nationale Identität zu formen (vgl. Webby 2000:74ff).

\section{Die Darstellung der Föderation und verwandter Themen in der Lyrik}

W.T. Goodge zählt zu den prominenteren Gegnern der Föderation. Norman Lindsay bezeichnete ihn als "one of Australia's best writers of light verse" (Wilde et al 301). Im Jahr 1899 publizierte Goodge seine Anthologie Hits! Skits! And Jingles!, eine Sammlung komischer Gedichte, die von skurrilen oder tragik-komischen Buschpersönlichkeiten bevölkert sind. In seinem satirischen Gedicht "Federation" karikiert er die Föderationsbemühungen als ein populistisches Unternehmen mit zweifelhaftem Ausgang:

Let us sing of Federation

('T is the theme of every cult)

And the joyful expectation 
Of its ultimate result. [...].

We shall drink to desperation,

And be quite the soberest nation

We'll be desperately loyal

Unto everything that's royal,

And be ultra-democratic

In a matter most emphatic.

We'll be prosperous and easeful,

And pre-eminently peaceful,

And we'll take our proper station

As a military nation! [...].

Hier fungiert Goodge als Sprachrohr der Gegner des Federation movements, da er die gängigen Bedenken artikuliert. Im Mittelpunkt der Kritik steht die angestrebte Verfassung mit ihrer sonderbaren Mischung aus der britischen parlamentarischen Regierungsweise und dem USamerikanischen Föderalismus. Während Befürworter der Föderation die Verbindung von Integration und Gleichheit bei gleichzeitiger Wahrung der Eigenständigkeit der einzelnen Kolonien lobten, fürchteten andere paradox anmutende Konsequenzen: Australien sollte zwar aus seiner kolonialen Abhängigkeit befreit werden, doch die britische Krone würde das offizielle Staatsoberhaupt bleiben. So könne die verfassungsmäßige Verbindung zu Großbritannien kaum dazu beitragen, die Nabelschnur zum Mutterland endgültig zu durchtrennen. Das Gedicht endet mit der Vorhersage:

\section{We'll adopt all fads and fictions \\ And their mass of contradictions \\ If all hopes are consummated \\ When Australia's federated; \\ For the Federation speeches \\ This one solid moral teach us - \\ That a pile of paradoxes are expected to result!}

(Murray 1986:;67)

Mit ironischem Augenzwinkern sagt Goodge die euphorischen Worte der politischen Redner zu Ehren der Föderation voraus. Das Gedicht endet mit dem eindringlichen Appell an den Leser, nicht in den Gesang auf die Föderation einzustimmen, sondern kritische Distanz zu der politischen Rhetorik einzuhalten. ${ }^{3}$

Im Folgenden werden Beispiele von Autoren lyrischer Werke angeführt, die sich offiziell für eine gesamtaustralische Nation einsetzten. Als imposante Gegenstimme zu Goodge kann A.B. Paterson angeführt werden. Auch er ist ein Beispiel für die Verquickung literarischen und journalistischen Schreibens. Er war Herausgeber der Sydney Evening News und des Australian Town and Country Journal sowie des Sydney Sportsman. Paterson gilt als der herausragendste Volksdichter Australiens, dessen Vision des pastoralen Australien (Australian Arcadia) in seinen berühmten Balladen entwickelt und verbreitet wurde. Seine erste Monographie, The Man from Snowy River and Other Verses (1895), war innerhalb einer Woche ausverkauft. Mit seinem "Waltzing Matilda" erschuf er nicht nur das bekannteste australische Nationallied, sondern auch einen musikalischen Exportartikel. Ähnlich wie Furphy kreierte er in seinen Werken ein ganzes Arsenal australischer Volkshelden. Er gehörte zu der wachsenden Gruppe radikaler Nationalisten der 1890er Jahre, die sich für ein geeintes, starkes Australien einsetzte. Patersons Vielseitigkeit und schillernde Persönlichkeit erlaubte ihm eine enorme Medienwirksamkeit und Präsenz im zeitgenössischen öffentlichen Leben. Sein Gedicht, "Song of the Federation", schrieb er zu Ehren der australischen Föderation. Das geeinte Australien wird darin als wunderschöne Frau beschrieben: "She was

\footnotetext{
${ }^{3}$ Goodge könnte auf den Premier von New South Wales, Henry Parkes, angespielt haben, der im Jahr 1889 eine mitreißende Rede zugunsten einer nationalen Regierung für den gesamten Kontinent hielt (vgl. Voigt 2ßBß:70).
} 
beautiful as morning,/With the bloom of the roses on her mouth, / Like a young queen lavishly adorning/Her charms with the splendours of the South" (Paterson 1946:183-4). Trotz dieser Idealisierung dringen auch skeptische Untertöne durch, da die Föderation Kind des Kalten Krieges war: "As the nations sat together, grimly waiting- / The fierce and ancient nations battle-scarred- / Grown grey in their lusting and their hating, / Ever armed and ever ready keeping guard, / Through the tumult of their warlike preparation / And the half-stiffed clamour of the drums / Came a voice crying, 'Lo, a new-made Nation, / To her place in the sisterhood she comes!'" (1946:183-4). Es gehörte zur zeitgenössischen Propaganda radikaler Nationalisten, dass Australien trotz seiner geringen Bevölkerung eine machtvolle Nation werden könne, da die Australier im Ringen mit der unzähmbaren Natur eine robuste Mentalität erworben hätten. Bevölkerungsstärkere Nationen, so das Argument, könnten auf diese innere Stärke nicht zurückgreifen, auch wenn sie schon viele Kriege geführt haben mögen: "Hark, and ye shall hear my children singing / Their war-song in countries far away. / They are strangers to the tumult of the battle, / They are few, but their hearts are very strong, / Twas but yesterday they called unto the cattle, / But they now sing Australia's marching song" (1946:184). Mit diesem kämpferischen Ton fing Paterson den radikalen Patriotismus seiner Zeit ein.

Ein weiterer Befürworter der Föderation war William Gay, aus dessen Feder die bekannten Gedichte "Australian Infelix," "Australian Federation" und "Australia 1894" stammen, mit denen er der Föderation seine geistige Unterstützung zusagt. Im ersteren beklagt er die immer wieder fehlschlagenden Bemühungen, die einzelnen Kolonien zu einem Nationalstaat zu vereinen. So zeigt er seine wachsende Ungeduld: "How long, O Lord, shall this, my country, be / A nation of the dead? / How long shall /They / Who seek their own and live but for the day, / My country hinder from her destiny?" (Gay 1911:102). In seinen Augen verhindert die egoistische Politik der einzelnen Kolonien das Entstehen einer machtvollen Nation. Erst als solche könne sich Australien aus seinem selbstverschuldeten Unglück befreien. Er bittet Gott um Unterstützung bei dem Unterfangen, die Lokalpatrioten zum Schweigen zu bringen, damit Australien endlich den Status einer eigenständigen Nation erhält. In seinem Gedicht "Australian Federation" argumentiert Gay für eine Föderation:

From all division let our land be free,

For God has made her one: complete she lies

Within the unbroken circle of the skies, And round her indivisible the sea

Breaks on her single shore; while only we,

Her foster children, bound with sacred ties

Of one dear blood, one storied enterprise,

Are negligent of her integrity.-

Her seamless garment, at her Mammon's nod,

With hands unfilial we have basely rent, With petty variance our souls are spent,

And ancient kinship under foot is trod:

$O$ let us rise, united, penitent,

And be one people, - mighty, serving God!

(Gay 1911:103)

In diesem Gedicht präsentiert Gay Australien als ein vollkommenes Werk Gottes. Erst die europäischen Bewohner hätten den Kontinent in einzelne Kolonialstaaten zerstückelt. Gay sieht es als die moralische Pflicht des australischen Volkes, das Land wieder in seinen ursprünglichen, von Gott gewollten Zustand zu versetzen. So beschwört er am Ende des Gedichts eine Nation, ein Volk herauf. In "Australia 1894" personifiziert Gay Australien als Königin, die mit kritischem Blick Zwietracht und Vereinzelung in ihrem Reich entdeckt: "Yet as she gazes o'er the plains that 
lie/Fruitful about her throne, she sighs full / sore / To see the barriers Greed has builded high, / Dividing them who brothers were before, / When still they dwelt beneath a sterner / sky / And heard the thunders of a wilder / shore" (1911:114). Durch Menschenhand und aufgrund von Raffgier wurden Zäune errichtet, wo zuvor ein einziges Land existierte. Dieses Mal verweist Gay auch auf Gefahren von außen, denen nur eine starke Nation etwas entgegenzusetzen habe. Ähnlich wie Paterson leitet er die Bedeutsamkeit eines geeinten Australien aus innerer wie außenpolitischer Notwendigkeit ab.

Edward Dyson, geboren 1865 in Victoria, zählte ebenfalls zu den Befürwortern der Föderation. Als unkonventionelles Vorwort stellte er sein Gedicht "Australia" an den Anfang seines Gedichtbands 'Hello Soldier!' Khaki Verse (1919) und räumte ihm so einen Ehrenplatz ein. Die sieben Strophen des Gedichts sind in Form eines Dialogs des Sprechers mit Australien konzipiert. Anfangs bemerkt das lyrische Ich anerkennend, dass Australien aus einer Art Koma erstanden zu sein scheint: "Australia [...]. You've done some rousing things of late, / That lift you from the simple state / In which you chose to vegetate" (Dyson 1919:3). Doch dann drückt Dyson die Notwendigkeit aus, dass sich Australien aus der imperialistischen Umklammerung befreien muss, um tatsächlich selbständig zu werden: "At length you need no sort of crutch, / You stand alone, you've voted 'much' - / Get busy and behave as such" (1919:3). England und andere Nationen hätten den neuen Status Australiens längst erkannt: "You've given all the world to know / Your proper standing as a foe, / And hats are off, and rightly so" (1919:3). Ungeduldig fordert er Beweise ein, an denen erkennbar ist, dass Australien "flügge" geworden ist: "Now have you proved it to your own?" (1919:3). Eindringlich fordert er Australien auf, die nagenden Selbstzweifel endgültig zu überwinden. Die letzte Strophe ist die Krönung des Gedichts mit starkem Appellcharakter:

Australia, life your royal brow,

And have the courage of the pride,

Audacity becomes you now,

Be splendidly self-satisfied.

No land from lowliness and dearth

Has won to eminence on earth

That was not conscious of its worth.

Nur der Wandel von der veralteten Kolonialidentität zu offen bekundetem Nationalstolz kann die "schlafende Prinzessin" erwecken. Eigenverantwortung scheint der Schlüssel, da Australien nicht im klassischen Sinn durch einen "Kuss" erweckt werden kann, sondern sich selbst erschaffen muss.

Ein weiterer Repräsentant herausragender australischer Lyrik mit patriotischem Grundton ist John Farrell. In dem von der Zeitung Sydney Morning Herold publizierten A Century of Journalism wird Farrell zusammen mit Henry Lawson und A.B. Paterson aufgeführt, da die Werke dieser Autoren als besonders "australisch" bezeichnet werden können: "The work of each is Australian to the core" (Tearle 1971:147). Farrell neigte dazu, australische Politik und Geschichte in seine lyrischen Werke hineinzutragen. Beispielsweise schrieb er zu Ehren von Queen Victorias Diamantenem Jubiläum im Jahr 1897 die Ode "Ave Imperatrix". Zur Geburtsstunde der Föderation sang ein Chor aus tausend Stimmen Farrells "The Hymn of the Commonwealth". Thema der Hymne ist die Bruderschaft zwischen den einzelnen australischen Staaten und ihre gemeinsame Zukunft als vereinte Nation. Er erkannte jedoch, dass die einzelnen Kolonialstaaten nicht einfach zusammenwachsen würden und verwies in seinem Gedicht "Gone Wrong" auf das fehlende nationale Zusammengehörigkeitsgefühl:

Alas! We still are states at heart -

Our chief desires are still the same.

We swaggered in the better part,

Then lapsed, to play the same old game

For keeps; to do the best we can

For our own gain, by force or stealth - 


\section{Ourselves before our fellow-man \\ Our wealth before Our Commonwealth.}

Farrell macht eine Beobachtung, die noch über Jahrzehnte nach Ausrufen der Föderation Geltung hatte. Er selbst fühlte sich Australien gegenüber zutiefst verpflichtet. Als aus Anlass des hundertjährigen europäischen Besiedelung Australiens der Picturesque Atlas of Australasia (1888) publiziert wurde, schrieb er sein wohl berühmtestes Gedicht "Australia," das auch in der Föderationsausgabe des Daily Telegraph erschien. Es endet mit den Zeilen:

O dear and fair! awakened from thy sleeping

So late! The world is breaking into noon;

The eyes that all the morn were dim with weeping

Smile through the tears that will cease dropping soon!

Thine have no tears in them for olden sorrow,

Thou hast no heartache for a ruined past;

From bright to-day to many a bright to-morrow

Shall be thy way, O first of lands and last!

(beide Gedichte zitiert in Tearle 1971:154)

Ähnlich wie in den vorherigen Beispielen wird Australien als "Sleeping Beauty" dargestellt, die gerade erst erwacht ist. Seine koloniale Vergangenheit und die fehlende Nationalidentität können überwunden werden, sofern es gelingt, sich von nostalgischen Gedanken zu befreien. Hier spielt Farrell wieder auf die Schwierigkeiten vieler Australier an, sich von ihrem provinziellen Lokalpatriotismus zu befreien und Australien als eine geeinte Nation zu verstehen, für die es sich zu engagieren lohnt. Seine Fähigkeit, Australien in seiner Ganzheit zu begreifen und zu vermitteln, beeindruckte auch Arthur W. Jose, den Herausgeber der History of Australasia (1913), der Farrells "Australia" und "Australia To England" zum "high water mark of our patriotic song" zählt (zitiert in Tearle 1971:154). Farrell versuchte wie einige seiner Zeitgenossen, Australien auf dem schwierigen Weg in die staatliche (und vor allem psychologische) Eigenständigkeit zu unterstützen.

Für viele Befürworter der Föderation Australiens war die Möglichkeit der Selbstgestaltung des Landes der motivierendste Grund, sich in den 1890er Jahren verstärkt für eine vereinte Nation einzusetzen. Der Begriff "Föderation" wurde zu einem Synonym für nationale Selbstbestimmung und die aktive Beteiligung Australiens auf der Schaubühne der Welt. So mauserte sich Australien von einer ehemaligen Strafkolonie zu einer unabhängigen Nation. Im Zeichen der fortschreitenden Globalisierung kommt Australien im 21. Jahrhundert wieder eine veränderte geopolitische Rolle zu. Es wird lohnenswert sein, die australische Presse und Literatur daraufhin zu untersuchen, ob und auf welche Weise sich Australien im öffentlichen Diskurs als "global player" präsentieren wird.

\section{Bibliographie}

Ackland, Michael, Hrsg., 1993. The Penguin book of $19^{\text {th }}$ century Australian literature. Melbourne: Penguin.

Dawson, Sarah, Hsg., 1990. The Penguin Australian encyclopaedia. Melbourne: Penguin.

Dyson, Edward, 1919. 'Hello Soldier!' khaki verse. Melbourne: Alex. M'Kingley.

Franklin, Miles, 1901. My brilliant career. Sydney: Angus \& Robertson.

Gay, William, 1911. The complete poetical works of William Gay. Melbourne: Thomas Lothian.

Green, H.M., 1961. A history of Australian literature pure and applied. Bde. I \& II. Sydney: Angus \& Robertson.

Goodwin, Ken. 1988. A history of Australian literature. London: Macmillan Education.

Kindlers neues Literaturlexikon, 1988, hsg. von Walter Jens, München: Kindler. [Band 5]

Kramer, Leonie, Hsg., 1981. The Oxford history of Australian literature. Melbourne: OUP. 
Leutenecker, Gerd, 2000. "Die 1790er, 1890er und 1990er Jahre: Australien an drei Jahrhundertschwellen." in: Rudolph Bader, Hsg., Australien auf dem Weg ins 21. Jahrhundert: Bilanzen -Standortbestimmungen - Visionen. Tübingen: Stauffenburg Verlag. 119-142.

Murray, Les, Hrsg., 1986. The new Oxford book of Australian verse. Melbourne: OUP.

Paterson, A.B., $1946^{13}$. The collected verse of A. B. Paterson. Sydney: Angus \& Robertson.

Stephens, A.G., 1978. A.G. Stephens : Selected writings. Hrsg. Leon Cantrell. Melbourne: Angus \& Robertson.

Tearle, Sheila, B.T. Dowd, 1971. John Farrell: poet, patriot and journalist, Journal of the Royal Australian Historical Society. 57(2 ). 143-159.

Voigt, Johannes H., 2000. Australien. München: C.H. Beck'sche Verlagsbuchhandlung.

Webby, Elizabeth, Hrsg., 2000. The Cambridge companion to Australian literature. CUP.

Wilberforce, A., C. Jose, H.J. Carter, Hrsg., 1925. The Australian encyclopaedia. Bd. I. Melbourne: Angus \& Robertson.

Wilde, W.H., J. Hooton, B. Andrews, Hsg., 1985. The Oxford companion to Australian literature. Melbourne: OUP.

Wilding, Michael, 1994. The Oxford book of Australian short stories. Melbourne: OUP. 


\section{Beiträge aus Forschung und Lehre}

\section{Willkommen nur fernab der neuen Heimat? Die Beschäftigung von osteuropäischen Ärzten in Papua and New Guinea 1950-1970: Eine historische Fallstudie zur Problematik hochqualifizierter Zuwanderung}

\section{Henriette v. Holleuffer, Pinneberg}

"Vielleicht ist der König von England der große Häuptling in England, aber vor Ort bin ich der Chef, und ich sage Dir, daß Du hier bleiben sollst" (Haszler 1967:38). Wertschätzung artikuliert sich gelegentlich in ungewöhnlicher Form: Anfang der fünfziger Jahre erfuhr der ungarische Mediziner Dr. Karl (Charles) Haszler auf überraschende Weise Anerkennung für sein fachliches Können durch einen Häuptling der Luluai im australisch verwalteten Teil New Guineas. Als der Chirurg in Mount Hagen (New Guinea) das Flugzeug bestieg, um in Port Moresby eine neue Aufgabe als Amtsarzt der australischen Regierung zu übernehmen, sah er sich zu seinem Erstaunen 2000 Stammeskriegern der Luluai gegenübergestellt. Sie erwarteten ihn auf dem provisorischen Flugfeld, um seine Abreise zu verhindern:

"(...) meine Taschen wurden aus dem Flugzeug genommen, meine Hände und Arme wurden von beiden Seiten gepackt und ich wurde gefaßt. Allein der Rede von Reverend Döring an der Startbahn und (anderen) Überzeugungskräften war es zu verdanken, daß ich freigelassen wurde." (1967:38).

Die aktuelle migrationswissenschaftliche Debatte vermag ohne die Lehren der Wanderungsgeschichte nicht auszukommen. Letztere ist dank ihres welthistorischen Ansatzes und aufgrund ihrer globalen Ausrichtung voll unerschöpflicher Beispielhaftigkeit (vgl. Kulischer/Kulischer 1932). Ende der vierziger und Anfang der fünfziger Jahre experimentierte das australische Department of Immigration mit der Aufnahme neuer Gruppen von Einwanderern. Mit der kabinettsinternen Billigung des Entwurfs zur "Revised Immigration Policy" vom April 1947, der auf Betreiben von Minister Arthur A. Calwell zur Diskussion gestanden hatte, bahnte die LaborRegierung unter Joseph Benedict Chifley der Aufnahme osteuropäischer Displaced Persons (DPs) den Weg. Damit sprengte sie den bis zum Ende des Zweiten Weltkrieges üblichen engen Rahmen australischer Einwanderungspolitik. Mit den entwurzelten Opfern der nationalsozialistischen Herrschaftspraxis in Europa kamen erstmals Heimatsuchende in größerer Zahl auf den Fünften Kontinent, die dem im Kern angelsächsisch strukturierten Bevölkerungskorpus der australischen Gesellschaft entgegenstanden. Die Zuwanderung dieser überwiegend osteuropäischen Personengruppen, denen als Überlebenden der nationalsozialistischen Konzentrationslager, als ehemaligen Zwangsarbeitern und Kriegsgefangenen im Deutschen Reich ein Sonderstatus im Statut der International Refugee Organization (IRO) zuerkannt worden war, bahnte der Ausformung eines "multi-ethnischen" Australien erste Pfade (abgedruckt in: Djonovich 1946:99). Nicht alle Displaced Persons, denen der australische Staat zwischen 1947 und 1952 Aufnahme gewährte, entsprach dem von Arthur A. Calwell apostrophierten und avisierten Idealbild des "Sohnes harter Arbeit mit der schwieligen Hand" (Conference on Displaced Persons, 18.7.1947). Ein auffallend hoher Anteil an DP-Siedlern hatte eine akademische Ausbildung vorzuweisen. Die meisten von ihnen verschwiegen oder modifizierten jedoch ihren beruflichen Werdegang bei den Befragungen durch die australischen Auswahlteams mit Blick darauf, daß es demonstrativ gesetzte Präferenzen für solche Arbeitnehmer gab, die eine nicht-akademische Vorbildung besaßen.

Die folgenden Ausführungen enthalten eine historische Fallstudie zur Problematik der Zuwanderung von hochqualifizierten Arbeitnehmern. Anhand eines ausgewählten Beispiels, der Beschäftigung der New Australian Doctors in Papua and New Guinea in den Jahren zwischen 1950 und 1970, wird exemplarisch dargelegt, wie Australien zwischen 1947 und 1956 auf den Zustrom 
akademisch vorgebildeter Neuansiedler mit tertiärem Berufsabschluß reagierte, - zu einem Zeitpunkt, als das Department of Immigration mit neuen Zielvorgaben an der Modifikation der überkommenen australischen Einwanderungspraxis formulierte. Als die australische Regierung der IRO 1947 die Aufnahme und Beschäftigung größerer Kontingente von europäischen Displaced Persons in Aussicht stellte, war nicht abzusehen, daß im Rahmen dieser Initiative bis 1952 rund 182.000 New Australians auf den Fünften Kontinent kommen würden. In dieser Bilanz enthalten war eine Zahl von ungefähr 370 DP-Medizinern (Kunz 1975:22-23). Gemäß offizieller Schätzung war die Zahl der als heimatlos registrierten europäischen DP-Ärzte zwischen 1945 und 1949 auf annähernd 3000 Personen angewachsen (1975:7). Die meisten von ihnen hatten eine umfassende akademische und praktische Ausbildung. Viele besaßen Spezialkenntnisse, u.a. im Feld der Tropenmedizin.

Es muß als Verdienst der IRO angesehen werden, daß sich diese Institution in besonderer Weise um eine Neuansiedlung der durch Krieg und Diktatur entwurzelten Mediziner bemühte. Zu diesem Zweck hatte die Internationale Flüchtlingsorganisation das sogenannte Professional Medical Register eingerichtet, in dem sich ausschließlich solche DP-Mediziner registrieren lassen konnten, deren berufliche Werdegänge durch eine von der IRO autorisierte Prüfungskommission verifiziert worden waren. Damit eröffnete die Flüchtlingsorganisation allen auswanderungswilligen Ärzten, die infolge verlorengegangener Identitätsnachweise und Diplome besondere Hemmnisse bei der beruflichen Reintegration zu erwarten hatten, reale Chancen auf eine adäquate Neubeschäftigung in ihrem Feld. Gleichzeitig sicherte die IRO potentiellen Aufnahmestaaten zu, daß bei allen im Professional Medical Register gelisteten Ärzten die medizinische Kompetenz "bestätigt und nachgeprüft" worden war (IRO Professional Medical Register 1948:3). Die Flüchtlingsorganisation hatte seit September 1947 verstärkt auf die mangelnden Zukunftsperspektiven dieser hochqualifizierten Berufsgruppe hingewiesen (Kunz 1975:8). Zu jenem Zeitpunkt leisteten viele der entwurzelten Doktoren und Professoren gering bezahlte Dienste in den europäischen Flüchtlingslagern der IRO oder in den Kliniken der Besatzungszonen Deutschlands und Österreichs.

Der Welt offerierten sich die heimatlos gewordenen Ärzte als vielseitig verfügbare Ressource von Fachkräften, deren breites medizinisches Wissen und deren umfassender Erfahrungsschatz kaum zur Kenntnis genommen wurden. Kontrovers gestalteten sich die berufsständischen Debatten u.a. in Kanada oder Australien darüber, ob den europäischen Medizinern sogleich nach ihrer Neuansiedlung in Übersee gestattet sein sollte, eigenverantwortlich zu praktizieren. Früh zeichnete sich $a b$, daß eine adäquate Beschäftigung heimatloser Mediziner in Übersee gleich nach der Ansiedlung nicht zu erwarten war. Einige wenige amerikanische, kanadische und australische Befürworter, die sich für eine Aufnahme ausländischer Mediziner in ihren Ländern aussprachen, wiesen auf bestehende Ungleichgewichte zwischen einheimischer Bevölkerungsgröße und verfügbarer Ärztezahl hin, um den schnellen Zugriff auf diese Fachgruppe sicherzustellen (vgl. The Ottawa Journal, 8.1.1948) Noch zu Beginn des Jahres 1948 reagierte das australische Department of Health ablehnend auf den Vorstoß der IRO und der World Health Organization (WHO), eine internationale Initiative zur Aufnahme der osteuropäischen Mediziner zu begründen: Ärzte und Zahnärzte, die "nur ausländische Diplome" besäßen, würden in Australien für eine klinische oder niedergelassene Tätigkeit nicht registriert werden (Kunz 1975:8). Der Anstoß für eine australische Initiative kam aus dem Department of Immigration. Ende September 1948 gab Brigadier F. G. Galleghan, der Leiter der Australian Military Mission in Deutschland, der IRO den Hinweis, daß eine Registrierung von ausländischen Ärzten ohne akademische Wiederholungsprüfung in den australischen Einzelstaaten nicht erfolgen würde, es jedoch grundsätzlich möglich sei, die heimatsuchenden Mediziner im australisch verwalteten Territorium Papua and New Guinea einzusetzen. Hier sei unter der Verantwortung des Medical Board of the Territory of Papua-New Guinea eine Registrierung legal; lokal auf das "external territory" begrenzt, konnten Anrechte auf eine Arbeitserlaubnis in Australien hingegen nicht erworben werden (Kunz 1975:8-9). Im Dezember 1946 hatte Australien sein altes (Völkerbund-)Mandat für das Treuhandgebiet New Guinea durch die Vereinten Nationen bestätigt bekommen. Erst im September 1975 erlangte Papua and New Guinea als administrative Einheit die Unabhängigkeit. 
Im November 1948 ließ der verantwortliche Amtsarzt der australischen Militärmission in Berlin, Dr. H. Graham Andrew, Galleghan ein Memorandum zukommen, in dem er sich für die Einwanderung von DP-Ärzten aussprach (Kunz 1975:9-10). Zwar sollte potentiellen Kandidaten angesichts der scharfen Opposition durch die British Medical Association in Australia (seit 1962: Australian Medical Association) zunächst nur eine vage Garantie auf eine spätere Tätigkeit als "hospital worker" zugesichert werden. Doch legte Dr. Andrew dem australischen Einwanderungsministerium nahe, im Rekrutierungswettbewerb mit den USA diese wichtige Ressource hochqualifizierter Arbeitnehmer für die Zukunft zu sichern. Er gab zu bedenken, daß der geplante Aufbau eines Australian National Health Service in absehbarer Zeit einen erhöhten Bedarf an Medizinern erfordern würde, der durch den aktuellen Bestand an australischen Ärzten nicht zu decken sei. Das Memorandum enthielt eine Reihe von Vorschlägen, auf welche Weise sich Australien die Fachkompetenz der europäischen DP-Mediziner nutzbar machen könnte, ohne sogleich deren Registrierung als approbierte Ärzte durchsetzen zu müssen. Allein das New GuineaProjekt bot einen realistischen Ausweg aus dem Dilemma, auf die Politik der berufsständischen Abschottung durch die British Medical Association in Australia Rücksicht nehmen zu müssen, ohne sich den Zugriff auf ein frei verfügbares Potential fertig ausgebildeter Spezialisten zu verbauen.

Um genügend Anträge auswanderungswilliger DP-Ärzte auf Neuansiedlung in Australien zu erhalten, lancierte das Department of Immigration die Streuung von Informationen über einen akuten Medizinermangel in Australien sowie über den geplanten Aufbau eines modernen National Health Service auf dem Kontinent - interne Hinweise darauf, daß die Neuankömmlinge zunächst keine fachlich angemessene Beschäftigung finden würden, wurden zwar nicht verschwiegen, ließen dem Optimismus der Heimatsuchenden gleichwohl breiten Raum zur Deutung (Kunz 1975:13-18). Eine Befragung aus dem Jahr 1953 zeigte auf, in welchem Umfang und in welcher Art die angeworbenen Mediziner vor ihrer Ausreise aus Europa über ihre bevorstehende Verwendung auf dem Fünften Kontinent informiert gewesen waren. Grundlage der Datenaufnahme, die der Soziologe Egon F. Kunz 1975 auswertete, waren die Antworten von rund 200 DP-Ärzten, die die Unregistered Doctors' Association in Victoria zu diesem Fragenkomplex erbeten hatte (1975:1718). Danach ergab sich folgendes Bild: Die ausreisewilligen Fachkräfte hatten weder klare noch übereinstimmende Informationen über die Aussichten einer beruflichen Tätigkeit in Australien erhalten. Zwar gaben $33 \%$ der Befragten an, daß ihnen kein konkretes Versprechen hinsichtlich einer beruflich adäquaten Anstellung gegeben worden war, da die Situation für medizinische Fachkräfte aus Nicht-Commonwealth-Ländern traditionell problematisch gewesen sei. Doch immerhin 24\% der Befragungsteilnehmer waren mit der Vermutung eingewandert, daß in Kürze eine Änderung der australischen Gesetzgebung ihnen eine ärztliche Tätigkeit erlauben würde, die dem Ausbau eines modernen Gesundheitssystems dienen sollte. Zehn Prozent der Neuankömmlinge waren in der festen Gewißheit eingereist, daß sie unmittelbar nach ihrer Ansiedlung als Ärzte beschäftigt würden; sieben Prozent spekulierten auf "medizin-nahe" Tätigkeiten als LaborFachkräfte oder Forschungsassistenten. $21 \%$ der rund 200 befragten New Australian Doctors dokumentierten einen anderen Optimismus: Sie glaubten, nach Abschluß der zweijährigen Kontrakt-Zeit ohne Auflagen eigenverantwortlich medizinisch tätig sein zu können. Zur Ableistung dieser Dienstverpflichtung war jeder Neuansiedler verpflichtet gewesen, der unter der australischen Einwanderungs-Initiative Ende der vierziger und Anfang der fünfziger Jahre in die südliche Hemisphäre kam. Bei Nicht-Erfüllung des zweijährigen Kontrakts drohte den Zuwanderern die Ausweisung. Die restlichen Antworten aus der Befragungsgruppe enthalten folgende Informationen: Drei Prozent der Antwortgebenden rechneten mit einer sofortigen Verwendung als Schiffsärzte bei der Überfahrt nach Australien; zwei Prozent hatten befürchtet, ihren DP-Status durch die Zurückweisung eines australischen Aufnahmeangebots zu verlieren, obwohl sie eine Auswanderung in die USA bevorzugt hätten; nur wenige der Befragten hatten rechtzeitig realisiert, daß zumindest die Wiederholung von zwei klininischen Jahren der Weiterbildung nötig sein würde, um eine Registrierung als approbierter Arzt zu erlangen.

Charakteristisches Merkmal des australischen Kontrakt-Modells blieb der Tatbestand, daß unabhängig von der fachlichen Vorbildung und dem professionellen Werdegang des New 
Australian eine Beschäftigung in zumeist un- oder minderqualifizierten Tätigkeitsbereichen erfolgte. Als Kontrakt-Partner und Arbeitgeber wies der australische Staat somit auch hochqualifizierten Akademikern Tätigkeiten als Arbeiter oder Hilfskräfte in Fabriken, Servicebetrieben oder Unternehmen des Commonwealth zu. Das Erscheinungsbild des australischen Kontrakt-Systems wies anfangs groteske Züge auf:

"Jemand, der 1949 die Holden-Fabrik in Fishermen's Bend in Melbourne besuchte, konnte dort Akademiker und die 'Intelligentsia' aus ganz Zentral- und Osteuropa antreffen. Der 'neue' australische Wagen, der 'Holden', wurde produziert von New Australians, unter der Beaufsichtigung einiger australischer Vorarbeiter. Anwälte, Juristen, Tierärzte, Doktoren, Architekten, sie alle bauten das Auto Australiens" (Haszler 1967:35).

Charles Haszler, der als New Australian Doctor 1964 zum ersten Präsidenten der neugegründeten Papua and New Guinea Medical Society berufen wurde, lieferte mit dieser Schilderung einen Augenzeugenbericht voller Symbolkraft. Der New Australian Haszler leistete seine Kontrakt-Zeit als Arbeiter bei General Motors Holden ab:

"Es herrschte keine glückliche Stimmung, viel Unzufriedenheit und Murren äußerte sich - jeder wollte von dort weg. Die New Australian-Arbeiter hatten beinahe immer einige Muttern und Bolzen über, sowie ein paar Drähte vom Instrumentenbrett freiliegend. Die Old AustralianVorarbeiter beherrschten nicht die Fähigkeit, die bejahrten Anwälte, Augenärzte und Tierärzte in wenigen Stunden in die Kunst, richtig mit Preßluftgeräten zu arbeiten, einzuweisen" (1967:35).

Die Erfüllung des Kontrakts sicherte den Neuankömmlingen ein erstes materielles Auskommen zu. Als problematisch erwies sich gleichwohl der branchenfremde Einsatz und die sich während dieser Verpflichtung herauskristallisierende Erkenntnis, daß nur eine langwierige akademische Requalifikation die ersehnte australische Approbation als Arzt sichern würde. Berichte hierüber sowie über die Option, in bestimmten Staaten der USA nach einer medizinischen Einarbeitungsphase leichter zur Anerkennung ihrer europäischen Abschlüsse zu gelangen, lenkten den Strom heimatloser Ärzte aus Osteuropa verstärkt in die USA. Aus der Rückschau bleibt gleichwohl anzumerken, daß auch in Nordamerika, insbesondere in Kanada, die Anerkennungsverfahren gleichfalls problembefrachtet blieben, da hier die Abschottung durch berufsständische Organisationen ähnlich negativen Einfluß nahm. Wie der Sozialwissenschaftler Egon Kunz in seinen umfassenden Befragungen über die Beschäftigung von New Australians mit akademischer Vorbildung herausfand, arbeiteten die zugewanderten Mediziner auch nach Abschluß ihres Kontrakts noch mehrheitlich in branchenfremden Hilfstätigkeiten, u.a. als Arbeiter. Bei Tätigkeiten in medizinischen Beschäftigungsfeldern beschränkte sich der Einsatz der vollausgebildeten Ärzte auf untergeordnete Dienste als Krankenpfleger, Laborassistenten, Röntgenassistenten, Apothekengehilfen oder Putzhilfen (Kunz 1975:100; Auswahlgruppe: Tab.5). Die außerordentliche materielle und psychologische Belastung, der diese Gruppe heimatsuchender Immigranten ausgesetzt war, verstärkte sich bei den hochqualifiziert Ausgebildeten, die auf berufliche Anerkennung hofften, dramatisch: Bis Anfang 1956 wurden der australischen Öffentlichkeit mindestens fünf Selbstmorde unter den New Australian Doctors bekannt. Vor diesem Hintergrund griff der Sydney Morning Herald die Problematik am 1. Februar 1956 auf:

"Viele Leute fordern eine Überprüfung der offiziellen Haltung gegenüber diesen Ärzten. Mehr als 400 New Australian Doctors sind in dieses Land gekommen, aber nur ungefähr 60 haben es geschafft, (berufliche) Anerkennung zu finden. Die Leute fragen jetzt, ob es nicht schlicht unmenschlich ist, diesen - gewöhnlicherweise mittellosen - Wesen zu sagen, daß sie ohne Hilfe an die nächst erreichbare Universität gehen sollen, um dort die letzten drei Jahre der medizinischen Ausbildung abzuschließen. Wie (über)lebt der Mensch während der Zeit?"

Das New Guinea-Projekt erfaßte nur wenige der osteuropäischen Ärzte. Die Rekrutierung für dieses Vorhaben begann Ende 1949 (Haszler 1967:36). Verantwortlich für die Durchführung des Plans war Dr. J. T. Gunther, der Anfang 1946 zum Director of Public Health for Papua and New Guinea im australisch verwalteten Territorium berufen worden war. Seine Aufgabe sollte es sein, im Territorium eine moderne medizinische Basisversorgung aufzubauen. Dieses Ziel wurde nicht 
zuletzt mit Blick darauf verfolgt, daß die soziale und politische Entwicklung von Treuhandgebieten seit Ende des Zweiten Weltkriegs international verstärkt Kontrollen unterzogen wurde. Weder im Vereinigten Königreich noch in Australien fanden sich 1949/50 genügend Mediziner, die bereit gewesen wären, in New Guinea zu arbeiten (zum folgenden s. Haszler 1967:36-39 sowie Kunz 1975:52-55). Die verhältnismäßig geringe Anzahl junger australischer Ärzte, die Ende der vierziger Jahre ihre Approbation erlangte, bevorzugte es, in Ballungszentren zu wirken. Dr. H. Graham Andrew, der vor seiner Berufung als Amtsarzt an die Australian Military Mission in Berlin für die United Nations Relief and Rehabilitation Administration (UNRRA) tätig gewesen war, trug seine positiven Erfahrungen in der Zusammenarbeit mit den sogenannten DP-Ärzten in australische Fachkreise. Als Dr. Gunther diese Empfehlung aufgriff und sich in Regierungskreisen um eine Anwerbung der heimatlosen Kollegen bemühte, wurde ihm zunächst eine abschlägige Antwort zuteil. In einem weiteren Anlauf erhielt er, nicht zuletzt in Zusammenarbeit mit Einwanderungsminister Arthur A. Calwell, das Signal zur Umsetzung des New Guinea-Plans. Es kam schließlich zu einer konstruktiven Abstimmung zwischen dem Department of Health und dem Department of Immigration: DP-Ärzte, die sich zu einem Einsatz in Papua and New Guinea bereiterklärten, durften hier unter Anerkennung ihrer akademischen Abschlüsse sofort eine medizinische Tätigkeit aufnehmen.

Daraufhin besuchte Dr. Gunther verschiedene Aufnahmelager für Immigranten, in denen geeignete New Australians vor Ort Auswahlinterviews unterzogen wurden. Eine handverlesene Schar von 35 (Scragg 1964:30 berichtet abweichend von 37) Medizinern unterschiedlicher nationaler Herkunft erhielt die Genehmigung zur amtsärztlichen Tätigkeit in New Guinea. Ihrer Volkszugehörigkeit nach gliederte sich diese erste Gruppe in folgender Weise auf (Haszler 1967: 36-37): Ungarn (12); Esten, Letten, Litauer (9); Polen (8); Tschechen (5); Jugoslawen (1). Zwischen 1951 und 1957 nutzten weitere Flüchtlings-Ärzte das australische Angebot für eine Tätigkeit in Papua and New Guinea: Ungarn (10); Deutsche (3); Rumänen (2); Tschechen (4). Der besondere Zuwachs der ungarischen Medizinergruppe war Begleitumstand der politischen Aufstände im Heimatland dieser New Australians.

Welche Aussagen lassen sich über die erste Abordnung von DP-Ärzten, die 1950 Papua and New Guinea erreichte, machen? Die Musterauswahl war mit Bedacht vorgenommen worden: So befanden sich unter den ersten Bereitwilligen einige bevorzugt ausgewählte Ungarn, mit denen Dr. Andrew zuvor in einem IRO-Hospital im bayrischen Föhrenwald erfolgreiche Patientenarbeit geleistet hatte. Die Vorbereitung auf die tropenärztliche Tätigkeit hatte System. Alle neuen Ärzte erhielten eine dreimonatige Schulung an der Australian School of Pacific Administration (ASOPA) in Mosman, Sydney. Hier besuchten sie Intensivkurse in Englisch. Darüber hinaus erhielten sie spezielle Unterweisungen in den Feldern der Anthropologie, Geschichte und Geographie New Guineas. Weitere Unterrichtseinheiten beschäftigten sich mit der australischen Territorialverwaltung und der Lebensweise in den Tropen. Nach Beendigung dieser Schulung folgte ein einmonatiger Anschlußkurs in Port Moresby. Die Mediziner mußten ihre Familien zunächst in Australien zurücklassen. Bis zum 15. April 1950 hatte jeder der neuen australischen Amtsärzte einen eigenen Verwaltungsposten auf New Guinea zugewiesen bekommen; einige bezogen Stationen, auf denen erstmals offiziell ein Arzt praktizierte. Hier leisteten die New Australian Doctors unter primitiven Bedingungen wichtige Pionierarbeit für den Aufbau einer modernen Gesundheitsversorgung in Papua and New Guinea. 


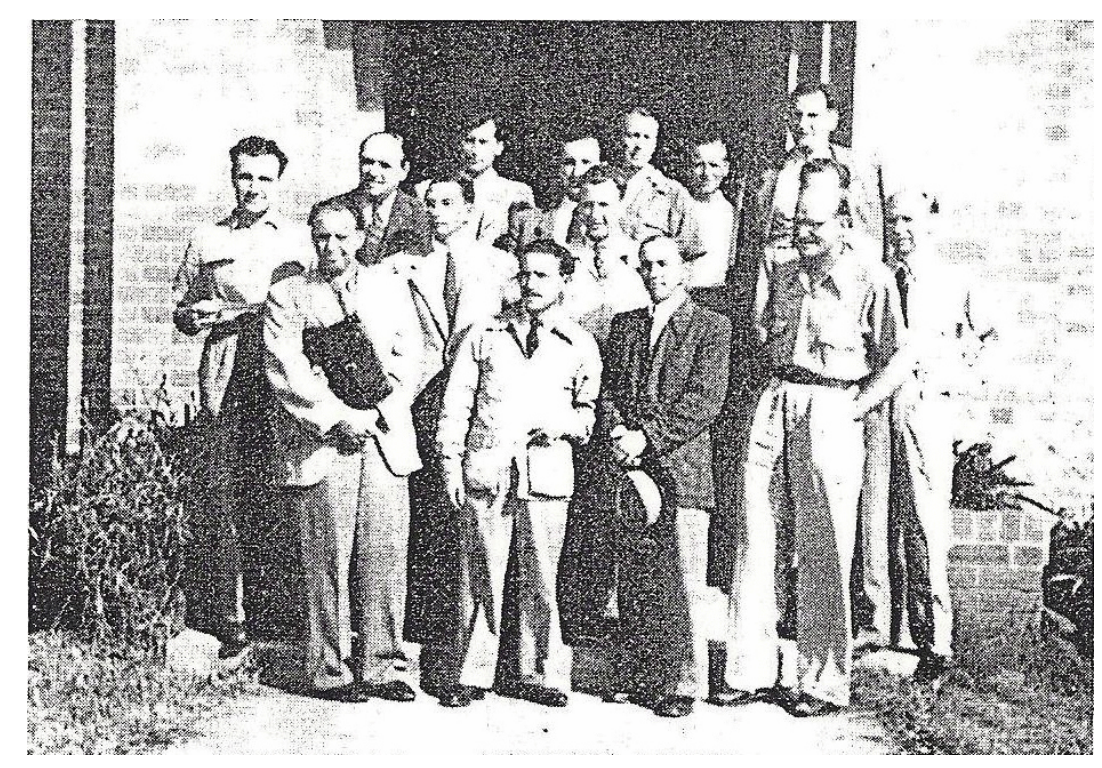

Mitglieder der ersten Gruppe von New Australian Doctors. Das Bild zeigt die Ärzte in Sydney kurz vor ihrer Abreise nach New Guinea im Jahr 1950. (Quelle: Papua and New Guinea Medical Journal)

Das medizinische Wirken der osteuropäischen Ärzte gewann besondere Bedeutung vor dem Hintergrund der australischen Entwicklungsarbeit in Papua and New Guinea. Es waren die fünfziger Jahre, in denen das von Australien verwaltete Territorium einen entscheidenden Innovationsschub hinsichtlich seiner medizinischen Basisversorgung erhielt. Das entwicklungspolitische Engagement, das Dr. Gunther in seiner Funktion als australischer Director of Public Health for Papua and New Guinea vorantrieb, vollzog sich in einem klar gesteckten Rahmen. Zwei wichtige Zielsetzungen waren Bestandteil eines Plans gewesen, dessen Verwirklichung erst durch die Beschäftigung der aus Übersee zugewanderten Ärzte möglich wurde: So war es vorrangig darum gegangen, zum einen die Gesundheitsversorgung im Territorium zu professionalisieren sowie zum anderen für eine verbesserte medizinische Schulung der einheimischen Kräfte zu sorgen. Diesen Vorhaben entsprechend umrahmten zwei Daten die Initiativen des Entwicklungsprojekts: 1949 erfolgte die Entscheidung, die osteuropäischen Mediziner in den Immigration and Reception Centres des Landes zu rekrutieren. 1956 gewann der Medical Training Plan for Papua and New Guinea, in dem die Gründung des Papuan Medical College avisiert wurde, erste Konturen. Letzteres nahm seit 1958 strukturelle Formen an. Als eine der am wenigsten entwickelten Regionen der Welt profitierte Papua and New Guinea umfassend von dem Einsatz der aus Osteuropa stammenden Mediziner. Fernab ihrer neuen Heimat unterwarf Canberra diese Einwanderergruppe in konstruktiver Nutzung ihrer hohen Qualifikation einem politischen Kalkül: Durch den Einsatz der New Australian Doctors in Papua and New Guinea erwuchs den australischen Einwanderungsplänen gleichermaßen außenpolitische wie entwicklungspolitische Bedeutung. Die Regierung in Canberra erhielt internationale Anerkennung für ihre soziale Aufbauarbeit im Treuhandgebiet. Sie beugte damit zugleich einer möglichen Kritik an der australischen Verwaltung des Treuhandgebiets vor. Möglich wurde dies u.a. dank der Mitarbeit der New Australians am "Modernisierungswerk" im Territorium.

Kompetentes und motiviertes Personal ist der Schlüssel zu einem gut funktionierenden Gesundheitssystem. Vor 1950 besaß Papua and New Guinea nur einen sehr kleinen Bestand an ausgebildetem Fachpersonal. Mit den Aufgaben der medizinischen Versorgung war es überfordert. Diese Erkenntnis von offizieller Seite eilte den Eindrücken der Neuankömmlinge voraus. Dr. Gunther hatte unmittelbar vor Ankunft der New Australian Doctors zu bedenken gegeben: "In dieser Hinsicht (der ärztlichen Behandlung der Kranken) werden wir weitestgehend behindert durch unzulängliches Personal" (Dr. J. T. Gunther, Address to the Administration, Missions Conference 1949). Die einreisenden Ärzte bestätigten 1950 diese Einschätzung:

"Wir alle hatten uns zu vergegenwärtigen, daß wir die einzig verfügbare medizinische Hilfe für viele Tausend Menschen waren. 1950, als ich in Mount Hagen arbeitete, war ich der einzige Arzt 
zwischen Wewak, Daru und Goroka, und (ich wußte, daß) wenn ich diese Menschen im Stich ließe, dort niemand mit einem medizinischen Examen gewesen wäre, um zu helfen" (Haszler 1967:39).

Von der Existenz einer medizinischen Infrastruktur konnte zu Beginn der fünfziger Jahre nur in Ansätzen gesprochen werden:

"Die Missionen im Hochland hatten zu der Zeit keinen Amtsarzt in ihrem Stab, nur ein paar Schwestern. (...) Als wir Port Moresby erreichten und das alte Ela Beach Hospital sahen, traf uns ein leichter Schock, wie auch immer, den größten Schlag bekam ich, als ich in Mount Hagen ankam und zum ersten Mal das Krankenhaus auf der anderen Seite der Landebahn entdeckte" (Haszler 1967:39).

In ihrer Funktion als Public Health Officers fiel den Neuansiedlern ein breitgefächertes Aufgabengebiet zu. Verwaltungstechnische Dienste und medizinische Eingriffe aus allen Bereichen gehörten zu den Betätigungsfeldern:

"Das Wichtigste war, daß wir uns in die Rolle des öffentlichen Gesundheitsbeamten einfanden, der zugleich klinische Arbeit leistet. Es dauerte ziemlich lange, bis wir realisierten, daß wir es in diesem Land nicht nur mit einzelnen Kranken $\mathrm{zu}$ tun hatten, sondern daß wir uns mit (grundlegenden) Problemen der öffentlichen Gesundheit befaßte." (Haszler 1967:38).

Von Ärzten, die ihren Beruf zuvor an den vielfältig spezialisierten Universitäts- und Fachkliniken Europas erlernt, ausgeübt oder gelehrt hatten, wurden bei ihrem neuen Einsatz in New Guinea fachübergreifende Fähigkeiten der Allgemeinmedizin und Chirurgie abverlangt sowie Spezialkenntnisse vor allem in den Bereichen der Gynäkologie, Dermatologie und Tropenmedizin erwartet. Der ungarische Arzt Charles Haszler erinnerte sich in einem Fachartikel 1967 am Ende seiner Laufbahn in New Guinea an deren Beginn:

"Zu Anfang waren die meisten von uns an einsamen Stationen eingesetzt, wo wir nur unsere wenigen Bücher und unser Gewissen zu Rate ziehen konnten, wenn wir in Zweifel über den Patienten waren. Seitdem ich das Studium abgeschlossen hatte, war ich mit keinen Fällen der Geburtshilfe mehr in Berührung gekommen, (...). Am zweiten Tag nach meiner Ankunft in Mount Hagen wurde ich mit dem Problem einer seit acht Tagen zurückgehaltenen Plazenta konfrontiert, mit einer halb verfaulten Nabelschnur, die aus der Vulva hing, ein Stück verkohlten Holzes war an das Ende gebunden. (...) All die schwierigen und problematischen Entbindungsfälle wurden zu mir geschickt, besonders während der zweieinhalb Jahres meines Aufenthalts in Madang." (1967:38 u. 39)

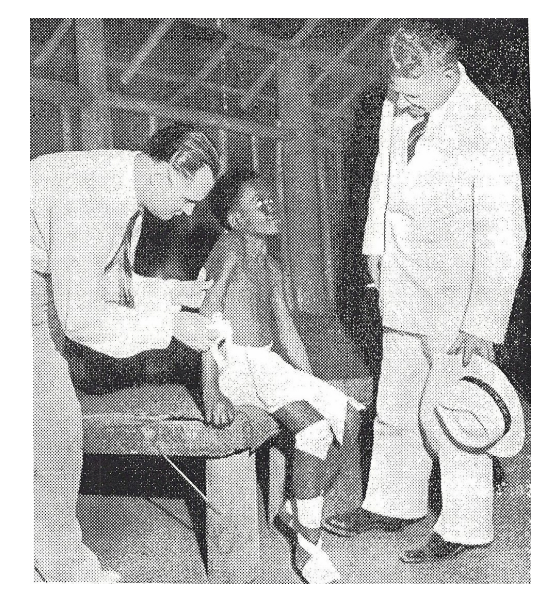

Der ungarische Arzt Dr. Francis Tuza behandelt in Anwesenheit des australoischen Direktors der Native Services, Iven Champion, ein einheimisches Kind in New Guinea. In Australien war er zuvor als Erste-Hilfe-Assistent in einem Arbeiterheim in Victoria beschäftigt gewesen. (Quelle: The Department of Immigration, The Good Neighbour, Monthly Bulletin, No. 1, August 1950) 
Für sich neu erschließen mußten die New Australian Doctors Aufgaben in administrativen und verwaltungsmedizinischen Bereichen. Zwischen 1950 und 1970 gelang ihnen, in Zusammenarbeit mit den australischen Vorgesetzten, der Aufbau einer verbesserten Gesundheitsversorgung in Papua and New Guinea. Dieser setzte den Provisorien der Vorkriegszeit ein Ende. "In den Anfangsphasen unserer Arbeit hier hatten wir kein oder nur wenig ausgebildetes oder halbausgebildetes Krankenpflegepersonal in den Hospitals. Dieser Tatbestand stellte eine große Schwierigkeit dar und brachte eine Menge Frustrationen mit sich." (Haszler 1967:39; zum folgenden 40) Das von den osteuropäischen Medizinern mitgetragene Modernisierungsprogramm umfaßte eine Vielzahl von Maßnahmen. Erwähnt seien hier auswahlweise der Aufbau eines Netzwerks von dauerhaft verfügbaren Fachärzten, auf dessen Grundlage ein großer Teil der Bevölkerung in Papua and New Guinea eine medizinische Basisversorgung erhielt. Der Start einer Anti-Tuberkulose-Kampagne; weitreichende Impfprogramme; Prophylaxe und Therapie von Yaws-, Lepra-, Malaria- und KuruErkrankungen; der Aufbau einer Geburtshilfe sowie Ansätze einer Versorgung in den Bereichen der Säuglings- und Kindermedizin stellten konkrete Ansätze dar. Weitere bedeutende Bemühungen galten der Verbesserung der medizinischen Schulung von einheimischem Assistenz- und Pflegepersonal, dem Ausbau der Präventivmedizin sowie der grundlegenden Aufklärung einer Bevölkerung, die der westlichen Kultur auch in der zweiten Hälfte des 20. Jahrhunderts noch mit Vorbehalten gegenüberstand. Der Aufbau moderner Ambulanzen und Krankenstationen fiel an das Ende der Wirkungszeit der meisten New Australian Doctors. Das ihnen auferlegte Provisorium war Ausgangspunkt für eine Entwicklung, in der die Erfahrungswerte der zugewanderten Regierungsärzte genutzt wurden, um die medizinische Infrastruktur im Verwaltungsterritorium zu vervollkommnen. Im Gefolge der Ärzte kamen weitere Fachkräfte: Apotheker, Apothekengehilfen, medizinische Hilfskräfte, Schreibpersonal. Viele von diesen Pionieren wanderten ebenfalls aus der Gemeinschaft der New Australians zu und verstärkten das Team im Department of Public Health, Papua and New Guinea.

Im Verlauf der Jahre ließ sich eine maßvolle Fluktuation im Ärzteteam beobachten. Im Jahr 1967 arbeiteten noch acht der 35 ursprünglich rekrutierten DP-Ärzte in New Guinea. Insgesamt befanden sich dort 196722 New Australian Doctors im Einsatz. Nach nationaler Zugehörigkeit ergab sich zu diesem Zeitpunkt folgendes Bild (fett gesetzt die Anzahl der aus der ersten Auswahlgruppe verbliebenen Ärzte): Ungarn (9) (4), ehemalige baltische Staatsangehörige (2) (1), Polen (3) (2), CSSR (3) (1), Niederlande (3), Österreich (1), Deutschland (1). (Haszler 1967:40). Manche hatten das Territorium verlassen, weil sich ihre Familien nicht auf Dauer an die unzureichenden Lebensverhältnisse in den Tropen zu gewöhnen vermochten, andere hofften sich in Australien fachlich weiterbilden zu können. Zwei Ärzte der ersten Gruppe verstarben, ein weiterer ungarischer Mediziner kam mit seiner Familie beim Vulkanausbruch des Higaturu ums Leben. Von den verbliebenen Kräften übernahmen im Verlauf der sechziger Jahre einige höhere administrative Verantwortung. Der ungarische Chirurg Charles Haszler entschied sich 1956, als es in New South Wales als Ergebnis einer Gesetzesänderung möglich wurde, sich in diesem Staat als ausländischer Arzt niederzulassen, für einen Verbleib in Papua and New Guinea. Er avancierte während seiner Laufbahn zum First Assistant Director of the Department of Public Health und wurde 1967 nach siebzehnjähriger Tätigkeit pensioniert. (Wigley 1967:41-42)

Aus der Rückschau stellte die Beschäftigung der New Australian Doctors in der Zivilverwaltung des Treuhandgebiets ein gelungenes Experiment dar. Als die australische Regierung 1946 die zivile Administration des Territoriums übernahm, befanden sich noch rund zwölf australische Ärzte, zum Teil aus Vorkriegszeiten, im Gebiet Papua and New Guinea. (Scragg 1964:29-30) Erst 1954, nachdem die australische Regierung mit der Entsendung der osteuropäischen Ärzte den Bestand an medizinischen Fachkräften grundlegend erweitert hatte, erfolgte erstmals wieder eine Anwerbung australischer Hochschulabsolventen. Mit der Ankunft der Ärzte aus Ungarn, den baltischen Ländern, Polen, der CSSR und Jugoslawien erweiterte sich das Spektrum der Fachdisziplinen, die in Papua and New Guinea zum Einsatz kamen. Das Hochschulstudium und die klinische Weiterbildung dieser erfahrenen Ärzte, die zum Teil auch in den Wirren des Zweiten Weltkriegs ihre Tätigkeit ausgeübt hatten, war traditionell geprägt gewesen durch eine Lehre mit deutschen 
Ausbildungskatalogen. Zwar wurde in Papua and New Guinea generell der Basisversorgung größere Bedeutung als dem Spezialistentum beigemessen, doch trug das Fachwissen der Neuankömmlinge entscheidend $\mathrm{zu}$ einer verbesserten medizinischen Ausbildung des einheimischen Pflegepersonals bei. Der Aufbau des Papuan Medical College muß als bedeutendes Nebenprodukt dieser Entwicklungsarbeit angesehen werden. (Scragg 1964:30-31 sowie 41, Schaubild IV)

Die Rekrutierung europäischer Einwanderer für den australischen Arbeitsmarkt beinhaltet ein wichtiges Kapitel in der Nachkriegsgeschichte Australiens. Umfassend und kontrovers diskutieren vor allem Historiker, Politologen, Soziologen und Sprachwissenschaftler die positiven Folgen der "multi-ethnischen" Massenzuwanderung "neuer" Volksgruppen nach 1945. Nicht immer enthält diese "Erfolgsstory" Hinweise auf Problemfälle, die auch Bestandteil der Zuwanderungsgeschichte Australiens sind. Wie das Beispiel der osteuropäischen Flüchtlingsärzte verdeutlicht, erwies sich in der Rückschau insbesondere die Anwerbung hochqualifizierter Berufsgruppen als äußerst problematisch. Hier veranschaulichte sich jener Fall, bei dem eine Regierung ausländische Talente aus bestimmten Berufsgruppen übereilt an sich $\mathrm{zu}$ binden suchte, um sie zu einem späteren Zeitpunkt für Maßnahmen der nationalen Entwicklungs- und Wirtschaftspolitik verfügbar zu haben. Der für dieses Vorgehen notwendige gesetzliche Rahmen war jedoch nicht geschaffen worden. Es gab keine verbindliche Sondergesetzgebung und damit auch keine klaren Handlungsanweisungen für eine Anerkennung der europäischen Diplome. Eine adäquate Beschäftigung der angeworbenen Akademiker konnte somit nicht gewährleistet werden. Für die Planungen der Regierung unkalkulierbar blieb das Verhalten der berufsständischen Organisationen in Australien. Deren Abschottung gegenüber ihren ausländischen Berufskollegen erwies sich gleich zu Beginn der Initiative als wirkungsvoller Gegner der "Modernisierungstendenzen" einer in Einwanderungsfragen aufgeschlossenen Labor-Regierung. An diesem Tatbestand änderte sich auch nach Ende der ChifleyRegierung (1945-49) nichts. So verlangte insbesondere der massive Widerstand der British Medical Association in Australia den um Anerkennung kämpfenden Flüchtlingsärzten einen hohen Grad der Bereitschaft zu beruflicher Neuorientierung ab. Die Anwerbung der DP-Ärzte in Australien beleuchtet aus historischer Perspektive eine einwanderungspolitische Strategie, die ihre Entsprechungen im globalen und zeitgeschichtlichen Vergleich hatte. Angesichts des nachweisbaren Erfolgs des New Guinea-Projekts bleibt kritisch zu hinterfragen, warum sich nach 1950 in Canberra keine politische Mehrheit fand, um Alternativstrategien durchzusetzen, mit denen das Fachwissen der in Australien verbliebenen Ärzte hätte genutzt werden können. Der Soziologe Egon F. Kunz bemerkte dazu in seiner Untersuchung aus dem Jahr 1975: "Man fragt sich, wie erfolgreich (wohl) ein medizinisches Projekt für Aboriginals hätte sein können, wenn den (anderen) Ärztekollegen die gleiche Chance weiter südlich in dem weiten braunen Land gegeben worden wäre." (Kunz 1975:59) Letztlich bot der New Guinea-Plan nur wenigen pionierwilligen Heimatsuchenden eine neue Existenz, ohne daß sich diese dem Zwang einer langwierigen und kostspieligen akademischen Requalifikation aussetzen mußten: "So gab es für sie nur (folgende Alternative): entweder das unbekannte New Guinea oder die Fabriken in Australien." (Haszler 1967:37) Einige von ihnen, denen der Aufbau einer beruflichen und privaten Existenz in dem von Canberra verwalteten Papua and New Guinea gelang, durchliefen einen Eingliederungs- und Anpassungsprozeß, der sie fernab der neuen Heimat, in einem australischen Außenposten, zu New Australian New Guineans machte: "Wenn ich mich an jene Tage erinnere, denke ich, daß mich nur mein Entschluß, unter allen Umständen und Bedingungen als Arzt zu arbeiten, in diesem Land hielt." (Haszler 1967:39) Eine kleine Gruppe blieb bis zum Ende der sechziger Jahre - in Papua and New Guinea.

\section{Archivalien}

Calwell, Arthur A., 1947. Conference on displaced persons, 18.7.1947. National Archives of Australia (ACT), CRS A 6980 T1, 250104 Pt.1.

Department of Immigration, The Good Neighbour. Monthly Bulletin, No.1, August 1950.

Foreign Immigration. Revised Immigration Policy-1947, 29.4. 1947. National Archives of Australia (ACT), CRS A 445/1, 235/1/2.

IRO Professional Medical Register, Geneva Headquarters, Health Division, 1948. 
Ottawa Journal, The, 8.1. 1948 .

Sydney Morning Herald, The, 1.2. 1956.

\section{Literatur}

Djonovich, Dusan J., Hsg., 1973. United Nations Resolutions. Series I-Resolutions Adopted by the General Assembly, Vol. 1: 1946-1948. New York: Oceana Publications. 84-108.

Grosart, Ian, 1973. Australian School of Pacific Administration (ASOPA), in: Peter, Ryan, Hsg., Encyclopaedia of Papua and New Guinea, Vol.1. Melbourne: University Press. 50-52.

Haszler, Charles, 1967. The New Australian Doctors in New Guinea, Papua and New Guinea Medical Journal 10(2), 35-41.

Holleuffer, Henriette von, 2001. Zwischen Fremde und Fremde. Displaced Persons in Australien, den USA und Kanada 1946-1952 (= Studien zur Historischen Migrationsforschung 9). Osnabrück: Universitätsverlag Rasch.

Krupinski, Jerzy, Alan Stoller, Lesley Wallace, 1973. Psychiatric Disorders in East European Refugees Now in Australia, Social Science \& Medicine 7(1), 31-49.

Kulischer, Alexander, Eugen M. Kulischer, 1932. Kriegs- und Wanderzüge. Weltgeschichte als Völkerbewegung. Berlin: Walter de Gruyter.

Kunz, Egon F., 1975. The Intruders. Refugee Doctors in Australia. Canberra: Australian National University Press.

Scragg, R.F.R., 1964. Presidential Address. The Medical Profession in Papua and New Guinea, 1884 to 1984, in: Papua and New Guinea Scientific Society, Hsg., Annual Report and Proceedings, Vol 16. Port Moresby: o.V., S. 22-43.

Symes, W.D., 1973. Papuan Medical College, in: Peter, Ryan, Hsg., Encyclopaedia of Papua and New Guinea, Vol. 2. Melbourne: University Press, S. 885-890.

Wigley, S.C., 1967. Dr. Charles Haszler. An Appreciation, Papua and New Guinea Medical Journal 10 (2), S. 41-42. 


\section{"Objects or Subjects?" Australienwissenschaften zwischen empirischer Ethnologie und musealer Praxis}

\section{Corinna Erckenbrecht, Köln}

\section{Einleitung}

Die klassische Definition der wissenschaftlichen Disziplin Ethnologie ${ }^{1}$ besagt, daß dieses Fach die Erforschung außereuropäischer, schriftloser, lebender Völker und Kulturen zum Ziel hat. Das heißt konkret, daß Ureinwohnervölker wie zum Beispiel die Hopi und Navajo in Nordamerika - um nur einige zu nennen -, die Yoruba oder Ashante in Westafrika, die Inuit (Eskimo) im zirkumpolaren Raum, die polynesischen, mikronesischen und melanesischen Ethnien sowie, in Australien, die Aborigines und Torres Strait Islanders ${ }^{2}$ Gegenstand der ethnologischen Forschung sind.

Diese klassische Definition ist in der Vergangenheit - zumindest im deutschsprachigen Raum auch aus ganz pragmatischen, wissenschaftsgeschichtlichen Gründen entstanden, da untergegangene, heute nicht mehr lebende Völker und Kulturen Untersuchungsgegenstand der Archäologie waren und sind und Kulturen mit eigener Schriftentwicklung demographisch und kulturell über eine solch starke Ausprägung verfügen, daß sich einzelne Wissenschaftszweige jeweils ausschließlich mit ihnen beschäftigen (wie zum Beispiel die Orientalistik, die Indologie oder die Islamwissenschaften). Die Ethnologie ist daher, wenn man so will, eine Sammelbezeichnung für die Erforschung von Gesellschaften, die die indigene Bevölkerung der jeweiligen Kontinente und Inselgebiete darstellen sowie zur Zeit ihrer "Entdeckung" keine eigene Schrift entwickelt hatten.

Moderne Kritiker dieser klassischen Definition haben dagegen eingewandt, daß in der Ethnologie Forschungstechniken und Kulturtheorien erlernt und angewendet werden, die mit gleicher Berechtigung auch auf unsere eigene Kultur und auf die Kulturen innerhalb Europas angewandt werden könn(t)en. Auch verfügen ehemals schriftlose Kulturen heutzutage in der Regel über eine Schrift - in Australien und Mittelamerika ebenso wie in Sibirien und Ozeanien - , so daß eines der drei klassischen Kriterien gar nicht mehr im ursprünglichen Sinne erfüllt ist. Dennoch sind dies ohne Zweifel nach wie vor Gesellschaften, die in das Interessens- und Forschungsgebiet der Ethnologie fallen. Bezeichnenderweise entstanden in der jüngeren Vergangenheit kulturanthropologische Richtungen, die nicht wie ehedem ihr Augenmerk ausschließlich auf schriftlose Kulturen im außereuropäischen Raum richteten, sondern sich der hiesigen Kultur(en) annahmen und in universitäre Fächer (wie beispielsweise "Empirische Kulturwissenschaften" an der Universität Tübingen) mündeten. Diese kennen keine regionalen Festlegungen und konzentrieren sich mehr auf inhaltliche Themen und die Probleme der empirischen Forschungsmethodik.

Diese neueren Richtungen, die sich im Laufe der letzten Jahrzehnte entwickelt haben, bilden zwar immer noch die Minderheit, über ihre generelle Existenzberechtigung wird heute jedoch kaum noch ernsthaft gestritten. Dies schlägt sich auch in völkerkundlichen Museumspräsentationen nieder. So wird z. B. dem Besucher des Münchner Museums für Völkerkunde neben anderen Sinnsprüchen, die zum Nachdenken anregen sollen, immer wieder der Satz vor Augen geführt: "Völkerkunde handelt von allen Kulturen und Völkern" (Heraushebung von der Verfasserin).

Abgesehen von diesen neuen programmatischen Strömungen muß jedoch betont werden, daß es in der Regel heute nach wie vor die afrikanischen, amerikanischen, asiatischen und/oder ozeanischen Ureinwohnerbevölkerungen sind, mit denen sich die Ethnologie beschäftigt, und deren Gesellschaftsstruktur, Sprache, Religion etc. an den Universitäten gelehrt wird. Und in diese Kontinente

\footnotetext{
${ }^{1}$ Der Begriff Ethnologie ist bedeutungsgleich mit dem heute vielleicht schon etwas veralteten, obgleich immer noch offiziellen Begriff "Völkerkunde", der im folgenden, insbesondere im historischen Abriß, als Synonym verwendet wird.

${ }^{2}$ Galten zunächst lediglich die Aborigines als Urbevölkerung Australiens, so unterscheidet man heute die Ureinwohner des Kontinents und die Ureinwohner der Torres Strait Islands aufgrund vieler Kulturelemente, Traditionen und physischer Merkmale voneinander. Sie stellen zwei unterschiedliche indigene Gruppen dar. Ein Sonderfall, der hier nicht ausreichend dargestellt werden kann, sind die Nachfahren der tasmanischen Ureinwohner.
} 
und Inselgebiete ziehen Ethnologen und Ethnologinnen aus, um dort ihre Feldforschung durchzuführen.

In Deutschland entstand die Ethnologie in enger Anlehnung an die Geographie und Afrikanistik, was aus der hiesigen Wissenschaftsgeschichte, der inhaltlichen wie personellen Verflechtung dieser beiden Fächer, den Entdeckungsfahrten und -zielen zum Zeitpunkt des Entstehens der Völkerkunde sowie aus der europäischen Geistesgeschichte heraus $\mathrm{zu}$ verstehen ist. Im angloamerikanischen Sprachraum hingegen entstand die Ethnologie mehr aus der Kulturanthropologie allgemein, der Linguistik und Archäologie, was wiederum mit der Wissenschaftsgeschichte und Forschungsmethodik dieser Fächer in diesen Ländern zu tun hat. Sie heißt ja dort auch "Anthropology"³, was einen wesentlich weiteren Rahmen spannt als der Begriff Ethnologie, und wird in "Cultural Anthropology", "Social Anthropology", "Linguistic Anthropology" u.a. unterschieden.

\section{Ethnologie zwischen Mensch und Museum}

Aus den vorangegangenen Erläuterungen zur Entstehung und Definition des Faches Ethnologie ersehen wir ohne Zweifel, daß es sich um eine Wissenschaft handelt, in deren Zentrum die Menschen stehen. Mit der zunehmenden Erforschung fremder Länder und Kontinente, bei der man zwangsläufig in Kontakt mit den dort lebenden Menschen kam, machte man sich daran, deren Kultur zu erforschen: die Wirtschaftsweise und Sozialordnung $\mathrm{zu}$ studieren, Religion und Rechtssysteme sowie Familienstrukuren und Begräbnissitten zu untersuchen und dergleichen mehr. All diese Themen und Teilbereiche, die noch weiter ergänzt werden könnten, sind heute Gegenstand der ethnologischen Forschung, Lehre und Publikationstätigkeit.

Über diese Beschäftigung mit Menschen hinaus - oder abgesehen davon, oder unabhängig davon wurden aber auch stets Objekte gesammelt, soweit diese für Reisende und Beobachter interessant und zugänglich bzw. erhältlich waren. Dadurch entstanden Sammlungsbestände, die in ihrem Umfang immer weiter zunahmen und zur Gründung von Museen führten. Abgesehen von vereinzelten "Kuriositätenkabinetten" oder Lehr- und Studiensammlungen des 17. und 18. Jahrhunderts entstanden Mitte des 19. Jahrhunderts die ersten geographischen und völkerkundlichen Sammlungen, die der Öffentlichkeit zugänglich gemacht wurden. Der eigentliche Aufschwung ausschließlich völkerkundlich orientierter Museen ist jedoch erst um die Jahrhundertwende anzusetzen. Gerade heute können wir daher sagen, daß die völkerkundlichen Museen einen runden Geburtstag feiern, denn sie sind ungefähr vor hundert Jahren entstanden (siehe unten).

Ethnologie im Spannungsfeld zwischen Mensch und Objekt oder zwischen Mensch und Museum dies sind also auch in der Praxis die Arbeitsfelder, in denen man beruflich als Ethnologe oder Ethnologin arbeitet. Entweder man ist im akademischen Bereich in Forschung und Lehre tätig, oder aber als Museumsethnologe mit den Sammlungsbeständen in den großen völkerkundlichen Museen befaßt. $^{4}$

\section{Das Rautenstrauch-Joest-Museum für Völkerkunde in Köln}

Befassen wir uns nun konkret mit einem völkerkundlichen Museum, dem Rautenstrauch-JoestMuseum für Völkerkunde der Stadt Köln, den dortigen Sammlungsbeständen speziell auch in Hinblick auf Australien, und exemplarisch mit den Problemen, die sich heute für Sammlungsbearbeitung und Ausstellungsmethodik ergeben.

Das Rautenstrauch-Joest-Museum für Völkerkunde wurde nach einer zweijährigen Bauzeit offiziell im Jahre 1906 eröffnet und ist damit das einzige Gebäude in Deutschland, das explizit zur

\footnotetext{
${ }^{3}$ Der Begriff der Kulturanthropologie geht ursprünglich auf den britischen Wissenschaftler Edward B. Tylor (18321917) zurück und versteht sich als Wissenschaft vom Menschen als Kulturwesen allgemein. Unter dem Einfluß von Franz Boas (1858-1942), dem Nestor der US-amerikanischen Ethnologie und Begründer der wissenschaftlichen "Cultural Anthropology", setzte sich dieser Begriff weiter durch.

${ }^{4}$ Darüber hinaus gibt es selbstverständlich noch viele weitere Tätigkeits- und Berufsfelder, in denen Ethnologen heute arbeiten, die aber im engeren Sinne nichts mehr mit der wissenschaftlichen Ausgangsdisziplin zu tun haben, so z. B. in der Entwicklungszusammenarbeit, die noch den größten Bezug zur Ethnologie hat, oder in der Museumspädadogik.
} 
Beherbergung einer völkerkundlichen Sammlung erbaut wurde. ${ }^{5}$ Das Gebäude am Ubierring erhielt seinen Namen aufgrund der umfangreichen ethnographischen Sammlungen von Wilhelm Joest, der auch als der "erste Kölner Völkerkundler" (zit. in Pützstück 1995:23) bezeichnet wird.

Wilhelm Joest wurde 1852 in Köln in eine begüterte protestantische Großbürgerfamilie hineingeboren. Er studierte in Bonn, Heidelberg und Berlin Naturwissenschaften und Sprachen und war bald von einer rastlosen Reiseleidenschaft gepackt. Seine ersten größeren Reisen - ab 1876 führten ihn zunächst in den Orient, nach Nord-, Mittel und Südamerika sowie nach Asien. Über diese Reisen hat er im Rahmen seiner regen Publikationstätigkeit mehrere Werke verfaßt. Außerdem immatrikulierte er sich an der Universität Leipzig, um bei den Professoren Bastian, Virchow und Kiepert $\mathrm{zu}$ promovieren. 1890 wurde ihm aufgrund seiner vielfältigen wissenschaftlichen Leistungen, die hier nicht alle Erwähnung finden können, der Professorentitel zuerkannt. 1896 brach Joest wieder zu einer großen Reise auf, und zwar diesmal zu der von ihm bis dahin noch nicht bereisten Südsee. Auf See verstarb Joest jedoch im November 1897 an Schwarzwasserfieber, einer Spätform der Malaria, und wurde auf Ureparapara, einer Insel in den Neuen Hebriden, heute Vanuatu, begraben.

Seine Schwester Adele Joest, verheiratete Rautenstrauch, war die Erbin des Nachlasses und damit auch der ethnographischen Sammlungen, die sie und ihr Ehemann 1899 offiziell der Stadt Köln vermachten. Die Ethnographika sollten unter Verweis auf das Joestsche Testament "ein würdiges Unterkommen in dem neuen ethnographischen-naturhistorischen Museum" (zit. in Pützstück 1995: 24) bekommen. Bis dahin waren die Sammlungen provisorisch in anderen Gebäuden der Stadt Köln untergebracht. Eugen Rautenstrauch verstarb bald darauf und Adele Rautenstrauch stiftete im Jahr 1900 das Grundkapital zum Bau eines Museums für Völkerkunde, das den Namen RautenstrauchJoest-Museum tragen sollte. Auch zum Gehalt des ersten Museumsdirektors, Willy Foy, trug sie zehn Jahre lang bei. Dieser wurde 1901 eingestellt und die Stadt Köln beschloß im selben Jahr die Finanzierung und den Bau des besagten Museums. Somit kann das Jahr 1901 als Gründungsjahr des Rautenstrauch-Joest-Museums angesehen werden.

\section{Ethnographische Objekte und ihre Ausstellungsfunktion}

Völkerkundliche Museen wie das Rautenstrauch-Joest-Museum in Köln machten es sich zum Ziel, Ethnographika aus ihren Sammlungsbeständen der Öffentlichkeit zu präsentieren und damit Kunde von fernen Völkern und Kulturen zu geben. Doch dabei blieb es nicht allein. Die Anordnung der Sammlungen und die Themen der Ausstellungen sollten auch ganz bestimmte Theorien, Zeitgeistströmungen und Gedankengebäude repräsentieren. Die Museen und die in ihnen realisierten Ausstellungen haben daher im Laufe der Wissenschaftsgeschichte immer wieder unterschiedliche Funktionen $\mathrm{zu}$ erfüllen gehabt. Dies in einer annähernd chronologischen Reihenfolge aufgelistet, ergibt folgendes Bild:

Es handelte sich zunächst um Studien- und Lehrsammlungen für Gelehrte und Studenten, wobei auch nur diese Zugang zu den Objekten hatten. Die Objekte sollten Kultureigentümlichkeiten zeigen, aber auch die besondere Primitivität der Völker belegen (s.u.). Durch die Art und Weise, wie Ethnographika präsentiert wurde (Objektgruppen, regionale und kulturelle Zusammenfassungen, räumliche Anordnung) sollten Kulturkreise bzw. Kulturprovinzen und deren Abfolge zusammenfassend dargestellt werden (s.u.). Die Ausstellungen sollten aufklärerisch wirken; sie sollten bilden und erziehen; sie sollten soziale und kulturelle Hintergründe aufzeigen und sozialkritisch sein; und schließlich sollten sie künstlerisch bildend wirken und ästhetischen Genuß verschaffen.

An dieser Vielzahl der Funktionen, die Museen und die in ihnen präsentierten Objekte im Laufe der Geschichte einnahmen bzw. in den Augen der Ausstellungsmacher einnehmen sollten, ersieht man die Wechselfälle der Museums- und Ausstellungspolitik. Hinzu kamen die inhaltlichen und theoretischen Strömungen des Faches Ethnologie, die sich im Laufe von 150 Jahren Wissenschafts-

\footnotetext{
${ }^{5}$ Alle Angaben zur Gründungsgeschichte des Rautenstrauch-Joest-Museums und zu den Personalia über Wilhelm Joest sind den zwei Publikationen entnommen: Pützstück (1995) und Völger (1999).
} 
geschichte immer wieder wandelten. In unserem Fall wollen wir uns speziell mit den Gedankengebäuden und Theorien beschäftigen, die zur Zeit der Gründung völkerkundlicher Museen vorherrschend waren. Sie beeinflußten nicht nur Ausstellungsmethodik und Sammlungs-wesen, sondern ließen insbesondere die Kultur der australischen Aborigines in ihre Überlegungen mit einfließen. Es handelte sich um die damals bestimmende Theorie des Evolutionismus, aber noch viel mehr um die Theorie der Kulturkreislehre, die maßgeblich von deutschen Wissenschaftlern, insbesondere aus Köln, und später von österreichischen Wissenschaftlern bzw. Geistlichen weiterentwickelt wurde. Daher spricht man auch von der "Kölner Schule" und der "Wiener Schule". Diese beiden theoretischen Richtungen, Evolutionismus und Kulturkreislehre, sollen nun speziell in Bezug auf die australischen Ureinwohner und das damalige Ausstellungswesen vorgestellt werden.

\section{Evolutionismus, Kulturkreislehre und die Aborigines}

Die schriftlichen Museumsführer durch die Schausammlungen des Rautenstrauch-Joest-Museums, die ab 1906 von dem ersten Direktor Willy Foy verfaßt wurden, insbesondere aber der "Führer durch das Rautenstrauch-Joest-Museum" in seiner 3. Auflage von 1910, wird heute von Wissenschaftshistorikern als eine "Bibel der Kulturkreislehre" (in Völger 1999:7) bezeichnet. Damit ist dies ein Paradebeispiel für die um die Jahrhundertwende und zuvor herrschende Überzeugung, die menschliche Kulturentwicklung sei weltweit in eine universalhistorische Abfolge zu bringen. Denn auch der Evolutionismus der 2. Hälfte des 19. Jahrhunderts hatte einen ähnlichen Ansatzpunkt: Man wollte alle bekannten Kulturen und Gesellschaften einem (hypothetischen) Entwicklungsraster zuordnen, auf dem sich diese wie auf einer Rangleiter von der untersten bis zur obersten Sprosse fortentwickelten. Dabei versuchte man, alle Aspekte des wirtschaftlichen, sozialen, kulturellen wie religiösen Lebens in eine Reihen- und Rangfolge einzugliedern. Als ein Beispiel dafür mag das von Lewis Henry Morgan in seinem Hauptwerk "Ancient Society" (1877) entwickelte globale Schema gelten, demzufolge sich die menschliche Gesellschaft vom Zustand der Wildheit über die Barbarei zur Zivilisation entwickelt habe. ${ }^{6}$

Um die Theorie der darauf folgenden Kulturkeislehre, in deren Zeit die Gründung des Rautenstrauch-Joest-Museums fällt, nun etwas näher $\mathrm{zu}$ beschreiben, zitiere ich aus dem Ausstellungskatalog "Kunst der Welt" (Hrsg. v. Völger 1999), der die einhundert schönsten Stücke des Hauses vorstellt, der darüber hinaus aber sowohl im Vorwort als auch im Anhang auf die Geschichte des Hauses eingeht. In diesem Zusammenhang heißt es unter anderem:

"Mit diesem Begriff [Kulturkreislehre, C.E.] bezeichnete man zu Beginn des 20. Jahrhunderts die gängige Methode zur Ordnung der Kulturen aufgrund von stilistischen und technologischen Merkmalen der in den Museen bewahrten und eigens zum Zwecke derartiger Untersuchungen gesammelten Objekte. Durch den Vergleich von Techniken und Dekorationsformen glaubte man, kulturelle Verbindungen zwischen Kulturen rekonstruieren und auf diese Weise die Geschichte der schriftlosen Völker schreiben zu können. Eine Funktionsbestimmung, die Bedeutung der Objekte innerhalb der jeweiligen Kultur oder gar ästhetische Kriterien waren vielen der damaligen Forscher kein Anliegen." (S. 7)

Die Kulturkreislehre hatte also, ähnlich wie der Evolutionismus, den Anspruch, eine univeralhistorische Kulturgeschichte der Menschheit zu schreiben, und dies unter Zuhilfenahme der Objekte. Die Völker und Kulturen, mit denen man sich beschäftigte, besaßen ja, wie wir wissen, keine eigene Schrift, keine schriftlichen Überlieferungen. Um aber eine historische Perspektive in diese Kulturen hineinzubringen, benutzte man die Objekte. Man untersuchte und verglich die Objekte einzelner Kulturen, entwickelte Kriterien, nach denen man sie klassifizieren konnte und

\footnotetext{
${ }^{6}$ Lewis Henry Morgan (1818-1881), ursprünglich Rechtsanwalt, übte erheblichen Einfluß auf die Theorienbildung innerhalb der jungen Ethnologie aus. Durch seinen universalhistorischen Ansatz, der sich an konkret belegbaren Kulturmerkmalen vor allem wirtschaftlicher Art orientierte, hatte er aber auch großen Einfluß auf den ebenfalls im Entstehen begriffenen Marxismus bzw. historischen Materialismus.
} 
versuchte so, ganze Kulturen zu Gruppen, eben sogenannten Kulturkreisen, zusammenzufassen und diese in eine zeitliche Abfolge zu bringen. ${ }^{7}$

Die theoretische Straffung und Untermauerung der Lehre von den Kulturkreisen wurde 1911 von Fritz Gräbner in seinem Buch "Die Methode der Ethnologie" geleistet. Gräbner (1877-1934), ursprünglich Historiker, entwickelte sich zu einem bedeutenden Museumsethnologen und war ab 1906 Foys Assistent in Köln, ab 1924 selbst Direktor des Rautenstrauch-Joest-Museums. Weitere Publikationen Gräbners, die sehr gut die Denkweise jener Zeit veranschaulichen können, lauteten z. B. "Kulturkreise und Kulturschichten in Ozeanien" (1905), "Wanderung und Entwicklung sozialer Systeme in Australien" (1906) oder "Die melanesische Bogenkultur und ihre Verwandten" (1909). Auch die "Wiener Schule" der Linguisten, Ethnologen und Religionswissenschaftler Wilhelm $\mathrm{Schmidt}^{8}$ und Wilhelm Koppers (u.a.), die in den '20er bis '40er Jahren des 20. Jahrhunderts eine wichtige Rolle spielte, beruhte auf der oben beschriebenen kulturhistorischen Methode. Erstmals wurden ihre Ideen in dem Werk "Völker und Kulturen" aus dem Jahr 1924 zusammengefaßt. Weitere bezeichnende Titel waren "Die Sprachfamilien und Sprachkreise der Erde" (1926), "Handbuch der Methode der kulturhistorischen Ethnologie" (1937) sowie das weltumspannende Hauptwerk Schmidts "Der Ursprung der Gottesidee" (1912-1955).

Hierin spielten auch die australischen Ureinwohner und ihre religiösen Vorstellungen eine große Rolle. Der Gelehrte und Geistliche wollte nämlich beweisen, daß es einen ursprünglichen Monotheismus bei den sogenannten Naturvölkern gegeben habe. Dieser habe sich dann abgewandelt, sei in degenerierte Formen wie den Polytheismus übergegangen, um dann zum Schluß, wie bei uns in den modernen Hochkulturen vorzufinden, wieder in einen Monotheismus zu münden. Da man in evolutionistischer Manier seinerzeit annahm, die ersten Australier befänden sich auf der untersten Stufe der Menschheitsentwicklung, suchte man auch hier nach Beweisen für den Urmonotheismus und glaubte, ihn gefunden zu haben. So zum Beispiel in dem Glauben an ein "Höchstes Wesen" bei den südostaustralischen Aborigines-Gruppen. Daß dieses "Höchste Wesen" (wie Baiame bei den Kamilaroi und benachbarten Ethnien in Südostaustralien) eher ein Kulturheros aus der autochthonen Religionsform der Traumzeit war, wurde nach damaliger Auslegung geflissentlich übersehen. ${ }^{9}$ Diese Forschungen sind daher ein gutes Lehrstück darüber, was man so alles sehen kann, wenn man es nur sehen will. Außerdem belegen sie die überaus große Bedeutung, die die australischen Aborigines in der ethnologischen Theorienbildung stets innehatten.

Doch zurück zu der Kulturkreislehre, bei der, wie erwähnt, die Objekte im Vordergrund standen. Diese brachte man in eine chronologische, universalhistorische Abfolge und richtete auch die Ausstellungseinheiten danach aus. Dabei sollten zunächst die älteren, d.h. "primitiven" und niedrig stehenden Kulturschichten vorgestellt werden, dann ging es langsam zu höher entwickelten Kulturschichten bis dann die vermeintlich höchsten folgten, die die ersteren teils überlagerten. Entsprechend diesem Glauben ordnete man nun in Völkerkundemuseen die Kulturen auch räumlich aus. Die vermeintlich "niedrigste" Kulturform, nämlich Australien, befand sich demzufolge im Keller des Hauses, und man bewegte sich dann in den drei folgenden Etagen nach oben zu den "höherentwickelten" Kulturen, die man in Indien und Indonesien verwirklicht sah, allerdings nicht ohne den Hinweis, daß in diesen Gebieten primitivere Kulturformen überlebt hätten.

Die Bewohner des fünften Kontinents charakterisierte einer der Museumsführer folgendermaßen:

\footnotetext{
${ }^{7}$ Ursprünglich hatte der bedeutende Afrikaforscher und Kulturphilosoph Leo Frobenius (1873-1938) den Begriff des Kulturkreises in die Ethnologie eingeführt. Frobenius selbst vertrat allerdings später die Lehre der Kulturmorphologie, nach der Kulturen lebende Organismen mit einer individuellen Seele seien.

${ }^{8}$ Pater Wilhelm Schmidt (1868-1954) war Priester des Missionsordens Societas Verbi Divini (S.V.D.) und gilt als der Begründer der "Wiener Schule" der Völkerkunde. Er initiierte "Anthropos", die heute noch bedeutende Internationale Zeitschrift für Völker- und Sprachenkunde, sowie das päpstliche ethnologische Museum in Rom.

${ }^{9}$ Mit der Diskussion um einen möglichen Urmonotheismus bei den südostaustralischen Aborigines-Gruppen habe ich mich ausführlich auseinandergesetzt, da sie eine Ethnie betrafen - die Kamilaroi - , bei der ich selbst meine zeitgenössische Feldforschung durchgeführt hatte (vgl. Erckenbrecht 1993a/b).
} 
"Australien ist von einer dunkelhäutigen Bevölkerung bewohnt, die sowohl in körperlicher wie in kultureller Beziehung außerordentlich primitive Züge aus der Anfangsentwicklung der Menschheit bewahrt hat, wie sie in gleichem Maße an keiner anderen Stelle der Erde sich finden. Das hat seinen guten Grund darin, daß Australien, von allen jüngeren Völkerströmen, die von Asien aus die Südsee überflutet haben, so gut wie unberührt geblieben ist. Dadurch besitzt es für die Völkerkunde sowohl wie für die physische Anthropologie einzigartige Bedeutung." (Foy 1910:51)

Erwähnt wurden des weiteren die "ganz primitiv[e] (...) nomadische Lebensweise", fast fehlende Kleidung und Musikinstrumente, die "primitiven" Waffen wie Keule, Bumerang und Parierschild sowie die Nutzung des Grabstocks als "ältestem landwirtschaftlichem Gerät" (Foy 1910:51ff.). Hier sehen wir beispielhaft das Bedürfnis jener Zeit bzw. Theorierichtung, materielle Objekte in ein perspektivisches, historisches Gefüge einzuordnen und sie in "alt" und "jung" zu klassifizieren. Auch auf die Religion, die man als Zauberglaube und primitive Form des Seelenglaubens interpretierte, wurde eingegangen. ${ }^{10}$ Dabei repetiert Foy interessanterweise auch jene Idee des Urmonotheismus, die damals im Schwange war:

"Zunächst überrraschend wirkt es, namentlich in Südostaustralien den Glauben an einen Gott, den 'Vater' oder 'Großvater' zu finden, der als Schöpfer des Menschen und der wichtigsten Naturerscheinungen gilt, der die Menschen die Herstellung der Waffen und Geräte gelehrt hat und über die Innehaltung der Stammesgesetze, besonders über die richtige Ausführung der komplizier-ten Jünglingsweihen wacht." (Foy 1910:58f.)

Hier schließt sich wieder der Kreis, den die Wiener Ethnologen und Geistlichen mit ihren Forschungen zur Religion zur Vollendung bringen wollten. In den australischen Ureinwohnern sah man um die Jahrhundertwende und noch zwanzig bis dreißig Jahre danach die unterste, und damit niedrigste bzw. primitivste Stufe der menschlichen Kulturentwicklung, was die einen, die Kulturkreisler, hauptsächlich an den Objekten, die anderen, die Wiener Schule, hauptsächlich an der Religionsform festmachten. Eine induktive Erforschung der Kultur, die nach keiner vorgefertigten Theorie vorging und die Gesellschaften ahistorisch in ihren Grundfunktionen und -institutionen erforschen wollte, begann erst nach Überwindung des historischen Ansatzes mit den britischen bzw. französischen Schulen des Funktionalismus und Strukturalismus.

Wenden wir uns nun noch kurz der Australiensammlung des Kölner Museums zu, um danach die Entwicklung von Theorien, Forschungsmethoden und Museumsausstellungen in einem größeren Überblick Revue passieren zu lassen.

\section{Hermann Klaatsch und die Kölner Australiensammlung}

Über Umfang, Zustandekommen und Charakteristika der Kölner Australiensammlung habe ich bereits im letzten Newsletter einige Zeilen geschrieben ("Die materielle Kultur der australischen Aborigines - eine 'Stein'zeit?" Newsletter 14, 27-42). Die Sammlung geht im wesentlichen auf das Konvolut von 862 Objekten zurück, die am 11. März 1908 von Hermann Klaatsch erworben wurden. Klaatsch (1863-1916), ein physischer Anthropologe und Hobbyethnologe, hatte sich von 1904 bis $1907 \mathrm{zu}$ ausgedehnten Reisen in Australien aufgehalten und insgesamt über 2000 Ethnographika erworben, die er später an die Museen in Köln, Hamburg und Leipzig verkaufte.

Klaatsch war ganz Kind seiner Zeit, der in den australischen Ureinwohnern ein "missing link" zu den Vorfahren aus der Steinzeit zu erkennen glaubte und die ersten Australier auf der untersten Stufe der menschlichen Entwicklungsreihe ansiedelte. Entsprechend paternalistisch und respektlos war sein Verhalten den Ureinwohnern gegenüber, wenn er an Objekte, aber auch an die seinerzeit begehrten Skelette und Schädel herankommen wollte. Während seine Hauptzuträger, Informanten und Unterstützer auf seiner Reise rund um Australien Missionare und sogenannte "Protektoren" der Aborigines waren, so suchte er, wenn er sich in Siedlungen der Weißen befand, in erster Linie

\footnotetext{
${ }^{10}$ Die Geistkindvorstellung wird allerdings schon recht treffend wie folgt wiedergegeben: "Die neugeborenen Kinder gelten entweder nur als Wiedergeburt von Ahnen oder werden von bestimmten Bäumen, Felsen oder Teichen abgeleitet, die man als Sitze ungeborener Kinder betrachtet." (Foy 1910:57)
} 
Krankenhäuser und Gefängnisse auf, denn das waren in diesem Umfeld die einzigen Orte, an denen Ureinwohner zu finden waren. Auch Friedhöfe erregten sein Interesse, da er hier an Skelette und Schädel herankommen konnte, auch wenn er dafür große Gefahren in Kauf nehmen mußte, wie ihm recht wohl bewußt war. So schrieb er nach dem Raub von Skelettteilen eines "jungen, weiblichen Individuums, das während meiner Anwesenheit starb", recht realistisch: "Hätten die Schwarzen den Raub entdeckt - die Folgen hätten verhängnisvoll werden können für die Missionare und für mich." (Dies und das folgende Zitat stammen aus Völger 1986:254.) Als er - diesmal auf Melville Island in Nordaustralien - vom Tod eines weißen Begleiters und Führers erfuhr, vermutete er sofort das Naheliegende: "[Es] ist zu befürchten, daß Cooper ein Opfer der Rache für die Verletzung der Gräber geworden ist."

Nichtsdestotrotz machte sich Klaatsch mit der nötigen Unverfrorenheit und Überheblichkeit an seine Arbeit, wie die folgenden Zitate belegen. In einen Brief an Georg Thilenius, den Professor und Direktor des Hamburger Museums für Völkerkunde und wesentlichen Finanzierer seiner Reisen, schrieb er z. B. in einem Brief vom 12.-14.11.1905: "Ich habe sehr zahlreiche Photographien genommen; nur nackte Weiber sind stets schwierig für die Photographie zu haben, selbst bei den ganz Wilden." (zit. in Völger 1986:249).

Auch über seine anatomischen Messungen, die er an den Aborigines vornahm, äußerte er sich in der typischen Manier eines germanischen Schädelvermessers: "Für meine somatischen Studien erwies sich ein Besuch der Missionsstation in Cape Bedford nördlich von Cooktown sehr nützlich. Ich fand gut gewaschenes und geduldiges Menschenmaterial, um meine Messungen fortzusetzen." (zit. in Völger 1986:254)

Klaatschs Ansichten waren sicherlich typisch für jene vom Evolutionismus geprägte Zeit, in der man die australischen Aborigines für eine der am niedrigsten stehenden Kulturen hielt, die früher oder später vom Aussterben bedroht sein würden. Auch Klaatsch verstand daher seine Sammelreisen als eine Art "Rettungswerk", um so mehr, da sich deutsche Museen an den Zeugnissen dieser Kultur sehr interessiert zeigten. Bereits am 7. September 1907 eröffnete das Rautenstrauch-Joest-Museum seine erste Sonderausstellung über die australischen Aborigines, wobei 2666 Objekte von Klaatsch, der erst am 3. April desselben Jahres aus Australien zurückgekehrt war, gezeigt wurden.

Neben den Beständen aus Klaatsch's Sammeltätigkeit setzt sich die übrige Kölner Australiensammlung aus Gaben vieler verschiedener Spender und Stifter zusammen, die hier nicht im Einzelnen genannt werden können. Besonders erwähnt werden muß bei all diesen Sammlungstätigkeiten und Eingängen in völkerkundliche Museen - und das gilt nicht nur für Köln, sondern für alle Völkerkundemuseen jener Zeit -, daß nicht repräsentativ, sondern selektiv und zufallsbedingt gesammelt wurde. Das, was gerade interessierte oder erhältlich war, wurde vor Ort gekauft oder eingetauscht. An die Erstellung eines repräsentativen Querschnitts der materiellen Kultur wurde dabei nicht gedacht. So kommt es, daß in Australiensammlungen europäischer Museen überdurchschnittlich viele Waffen und Werkzeuge wie z. B. Bumerangs, Speere und Schilde vertreten sind, wohingegen Objekte aus anderen Wirtschaftszweigen wie dem Sammeln und dem Fischfang oder auch aus Bereichen wie dem Körperschmuck, der Dekoration und der Musik etc. völlig fehlen. ${ }^{11}$

\section{Entwicklungsraster Ethnologie}

Eine tabellarische Auflistung, die die Entwicklung des Faches Ethnologie und seiner Theoriegebäude sowie die Entstehung der Völkerkundemuseen mit ihren Ausstellungsmethoden darstellt, folgt auf den nächsten beiden Seiten. Dazu muß angemerkt werden, daß eine solche Darstellung aus Platzgründen verkürzen und komprimieren muß, denn nicht auf alle Teilbereiche und wissenschaftliche Strömungen kann dabei im einzelnen eingegangen werden. Dennoch bietet

\footnotetext{
11 Auch eine geschlechtsspezifische Analyse der Ankaufs- und Erwerbsgeschichte von Völkerkundemuseen könnte interessant sein. Da die Museumsleiter zumeist Männer waren, wurden hauptsächlich Waffen und Werkzeuge angeschafft. Wären es Frauen gewesen, hätten Grabstöcke und Sammelschalen Vorrang gehabt?
} 
eine solche Übersicht, wie ich glaube, einen gut geeigneten Einstieg in Geschichte und Entwicklung der Völkerkunde sowie ihrer Museen.

\section{Bedeutung für Australiensammlungen in Völkerkundemuseen}

Aus dem Entwicklungsraster lassen sich bestimmte Schlüsse für die heutige Zusammensetzung von völkerkundlichen Sammlungsbeständen aus Australien, aber auch aus anderen Regionen der Welt ziehen. Zum einen müssen wir uns eingestehen, daß wir es in fast allen Fällen mit historisch abgeschlossenen Beständen $\mathrm{zu}$ tun haben, die uns nur ein kleines zeitliches Fenster auf die materielle Kultur der betreffenden Gesellschaft(en) eröffnen. Diese Bestände sind in punkto geographischer Verteilung und Objektauswahl eher zufällig entstanden, und zwar jeweils dort, wo Reisende die Möglichkeit hatten hinzugelangen, wo Einhandlungsmöglichkeiten bestanden oder wo z. B. Mis-sionsstationen existierten. Selten wurden australische Objekte direkt von den Aborigines erstanden, sondern meistens über Dritte erworben; hier spielten auch die zunehmend professionell und kommerziell agierenden Ethnographikahändler eine Rolle. Sie belieferten u. a. jene deutschen Völkerkundemuseen, die sich darum bemühten, ihre oftmals einseitigen Sammlungsbestände regional und/oder typologisch zu erweitern.

Wichtig für ethnographische Sammlungen aus Australien waren aber besonders solche Missionsstationen bzw. Missionare, die lange vor Ort tätig waren und ein Vertrauensverhältnis zu den örtlichen Aborigines und ihren Ältesten entwickeln konnten, wie z. B. der deutsche Missionar bei den westlichen Aranda, Pastor Carl Strehlow. Durch die Größe des australischen Kontinents und sein unwegsames Inneres verfügen wir heute hauptsächlich über Objektbestände von den Küstenrändern oder den Randgebieten der damaligen "frontier-society". Die abgelegenen Inlandsgebiete sind hingegen nur punktuell vertreten, nämlich dort, wo, wie erwähnt, Missionsstationen existierten, oder wo große Farmen bestanden, die Reisende anlaufen konnten. Aber selbst hier wurden unter Umständen nur bunt gemischte Objektbestände erworben, da Missionsstationen einen riesigen Einzugsbereich hatten bzw. auch Aborigines aus weit entfernten Gebieten zwangsweise dorthin umgesiedelt worden sein konnten. Auch auf den Farmen der Weißen lebten mitunter ganz unterschiedliche Stammesgruppen zusammen, die aufgrund vieler Wechselfälle dort angesiedelt worden waren oder zufällig Arbeit und Verpflegung erhalten hatten. Aus den so erworbenen Objektbeständen konnten daher nur relativ grobe regionale Zuordnungen und oftmals keine ethnischen Zuordnungen rekonstruiert werden.

Besondere Erwähnung in Zusammenhang mit Australien müssen jene Sammlungsbestandteile finden, die ein trübes Kapitel des Kulturkontakts und der Erwerbsgeschichte von Museen beleuchten: Durch den damaligen Zeitgeist und die evolutionistische Überzeugung, bei den australischen Aborigines ein "missing link" zu den Anfängen der Menschheit zu finden, wurde besonders Jagd - im wahrsten Sinne des Wortes! - auf menschliche Skelette gemacht, was heute zu problematischen Objektbestandteilen in europäischen Museen führt, den sogenannten "human remains". 12

\section{Heutige Probleme der Sammlungsbestände und der musealen Arbeit}

Aus diesen und weiteren Faktoren ergeben sich heute spezifische Probleme der Sammlungszusammensetzungen und der musealen Arbeit, die, so meine Thesen, zu ganz bestimmten Konsequenzen führen müssen. Im folgenden erläutere ich nun diese Problemfelder und leite dann im letzten Punkt zu den von mir geforderten Konsequenzen über. Dabei spitze ich bewußt einige Thesen zu, um Mißstände, Defizite oder mögliche Lösungen klarer hervortreten zu lassen.

\footnotetext{
${ }^{12}$ Hierbei muß allerdings erwähnt werden, daß Schädel und Knochen menschlicher (oder tierischer) Herkunft zum "normalen" Bestandteil völkerkundlicher Sammlungen gehören können. In den betreffenden Kulturen war der Tod nicht derart tabuisiert wie bei uns. Es existierten Schädel- oder Ahnenkulte, es gab Sekundärbestattungen oder rituelle Anthropophagie (Kannibalismus), also alles Verhaltensweisen, die den Umgang mit menschlichen Schädeln und Skeletten erforderte. Auf den aktuellen Stand der Rückgabeforderungen bzw. der Rückgabepolitik kann aufgrund der komplexen Problematik hier nicht ausreichend eingegangen werden.
} 


\subsection{Zeitliche und geographische Diskontinuität}

Aufgrund von historischen und forschungsbedingten Hintergründen, die oben geschildert wurden, haben wir es heute mit (australischen) Ethnographika zu tun, die eine zeitliche und geographische Diskontinuität aufweisen. Es handelt sich dabei um ein "Zeitfenster", das in etwa um die vorige Jahrhundertwende anzusiedeln ist. Ethnographische Sammlungen australischer materieller Kultur wurden in der zweiten Hälfte des 19. Jahrhunderts begonnen, als noch ein kontinuierlicher Kontakt $\mathrm{zu}$ Aborigines-Gruppen bestand, die ihr traditionelles Leben aufrechterhalten oder zumindest in Ansätzen praktizieren konnten. Zu Beginn des 20. Jahrhunderts und endgültig in den '20er und '30er Jahren versiegten dann die Möglichkeiten und das Interesse, Ethnographika zu erwerben, da die Aborigines nun auf Reservats- oder Missionsstationen lebten und ihre ehemals ungebundene Lebensweise aufgegeben hatten bzw. hatten aufgeben müssen. Von ihrem Land, das ihnen eine wirtschaftliche Subsistenzsicherung geboten hatte, waren sie größtenteils schon vertrieben worden. Nur in wenigen Enklaven in Nord- und Zentralaustralien konnte sich ein unbeeinträchtigter "aboriginal way of life" noch erhalten.

Ethnographikasammler, aber auch Wissenschaftler (und Missionare), die ethnologisches Wissen erwerben wollten, sahen sich seinerzeit getrieben von dem Geist, "noch zu retten, was zu retten ist." Sie verstanden sich als Bewahrer und "Rekonstrukteure", prangerten den Kulturwandel und seine schädlichen Einflüsse an und wollten das möglichst Authentische aus der vorgefundenen Kultur bewahren oder wieder heraus"schälen". Ihre Arbeit verstanden sie als eine Art Rettungswerk, das unter enormem Zeitdruck stand.

Um die Jahrhundertwende glaubte man zudem, die Aborigines würden endgültig aussterben. Ihre Bevölkerungszahlen sanken und Krankheiten, geringe Lebenserwartung, niedrige Geburtenrate sowie mangelnde Integration in die weiße Gesellschaft schienen das Ende der ersten Australier zu besiegeln. Die Parole der weißen Gesellschaft lautete daher: "To smooth the dying pillow." Das Sterbekissen sollte schön sanft gestaltet sein. Daher bemühte man sich, solange die Ureinwohner des fünften Kontinents noch lebten, ihre soziale und materielle Kultur zu dokumentieren.

Dieser Dokumentationswille von scheinbar dem Untergang Geweihten füllt heute unsere Museumsdepots, so meine These. Wir wissen jedoch kaum etwas bis gar nichts darüber, wie die Aborigines überlebten - und daß dem so ist, diese Tatsache steht heute fest. ${ }^{13}$ Welche Objekte, welche materiellen Güter besaßen und benutzten sie, um in der Welt der Weißen zu überleben, welche Häuser, Wohnungen, Möbel, Wellblechhütten und Wolldecken, oder welche Grabstöcke oder Speere benutzten sie in den Jahren des Umbruchs, des indigenen Kulturverlusts? Welche Objekte der europäischen Welt nutzten sie oder fertigten sie um, welche lehnten sie ab? Da wir darüber keine Aufzeichnungen, keine Dokumente und keine Objekte besitzen, sind uns etliche Jahrzehnte des autochthonen Lebens verlorengegangen.

Wenn wir auch - anhand der Objekte - vermuten können, wie die australischen Aborigines vor hundert Jahren lebten und wir wissen, wie sie heute leben, das Jahrhundert dazwischen ist für uns, und dadurch oftmals auch für sie selbst, eine kaum dokumentierte Zeit.

\subsection{Verzahnung von Geschichte ( = Objekte) und Gegenwart ( = Menschen)}

Objektsammlungen in völkerkundlichen Museen sind, wie oben festgestellt, in den meisten Fällen hundert Jahre alt oder noch älter, weswegen ich sie hier einmal als "ethnographische Geschichte" bezeichnen möchte. Die ethnologische Forschung in Deutschland hat jedoch heute - im Gegensatz zu früher - keinen historischen Charakter mehr, sondern ist zunächst gegenwartsbezogen. Alleine

\footnotetext{
${ }^{13}$ Den Scheideweg in dieser Hinsicht stellten die '40er Jahre und der zweite Weltkrieg dar. Für Australien lag der Feind (Japan) im Norden, so daß Zentral- und Nordaustralien plötzlich im Mittelpunkt des nationalen Interesses standen. Nicht nur die Militärmaschinerie wurde nach Norden verlegt, auch viele zivile Einrichtungen und die Infrastruktur profitierten von dieser neuen geostrategischen Bedeutung Zentral- und Nordaustraliens. Nun kamen viele Australier, ob Soldaten oder Zivilisten, erstmals tatsächlich in Berührung mit Aborigines und deren Lebensbedingungen. Die mächtigen Gewerkschaftsverbände setzten sich für sie ein. Dieses neue Augenmerk und viele Verbesserungen vor allem in der Gesundheitspolitik bewahrten die australischen Ureinwohner letztlich vor dem Aussterben. Vergessen werden darf hier aber auch nicht der von den Staatsorganen so nicht erwartete rapide Anstieg der Mischlingsbevölkerung.
} 
die Tatsache, daß die stationäre Feldforschung mit teilnehmender Beobachtung als "Königsweg" der empirischen Datenerhebung gilt, macht deutlich, daß sich die ethnologische Forschung mit den Menschen von heute beschäftigt. Andererseits wird auch hier immer wieder quasi durch die Hintertür versucht, eine historische Perspektive hineinzubringen, wie die weiteren kritischen Ausführungen zeigen werden (siehe insbesondere Punkt 9.7). Hier klafft eine große Lücke: Die in der Feldforschung gewonnenen Daten zur sozialen Kultur (= Gegenwart) lassen sich oftmals mit der in den Museen bewahrten materiellen Kultur (= Vergangenheit) nicht in Einklang bringen. Hier fehlen uns, wie schon gesagt, de facto hundert Jahre, über die wir so gut wie nichts wissen.

Selbstverständlich gibt es heutzutage auch Bemühungen, die gerade in diesem Zwischenbereich ansetzen und die geschilderte Lücke schließen wollen. Dennoch bleibt m. E. ganz grundsätzlich die Tendenz bestehen, daß - heute mehr denn je - ein Zwiespalt zwischen historischen Sammlungsbeständen und gegenwartsbezogener Forschung herrscht.

\subsection{Das Problem der religiösen Artefakte}

Religiöses Wissen in Australien war geheim. Es stellte einen nicht-öffentlichen Sektor des sakralen Lebens dar und drückte sich auch in der Geheimhaltung religiöser Artefakte aus. Diese wurden in der Regel nur Eingeweihten enthüllt, die auch in die religiöse oder mythische Bedeutung des Objekts sowie in seinen Kontext in den Überlieferungen eingeweiht wurden.

Sammlungsleidenschaft und Respektlosigkeit einerseits sowie Unkenntnis der australischen Religion andererseits brachten viele dieser Sakralobjekte in europäische Museen, wo sie zunächst auch bedenkenlos ausgestellt wurden. Erst in jüngerer Zeit hat sich die Überzeugung durchgesetzt, solche Sakralobjekte der Öffentlichkeit nicht mehr zu präsentieren. Das bedeutet, daß heute eine beträcht-liche Anzahl religiöser heilig-geheimer Objekte in europäischen Museumsdepots schlummern. Aber auch Depots müssen umgeräumt, Objekte gesäubert werden, so daß eine vollständige Seklusion praktisch unmöglich ist. Die öffentliche Präsentation, die zwar heutepolitisch korrekt—nicht mehr vorgenommen wird, ist eine Sache, die praktische Arbeit mit den Objekten in den Depots eine andere.

\subsection{Der Zustand der Objekte}

Das Alter der Sammlungsbestände und der Auftrag der Museen, diese Kulturgüter zu wahren, schließt eine sorgfältige Aufbewahrung und Restaurierung mit ein. Angesichts leerer Kassen können diese notwendigen Arbeiten jedoch oftmals kaum bis gar nicht gewährleistet werden. Auch die baulichen Voraussetzungen der zumeist alten Gebäude können nicht immer die sach- und fachgerechte Lagerung ermöglichen. Hinzu kommt die immens hohe Anzahl an Objekten. Ein Haus wie das Völkerkundemuseum in Köln beherbergt z. B. insgesamt ca. 55000 ethnographische Objekte, in anderen Museen sind es zum Teil noch wesentlich mehr. All diese Ethnographika über Jahre und Jahrzehnte, ja sogar Jahrhunderte, stets einwandfrei zu erhalten, ist eine Mammutaufgabe, die mit dem Alter der Objekte größer wird und die oftmals an den Gegebenheiten des Alltags scheitert.

\subsection{Ausstellungs"ideologien"}

Wie zu Beginn meiner Ausführungen und aus dem "Entwicklungsraster Ethnologie" ersichtlich, hat es in der Vergangenheit immer wieder verschiedene Methoden und auch Moden gegeben, Ethnographika zu präsentieren. Wenn wir an dieser Stelle einmal auf die jüngeren Beispiele eingehen, so können wir eine sozialkritische, kontextuale Phase in den '70er und '80er Jahren und eine künstlerisch-puristische, manchmal auch als postmodern bezeichnete Phase in den '90er Jahren ausmachen. Bei ersterer sollten die Lebensbedingungen der Völker und Kulturen aufgezeigt und die Besucher für die Probleme in der sogenannten 3. Welt sensibilisiert werden. Der soziale und gesellschaftliche Kontext stand dabei im Vordergrund. Bei letzterer stellte man das Einzelobjekt in seiner - mehr oder weniger - künstlerischen Ausführung in den Mittelpunkt und setzte es im Extremfall ganz für sich alleine in eine Vitrine. Der Anspruch an das Künstlerische und der ästhetische Genuß durch den Betrachter erschienen dabei besonders wichtig, die Information über das Objekt selbst sowie seine Funktion innerhalb der Kultur trat dabei in den Hintergrund. Ein gutes 
Beispiel dafür ist der Ausstellungskatalog des Rautenstrauch-Joest-Museums, der die hundert schönsten Objekte zeigen will und sich "Kunst der Welt" (Hrsg. v. Völger 1999) nennt. Darin ist auch eine zugegeben sehr schöne und interessante Speerschleuder aus Westaustralien abgebildet. Doch ist eine Speerschleuder, mag sie noch so schön sein, Kunst? Der Leser mag diese Frage für sich selbst beantworten.

Diese verschiedenen Methoden bezeichne ich hier als Ausstellungs"ideologien", da sie bestimmte Zeitgeistströmungen repräsentieren, die sich mitunter auch gegenseitig bekämpf(t)en. In jüngerer Zeit ist wieder eine Rückkehr $\mathrm{zu}$ völkerkundlich, d. h. gesellschaftlich eingebundenen Ausstellungen zu verzeichnen. Anhand dieser beiden Ausstellungsrichtungen läßt sich jedoch auch wieder das Spannungsfeld "Mensch versus Objekt" aufzeigen: Betrachten wir nur das Objekt in seiner künstlerisch-ästhetischen Form oder sehen wir dahinter die Menschen, die es benutzten, die es ja auch erfanden und fertigten, um ihren wirtschaftlichen, ihren sozialen Alltag oder ihre Religion $\mathrm{zu}$ gestalten?

\subsection{Wissenschaftlich-universitäre Anbindung}

Bis in die fünfziger und sechziger Jahre hinein ist eine Personalunion von Universitätsprofessoren und Museumsdirektoren an deutschen Instituten bzw. Museen zu verzeichnen. Das hat sich durch erhöhte Studentenzahlen und die umfangreicheren Aufgaben von Völkerkundemuseen heutzutage geändert. Bis auf wenige Ausnahmen existieren heute getrennte Personalstrukturen zwischen den universitären Instituten und den Museen. Obwohl dies für die zu erledigenden komplexen Arbeiten hie und da durchaus sinnvoll ist, so ist doch eine zunehmende Abschottung zu verzeichnen. Oftmals wissen die Museen nicht, was an den Instituten geschieht oder aktuell diskutiert wird, und die Universitätsstudenten erfahren nichts darüber, wie die museale Arbeit funktioniert - von einigen Praktika abgesehen. Auch berufliche Karrieren laufen bis auf wenige Ausnahmen völlig getrennt nach Museum bzw. Universität ab. Dies ist an sich ein beklagenswerte Zustand, der durch eine neue gegenseitige Zuwendung dringend behoben werden müßte.

\subsection{Rekonstruierender versus positivistischer Ansatz}

Der letzte Punkt führt mich zurück zum ersten Punkt: Stets waren Wissenschaftler und Objektesammler an einer herauszuschälenden Ursprungskultur interessiert, sie wollten die "eigentliche" Kultur herauskristallisieren. Sie, die Vertreter der westlichen Welt, versuchten, die traditionelle, präeuropäische Gesellschaft zu erfassen. Mit anderen Worten: Sie waren nichts anderes als Rekonstrukteure. ${ }^{14}$ Weniger interessierte sie das Leben, wie es zeitgenössisch ablief, wie und ob die Kinder zur Schule gingen, welche Sprache sie sprachen, in welchen Häusern sie lebten. Das Leben, so, wie es gerade passierte, so, wie man es phänotypisch wahrnahm, interessierte nicht. Mit anderen Worten: die positivistische Wahrnehmung des Alltags war kein Forschungsgegenstand.

Mir ist bewußt, daß Forderungen nach einem positivistischen Ansatz leicht mißverstanden werden und harsche Kritik hervorrufen können, da in der Ethnologie gerade von jungen Wissenschaftlern und Wissenschaftlerinnen immer wieder Analysen statt Beschreibungen gefordert worden sind. Die "Kaiser-Wilhelm-Land-Durchquerungs-Ethnologie" sei Vergangenheit, nun müßten theoretische Gesellschaftsanalysen her, so die Forderung neuerer Zeit und vielfach auch in der Gegenwart. Das ist zwar im Kern richtig, allerdings darf die soziale Realität der heute Lebenden dabei nicht ausgeklammert bleiben. Wie leben Aborigines - oder Torres Strait Islanders oder die Nachfahren der tasmanischen Aborigines - heute? Dies sind Fragen, denen man nicht aus dem Wege gehen kann. Sollten sie angeblich nicht zu den Inhalten der Ethnologie gehören? Sollten Soziologen oder gar Sozialarbeiter sich um diese Dinge kümmern? Ich meine nein. Die Ethnologie muß sich hier dringend ihr ureigenes Feld zurückerobern!

\footnotetext{
${ }^{14}$ Programmatisch kann hier der Satz von Justin Stagl gelten, der 1981 wie 1993 (S. 2/15ff) schrieb: "...die Ethnologie [hat] in ihrer klassischen Epoche (ca. 1920-1960) ihre wichtigste, weil nicht mehr wiederholbare Leistung vollbracht: die Bestandsaufnahme der Gesamtheit der Primitivkulturen vor deren Assimilation durch die 'Weltzivilisation'."
} 


\section{Zusammenfassung}

Der vorliegende Artikel spannt einen weiten Bogen von den Grundlagen der Ethnologie, der Entwicklung dieser Wissenschaft und ihren forschenden und sammelnden Aspekten bis hin zur heutigen Praxis der musealen Sammlungsbearbeitungen. In einem tabellarischen Raster wurde dabei versucht, einen Überblick herzustellen und auf die heutige Bedeutung und die Konsequenzen für Forschung und Ausstellungswesen hinzuweisen. Besonderes Augenmerk lag dabei auf den Australiensammlungen in deutschen Völkerkundemuseen - speziell dem Rautenstrauch-JoestMuseum in Köln - sowie der wissenschaftlichen Rezeption der ethnologischen Situation in Australien. Zum Abschluß wurden sieben Thesen vorgestellt, die einerseits Analysen des Museumsund Wissenschaftsbetriebes, andererseits Forderungen für die künftige Arbeit darstellen.

Wo wird die Zukunft der Völkerkundemuseen hinführen angesichts immer knapperer Mittel, angesichts einer Kulturpolitik, bei der es nie um Inhalte zu gehen scheint, sondern offenbar immer nur um Geld? Man darf gespannt sein!

\section{Literatur}

Erckenbrecht, Corinna, 1992. Feldforschung in einer australischen 'outback-town' oder "Takin' notes an' shit". kea. Zeitschrift für Kulturwissenschaften 3. 44-55.

Erckenbrecht, Corinna, 1993a. Wissenschaftsgeschichtlicher und religionsethnologischer Streit: Hochgottglaube versus autochthone Religionsformen am Beispiel der Kamilaroi, Australien. Anthropos, Internationale Zeitschrift für Völker- und Sprachenkunde 88. 517-528.

Erckenbrecht, Corinna, 1993b. Regionale Schwerpunktstudie: Die Kamilaroi im nördlichen New South Wales, in: Corinna Erckenbrecht, 1993. Frauen in Australien. "Aboriginal women" gestern und heute. Bonn: Holos-Verlag. 215-361.

Erckenbrecht, Corinna, 2000. Die materielle Kultur der australischen Aborigines - eine "Stein"zeit?, Newsletter der deutschen Gesellschaft für Australienstudien 14. 27-42.

Fenner, Burkhard, 1990. Nur für Eingeweihte - Zur Ausstellung geheimer Sakralgegenstände aus Australien. Kölner Museums-Bulletin. Berichte und Forschungen aus den Museen der Stadt Köln. 1/1990. 29-40.

Forster, Georg, 1777. A voyage round the world. London. 1778-80. Reise um die Welt. Deutsche Erstausgabe. 1983. Reise um die Welt. Herausgegeben und mit einem Nachwort von Gerhard Steiner. Frankfurt a.M.: Insel Taschenbuch.

Foy, Wilhelm, 1906-1910. Führer durch das Rautenstrauch-Joest-Museum. Cöln.

Gräbner, Fritz, 1905. Kulturkreise und Kulturschichten in Ozeanien. Zeitschrift für Ethnologie 37. 28-53.

Gräbner, Fritz, 1906. Wanderung und Entwicklung sozialer Systeme in Australien. Globus 90. 181186, 207-210, 220-224, 237-241.

Gräbner, Fritz, 1909. Die melanesische Bogenkultur und ihre Verwandten. Anthropos, Internationale Zeitschrift für Völker- und Sprachenkunde 4. 726-780, 998-1032.

Gräbner, Fritz, 1911. Methode der Ethnologie. Heidelberg.

Gräbner, Fritz, 1924. Das Weltbild der Primitiven. München.

Hauser-Schäublin, Brigitta, Krüger, Gundolf (Hrsg.), 1998. James Cook. Gaben und Schätze aus der Südsee. Die Göttinger Sammlung Cook/Forster. Gifts and Treasures from the South Seas. The Cook/Forster Collection, Göttingen. München, London, New York: Prestel Verlag. Deutschenglische Ausgabe.

Morgan, Lewis Henry, 1877. Ancient society or researches in the lines of human progress from savagery through barbarism to civilisation. New York.

Pützstück, Lothar, 1995. "Symphonie in Moll". Julius Lips und die Kölner Völkerkunde. Pfaffenweiler: Centaurus-Verlagsgesellschaft. Kulturen im Wandel 4.

Schmidt, Wilhelm, 1926. Die Sprachfamilien und Sprachkreise der Erde. Münster. 
Schmidt, Wilhelm, 1937. Handbuch der Methode der kulturhistorischen Ethnologie. Münster.

Schmidt, Wilhelm, 1912-1955. Der Ursprung der Gottesidee. 12 Bände. Münster.

Schmidt, Wilhelm, Koppers, Wilhelm, 1924. Völker und Kulturen. Regensburg.

Stagl, Justin, 1981. Szientistische, hermeneutische und phänomenologische Grundlagen der Ethnologie, in: W. Schmied-Kowarzik, J. Stagl, Hsg., Grundfragen der Ethnologie. Beiträge zur gegenwärtigen Theoriediskussion. Berlin: Dietrich Reimer Verlag. 1-38.

Stagl, Justin, 1993. Zweite, überarbeitete und erweiterte Auflage. Dort: S. 15-49.

Völger, Gisela, 1986. Die Wissenschaft lebt nicht von der Luft" - Bemerkungen zur Australiensammlung des Rautenstrauch-Joest-Museums. Wallraf-Richartz-Jahrbuch 47. 247-260.

Völger, Gisela, Hsg., 1999. Kunst der Welt im Rautenstrauch-Joest-Museum für Völkerkunde, Köln. München, London, New York: Prestel Verlag. 


\section{Introduction to George Seddon \\ Trevor Hogan, La Trobe University, Melbourne}

George Seddon (b. 1927) is Professorial Associate, Centre for Studies in Australian Literature, Department of English, University of Western Australia. In an era of "specialists without spirit", and of enterprise universities creating territory to ensure their fitness to survive, Seddon is that rare and endangered species that Thomas Carlyle once dubbed as "Professor of Things-in-General". He is one of the best known and revered Australian scholars of environmental studies having penned pioneering studies in regional geography, environmental history, and literary criticism, aesthetics and landscape.

Seddon was initially trained in English language and literature at The University of Melbourne (1950) In 1956 upon returning from several years freelance travelling and teaching at universities in Europe, Canada and the United States, what he calls a "customary bigfella walkabout", Seddon was appointed to a lectureship in English Literature at The University of Western Australia. He discovered, much to his initial disgust, that the local bush was nothing like what he had grown to love in his home state of Victoria in South-east Australia. Never one to disown his emotions or putting them aside without reflection, Seddon enrolled in undergraduate biological and earth sciences while carrying a full academic research, teaching and administrative load in the English Department. Such was his new found interest in the Swan Coastal Plain that he wrote two books on the subject and completed his Masters of Science and doctoral thesis in geology at the University of Minnesota (1964-66). Across five decades he has held Chairs in four different disciplines (English, UWA; Geology, Oregon; History and Philosophy of Science, UNSW; Environmental Science, Melbourne) and taught at universities in Lisbon, Toronto, Bologna, Rome, Venice, Minnesota, and Oregon in addition to Melbourne, Sydney and Perth in Australia. He initiated programs in the philosophy of science at The University of Western Australia (1966-1970) and taught history and philosophy of science at The University of New South Wales, Sydney (1971-1974). He initiated new programs in environmental studies and Landscape Architecture at The University of Melbourne where he was first appointed founding Director of The Centre for Environmental Studies (19741982), later becoming Dean of the Faculty of Architecture and Planning (1982-1987). He also officially launched the journal Landscape Australia (1977 - ).

Not only has Seddon crossed the disputed turfs and boundaries of academic professions, disciplines and departments but he has made a career out of working beyond the academy in community, regional and government consultancies and studies. At The University of Melbourne between 1974 and 1987, Seddon undertook studies on environmental assessment, landscape perception, urban design and conservation planning. He was particularly good at working at the complex intersections of nature-society struggles and debates. He took an avid interest in the contested terrains between metropolitan suburbs and arable agricultural hinterlands, or wilderness areas in key built-up population areas. He studied the development and routing of major power stations and transmission lines through ecologically and socially sensitive areas, the energy and infrastructure needs of national parks, and bicycle plans for cities. In 1979, with Ross King and Jeremy Pike he also wrote the first suburban history of its kind in Australia on Hawthorn, a significant Victorian era middle class and middle ring suburb in Melbourne. His enthusiasm for local history and cultural heritage found expression in his loving restoration of houses in which he has lived in Melbourne and Fremantle. He has combined this with a landscape ecologist's imaginative flair for garden design that incorporates native and exotic plants suited to local climate and soil conditions. One should not overlook his many practical handbooks and guides to vegetable gardens, historical and cultural heritage walks and house restoration.

Seddon as both regionalist and cosmopolitan is living testimony to the inadequacy of such dichotomous notions that to be regionalist is to suffer from volk yokel strains of communitarianism 
while to be a cosmopolitan is to necessarily seek the empty non-places of airport lounges and international hotels. Seddon's international reach in his various intellectual labours is as much extensive as it is a reflection of his sharp sense of place. For a good example - in addition to the collected essays below - see his essay on his "Return to Portugal' collected in Best Australian Essays 1998 (Melbourne, Bookman Press). He has undertaken regional and urban planning consultancies in such locales as Texas, China and Italy. In his study of Venice he demonstrates a typically acute awareness of the unintended consequences of human interventions on the environment, at one and the same time a love of unpicking common sense or paradigmatic views of cause and effects. In this case, Seddon questions the popular hypothesis that Venice is sinking by uncovering more specific human made causes of subsidence, namely, the unintended impacts of post-war dredging of ship canals coupled with the infilling of the landward margins of the lagoon.

Seddon is a superb stylist whose best metier is perhaps the essay, the review and the lecture podium where he can combine and share with others his acerbic wit, erudition, and critical enthusiasm in equal measure. As befitting his pragmatic, historical and comparative proclivities, his love of languages and landscapes, and his multi-disciplinary and multi-generic corpus of writings, Seddon rarely stops to spell out the conceptual underpinnings of his thinking. Nevertheless the lineaments of a social theory of landscape and society can be discerned in the way in which he builds historical and textual sociologies of perception. His thought is important for his central and consistent emphasis on holding two critical insights as true. First, that all human experience of nature is already inscribed by human language and past experiences contained in our language. Second, such has been the extent, range and long history of human impact on nature we must increasingly value nature for its own sake over against the stakes and interests of humans. Against new world ecocentric environmentalists, Seddon details the myriad ways in which even the most wild and remote margins of planet earth have been perceived, imagined and shaped by humans. Against the anthropocentric proclivities of economistic politicians and political economists, Seddon has explored the infinite and diverse otherness of natural ecosystems in which humans must perforce find their habitus.

The following essay is an exemplary thought-piece on one such remote region of northwest Australia. Seddon re-narrates the process and self-understandings of perceiving the Pilbara by European explorers, missionaries, miners and pastoralists through to our own contemporary journeys across the terrain in aesthetic, scientific and technological reappropriations of place and space. As these most recent explorations and imaginings unfold, Seddon gently leads the reader to appreciate the enduring presence of Aboriginal inscriptions and practices over at least forty thousand years even as he concludes that we still haven't done yet with Terra Australis Incognita. And we might add that we hope too that Seddon is not done yet with helping us rethink nature and our languages of nature on and at its antipodean margins, and elsewhere, everywhere.

\section{Bibliography}

A City and its Setting: images of Perth, Western Australia. (with David Ravine), 1986. Fremantle: Fremantle Arts Press.

Landprints: Reflections on Place and Landscape, 1997. Melbourne: Cambridge University Press.

Searching for the Snowy: An Environmental History, 1994. Sydney: Allen and Unwin.

Sense of Place: a response to an environment, the Swan Coastal Plain, 1972. Western Australia. Nedlands, W.A.: University of Western Australia Press.

Swan River Landscapes, 1970. Nedlands, W.A.: University of Western Australia Press.

Swan Song: Reflections on Perth and Western Australia, 1956-1995, 1995. Nedlands, W.A.: University of Western Australia Press.

[This article was first published in Thesis Eleven 69 (May 2001), 65-68, and is reprinted here with the kind permission of Sage Publications.] 


\section{Aktuelles aus Forschung und Lehre, Wirtschaft, Medien etc.}

\section{Das Australien-Exil deutschsprachiger Musiker: Kurzfassung eines Referats zum Symposium "Movements and Harmonies. Musical Bridges between Australia and Germany", Australienzentrum, 23. Februar 2001} Albrecht Dümling, FAZ und TU Berlin

Für Musiker war Australien nach 1933 kein bevorzugtes Exilland. Angesichts der geringen Zahl vorhandener Musikerstellen und der restriktiven Haltung der dortigen Musicians Union erhielten Instrumentalisten, Dirigenten, Komponisten und Sänger aus Deutschland oder Österreich in der Regel weitaus seltener die Einreise als Angehörige anderer praktischer Berufe. Die vom Hilfsverein der Juden in Deutschland herausgegebene Zeitschrift "Jüdische Auswanderung" warnte deshalb 1937 generell Künstler vor der Übersiedlung nach Australien. Neuere Studien australischer Historiker ${ }^{1}$ bestätigen die restriktive Haltung der damaligen Administration. Sogar die nach der Konferenz von Evian zugesagte Liberalisierung für deutsch-jüdische Flüchtlinge habe - so Malcolm Turnbull - angesichts der damit verbundenen Sonderbedingungen in Wahrheit eine Einschränkung bedeutet. $^{2}$ Die versprochene Quote von 15.000 Flüchtlingen wurde nie erfüllt, zumal mit Kriegsbeginn die Aufnahme weiterer Refugees endete. Ausnahmen bildeten lediglich die Internierten aus Großbritannien und Singapur. Angesichts dieser Einschränkungen überrascht, daß Australien überhaupt zu einem Zufluchtsland für Musiker wurde. Einen ersten Einblick in dieses noch wenig erforschte Thema gab die Konferenz "Musiker-Exil in Australien", die im Mai 1996 im Dresdner Zentrum für zeitgenössische Musik stattfand und deren Referate inzwischen im Druck vorliegen. $^{3}$

Im Rahmen eines von der DFG geförderten Forschungsprojekts am Zentrum der Antisemitismusforschung der Technischen Universität Berlin werden diese Untersuchungen gegenwärtig weitergeführt. Nach der Diskussion der Begriffe Exil, Emigration und Deportation bei einer jüngst von der TU Berlin durchgeführten Konferenz widmet sich dieses Referat den verschiedenen Wegen, auf denen vor dem NS-Staat geflohene Musiker in Australien landeten. ${ }^{4}$

Die angenehmste Form der Einreise bildete zweifellos die Konzertreise. Auf einer solchen trafen die Mitglieder des Spivakowsky-Kurtz-Trios, der Pianist Jascha Spivakowsky, sein Bruder Tossy, bis 1933 Konzertmeister des Berliner Philharmonischen Orchesters, und der Cellist Edmund Kurtz, am 4. Februar 1933 in Australien ein. Nachdem sie von dem wenige Tage zuvor erfolgten Machtwechsel in Deutschland erfahren hatten, entschlossen sie sich spontan zum Bleiben. Die Voraussetzungen waren gut, da Jascha Spivakowsky den fernen Kontinent bereits auf zwei erfolgreichen Konzertreisen kennengelernt und eine wohlhabende Australierin geheiratet hatte. Obwohl damit der Lebensunterhalt gesichert war, konnte die bisherige Karriere in Melbourne nur noch reduziert fortgesetzt werden, weshalb sich der Geiger und der Cellist schon wenige Jahre später zur Übersiedlung in die USA entschlossen. Für Jascha Spivakowsky, der in Melbourne blieb, bedeutete diese Entscheidung den Schritt in die künstlerische Isolation.

In seltenen Fällen hatten fluchtwillige Musiker ein Stellenangebot aus Australien erhalten. Rabbi Hermann Sänger, der selbst 1936 aus Berlin geflohen war, rettete den Berliner Dirigenten Hermann

\footnotetext{
${ }^{1}$ Vgl. Paul Bartrop: Australia and the Holocaust 1933-1945. Melbourne 1994; Hilary Rubinstein, 1988. The Jews in Australia. A thematic history. Vol. 1: 1788-1945. Port Melbourne.

${ }^{2}$ Malcolm Turnbull, 1999. Safe Haven. Records of the Jewish experience in Australia. National Archives Research Guide, S. 20.

${ }^{3}$ Vgl. A. Dümling, Hsg., 2000. Zu den Antipoden vertrieben. Das Australien-Exil deutschsprachiger Musiker. Saarbrücken. (Verdrängte Musik. Bd. 17)

4 A. Dümling: Zwischen Deportation, Exil und Emigration. Deutschsprachige Musiker in Australien. Vortrag zur Konferenz "Verfolgung, Rettung und Neuanfang. Jüdische Musiker und Komponisten im nationalsozialistischen Deutschland und in der Emigration", Berlin 2.12.2000.
} 
Schildberger, indem er ihm eine Stelle als Musikdirektor am Temple Beth-Israel, der liberalen Synagoge von Melbourne, anbot. Da die kleine Gemeinde aber nur ein winziges Gehalt zahlen konnte, mußte Schildberger den übrigen Lebensunterhalt mit einer großen Palette anderer musikalischer Aktivitäten verdienen, die von Privatmusikunterricht bis zu Chor-, Orchester- und Opernleitung reichten.

Musiker, die weder auf einer Konzertreise oder mit einer sonstigen Einladung einreisen konnten, mußten bei anderen Berufen Zuflucht suchen. Richard Goldner, bis 1938 Solobratschist in Hermann Scherchens Wiener Musica Viva-Orchester, hatte sich vergeblich um eine Flucht nach Nord- oder Südamerika bemüht. Das Australien-Permit erhielt er, weil sein Bruder ihn bei der Schmuckherstellung einsetzen konnte. Während des Krieges diente der handwerklich und technisch begabte Richard Goldner der australischen Armee mit technischen Erfindungen, bevor er 1945 endlich zu seinem Musikerberuf zurückkehren konnte. Auch Alfons Silbermann, der nach seinem Jurastudium eine Kapellmeisterausbildung begonnen hatte, erhielt nur mit der falschen Berufsangabe "Koch" die Einreise. Nach dem Kriege wurde er kurzfristig Dozent am Konservatorium von Sydney, bevor er wieder nach Europa zurückkehrte.

Mehrere der aus Deutschland oder Österreich geflohenen Musiker bewarben sich vergeblich um eine Stelle in Australien, wobei auch fremdenfeindliche und antisemitische Motive eine Rolle spielten. Das eklatanteste Beispiel ist der Fall von Arnold Schönberg, einem der bedeutendsten Komponisten des 20. Jahrhunderts und bis 1933 Leiter einer Meisterklasse an der Preußischen Akademie der Künste. Da ihm in den USA nur schlechtbezahlte Stellen angeboten worden waren, bewarb er sich 1934 um eine Position als Lehrer für Harmonielehre und Musiktheorie am Konservatorium von Sydney. Aus zwei handschriftlichen Eintragungen auf Schönbergs Bewerbungsformular geht hervor, daß er nicht etwa wegen mangelnder Qualifikation abgelehnt wurde. Die Eintragungen lauten "Jewish" und "Modernist ideas and dangerous tendencies". Als jüdischer und zugleich avantgardistischer Komponist war Schönberg für das damalige Australien offenbar nicht akzeptabel. In einer glücklicheren Lage befanden sich die siebzehn Kinder, die im Juli 1939 mit einem Kindertransport in Australien eintrafen. Eines dieser Kinder, der aus Wuppertal stammende George Dreyfus, wurde später, nachdem er zunächst als Orchestermusiker seinen Lebensunterhalt gesichert hatte, der erste freischaffende Komponist Australiens.

Nachdem Großbritannien 1940 alle "feindlichen Ausländer" inklusive der aus Deutschland und Österreich geflohenen Juden interniert hatte, versprach Australien, 6000 dieser Internierten aufzunehmen. Aber nur ein einziges Schiff, die "Dunera", unternahm schließlich die weite Reise. Trotz des unglücklichen Verlaufs dieser Deportation, der erneut Internierungen folgten, entschlossen sich schließlich 913 Männer zum Verbleib in Australien. Zu ihnen gehörten mehrere Musiker, darunter der jüdische Kantor Boas Bischofswerder und sein Sohn Felix. Ebenfalls im September 1940 trafen auf der "Queen Mary" ca. 200 Internierte aus Singapur in Melbourne ein. Einer von ihnen war der Pianist und Organist Werner Baer, der mit Hilfe seiner jungen Frau im Dezember 1938 aus dem KZ Sachsenhausen nach Singapur hatte fliehen können. Da er dort inzwischen eine sichere Position als Dozent und Organist erhalten hatte, entsprach die Deportation nach Australien keineswegs seinen Vorstellungen. Ebenso enttäuscht war er, daß er- wie die anderen Internierten - in einem Lager untergebracht und zum berufsfremden Arbeitseinsatz verpflichtet wurde.

Daß Künstler und Intellektuelle, die vor Hitler ins Ausland geflohen waren, in Australien zunächst für mehrere Jahre hinter Stacheldraht leben mußten, stieß bei den Betroffenen auf scharfen Widerspruch. Der Protest konnte sich auf unterhaltende Weise äußern, wie etwa in den witzigen Songs von Ray Martin oder der Revue "Reise um die Welt", zu der Hans Werner Katz die Musik komponierte. Er konnte aber auch aggressivere künstlerische Formen annehmen, wie etwa in der dissonanten 1. Symphonie, die Felix Werder, der Sohn des jüdischen Kantors Boas Bischofswerder, 1943 im Lager Tatura schuf. Interessanter Weise blieb Werder in Australien, während der anpassungsbereite Ray Martin bei der nächsten Gelegenheit nach England zurückkehrte. Als Komponist und Musikkritiker übernahm Werder eine wichtige Rolle im australischen Musikleben. 
Das von Richard Goldner 1945 in Sydney gegründete Musica Viva Kammerorchester wurde zur Keimzelle der Musica Viva Australia, der größten Kammermusikorganisation der Welt. Werner Baer, für den Australien keineswegs ein Traumziel bedeutet hatte, bereicherte mit seiner vielseitigen Musikbesessenheit bis zum letzten Atemzug das Kulturleben dieses Kontinents. Auch Musikbegeisterte wie Charles Berg, Stefan Haag, Joseph Krips oder Hans Zander leisteten trotz aller Schwierigkeiten, mit der sie einst nach Australien gekommen waren, schließlich wesentliche Beiträge zu dessen Musikkultur. 


\section{Hörsaal und Studio: Eindrücke eines sechsmonatigen Arbeitsaufenthaltes "Downunder"}

\section{Irene Quaile-Kersken, Deutsche Welle, Köln}

Nach regelmäßigen Urlaubsreisen sowie einigen dienstlichen Aufenthalten für die Deutsche Welle z.B. für die Vorbereitung und Durchführung des 1. Deutsch-Australischen Mediensymposiums, 1998 - beschloss ich im letzten Jahr, ein halbes Jahr "Downunder" zu verbringen. Ein Programm von Vorträgen sowie einige Reportageaufträge von verschiedenen Rundfunkanstalten, vor allem aber die zeitweilige Übernahme der Europasendung von ABC Radio National, machten den Aufenthalt auch für meinen Arbeitgeber, die Deutsche Welle, interessant, so dass sie bereit war, mir für die Dauer des Aufenthaltes unbezahlten Urlaub zu gewähren.

Auf Wunsch des Herausgebers werde ich hier einige Erfahrungen aus diesen sechs Monaten zusammenfassen, in denen ich die Chance hatte, die australischen Medien 'von Innen' kennen zu lernen. Dieser Beitrag ist als subjektiver Erfahrungsbericht zu lesen und stellt keine Ansprüche an Vollständigkeit oder Wissenschaftlichkeit.

\section{Vorträge an Universitäten:}

Meine erste Station war die QUT (Queensland University of Technology) in Brisbane. Sie hat eine journalistische Abteilung, die sich nicht nur von der akademischen Seite sehen lassen kann. Praktische Erfahrung wird hier groß geschrieben, so dass man sich sehr über die Gelegenheit freute, eine europäische Journalistin als 'Gastdozentin' zu begrüßen. U.a. gehören der ehemalige Europakorrespondent der ABC, Lee Duffield, sowie der ehemalige ABC-Intendant, Brian Johns, zum Lehrpersonal von QUT.

Als ich das Büro, das mir für die Dauer meines Aufenthaltes in Brisbane zur Verfügung gestellt wurde, betreten wollte, machte ich kurz Halt vor dem Schwarzen Brett. Hatte ich da nicht ein mir gut bekanntes Logo entdeckt? Gesucht wurden Studenten, die bereit sind, neben ihrem Studium für den Universitätsradiosender zu arbeiten. Hervorgehoben wurde die Sendung 'Undercurrents', das Flagschiff der 'Community' Sender, zu dem auch QUT regelmäßig beiträgt. Geworben wurde mit dem Erfolg zweier junger Journalistinnen, die durch ihre Mitarbeit an dieser Sendung das journalistische Handwerk so gründlich erlernt hatten, dass sie sich erfolgreich um ein sechsmonatiges Journalistenpraktikum - bei der Deutschen Welle in Köln - beworben hatten!

Diese Möglichkeit für junge JournalistInnen hatte ich vor meiner Abreise ins Leben gerufen. Die erste Journalistin war schon in meiner Redaktion. Die zweite lernte ich später in Melbourne kennen. (Die erste hat inzwischen einen Zweijahresvertrag im aktuellen Dienst der DW, die zweite wird für die nächsten Monate unsere Jugendsendung moderieren, eine dritte berichtete kürzlich vom Weltwirtschaftsgipfel in Salzburg.)

Bei QUT wurde ich u.a. von der 'Media Policy Unit' zu einem Vortrag über die Entwicklung des Auslandsrundfunks in Deutschland eingeladen. Das Publikum (Dozenten, Doktoranden, Journalisten aus der Umgebung) interessierte sich sehr für die Entwicklungen seit dem Fall der Mauer sowie die Zukunftsperspektiven der Deutschen Welle. Die Fragen aus dem Publikum im Anschluss an den Vortrag thematisierten aber auch das Schicksal des australischen Auslandssenders Radio Australia, dessen Budget und Programm vor einigen Jahren drastisch gekürzt wurden. Vor diesem Hintergrund war das Publikum sehr an den Überlebenschancen und -taktiken der Deutschen Welle interessiert. Auch die technischen Entwicklungen und Möglichkeiten - Satellit, Digitalisierung und Internet-Radio - erweckten großes Interesse.

Beeindruckt hat mich bei meiner Arbeit in Brisbane die technische Ausstattung dieser Hochschule. In den Vorlesungssälen hat man sämtliche technische Mittel zur Verfügung. Die Möglichkeiten, Nachrichten aus dem Internet als Text oder Audio, Videoeinlagen, oder Unterlagen direkt aus dem PC einzusetzen sowie eine computerisierte Beleuchtungsanlage machen jeden Vortrag - bei entsprechender Vorbereitung, versteht sich - zu einem Multimediaereignis, der dafür sorgt, dass die Studenten keine Chance haben, einzuschlafen. 
Die Journalismusstudenten in Brisbane sind von hohem Kaliber. Bei den Vorlesungen und Seminaren, die die Auswahl, Vorbereitung und Verfassung von Nachrichten zum Thema hatten, zeigten sie überwiegend ein reges Interesse am Weltgeschehen und an journalistischen Prinzipien. Dies kann man allerdings nicht allgemein von den angehenden australischen Journalisten behaupten. Andernorts konnte ich nur staunen, dass der Name Slobodan Milosevic einigen völlig unbekannt war, aber auch Personen des öffentlichen Interesses im etwas näher liegenden asiatischen Region, so z.B. die Nobelfriedenspreisträgerin Aung San Su Chi in Myanmar/Burma. Auch die geographischen Kenntnisse anderer Kontinente lassen oft zu wünschen übrig ("Wo liegt eigentlich Kalkutta?"). Im großen und ganzen stößt man auch immer wieder auf ein stark inländisch geprägtes Interesse in den Medien. Der Mangel an europäischen Themen bei den meisten heimischen Radiosendern erklärt teilweise den Erfolg des Englischen Dienstes der Deutschen Welle in ihrer Zusammenarbeit mit dem Nachrichtensender ABC NewsRadio. So wird das DW Nachrichtenmagazin mehrmals täglich von diesem Sender ausgestrahlt.

\section{Bei der ABC}

In Melbourne übernahm ich zeitweise die Redaktion und Moderation der wöchentlichen Europasendung von ABC Radio-National (RN). Diese Sendung ist wohl einmalig in Australien. Für 40 Minuten widmet sich 'The Europeans' ausschließlich dem entfernten Kontinent. Interviews am Telefon stellen einen Hauptteil des Materials für die Sendung da - etwas anders als in meiner Heimatredaktion, wo die Entfernungen zu den Interviewpartnern nicht ganz so groß sind und man auch Korrespondenten vor Ort einsetzt. Durch den Zeitunterschied ist der Tagesablauf ebenfalls anders, da Europa erst in den australischen Abendstunden wach wird. So ist eine Spätschicht die Regel. Die Hörer interessieren sich für eine breite Themenpalette. Kernenergie, der Zypernkonflikt, die Zeitungskultur in Europa, das neue schottische Parlament, aber auch die Skandale um die deutschen Christdemokraten fanden alle das Interesse der Hörer. Neben der Produktion der Hörfunksendungen liefern die RN-Journalisten über das Internet ein beachtliches begleitendes Service-Angebot an Informationen sowie Antworten an Höreranfragen.

Während meines Aufenthaltes fand gerade eine große Umstrukturierung des öffentlich-rechtlichen Senders ABC unter dem neuen Intendanten Jonathan Shier statt. Als Außenstehende und freiberufliche Mitarbeiterin hatte ich die Möglichkeit, die Entwicklungen zu beobachten, ohne direkt betroffen zu sein. Die vom neuen Management durchgeführten Personaländerungen sind sehr kontrovers und wurden ständig in den Medien thematisiert. Die Ergebnisse bleiben abzuwarten.

\section{Vorbereitung des 2. Deutsch- (Europäisch-) Australischen Mediensymposiums}

In Melbourne und in Sydney konnte ich weitere Vorbereitungen für ein zweites Mediensymposium treffen. 1998 hatte ich für die Deutsche Welle in Zusammenarbeit mit dem Goethe Institut Sydney und ABC NewsRadio das erste Deutsch-Australische Mediensymposium organisiert und durchgeführt. (Die GASt-Mitglieder Professor Stilz, Bader und Leitner nahmen teil). Die nächste Veranstaltung sollte eine europäisch-deutsche Veranstaltung unter Mitwirkung der EABC (Zusammenschluss der europäischen Handelskammern in Australien) sein. Aus finanziellen Gründen kann die Veranstaltung in diesem Jahr nicht mehr stattfinden. Die Finanzierung wird für das kommende Jahr wieder beantragt.

\section{Sonstiges}

Zwischen diesen Aktivitäten hatte ich auch die Gelegenheit, Vor-Ort-Recherchen für Hörfunk- und Zeitungsbeiträge durchzuführen. $\mathrm{Zu}$ den nennenswertesten gehören wohl eine Reise zum Great Barrier Reef (Themen: Ökologie, Tourismus) sowie zu den Opalminen in Lightning Ridge.

Besonders aufgefallen ist mir die Gastfreundschaft der australischen KollegInnen sowohl im akademischen als auch im Medienbereich. Selten brauchten wir ein Hotelzimmer. Uns wurden wiederholt Häuser zur Verfügung gestellt. Als Bezahlung musste man meistens in Abwesenheit der Hausbesitzer lediglich Garten und Katzen versorgen. Ganz so einfach ist es nicht, wenn ich in Deutschland eine Unterkunft für die GastjournalistInnen suche! 
Ich würde mich freuen, von GASt-Mitgliedern zu hören, die sich für die in diesem kurzen Bericht angeschnittenen Themen interessieren. Ich möchte vor allem den auf Wunsch des australischen Botschafters in Berlin (damals noch in Bonn) sowie des deutschen Botschafters in Canberra ins Leben gerufene Journalistenaustausch gerne ausweiten, aber auch unseren jungen JournalistInnen die Möglichkeit geben, mit StudentInnen über ihre Arbeit und Erfahrungen in Australien und Deutschland zu diskutieren. Selbstverständlich würde ich mich über Interesse an Kooperationen bei der Planung und Durchführung des nächsten Mediensymposiums freuen. 


\section{New York Festivals: Two gold medals for DW-Radio: Feature series of the programme Man and Environment receives the United Nations Award}

\section{Dr. I. Quaile-Kersken, Deutsche Welle, Englisches Programm}

For the first time Deutsche Welle (DW), Germany's international broadcaster, has won the 'United Nations Award' in gold for environment programming at the 'New York Radio Festivals'. The same feature series of the English service of DW- radio won a second Gold medal at the same festival. This was announced by DW on 22 June 2001. More than 1,200 entries from 36 countries were submitted for the international competition. The United Nations Award is presented jointly by the UN Department of Public Information and the New York Festivals for outstanding achievement in radio programming which best exemplifies the ideals and goals of the United Nations. This year the jury chose the DW-Feature "Man and Environment: WWF eco- regions" as the winning entry.

Feature editors Irene Quaile-Kersken and John Hay of the DW-radio/English service are responsible for the twelve-part series that is at present broadcast monthly. It was partly produced in conjunction with the Australian broadcaster ABC Radio National. The editor responsible there is Maria Zijlstra.

The idea and concept of the series on endangered species and habitats were based on the 'Global 200 programme' of the World Wide Fund for Nature (WWF). It introduces listeners to key eco-regions on all continents, providing an insight into the links between ecology, development and the economy. At the same time, listeners learn about the significance and the biological diversity of the approximately 200 key eco-regions selected by the WWF. 


\section{Bericht über das Forschungsprojekt Environmental concerns in Australian poetry: An exploration of Australia's psychocultural history Norbert H. Platz, Universität Trier}

Von Oktober 2000 bis März 2001 erlaubte mir ein Stipendium der Volkswagenstiftung, mich an der University of Western Australia (Perth) in ein Projekt mit dem Arbeitstitel Environmental concerns in Australian poetry: An exploration of Australia's psychocultural history einzuarbeiten.

\section{Beschreibung des Vorhabens}

Gemäß der bei der Antragstellung vorgelegten Projektbeschreibung sollte zunächst einmal ein bibliographischer Überblick über australische Gedichte vorgelegt werden, in denen Einstellungen zur Natur und zu ökologischen Fragestellungen implizit oder explizit thematisiert werden. Dies erwies sich als ein arbeitsaufwendiges Unterfangen. Um einen repräsentativen Überblick zu gewinnen, mußten an der Reid Library der University of Western Australia in Perth zunächst einmal eine Vielzahl von Gedichtanthologien und Veröffentlichungen individueller Autoren erfaßt werden, bevor eine konkrete Auswertung und Erfassung von Gedichttexten erfolgen konnte.

\section{Forschungsbedingungen}

Als besonders günstig erwies sich das Bestehen von Datenbanken, bei denen man über elektronische Suchverfahren zu Ergebnissen gelangte. Hilfreich waren vor allem die onlineKataloge der Reid Library sowie der Westaustralischen Staatsbibliothek ("The Library and Information Service of Western Australia") sowie die Möglichkeit zur elektronischen Kontaktaufnahme mit Setis (http://setis.library.usyd.edu.au/) an der Mitchell Library in Sydney.

\section{Vorläufige Ergebnisse}

Von etwa 800 erschlossenen Titeln wurden 100 Anthologieveröffentlichungen für die nähere Analyse ausgewählt. Bis zum Ende der in Australien durchgeführten Recherchen konnten ca. 80 Anthologien gründlich ausgewertet werden. Etwa 750 Gedichttexte sind bereits bibliographisch erfaßt und mit auswertungsrelevanten Kurzbeschreibungen versehen worden. Jede dieser Kurzbeschreibungen basiert auf gründlicher Lektüre des jeweiligen Gedichttextes.

Die geplante Bibliographie wird unter dem Titel "The Green Dimension in Australian Poetry: A Bibliographical Guide for Study and Research" in der zweiten Jahreshälfte als Website Publication international zugänglich gemacht werden. Das Format für die Bibliographie ist bereits ausgearbeitet und lehnt sich an das Modell an, das für die bereits bestehende Website "The Green Dimension in Canadian Poetry: A Bibliographical Guide for Study and Research" gewählt wurde. (vgl. http://www.uni-trier.de/uni/fb2/anglistik/Projekte/platz/greendimension/index.html)

\section{Vorläufige Erkenntnisse}

In der bisherigen Forschung wurden die australischen Einstellungen zur Natur und Landschaft häufig dem thematischen Oppositionspaar Hölle oder Paradies zugeordnet. Gerade weil im 19. Jahrhundert die Landschaft oft als "Hölle" identifiziert wurde, konnten sich bei der Landnutzung viele Einstellungen entwickeln, die eine ökologiefeindliche Praxis begünstigten - z.B. weitflächige Brandrodungen, Vernichtung der Regenwälder und Verringerung des Baumbestandes, "bindungslose Landausbeutung" (d.h. die Farmer blieben auf dem ihnen zugewiesenen Land bis zu dem Zeitpunkt, wo es unfruchtbar wurde, und pachteten anschließend noch unerschlossene Landflächen). Die Paradies-Vorstellung bevorzugte eine die realen australischen Lebensbedingungen beschönigende Sehweise. Zugegebenermaßen spiegeln viele der bibliographisch erfaßten Gedichte diese antagonistischen Perspektiven wider. Andererseits belegt die Mehrzahl der erfaßten Gedichte jedoch auch einen völlig anderen Erfahrungsmodus von natürlicher Umwelt, der auf konkreter empirischer Beobachtung basiert. Natürliche Umwelt wird hier nicht in das Einordnungsschema von Hölle oder Paradies eingezwängt, sondern als ein Bereich empirischer Beobachtung und konkret erfahrener psychischer Qualitäten wahrgenommen. Die Autorinnen und Autoren dieser Gedichte erweisen der natürlichen Umwelt eine neugierige Wertschätzung. Implizit oder explizit enthalten die Texte relevante Werturteile über die in der 
Umwelt erfahrenen Phänomene. Oft reflektieren die Gedichte auch darüber, wie das Individuum in der natürlichen Umwelt seine eigene Identität gewinnt und einen Heimatbezug erfährt. Bekanntlich ist "home" eine zentrale Kategorie im Diskurs der postkolonialen Kulturen.

Im Unterschied zu dem im öffentlichen Diskurs in den Vordergrund gerückten quantifizierbaren Nutzen des Landes betonen die Gedichte ein subjektiv erfahrbares eudämonistisches Potential. Die Gedichte kontrastieren den Anspruch auf Gewinn mit dem Anspruch auf "happiness", d.h. eine Befindlichkeit von "Glück", die sich aus einem Bemühen um ein Leben im Einklang mit der natürlichen Umwelt ergibt. Da gerade in der neueren Umweltethik die Frage nach der menschlichen Eudämonie wieder thematisiert wird, würden sich im australischen Kontext viele dieser Gedichte dafür eigenen, das Verhältnis von menschlichem Glücksbedürfnis und Motivation zu umweltgerechtem Verhalten neu zu thematisieren.

\section{Zusammenarbeit mit australischen Forschergruppen}

Mein Projekt stellte ich auf einem Symposium des International Centre for Landscape and Language (http://www.ecu.edu.au/ses/research/scapes/index.html) an der Edith Cowan University (Perth) vor. Mit Vertretern der Forschungsgruppe Landscape and Language stand ich seit November 2000 in Kontakt. Eine Intensivierung unserer Kooperation im Rahmen eines internationalen Forschungsprojekts ist geplant. Ebenso ist eine Zusammenarbeit mit dem von Prof. Helen Tiffin und Dr. Ruth Blair initiierten Forschungsvorhaben "Environmentalism and Literary Studies" an der School of English, Media Studies and Art History (University of Queensland, Brisbane) vorgesehen. 


\title{
Justice in water re-allocation in Australia: A psychological approach Dr. Geoffrey J. Syme and Blair E. Nancarrow
}

The following abstract is of a colloquium presented to the Department of Psychology at the University of Trier on 21/11/2000 by Dr. Geoffrey Syme and Blair Nancarrow of CSIRO's Australian Research Centre for Water in Society (ARCWIS), Perth, WA. As director of ARCWIS Dr. Geoffrey J. Syme and his team are specialized in institutional, organizational and community analysis of water related policy and its implementation and evaluation. Blair E. Nancarrow as the operational manager of the Australian Research Centre for Water in Society is responsible for the design and management of large scale and national projects. Their work includes research in the field of perceived justice in public involvement programs especially of water allocation and reallocation processes in Australia. The talk summarized a variety of studies relating to fairness in natural resource allocation policy. These have been conducted over the last decade. The CSIRO researchers also used the trip to Trier to discuss and develop ongoing collaborative research with PD Dr. Elisabeth Kals and Prof. Dr. Leo Montada.

\begin{abstract}
:
"Australia is facing an increasing problem of competition for access to water. Early settlers had assumed that there would be plenty for everyone. However, in recent years, environmental degradation has been caused by the overuse of many of Australia's major water resources for agricultural irrigation and other industrial and development purposes. This has caused conflict between different States in Australia, and among a wide variety of interest groups. The Federal Government has responded by mounting a nationwide water reform program to provide for environmental (in-stream) flows, efficient use of water through economic market mechanisms, and where necessary, re-allocation to bring surface water and groundwater use back to sustainable levels. In some cases, this re-allocation could result in substantial hardship for individual irrigators and their rural communities.

While there is much political rhetoric declaring that this adjustment will be undertaken equitably, there is considerable confusion as to what this means. The available water law has, until now, been based on British common law, and has had difficulty in coping with the "public interest". There is also substantial confusion on how justice can be incorporated into the rules of the economic market, and even whether it should be a consideration. Finally, the role of social impact assessment in interpreting and evaluating the outcome of water reform is confused.

In this presentation it was described how the elements of a fairness heuristic were derived from community research to evaluate the justice considerations associated with a major groundwater reallocation. The issues associated with incorporating this empirical research into government reallocation policy are discussed in association with the role of psychologists in this area."
\end{abstract}




\section{Der Australienforscher aus der Mark Brandenburg. Seit über 10 Jahren wird das Andenken an Ludwig Leichhardt wachgehalten Bernd Marx, Boblitz}

Seit über zehn Jahren wird das Andenken an Ludwig Lechhardt wachgehalten. Niemand ahnte wohl bei der Gründung der Gesellschaft Ludwig Leichhardt e.V: Trebatsch am 2. Oktober 1990, dass sich die Ortschaft an den Ufern der Spree zu einem wahren Zentrum der Pflege und Wahrung des Vermächtnisses des Australienforschers Friedrich Wilhelm Ludwig Leichhardt im Lande Brandenburg entwickeln würde. "Es ist uns gelungen, das Andenken an den in unserer Gemeinde am 23. Oktober 1813 geborenen Naturwissenschaftler bei der Bevölkerung wachzuhalten und weitere Quellen für ein noch breiteres Lebens- und Schaffensbild zu erschließen", so Herr Andre Parade, Vorsitzender der Gesellschaft bei den Feierlichkeiten, anlässlich des 10. Jahrestages der Vereinsgründung. Auf Anregung motivierter Leichhardtfreunde und zahlreiche Heimatforscher aus der Gemeinde Trebatsch und der Region wurden neue interessante Initiativen im Verlaufe der letzten zehn Jahre aus der Taufe gehoben. Besonders hinsichtlich der Vorbereitungen zum 150. Todestag des Australienreidenden im Jahre 1998 wurde in einer umfangreichen Bandbreite die Öffentlichkeit über das Leben, Wirken und geheimnisvolle Ende des Entdeckers und Forschers berichtet. Seit Januar 1997 wurden über 300 Veranstaltungen, Vorträge, Ausstellungen, Tage der offenen Tür sowie Exkursionen in Deutschland und in Australien durchgeführt. $\mathrm{Zu}$ den erfolgreichsten Sonderausstellungen und Dokumentationen zählte:

- "Australien - Sehnsucht und Schicksal Ludwig Leichhardts",

- "Friedrich August Schmalfuß - Künstler, Schwager, Freund",

- "Ludwig Leichhardt - im Spiegel der Presse",

- "Entdecker und Entdeckungen der Welt" und

- "Die Welt ist nur ein Kleeblatt" in der Gemeinde Trebatsch sowie in den Städten Potsdam, Cottbus, Lübben und Senftenberg.

Insgesamt konnten über 20.000 Interessierte sich bei den Ausstellungen und Vorträgen mit dem Lebensweg und Schicksal des preußischen Wissenschaftlers vertraut machen, darunter über 2.000 Kinder und Jugendliche. Auch Publikationen, Aufsätze und Artikel sind über Ludwig Leichhardt und die Vereinsarbeit in der Oder-Spree-Region erschienen. Mittlerweise gibt es einen engen Kontakt und umfangreichen Informationsaustausch $\mathrm{zu}$ zahlreichen Institutionen in Australien. Schwerpunkt bildet gegenwärtig die langfristige Vorbereitung des 190. Geburtstags des bedeutenden Australienforschers im Jahre 2003. Die Gesellschaft Ludwig Leichhardt e. V. Trebatsch hat es sich zur Aufgabe gemacht, in den nächsten Monaten das vorhandene Leichhardt Archiv zu erschließen und die Ergebnisse in einer Ausstellung der Öffentlichkeit zu präsentieren. Die Mitglieder werden auch in Zukunft alles unternehmen, um die Erinnerungen an den bedeutenden Sohn der Gemeinde bei der Bevölkerung wachzuhalten und bei der jungen Generation das Interesse zu wecken. 


\section{Peitzker, Tania, 2000. Dymphna Cusack (1902-1981): eine feministische Analyse von Geschlecht/Gender in ihren romantischen realistischen Texten. ${ }^{.}$}

Das Dissertationsprojekt befaßt sich mit der australischen Autorin Dymphna Cusack, deren Popularität in Ost und West zwischen 1955 und 1975 ihren Höhepunkt erreichte. In diesem Zeitraum wurde sie nicht nur in den westlichen Industriestaaten, in Australien, England, Frankreich und Nord Amerika viel gelesen, sondern auch in China, Rußland, der Deutschen Demokratischen Republik und in vielen Sowjetrepubliken. Im Verlauf ihres Schaffens wurde ihr große Anerkennung für ihren Beitrag zur australischen Literatur zuteil; sie erhielt die "Commonwealth Literary Pension", die "Queen's Silver Jubilee Medal" und 1981 den "Award of her Majesty". Trotz dieser Unterstützung durch den Staat in Australien und England äußerte Cusack immer wieder feministische, humanistisch-pazifistische, und anti-faschistisch bzw. pro-sowjetische Sozialkritik.

Sie war auch für ihren starken Nationalismus bekannt, plädierte dafür, eine "einheimische" Literatur und Kultur zu pflegen. Besonders das australische Bildungssystem war das Ziel ihrer Kritik, basierend auf ihren Erfahrungen als Lehrerin in städtischen und ländlichen Schulen, die sie ihrer Autobiographie beschrieb. ${ }^{2}$ Weder ihr Intellekt, noch ihre Seele oder ihre Körper wurden gefördert, um ganze Männer oder ganze Frauen aus ihnen $\mathrm{zu}$ machen. Besonders letztere wurden vernachlässigt. Mädchen wurden ermutigt, ihren Platz dort zu sehen, wo deutsche Mädchen ihn einst zu sehen hatten: bei Kindern, Küche, Kirche. ${ }^{3}$

Cusack engagierte sich stark für Bildungsreformen, die das Versagen australischer Schulen, das erwünschte liberal-humanistische Subjekt herauszubilden, beheben sollten.

Der liberale Humanismus der Nachkriegszeit schuf ein populäres Bedürfnis nach romantischem Realismus, den man in Cusacks Texten finden kann. Um verstehen zu können, wie Frauen sich zwischen "Realismus und Romanze" verfingen, biete ich eine Dekonstruktion von Geschlecht innerhalb dieses "hybriden" Genres an. Mittels feministischer Methodik können Einblicke in die konfliktvolle Subjektivität beider Geschlechter in verschiedenen historischen Perioden gewonnen werden: die Zeit zwischen den Kriegen, während des Pazifischen Krieges und den Weltkriegen, während des Kalten Krieges, zur Zeit der Aborigine-Bewegung, des Vietnamkrieges, sowie zu Beginn der zweiten feministischen Bewegung in den siebziger Jahren.

\footnotetext{
${ }^{1}$ Die Dissertation ist ungekürzt auf der Webseite veröffentlicht: http://pub.ub.uni-potsdam.de/2000meta/0012/door.htm.

${ }^{2}$ Dymphna Cusack war Gymnasiallehrerin für Englisch bis 1944, als einer schwere Krankheit sie zur Invaliden machte und sie ihre Manuskripte bis an ihr Lebensende diktieren mußte.

${ }^{3}$ Dymphna Cusack, 1991. A window in the dark, Hsg. Debra Adelaide. Canberra: National Library of Australia) 104.
} 


\section{Dresden-Besuch des australischen Komponisten George Dreyfus, April 2001 Rosemarie Gläser, Dresden}

Der australische Komponist George Dreyfus, der das Musikleben des Fünften Kontinents durch Film- und Fernsehmusiken, Chorwerke, kammermusikalische Werke, Symphonien und Vertonungen von Gedichten und Balladen maßgeblich geprägt hat und in Deutschland durch die Uraufführungen seiner Opern Rathenau (Kassel 1993) und Die Marx Sisters (Bielefeld 1996) bekannt geworden ist, besuchte im April 2001 Deutschland mit mehreren eigenen musikalischen Veranstaltungen. Vom 20.-30. April war er in Dresden. Schon 1996 hatte G. Dreyfus die Elbestadt besucht, als er - zusammen mit dem ebenfalls aus Melbourne kommenden Komponisten Felix Werder - an dem Kolloquium "Musiker-Exil in Australien" (Leitung: Dr. Albrecht Dümling) im Dresdner Zentrum für zeitgenössische Musik teilnahm. Die beiden Komponisten wurden durch ein Künstlerporträt und eigene Kompositionen vorgestellt (Felix Werder Klavier; George Dreyfus Fagott). Sie hielten Vorträge über ihre Emigrationserfahrungen.

George Dreyfus, 1928 in Wuppertal als Kind wohlhabender deutsch-jüdischer Eltern geboren, konnte-zusammen mit seinem älteren Bruder und fünfzehn weiteren jüdischen Kindern-im Sommer 1939 nur durch einen Schiffstransport nach Australien der faschistischen Verfolgung entkommen. Auch den Eltern gelang die Flucht, während die Großeltern in Auschwitz umkamen.

Die Veranstaltung im Dresdner Zentrum für zeitgenössische Musik am 21. April 2001 war abermals ein "Künstlerporträt" und eine Wiederbegegnung mit Dresdner Musikwissenschaftlern. Im Mittelpunkt des Programms standen der Dokumentarfilm Life is Too Serious, den der australische Regisseur und Produzent Don Parham über den Komponisten in dreijähriger Zusammenarbeit gestaltet hat, und das Solostück Odyssey for a Lone Bassoon, interpretiert von George Dreyfus selbst. Prof. Dr. Rosemarie Gläser gab eine Einführung in Leben und Werk des Komponisten und Zusammenfassung des Dokumentarfilms, der bereits am 7. Januar 2001 von der ABC ausgestrahlt und von ca. 250000 Zuschauern gesehen wurde. Der Film vermittelt aus der Sicht der Musikkritiker Phillip Adams und Clive O'Connel, des Komponisten Peter Sculthorpe (Sydney), des Schwarzaustraliers Tom Lewis, der in dem Sextet for Didjeridu and Wind Instruments (1971) als Didgeridoo-Spieler aufgetreten ist, sowie weiterer Personen aus dem Musikleben Australiens Einblicke in das Leben und Wirken des Komponisten. Sein Solovortrag auf dem Fagott war ein lebendiges Beispiel seines Schaffens.

Eine einprägsame Begegnung hatte Dreyfus am 23.4.01 mit Anglistik-Studenten der TU im Rahmen der Vorlesung "Socio-Cultural Studies of Australia" (Prof. Dr. Gläser), wo er über "Australian Aboriginal Music" sprach. Den Auftakt bildete eine Darbietung des Germanistik-Studenten Stephan Schreiber auf seinem Didgeridoo. Es folgte ein Tonbandbeispiel aus der Sammlung Uluru von Tony O'Connor, wo das Didgeridoo von Naturgeräuschen des Monsunregens begleitet wird. Im Anschluß daran sprach G. Dreyfus über die Kompositionsprinzipien seines Sextet for Didjeridu and Wind Instruments. Erstmalig wird in diesem kammermusikalischen Werk das Instrument der Ureinwohner ein gleichberechtigtes Partnerinstrument im Ensemble (west)europäischer Blasinstrumente und damit in ein Gesamtwerk integriert. Die Studierenden gewannen interessante Einblicke in den kompositorischen Aufbau des Sextetts, was das Verständnis des sich anschließenden Tonbandvortrags wesentlich erleichterte.

Den Höhepunkt der Veranstaltungen mit G. Dreyfus bildete das Benefizkonzert zum Aufbau der neuen Dresdner Synagoge, das am 27. April 2001 im Kulturrathaus Dresden-Neustadt in Gegenwart des stellvertretenden australischen Botschafters, John Richardson, stattfand. Das Programm "Open House" beinhaltete elf Titel, die hauptsächlich aus Film- und Fernsehmusiken stammen, darunter die populären Stücke Rush ("Goldgräberstimmung") und die Kinderfernsehserie Sebastian the Fox, aber auch das erinnerungsträchtige Larino - Safe Haven, das dem Kinderheim Larino am Stadtrand von Melbourne, wo die jüdischen Kinder im Sommer 1939 ihre erste Zuflucht fanden, gewidmet ist und das 1989, bei einem Wiedersehen der einstigen Emigranten nach 50 Jahren, uraufgeführt 
wurde, und nicht zuletzt die Vertonung eines der Galgenlieder von Christian Morgenstern (Das Knie). Die Mitwirkenden waren neben dem Komponisten, der das Fagott spielte, zwei Studenten der Musikhochschule Dresden: Raphael Gärtig (Flöte) und Lucas Kunze (Bariton), außerdem die Konzert-Gitarristin Maria Gerloff, Geschäftsführerin des "Freien Musikvereins Paukenschlag" in Dresden-Klotzsche. Der Schauspieler Olaf Burmeister vom Staatsschauspiel Dresden hatte die Rolle des Erzählers zwischen den einzelnen Stücken übernommen. Prof. Gläser gab zu Beginn eine Einführung in das Leben und Werk des Komponisten.

Die Musikveranstaltungen fanden eine gute Aufnahme und anerkennende Pressekritiken. Sie förderten die Begegnung zwischen unterschiedlichen Musikkulturen und erweiterten den Gesichtskreis der Teilnehmer. Dem Komponisten war es ein persönliches Anliegen, Aufführungen in der Semperoper und in der Komödie und ein Benefizkonzert in der Unterkirche der Dresdner Frauenkirche zu besuchen. Er lernte das Richard-Wagner-Haus in Graupa und das Weberhaus in Hosterwitz kennen. A historischen Stätten machte er sich vertraut mit dem Werk der im Dritten Reich verfemten Schriftsteller Erich Kästner und Viktor Klemperer. Vor seiner Weiterreise besuchte er Terezin in Nordböhmen, wo sich das Ghetto und das Konzentrationslager Theresienstadt befanden. Dort wurden seine Großeltern interniert, ehe sie nach Auschwitz deportiert wurden. Die Gespräche zwischen G. Dreyfus und dem Förderverein zum Aufbau der Dresdner Synagoge und die Zusammenarbeit mit Dresdner Künstlern waren von dem gemeinsamen Willen getragen, ein Beispiel zu geben im Sinne der Losung "Brücken bauen - Versöhnung leben", die an der Baustelle der wiedererstehenden Dresdner Frauenkirche zu lesen ist. 


\section{Australische Kunst in Norddeutschland}

\section{Elisabeth Bähr, Speyer}

Zwei sehr unterschiedliche Ausstellungen (die eine in der Städtischen Galerie Wolfsburg, die andere im Sprengel Museum Hannover) beschäftigen sich mit der Gegenwartskunst von Künstler/innen australischer Aborigines. Die Ausstellung in Wolfsburg, zu der die Aboriginal Art Galerie Bähr aus Speyer die Exponante als Leihgaben stellt, zeigt Arbeiten von Künstler/innen aus Zentralaustralien und aus den Kimberleys; darunter Werke der international bekannten Johnny Warangkula Tjupurrula, Gloria Petyarre, Eubena Nampitjin, Elizabeth Nyumi und Mick Namarari Tjapaltjarri.

Der Titel der Ausstellung "Das Verborgene im Sichtbaren" leitet sich aus der Tatsache her, daß die Künstler/innen nicht das Sichtbare abbilden, sondern aufgrund eines überlieferten Wissens um die existentiellen und komplexen Beziehungen zwischen dem Menschen, seinem Land und der den Dingen innewohnenden Spiritualität Themen in individueller künstlerischer Weise interpretieren. Während die Städtische Galerie Wolfsburg Arbeiten mit Acryl auf Leinwand und Erdpigmeten auf Baumwolle zeigt, präsentiert das Sprengel Museum Hannover Bilder auf Rinde sowie Skulpturen aus Ramingining (Arnhem Land).

\section{Entstehung der Gegenwartskunst}

1971 malte Mick Namarari Tjapaltjarri das erste Bild mit Acryl auf Leinwand. Dies war (von einigen Vorläufern abgesehen (der Beginn einer Kunstbewegung, die sich innerhalb weniger Jahre sprunghaft verbreitete. Nach 15 Jahren bereits, also Mitte der 80er Jahre hatten alle großen Kunstmuseen Australiens Sammlungen mit dieser Malerei angelegt und zeigen seitdem Exponate in den Abteilungen für zeitgenössische Kunst ihrer ständigen Ausstellungen. Die Auseinandersetzung darum, ob es sich um zeitgenössische Kunst oder um sog. Volkskunst handelt, ob man sie in die Kategorien Art Brut oder Ethnographica preßt (was in Deutschland so gern getan wird), war damit weitgehend zugunsten der Zeitgenossenschaft entschieden.

Die Western Desert in Australien sieht auf den ersten Blick nicht so aus, als sei sie eine Gegend, die Anfang der 70er Jahre eine der aufregendsten Kunstbewegungen der Moderne hervorbrachte. Ein Zusammentreffen verschiedenster gesellschaftlicher Vorgänge begünstigte die Kunstbewegung, die ihren Ausgangspunkt in Papunya nahm, einer Siedlung ca. $250 \mathrm{~km}$ westlich von Alice Springs gelegen. Diese Siedlung wurde als eine der letzten 1961 unter dem Diktat der Assimilationspolitik der australischen Regierung gegründet. Die Aborigines sollten sich der weißen Lebensweise anpassen (so sie denn die schrecklichen Verfolgungen bis hin zum Völkermord bis weit in das gerade vergangene Jahrundert hinein überlebt hatten). Ungeachtet ihrer unterschiedlichen Kulturen wurden bis Mitte der 60er Jahre etwa 1000 Aborigines verschiedener Völker in Papunya zusammengepfercht, was notwendigerweise zu sozialen Spannungen führte.

Das Bewußtsein bei den Älteren der Aborigines darüber, daß ihre Kultur zunehmend Schaden leidet, ließen einen Ausweg dringend erscheinen, und die Mitte der 60er Jahre beginnende Landrechtsbewegung hatte weiteren Einfluß auf das wachsende Verlangen der Aborigines nicht nur auf Wahrung ihrer Kultur, sondern auf die Einforderung ihrer Anerkennung. Sobald der Zugang zu Malmaterialien, zu Acrylfarben und Leinwand geschaffen war, verbreitete sich die Kunstbewegung sprunghaft von Siedlung zu Siedlung entlang der sehr weitläufigen Verwandtschaftsbeziehungen und entlang der Reisen zu religiösen Feierlichkeiten. Seit Beginn der Kunstbewegung arbeiten die Künstler/innen für die Öffentlichkeit. Die enstandenen Werke wurden also nie für einen anderen Zweck als der Vermittlung von Kunst und Kultur gearbeitet. Sie reflektieren nicht vergangene Traditionen, sondern die heutige Kultur. Es sind keine religiösen Objekte. Die Arbeiten sind Teil der zeitgenössischen Kunst Australiens; sie sind eine der zwei Säulen, auf denen die australische Gegenwartskunst basiert.

\section{Die gezeigten Kunstrichtungen}

Die Ausstellung in der Städtischen Galerie Wolfsburg zeigt drei Richtungen der Desert-Kunst: Farbflächenmalerei mit Erdpigmenten auf Baumwolle von Künstler/innen aus Warmun; die 
farbenfrohen Acrylbilder aus Wirrimanu (Balgo) und Arbeiten der klassischen Punkt- und Linienmalerei bzw. deren Weiterentwicklungen.

Abgesehen von der Verwendung gleicher Malmaterialien, nämlich der seit alters her verwendeten Erdpigmente, finden die Künstler/innen aus Warmun ihren jeweils eigenen unverwechselbaren Stil. Die Bearbeitung der Erdpigmente zu verschiedenen Farben und in unterschiedlicher Konsistenz münden in ganz eigene Malrichtungen. Die perspektivisch "richtige" Anordnung wird ignoriert, und es ist nicht ungewöhlich, in der gleichen Arbeit Vorder-, Seiten und Aufsichten zu vereinen.

Die Werke aus Wirrimanu (Balgo) zeichnen sich durch den Sinn der dortigen Künstler/innen für starke Farbkontraste und für die Betonung von Brillianz aus. Leuchtende Farben werden in harten Kontrasten voneinander abgesetzt. Die jeweils individuelle Ausformung zeigt sich z.B. in einem sehr pastosen Farbauftrag, um einen haptischen Eindruck hervorzurufen, oder in einer Technik des Verbergens und Hervorhebens durch den Auftrag übereinanderliegender Farbschichten.

Als Merkmal der Kunst Zentralaustraliens galt lange Zeit die Punktmalerei. In den klassischen Werken sind die Punkte als Hintergrund zu interpretieren, vor dem die Symbolik ihre Bedeutung entfaltet. Allerdings zeigen Künstler/innen heute auch völlig andere Richtungen in Bildern, die durch den Auftrag unterschiedlich großer Punkte in verschiedenen Farben und Dichten Strukturen zeigen, in denen die Punkte selbst Bedeutung gewonnen haben. Auch hier spielen Techniken des Verbergens und Hervorhebens eine Rolle. Eine weitere Richtung sind Linienbilder, die sich immer durch äußerst knappe Kompositionen auszeichnen.

\section{Tjukurrpa und Malerei}

Tjukurrpa, ein Wort, das in manchen Bildtiteln auftaucht, ist ein komplexes System von Vorstellungen über Weltbild und Religion, die allesamt in Wechselbeziehungen und Wechselwirkungen zueinander stehen. Tjukurrpa manifestiert sich zunächst in den Ahnen, die vor unendlicher Zeit das Land duchwanderten, die Landschaft formten, die Menschen und ihre Fähigkeiten, Tiere und Pflanzen schufen und schließlich in das Land zurücksanken, Teil des Landes wurden und noch heute gegenwärtig sind. Geographische Merkmale markieren Episoden von den Wanderungen der Ahnen. Oft sind diese Merkmale heilige Stätten, die es zu schützen gilt.

Die Reisen der Ahnen, die zusammen mit ihren Handlungen in Liedzyklen besungen und in Geschichten erzählt werden, verbinden weit entfernte Landteile miteinander und damit verschiedene Völker der Aborigines. In religiösen Zeremonien werden diese Handlungen und Wanderungen durch Gesänge, Tänze oder Bodeninstallationen belebt. Die Geschichten und Liedzyklen enthalten all das Wissen über Tiere, Pflanzen und Nahrung, über Medizin und über die Moral, die das Zusammenleben und das Überleben von Menschen ermöglicht. Die Geschichten und Liedzyklen sind nichts Mystisches, sondern dienten sowohl der Wissensvermittlung als auch der Geschichtsschreibung.

Tjukurrpa bedeutet aber weitaus mehr. In den Überzeugungen der Aborigines gibt es keine Spaltung zwischen Spiritualität und dem Materiellen, zwischen natürlich und übernatürlich. Tjukurrpa meint sowohl die Schöpfung als auch die sozialen Regeln des Zusammenlebens. Sie verbindet Vergangenheit, Gegenwart und Zukunft. Der Mensch ist Teil der Tjukurrpa wie die Tjukurrpa Teil des Menschen ist, seine geistige, seelische und materielle Identität. Tjukurrpa ist aber auch das Gesetz, das von den Ahnen gegeben wurde. Tjukurrpa ist nicht statisch. Das Neue der sich ändernden Umwelt, Vorfälle der Verfolgungen der Aborigines in der neueren Geschichte werden aufgenommen und zum Bestandteil der Tjukurrpa. Denn alles Neue ist in Wahrheit so alt wie die Welt selbst, weil es im Plan der Tjukurrpa bereits angelegt war.

Jede/r Aborigine besitzt eine oder mehrere Tjukurrpas im Sinne von Geschichten, die die spirituelle Verbindung zu einem bestimmten Teil des Landes darstellen; Land wird nicht besessen, sondern lediglich verwaltet und bewahrt. Jeder Mensch gehört zu einem bestimmten Teil des Landes wie umgekehrt das Land zu ihm gehört.

Tjukurrpa ist wahr; sie entspricht ebenso einer vorfindbaren Realität wie einer hinter den Dingen stehenden Spiritualität. Die Maltechniken des Verbergens und Hervorhebens finden in eben diesem 
Wechselspiel ihre Entsprechung. Land, Tjukurrpa, Mensch und Geschichte, also auch die im Gemälde erzählte Geschichte, sind miteinander verwoben. Bei einem solchen Hintergrund ist es kein Wunder, daß die Bilder unabhängig davon, in welcher Weise sie gemalt sind, immer eine große Präsenz und Kraft entfalten.

"Das Verborgene im Sichtbaren", Städtische Galerie Wolfsburg (Schloß), bis 14.10.2001, Offnungszeiten: Di 10-20, Mi-Fr 10-17, Sa, So 10-18, Programm unter www.aboriginal-art.de

"The Native Born", Sprengel Museum Hannover, bis 9.9.2001, Öffnungszeiten: Di 10-20, Mi-So $10-18$ 


\section{Rezensionen}

\section{Sally Morgan, 1987. My place. Fremantle, WA: Fremantle Arts Centre Press. Rez. von Ernst Reiner, Gummersbach}

1987 erschien dieses Buch in Fremantle (W.A.), sehr rasch wurden Nachdrucke notwendig, 1988 gab es 3 weitere Auflagen und 1999 war das Buch über 4oo.ooo mal verkauft worden. Im Jahre 2000 dürften es mehr als 500.00o Exemplare sein, die verkauft wurden. Auch in England und in den U.S.A. hatte dieses Buch zahlreiche Käufer gefunden. Wenn wir annehmen, daß zu den 50o.0oo Käufern je zwei weitere Leser kommen, also 1,5 Millionen Leser in einem Land mit 15 Millionen Einwohner, so hat jeder 1o. das Buch gelesen. Wie kommt es zu einer solchen Begeisterung für ein Buch, für eine ganz schlichte Familiengeschichte? Die Kritiken für dieses Buch sind zahllos und alle positiv. Im Vorspann zu dem zu besprechenden Exemplar werden 15 Beispiele an Kritiken zitiert.

Sally Morgan schildert schlicht und ohne stilistische Besonderheit ihr Leben und das ihrer Familie. Das Besondere daran ist die Entwicklung, die sie nimmt, denn als Mischling erlebt sie als Kind, daß sie von der Gesellschaft nicht den rein europäischen Kindern gleichgestellt ist. Die Mutter und Großmutter, die nach dem Tode des Vater, der an den Folgen des Krieges immer krank war, versuchen sie und ihre Geschwister zu erziehen, versuchen ihre Herkunft zu vertuschen, sie glauben zu machen, sie seien von indischer Abkunft.

Sally aber ist auf der Suche nach ihrer und ihrer Familie Identität. Deutlich weiß sie ihren Weg durch die Schule, ihre Jugend zu schildern und als Studentin sich zu ihrer Idendität als Aboriginee $\mathrm{zu}$ bekennen. Hier setzt nun die Suche nach der Vergangenheit der Familie ein gegen den Widerstand von Mutter und Großmutter.

Systematisch weiß sie von einem der Verwandten seine Geschichte auf Tonband aufzunehmen. Als die Mutter diese Geschichte hört, ist auch sie bereit ihre Vergangenheit zu erzählen. Sie reist mit der Familie - Sally ist inzwischen verheiratet und hat zwei Kinder, nach Norden von West-Australien in den Raum Marble Bay und zu der Station Corunna, wo ihr Stamm seine Heimat hat. Sie erfahren die vielen Verwandtschaftsverbindung, werden willkommen geheißen und bei jeder Begegnung erfahren sie Neues, was Sally nur ahnt. Die Aufzeichnungen Arthur's, einem Großonkel, ihrer Mutter und zum Schluß von der Großmutter kurz vor ihrem Tode zeigen die Tragödie der Eingeborenen, ihre Behandlung durch die Weißen. Die Schlichtheit, nicht ohne einen gewissen Humor, mit der die Berichte wiedergegeben werden, nehmen den Leser gefangen.

Sally Morgan hat hier eine, ihre Lebensgeschichte geschrieben, die sich jeder zueigen macht. Gerade dieses mag den Erfolg dieses Buches bewirkt haben.

Man versteht nun, - aber das ist das Problem, das diese Autobiographie aufgegriffen hat -, warum heute der Aboriginee Australiens nach den vielen Jahren des Verachtens durch den Weißen seiner selbstbewußt um die volle Anerkennung und Gleichstellung mit den Weißen kämpfen läßt. Dieses Buch von Sally Morgan hat sicher auch zum besseren Verständnis der Eingeborenen beigetragen. Seine Ehrlichkeit überzeugt! 
Davidson, Jim, Peter Spearritt, 2000. Holiday business. Tourism in Australia since 1870. Melbourne: Melbourne University Press, 416 S. - ISBN 0-522-84884-2. Rez. von Christof Pforr, University of Canberra, Belconnen, A.C.T.

Holiday Business erzählt die Geschichte des Tourismus in Australien und erhebt zugleich den Anspruch, das erste Buch dieser Art zu sein. Es ist jedoch mehr als nur eine Auflistung touristischer Ereignisse seit 1870, denn es erläutert zugleich sozio-kulturelle Aspekte touristischer Entwicklung, verweist auf ökologische Dimensionen und erklärt geographische Zusammenhänge.

Dieser reich illustrierte Band versucht durch detailierte Recherchen, beispielsweise anhand touristischer Attraktionen wie den 'Blue Mountains', der 'Gold Coast' oder des 'Roten Zentrums', Veränderungen, die der Tourismus in Australien in den letzten 130 Jahren erfahren hat, zu skizzieren. Hierbei werden verschiedene historische Stationen durchlaufen, von den fühen Anfängen der Kolonie, als Reisen noch Priveleg der reichen Oberschicht war, über die Strandkultur der 1950er Jahre, Ausdruck von Spass und Lebensfreude vieler Australier, bis hin zum heutigen Tag, an dem das Land eine wichtiges Ziel internationaler Touristenströme ist. Tourismus ist zum Massengeschäft und somit auch zu einem entscheidenden Eckpfeiler der australischen Wirtschaft geworden. Das Ausmass der Veränderungen wird deutlich, wenn man bedenkt, dass beispielsweise Ayers Rock heutzutage von mehr als 350000 Besucher jährlich aufgesucht wird, bis Ender der 1950er Jahre jedoch gerade einmal bis zu 100 Reisende angelockt hat.

Trotz seines akademischen Anspruchs ist es den Autoren, beide Professoren an Universitäten in Melbourne, gelungen, eine faszinierende Sozialgeschichte zu verfassen, die vor allem (aber nicht nur) all diejenigen fesseln wird, die sich für die Geschichte des Reisens und des Tourismus auf dem fünften Kontinent interessieren. 
Leitner, Gerhard, Bruce Bennett, eds. Australian Studies: A topic for tertiary education? [= Australia Centre Series 4]. Berlin: Berliner Debatte Wissenschaftsverlag, 1999. ISBN 3931703-47-9.

\section{Rezensiert von Cornelia Schulze, Universität Wuppertal}

Der vorliegende Band ist das Ergebnis einer im Oktober 1999 durchgeführten Konferenz, die in gemeinsamer Regie von der Gesellschaft für Australienstudien e.V. und dem Australienzentrum (ehemals Universität Potsdam) durchgeführt wurde. Die Veranstaltung widmete sich der grundsätzlichen Frage, ob und in welcher Weise Australienstudien (AS) an Universitäten implementiert werden sollten. Im Rahmen dieser Konferenz wurde die eigenständige Berechtigung von AS zunächst in Frage gestellt, doch dann überzeugend begründet.

Der Mut zur Bestandsaufnahme dessen, was schon existent ist, was in Zukunft Inhalt und Ziel sein müsse und wie sich dies mit der bestehenden Infrastruktur an heimischen Universitäten und Institutionen vertragen könnte, macht diesen Sammelband zu einem außergewöhnlichen Leseereignis. Nicht Selbstbeweihräucherung, sondern Selbstanalyse und -kritik stehen im Mittelpunkt der kontroversen Diskussion. Jeder einzelne Beitrag ist von Akademikern verfasst worden, die auf unterschiedliche Weise AS betreiben. Somit geht es nicht allein um eine theoretische Diskussion von tatsächlichen oder möglichen Inhalten dieses Studiengangs, sondern auch um Formen der Vermittlung. In diesem Band versammeln sich Beiträge aus verschiedenen Disziplinen: der Literaturwissenschaft, Linguistik, Didaktik, Geographie, Rechtswissenschaften und Geschichte. In seinem Vorwort mit dem gleichnamigen Titel des Bandes führt Gerhard Leitner in die Debatte ein. Zunächst bietet er einen kurzen Überblick zur Entwicklung von Regionalstudien als Thema der tertiären Bildung in Deutschland, die bis in die 1930er Jahre zurückgeht. Mit der Diskussion um die Institutionalisierung von AS widmet sich dieser Band einer spezifischen Region: Australien. Angesichts der finanziellen Engpässe an den Universitäten und der Tendenz, kürzere und verschultere BA/MA-Studiengänge einzuführen, die "Schlüsselqualifikationen" vermitteln sollen, anstatt Spezifizierung oder Vertiefung zu ermöglichen, muss die Frage beantwortet werden, welchen Nutzen die Implementierung von AS für die akademische Welt wie auch den Arbeitsmarkt hätte. Erstmals seit den 1968er Jahren steht die Universität wieder im Mittelpunkt des öffentlichen Interesses, und sie befindet sich in der ungemütlichen Position, sich rechtfertigen zu müssen, inwieweit sie die Bedürfnisse des Arbeitsmarktes bei der Ausbildung junger Akademiker berücksichtigt. Nicht Elitenbildung ist gefragt, sondern finanzierbare, arbeitsmarktgerechte und zügige Ausbildung der "Massen". Im Rahmen der zunehmenden Globalisierung und Ausweitung des Arbeitsmarktes auf das Ausland wird der Ruf nach internationalisierten, standardisierten und interdisziplinären Studiengängen laut. In diesem Zusammenhang müssen Befürworter von AS begründen, warum die Implementierung dieses neuen Studiengangs bereichernd sein würde. Die zentrale Frage ist demnach die Durchführbarkeit und Verwertbarkeit von AS an deutschen Hochschulen mit dem Blick auf bestehende Institute in Deutschland und Australien.

Die ersten fünf Beiträge dienen der Orientierung und zeigen, wie AS als ein Modul in einem erweiterten Bereich der Englischstudien integriert werden könnten. Bruce Bennett eröffnet die Diskussion mit seinem Aufsatz "Literary and Cultural Studies as a Curriculum Tool," in welchem er aus australischer Sicht aufzeigt, welche Themen und Inhalte für deutsche Studierende sinnvoll sein könnten. Die Auseinandersetzung mit der australischen Literatur und Kultur könne einen Zugang zur australischen Lebenswirklichkeit bieten und kontroverse Themen wie die Rolle Australiens im Asien-Pazifik-Raum oder den Umgang mit nationalistischen und rassistischen Tendenzen in einer multikulturellen Gesellschaft beleuchten. Gerhard Stilz untersucht in "Australia's Cultures: a Literary Approach, or Teaching Literary Topography" die Rolle der literarischen Topographie, die er als Subdisziplin der Literaturwissenschaft mit politischer Dimension begreift: "[L]iterary Topography should be considered more as the discipline which consciously and critically integrates and keeps alive the public discourse on a foreign country. Literary topography has therefore an eminently political function" (20). Susan Pfisterer gelingt es mit "An Antipodian Boldness: Australian Literature and International Curricula", die Relevanz australischer Inhalte für deutsche 
Universitäten aufzuzeigen. Für sie ist "reading, writing and studying Australian literature 'away from home' [is] a political process as well as an educational, cultural and social one" (29). Anschließend stellt sie in groben Zügen ein Kurrikulum zusammen. Gerhard Leitners "Australian Linguistics: a Module in Australien Studies" bietet erstmals eine systematische Untersuchung der gesamten Sprachsituation Australiens sowie der Nachbardisziplinen der Linguistik. Er bietet eine Definition der AS als "a scientifically-grounded study of the continent and nation within ist own network of relations" (37). Rosemarie Gläser reflektiert über ihre eigenen Erfahrungen mit einer interdisziplinären Vorlesungsreihe zu sozialen und kulturellen Aspekten Australiens und berichtet, dass das studentische Interesse für australische Themen und Inhalte in Zukunft noch ausbaufähig sei.

Die Vermittlung von AS steht im Mittelpunkt der beiden folgenden Aufsätze. Volker Raddatz verweist in "Curriculum Design and Teaching Strategies: Australia as an Interdisciplinary Theme for Tertiary and Secondary Education" darauf, dass die Inhalte von AS mit aktuellen Entwicklungen in der Fremdsprachendidaktik korrespondieren. Somit könnten AS an bestehende Studieninhalte angebunden werden. Da ein interkultureller Vergleich zwischen Australien und Deutschland notwendiges Handwerkszeug sei, böten AS einen hohen didaktischen und erzieherischen Wert. Sigrid Luchtenberg zeigt in "On the Role of Australian Multicultural Education in German Universitites" auf, wie Multikulturalismus in das australische Bildungssystem transportiert wurde und inwiefern dieses Modell sich auch auf deutsche Universitäten anwenden ließe. Ferner stellt sie eine Auswahl von Themenbereichen vor, die in die Ausbildung integriert werden müssten.

Als Geograph widmet sich Reinhold Grotz in "Geography - An Approach to Australian Economy and Society" der Rolle der Geographie innerhalb der AS. Überzeugend präsentiert er diese als eine Wissenschaft, die von Natur aus multidisziplinär sei und eine Vielzahl verschiedener Berufsbilder bediene. Er geht ausführlich auf die Bedürfnisse des Arbeitsmarktes ein und folgert, dass die Fähigkeit, in Netzwerken denken zu können den Marktwert der Graduierten gegenüber solchen erhöhen würde, die traditionelle Fächer studierten.

Margret Carstens diskutiert in "Australian Studies in Law: Indigenous Rights on the National, Comparative and International Level; Its Relevance for Legal Studies in Germany" die häufigen Mißachtungen der Rechte der Ureinwohner Australiens im Kontext des internationalen Rechts und untersucht die Lehr- und Lernsituation in Deutschland anhand dieses konkreten Beispiels. Sie folgert daraus: "If one wants to play the global game, one has to learn new rules, and include human, minority and indigenous rights" (117). Kevin Williams ergänzt ihre Diskussion der Rechtslage der australischen Urbewohner mit seinem Beitrag "Native Title in the Australian Context. Historial Denial and Legal Recognition." In diesem stellt er zunächst die sich wandelnde Rolle der Landrechte dar, um dann auf die potentiellen Auswirkungen der in Australien gefällten Rechtsentscheidungen auf das deutsche Rechtssystem hinzuweisen. Seine Darstellung der Entwicklung des Landrechts anhand von konkreten Gerichtsurteilen verdeutlicht, wie AS inhaltlich und methodisch aufbereitet und an Hochschulen vermittelt werden können.

Manfred Brusten berichtet in "Australia in Research and Teaching in the Social Sciences" über den aktuellen Status der AS in den Sozialwissenschaften. Seine Analyse auf der Basis eigener empirischer Untersuchungen führt zu dem Schluss, dass in diesem Fachgebiet keine systematische Verbindung zu Australien existiert, sich jedoch eine Vielzahl von Themen anbieten würden, um fehlende Brücken zu schlagen.

So wie die Konferenz in eine Plenumdiskussion mündete, bietet Bruce Bennett mit seinem "Round Table: Implementing Australian Studies in Germany" eine leserfreundliche Zusammenfassung aller Argumente und Ergebnisse der konstruktiven Diskussion um die Zukunft von AS. Auf der Basis der zusammengetragenen Argumente und Vorschläge zweifelt Bennett nicht daran, dass AS an deutschen Universitäten für Lehrende und Studierende aus verschiedensten Disziplinen attraktiv wäre. 
Als Sahnehäubchen des Bandes bieten Peter Spearritt und David Dunstan in "Sydney: Capital of the Continent" eine Hommage an Australiens "heimliche Hauptstadt". Sie verweisen auf die Sonderrolle Sydneys als australische Metropole und betonen, dass insgesamt $90 \%$ der australischen Bevölkerung in Küstenstädten leben. Die Kenntnis über die Hintergründe für diese ausgeprägte Form der Städteentwicklung sei unverzichtbar für das grundsätzliche Verständnis Australiens. Die Wahl der - offiziellen oder heimlichen - Hauptstadt hinge von komplexen politischen, ökologischen und infrastrukturellen Bedingungen ab. Als Vergleich für die eigene Situation ziehen sie Deutschlands Entscheidung hinzu, Berlin wieder zur Hauptstadt Deutschlands zu erklären. In beiden Fällen, so die Autoren, müssten verschiedenste Faktoren einbezogen werden, die zu dieser Entwicklung geführt hätten. Anhand des internationalen Vergleichs würden universale Einblicke in zugrundeliegende komplexe Zusammenhänge ermöglicht, von denen beide Seiten profitieren könnten.

Aufgrund der qualitativ hochwertigen, informativen und stimulierenden Beiträge übertrifft dieser Band die Erwartungen an eine interdisziplinäre wissenschaftliche Debatte im begrenzten Rahmen einer Monographie. Zudem liefert er zugleich eine theoretisch fundierte wie auch praktisch orientierte Basis für die Institutionalisierung von AS an deutschen Hochschulen. So beantwortet Australian Studies: A Topic for Tertiary Education? die eingangs gestellt Frage mit einem klaren "Ja!" und zeigt Wege auf, die möglichst bald beschritten werden sollten. 


\section{Schneider, Edgar, ed., 1997. Englishes around the world. Studies in honour of Manfred Görlach, 2 vols. Amsterdam: Benjamins. Vol. 1, pp vi+320; vol. 2, pp vi+357. Rezensiert von Gerhard Leitner, Freie Universität Berlin}

A festschrift is a collection of papers that reflect the interests of the contributors and the diversity of the research of the scholar who is celebrated by and in that book. A festschrift for a scholar of the stature of Manfred Görlach is bound to be more diverse than many, given his interest in English worldwide and historical linguistics. The editor has grouped the contributions around general, comparative themes (vol. I) and large geographic regions. Vol. I deals with the English of the British Isles and North America. Vol. II covers the Caribbean, Africa, Asia, Australia and New Zealand. There are gaps, of course. There are no papers on the South Pacific, none on Europe, except the one by C. Stephan on German students' abilities in identifying English varieties-he reports that NZE and AusE ranked low on the identification scale-and none on the internal diversity of the Antipodes. For these gaps, however sad as they are, the editor cannot be blamed. Given the context of this review, I will focus on those studies that are on or touch upon Australia. K. Hansen and A. Gupta discuss terminological issues that arise when one tries to characterize nations, speakers, functions of language and the linguistic texture of English worldwide. A. Gupta surveys the transplantation, acceptance and functions of English and suggests five types of English-using countries, viz. (i) monolingual ancestral English countries, (ii) monolingual contact variety countries, (iii) multilingual scholastic English countries, (iv) multilingual contact variety countries, and (v) multilingual ancestral English countries. They are exemplified (in this order) by Australia, Jamaica, India, Papua New Guinea and South Africa. It may be admissible to cast a wide net, to simplify the complex linguistic situation of a country, to overlook internal heterogeneity, yet it is unclear what application her grid may have. Two papers might have included Australia. J.-M. Gachelin looks at how the progressive and habitual aspects are expressed in non-standard Englishes in many varieties of English. He mentions non-standard Caribbean pidgins and creoles but ignores AusE and NZE. J. Holm et al. deal with passive-like constructions and ignores Kriol or Torres Strait creole (and the older pidgins).

Vol. II contains L. Bauer's papers on the Scottish influence on NZE and G. Tulloch's on AusE. Bauer's study, though on NZE, deals with material relevant to AusE, even if Australia had few concentrations of Scottish enclaves (mainly in Victoria). Here is a selection of features that Bauer considers as potentially deriving from ScotE that also occur in AusE; I add some comments where necessary:

- to want out 'to want to go out'; also available in varieties of AmE

- the phonetic distinction between which and witch, which is disappearing fast in both countries and even in $\mathrm{BrE}$

- a front articulation of /u/, which sounds [to a German's ear] like [ü]

- the retention of $[\mathrm{h}]$ in history, etc.

- the voiceless quality of word-final fricative sounds, as in bathe, similar to a process of devoicing in German; that has apparently not been commented upon at all

- the pronunciation of words like dance similar to AmE (with a sound like German [ä])

- the negation of sentences with never instead of not, e.g. 'I never done that'

- the use of $\operatorname{yous}(e)$ for second person plural

- the use of words like darg 'an amount of money for one's daily labour', littlie 'small children'

Bauer rightly has some reservations about whether all these features should be seen as ScotE in origin. Some of them do, as I have implied, occur in northern English and AmE, so they could have been taken to NZ and Australia in other ways than by Scottish immigrants. S. Butler is concerned with the selection of South-East Asian words for the Macquarie dictionary. She shows that Australian lexicography and AusE have well advanced in carving out an English-language region of their own by defining English as an Asian language that reflects Asian cultures and by locating Australia inside that region. That may well give them a market lead. M. Clyne looks at the problem 
of defining national identity in countries that rely on a pluricentric language. He retraces the main tenets of Australia's Labor policies on language and the 'sea-change' in Australia's self-perception. That, he suggests, is correlates with these factors, viz. the changed composition of the population; the influence of first generation migrants; and the need for reconciliation with Aboriginal Australians. His comments on the status and functions of BrE, mainstream AusE and ethnic varieties (in Australia) is informative and concludes that (to use my term) mAusE will be the national variety of the future and rest "on a nexus between language and nation and usually also on multiple identity" (1997:296). I leave out his remarks on NZE and on the role of German in Austria and Belgian Dutch. Closely related is G. Turner's paper on AusE as a national language. "To call Australian English a national language", he says (1997:339), "is to notice its double role. On the one hand it is a unifying force, bringing a collection of colonies together as a commonwealth, sinking differences (at least ideally); at the same time it is a distinguishing force, marking Australians off as culturally independent people, not transplanted Englishmen." And it is that function that highlights most the cultural values and the need to come to grips with a multicultural society.

Taking up a related theme, P. Peters and A. Delbridge look at the impact of H.W. Fowler's usage books on AusE, which they divide into influences in principle and influences in detail. As they aim to study the latter, they select a range of Fowler's topics and compare them with the BrE LancasterOslo-Bergen and the Australian Corpus of English. Having shown that there are significant departures between the two varieties, only some of which are consistent with Fowler's views, they turn to Australian usage guides. These guides increasingly depart from Fowler's legacy; Fowler fades into oblivion-but, surely, not only in Australia.

These remarks cannot and were not intended to do justice to the festschrift, but to show the liveliness of research on features of AusE, its national and international position, and the formative input from the past. One feature cannot go unnoticed, viz. the enormous amount of self-praise that is visible in Peters/Delbridge's paper. They comment on The Cambridge Australian English style guide, which was authored by P. Peters herself:

"She [P. Peters, GL] had an excellent grounding and achievement in the languages that have contributed most to the history of English... She has long had a fruitful research and teaching interest in the history of English orthography.... so there is no need for her to claim mere amateur status in this genre [of editing, GL]." (1997:315)

We linguists know her achievements, which extend to corpus linguistics and many other fields. But should an academic really write that of herself? We can wait to see her festschrift, can't we? 


\section{Siegel, Jeff, 2000. Processes of language contact. Studies from Australia and the South Pacific. Saint-Laurent (Canada): Fides, pp xvi+326. ISBN 2-7621-2098-5. Rezensiert von Gerhard Leitner, Freie Universität Berlin}

Language contact between English and other languages in Australia are increasingly attracting the interest of the wider international community, while in the past pidgins and creoles were not found in collections of articles or specialist studies (e.g. John Holm 1989. Pidgins and creoles. 2 vols. Cambridge: CUP). The collection of papers and maps in Wurm/Mühlhäusler/Tryon (Atlas of languages of intercultural communication in the Pacific, Asia, and the Americas, 1996, 3 vols. Berlin: Mouton de Gruyter), which was reviewed in Newsletter 12 (1998), paved the way for collections such as Siegel's. The book is not, of course, intended as a survey of Australia and the South Pacific (cf. Wurm/Mühlhäusler/Tryon 1996 in the Atlas); it is a collection conference papers and a few specially written ones that deal with (i) theoretical issues in the analysis of contact languages (J. Bresnan "Pidgin genesis and Optimality Theory"; T. Crowley "Simplicity, complexity, emblematicity and grammatical change"; J. Siegel's Introduction); (ii) case studies on the South Pacific (T. Crowley on Bislama, the creole in Vanuatu; J. Siegel et al. and C. Corne on Tayo in New Caledonia; C. Jourdan on the creole of the Solomon Islands; G. Smith on Tok Pisin in Papua New Guinea); and (iii) on language contact phenomena in Australia ( $\mathrm{H}$. Koch on the impact of Aboriginal languages on the grammar of pidgin English (pp 13-46); I. Malcolm on the linguistic (dis-) continuity of Aboriginal English from an early pidgin to today's dialect of English and possible remnants of the South Pacific pidgin (pp 123-144); J. Simpson on the role of Afghan cameleers in spreading pidgins and creoles (195-244); and J. Munro on the expansion of Kriol, the creole in the Northern Territory ( $\mathrm{pp} 245-270)$ ).

While all papers are worth a comment-especially the ones that touch upon theme (i) and areas closely connected with Australia, i.e. the Solomon Islands and Vanuatu-I will single out the Australian ones. The four contributions in question address different themes and I will embed them in the broad socio-historical and research context. In a paper entitled "The role of Australian Aboriginal languages in the formation of Australian pidgin grammar: transitive verbs and adjectives, Harold Koch studies two grammatical phenomena, (i) the marking of transitive verbs with a suffix (mainly -im, sometimes -it; e.g. givim or givit) and (ii) the use of pela (which is derived from English fellow or fella) as an adjective marker (Where big fellow water sitdown? 'Where is very big [i.e. the large body of] water located?'), amongst other uses. These features are typical of Australian pidgins, but have been attested all over the South Pacific. Two questions arise. The first is how their presence can be explained in terms of language contact, the second how their diffusion can be accounted for. Koch claims that Aboriginal languages acted as a substrate-with evidence from the languages close to the first settlement. Koch also holds that these languages led speakers to re-analyse English constructions like "John liked (th)em a lot" in terms of grammatical distinctions made in their own languages. And he adds that the new patterns became regular features of the NSW pidgin and spread across the continent, where they were learnt by new pidgin speakers. He fails to show is how they could spread from Australia to the South Pacific. He obviously accepts Wurm/Mühlhäusler/Tryon's (1996) hypothesis that Australia was a major player in the regional contact and that pidgins features were transported as a result of whaling, sealing, trepang fishing or sandalwood trade, but the details of the spread of these features remain untold.

Expanding on research done with Marek Koscielecki (Aboriginality and English, 1997, EdithCowan-University, Perth), Ian Malcolm's paper "Aboriginal English: from contact variety to social dialect" defines today's Aboriginal English (AborE) as a social dialect of English-he rejects the view that AborE is a 'dialect of Australian English'. He maintains that English was being "forced upon Aboriginal people from the beginning" and that it "was seen to be associated with a power which was progressively depriving Aboriginal people of their land..." (2000:125). The English of Aboriginal people was described at first as a "barbarous mixture of English with the Port Jackson dialect", which proves that it was a contact variety in origin. In his attempt to retraces the rise of today's AborE in the Sydney area, Malcolm contrasts what is known about the early contact variety 
with current speech samples from La Perouse. He concludes that AborE retains older features, especially regarding verb inflections; has features that reflect the on-going influence of non-standard AusE - though he tends to agree with the assumption that Aborigines have re-structured old patterns in novel (contemporary) ones and have discarded numerous older simplifications. Thus the transitive suffix -im, which was studied by Siegel, no longer turns up in AborE. "Aboriginal English has been drawing heavily on the intralingual resources of English as spoken in Australia", Malcom concludes (2000:140). Yet he believes in a continuity of the semantics of indigenous languages that manifests itself in discourse and text types. While one can agree with most of Malcolm's results, one should add that there has been a tradition of teaching English, a tradition which started with the famous Bennelong, who was kidnapped by Phillip as early as 1789 , and was continued in government and mission schools, as well as in many employments. There is, in other words, no bifurcation of the early jargon into a pidgin and creole line ( $c f$. Koch) and today's AborE—which would be necessary in his account but which he does not attempt to date.

Jennifer Munro's "Kriol on the move: a case of language spread and shift in Northern Australia", and Jane Simpson's "Camels as pidgin-careers: Afghan cameleers as a vector for the spread of features of Australian Aboriginal pidgins and creoles" deal with one of the two living creoles-the other being Torres Strait creole, spoken in the Torres Strait and the coast from Cape York to around Cairns. There has been a lengthy debate about whether Kriol originated in a single place, possibly Roper River (Ngukurr) on the south-eastern edge of Arnhem Land close to the Gulf of Carpentaria, or whether it arose independently in several places. The most favoured place would be Barunga in the Kimberley. Multiple creolization and convergence or single creolization and language shift is not only an problem of historical evidence; it is also one of theory. Arguing for the view that Kriol originated in Roper and was adopted elsewhere, Munro looks at socio-historical, linguistic and socio-linguistic evidence. She argues that there are seven dialects between the Barkly Tablelands south of the Gulf of Carpentaria to Katherine River, close to Derby in the Kimberly and Daly River southwest of Darwin. She provides a lucid account of the contact situations in the Roper River Mission, Daly River army camps and the Moola Bulla Native Welfare camp near Halls Creek. As only Roper River fulfilled the conditions for the rise of a creole, she believes one must search elsewhere for evidence to explain today's dialect situation. That she finds in the expansion of the pastoralist industry that reached the Northern Territory by 1870 and the Kimberley by the 1880s. The army camps that were set up in several places in the Northern Territory in the first part of the 19th century generated a high level of mobility and policies of assimilationism and government intervention in the 1950s and 1960s added to inter-Aboriginal contact. These developments, then, made it possible for speakers of different languages to shift to the already available Kriol or its precursors, the Northern Territory pidgin. As groups started to identify with their new habitations, they acentuated dialectal peculiarities and incorporated features from AusE. While this account is interesting, her data base - she has one half hour from the Daly River creole-is far too small to permit such general conclusions, it would seem. Her paper is more hypothetical than factual.

It is interesting that Munro did not consider the role of Afghan camel drovers-the theme in Simpson's paper. There has been little work on Afghans and Moslems in the 19th century (e.g. Mary L. Jones, An Australian pilgrimage, 1993, Melbourne: Victoria Press), so that Simpson would deserve attention. She re-iterates the period of Afghan immigration between the 1860s and 1900, their involvement in exploring expeditions, transport, construction, and the relations with Europeans and Aborigines. Her survey of Afghan English shows that it contained typical pidgin features, such as the transitive marker -im (cf. Koch), and adds an extensive appendix of Afghans pidgin English. She draws on an enormous range of historical data, which are worth knowing, but fails to show details of how Afghans adopted and spread Aboriginal pidgin.

Critical remarks aside, the book is an excellent contribution to contact languages in Australia, their relationship to the South Pacific pidgins and creoles and to the intriguing theoretical issues they give rise to. It is a useful, up-to-date book for the language side of Australian Studies. 
Fishman, Joshua, ed., 2001. Can threatened languages be saved? Reversing language shift, revisited. A 21st century perspective. Clevedon, UK: Multilingual Matters, pp xvi+503. ISBN 185359-493-8 (hbk), 1-85359-492-X (pbk). Rezensiert von Gerhard Leitner, Freie Universität Berlin

Fishman's career in the sociology of languages spans several decades. He has shaped and created several disciplines such as language and ethnicity or language and nationalism. Reversing language shift, revisited (RLS) is a sequel to his Reversing language shift; theoretical and empirical foundations of assistance to threatened languages (1991, Benjamins), in which he proposed a theoretical model that would predict the likely success or failure of language maintenance efforts. RLS theory "being more than merely a descriptive tool, attempts to locate the functional disruption of X [some language, GL] in social space and has suggested a widely (...) useful series of stages for doing so. Such location helps establish both focus and priorities for RLS efforts, rather than merely presenting a redundant restatement of Xish's functional, institutional or attitudinal deficiencies" (his emph.; 2001:466f). The so-called Graded Intergenerational Disruption Scale (GIDS)—a set of criteria that measures the degree of disruption of the maintenance of a language and the efforts that have been expended to maintain it—is restated on $\mathrm{p} 466$ for those unfamiliar with the theory.

Fishman introduces into the theme of why it is so hard to save threatened languages (pp 1-22) and summarizes what is contained in the body of the book ( $\mathrm{pp} \mathrm{451-483).} \mathrm{There} \mathrm{are} \mathrm{a} \mathrm{list} \mathrm{of} \mathrm{contributors,}$ a brief introduction and a detailed index. The 17 focal papers deal with (i) languages in the Americas, i.e. Navajo, Puerto Rican Spanish (in New York), Yiddish, Atom (in Mexico), French (in Quebec), and Quechua; (ii) in Europe, i.e. Irish, Frisian, Basque, and Catalan; (iii) in Oko in Africa, Andamanese in India, Ainu in Japan and Modern Hebrew; (iv) immigrant and indigenous languages in Australia (M. Clyne; Lo Bianco/Rhydwen), and Maori in New Zealand (R. Benton/N. Benton). With a few additions-e.g. Australia now has two articles- these languages had been discussed in 1991 so that their situation could now be compared with that in 2001. The relevance of this collection for Australianists lies in three aspects, for one, the coverage of Australia's LanguagesOther-Than-English; secondly, the theoretical perspective provided by RSL and, thirdly, the fact that LOTEs can be compared with others elsewhere. I will focus on aspects relevant to Australianists.

GIDS is the guiding principle for all contributions and it is pertinent to mention some of its features. RLS, Fishman says "is the linguistic part of the pursuit of ethnocultural self-regulation which democracies and international bodies are increasingly recognising as a basic right for indigenous (and often also immigrant) populations." (2001:452). Human linguistic rights is the underlying theme and it is important to understand the mechanisms of support and danger, as well as their interrelations. The eight criteria divide into two sets, four of which have to do with what language communities do, four deal with steps in the wider community. Fishman emphasizes that there can be no progress if speakers or the remaining speakers do not take on an active part, even to the point of relearning the language so that they can act as interlocutors (not to mention the word models). There must be a self-contained cultural network in which children are exposed to and motivated to learn the language. If the "intergenerational and demographically concentrated home-familyneighbourhood" network is not functional, there can barely be progress that would lead the mainstream society to construct (or permit) pockets of language transmission for community uses. That sounds plausible, but the fact is that many RLS efforts start with those measures, hoping language communities would react positively. A top-down approach, however, does not work. On the other hand, many language communities are so widely dispersed that such a network is inconceivable in the first place and, if anything, top-down measures are seen to assist a little.

What is worth emphasizing is Fishman's humanistic approach-despite his style that is imbued with jargon (e.g. mainstream languages are called "X", speakers "Xians", other languages are "Y", speakers "Yians", there are "Xish" interests, etc.). He brings out clearly the culture-language-speaker nexus that is so often overlooked in general linguistics. Thus he says "[I]t is the specificity of the 
linguistic bond of most cultural doings that makes the very notion of a 'translated culture' so inauthentic and even abhorrent to most ethnocultural aggregates." (2001:3). Aboriginal cultures are cases in point, since languages are considered a repository of knowledge. Immigrant cultures vary considerably, as Smolicz has repeatedly shown in his memoir studies where speakers of a language report extensively what role they assign to their native language.

Clyne's contribution is an up-to-date survey of the migrant LOTEs situation in relation to the shift of first generations to English, the home-family-neighbourhood network regarding German and other LOTEs, library holdings in LOTEs, radio and TV broadcasting, newspapers and periodicals in LOTEs, etc. He adds that it would be useful to include in future studies the role of the internet, which provides access to a language's native land. Following step-by-step the GIDS model, he shows that Australia has missed a chance. Instead of progressing towards LOTE maintenance, at least, at the level of education for its international needs, it has demolished the necessary infrastructure in tertiary education for the sake of economic rationalism and privatization. Lo Bianco/Rhydwen's paper highlights the fact that "[T]he social structures of indigenous speech communities are dramatically unlike those of the wider society, nor are they similar to those of other minority speech communities that have entered westernised mainstream society." (2001:392). They argue for modifications to GIDS to account for them and for the absolute necessity to keep the domains in which indigenous languages might be used separate. They make the point that in some northern areas RLS may strengthen an indigenous language at the expense of others, as speakers turn to using it as a lingua franca. They also mention the negative impact of economy-driven considerations, even though some languages have experienced modest ups during the past ten years. But: "[D]espite occasional instances of revival that attain short-term, unexpected and spiritually uplifting gains for communities of speakers of traditional languages the pattern of attrition and extinction appears inexorable." (2001:419). Australia, thus, does not provide a show case for other countries. Both papers focus on the negative impact that the shift of language policies towards economic considerations has had. These policies have done away with what is necessary in secondary and tertiary education so that it is no longer possible to train the number of teachers and get quality in teacher training that would have been necessary for LOTE teaching to expand. What is worse is the cessation of funding of bilingual programs in indigenous languages in the Northern Territory is, as Lo Bianco/Rhydwen argue, a particular blow.

The book provides a stimulating theoretical perspective on RSL and a number of pertinent case studies. It offers input for political and social scientists who take an interest in the interaction and self-centred aspirations of ethnic groups. Language is but one small aspect of social politics but has repercussions in media and educational policies, public sector funding in out-of-school education (e.g. public libraries), etc. The Australianist will find important information on Australia. 
Simpson, Jane, David Nash, Mary Laughren, Peter Austin, Barry Alpher, eds, 2001. Forty years on: Ken Hale and Australian languages. Pacific Linguistics, Research School of Pacific and Asian Studies, The Australian National University, Canberra, pp xviii+528. ISBN 08583 524X.

\section{Rezensiert von Gerhard Leitner, Freie Universität Berlin}

Ken Hale, an American linguist and pioneer in the study of Aboriginal languages, began his work in Central Australia in 1959. Over four decades he has worked on scores of languages, has written grammatical, phonological, lexical, semantic and other analyses. He collected a large number of tape recordings, transcriptions, acted as advisor of the Federal government on bilingual education programs, which led to such schools in the Northern Territory, etc. This book is devoted to the impact of his work and contains 36 papers written by linguists from Australia, the USA, Canada and Denmark. There is a bibliography of Hale's work and an index of the languages covered. As the book is dedicated to Hale, the articles are mainly about the languages that he worked on, i.e. the Arrernte, Warlpiri and Arandic families, which are spoken in the NT, Qld, SA and WA.

Many papers deal with specific features of the phonology, lexis, syntax and semantics of the languages in question. Others are autobiographical and deal with Hale's impact on changing attitudes to Aboriginal Australians. To mention the reminiscences of his wife on their arrival in Alice Springs in 1959 and Hale's notes on his strained relations with T.G.H. Strehlow, the German linguist-missionary in Hermannsburg, which have been edited by J. Green. S. Wurm, another leader in the field, recollects his first meeting with Hale; M. Sharpe writes about his impact on the rise of the field of Australian Linguistics and his association with Capell, a Church of England bishopanthropologist-linguist in Sydney. Other papers deal with songs (Alpher/Keeffe) and literature (Cataldi). A few papers focus on educational issues, such as bilingual education (Hoogenraad), the School of Australian Linguistics with its campuses in Batchelor (south of Darwin) and Alice Springs (Shapre; Black/Breen). That institution has done much to train Aboriginal teacher-linguists and to make languages accessible. Inspired by Hale, amongst others, it has helped to promote Aboriginal Language and Culture centres that span the country and assist in language and culture maintenance. Other papers focus on lexico-cultural matters of interest to those concerned with the language-culture dimension. Laughren discusses the avoidance style that is used in kin relations. Many papers embed their problems in comparative-typological linguistics (e.g. Koch, Pensalfini).

It is impossible to do justice to the welter of information that this book contains. With its coverage, detail and approaches, it is mainly for theoretical and applied linguists with an interest in Australian languages and for those in the field of the history of language studies, esp. the rise of Australian Linguistics as a discipline and today's Aboriginal networks that promote language studies and language teaching at school. The book contains information that benefits ethnologists, anthropologists and educational theorists. As for Australian Studies, it will benefit those who include a linguistic component. (Related collections of papers are M. Walsh, C. Yallop, eds, 1993. Language and culture in Aboriginal Australia and Wurm/Mühlhäusler/Tryon, eds, 1996, Atlas of languages of intercultural communication in the Pacific [etc.]. Both have been reviewed here.) 


\section{Blair, David, Peter Collins, eds, 2001. English in Australia (= Varieties of English Around the World, G 26). Amsterdam: Benjamins. 366S., ISBN 9027248842. Rezensiert von Clemens Fritz, Freie Universität Berlin}

Blair und Collins English in Australia (EiA), langerwartet und auch überfällig, bildet einen wertvollen Beitrag zum Australian English (AusE). Jüngere Erscheinungen, z.B. Seal, Graham, 1999, The lingo. Listening to Australian English, und Burridge, Kate, Jean Mulder, 1998, English in Australia and New Zealand. (s. GASt Newsletter 1999) enthalten, trotz verheißungsvoller Titel, kaum Neues. Erstere schließt sich der essayistischen Tradition von Bakers The Australian language an und zweitere präsentiert eine eher traditionelle Grammatik, die mit dem Titel des Buches wenig zu tun hat. In letzter Zeit schienen sogar die Publikationen zum New Zealand English (NZE) denen des AusE den Rang abzulaufen, was sich u.a. anhand von Bell und Kuiper (2000) zeigt. Umso wichtiger ist das Erscheinen des vorliegenden Buches, das den Stand der Forschung zum AusE abbildet und neue Erkenntnisse beinhaltet.

EiA ist der zweite Sammelband über das Englische in Australien der beiden Herausgeber. Der erste erschien 1989 unter dem Titel Australian English - The language of a new society. Der Vergleich beider Titel zeigt eine wichtige Akzentverschiebung, welche die Beschäftigung mit dem AusE genommen hat. Ging es 1989 noch um die Feststellung, dass das AusE eine eigenständige Varietät sei, ist die Zielsetzung nun eine andere. Nicht der Vergleich des AusE mit anderen Varietäten steht im Vordergrund, sondern die Variabilität des Englischen innerhalb Australiens, wie sie u.a. im Aboriginal English (AborE) und den verschiedenen ethnic varieties zum Ausdruck kommt.

Das Buch mit 19 Beiträgen ist in zwei Sektionen unterteilt. In der ersten sind Aufsätze über die Strukturen des AusE (unterteilt in die Bereiche Phonologie, Morphologie, Syntax und Lexikon) zusammengefasst, die zweite befasst sich mit der Variation des Englischen in Australien (Torres Strait und AborE, ethnic Varieties, diachrone und altersabhängige Variation sowie regionale Unterschiede). Die Einleitung der Herausgeber, Language and identity in Australia, zeichnet eine Entwicklungs- und Forschungsgeschichte des AusE. Diese gründet auf der Überzeugung, dass die sprachliche und die politisch-soziale Findung einer nationalen Identität in Australien Hand in Hand gehen. Das Ringen um Identität, verstanden als Abgrenzung nach außen, zeigt sich in der diachronen Entwicklung der Aussprache und Lexik. Nach Erreichen nationaler Selbstsicherheit ist es möglich, die Identität im Inneren zu erweitern, sich als multikulturelle Gesellschaft zu verstehen.

"Any survey of the status and nature of English in Australia will reveal how closely the national language, in its strength and in its variability, reflect the essential cohesivness and diversity of the home culture. [...] As the language has developed over 200 years, its lexicon has continued to expand to serve the needs of a developed southern hemisphere nation, and its varieties have continued to reflect the multi-ethnic and multi-cultural nature of Australian society." (S. 11)

Die ersten drei Beiträge widmen sich der Phonologie. Cox und Palethorpe untersuchen dabei das klassische Feld der Vokale im AusE (Vowel change: synchronic and diachronic evidence), während sich Tollfree (Variation and change in Australian consonants: reduction of / $/ \mathrm{t} /$ ) und Borowsky (The vocalisation of dark $l$ in Australian English) dem weniger untersuchten Gebiet der Konsonanten nähern. Cox und Palethorpe stellen sich der Aufgabe, die Ergebnisse der Standardwerke (vgl. Mitchell/Delbridge, 1965, The pronunciation of English in Australia) auf ihre Gültigkeit für die Gegenwart zu untersuchen. Dazu gehen die Autoren verschiedenen Ansätzen nach. Zum einen die Untersuchung der Replizierbarkeit der Ergebnisse im real-time Verfahren (hierbei werden äquivalente Daten von nach gleichen Kriterien ausgesuchten Gruppen untersucht, wobei zwischen den Erhebungen eine Zeitspanne liegt), zum anderen im apparent-time Verfahren (die Daten kommen aus derselben Zeit, werden jedoch von unterschiedlichen Altersgruppen erhoben). Beide Vorgehensweisen zielen darauf ab, herauszufinden, ob ein Sprachwandel feststellbar ist. Die realtime Analyse zeigt, dass es in den letzten 30 Jahren im AusE zum Wandel in der Aussprache der Vokale gekommen ist. Auch die apparent-time Methode erweist dies. Es zeigen sich weitere Unterschiede, die auf kontextuelle Unterschiedlichkeit sowie auf Unterschiede im Alter und regionaler Herkunft der Untersuchten zurückzuführen sein könnten. Erstaunlicherweise wird dem 
letzten Kriterium der größte verzerrende Effekt zugemessen, was herkömmlichen Vorstellungen über das AusE widerspricht. Tollfree untersucht Varianten von /t/, nämlich eine frikative, eine glottalisierte und eine tapped (d.h. mit Zungenschlag) Realisierung, die ihrer Meinung nach neuere Entwicklungen widerspiegeln. Eine auditive und akustische Analyse ihrer Daten (Interviews) zeigt eine hohe Zahl von reduzierten Varianten, wobei die tapped Form bevorzugt wird.

Der Artikel von Borowsky ist, wie der vorhergehende eher eine allgemeine phonetische Studie ohne besonderen Bezug zu Eigenheiten des AusE. Der Autor sucht herauszufinden, in welchen phonetischen Kontexten ein 'dark l' vokalisiert werden kann und baut dabei auf eine frühere Studie mit Horvath (1997) auf. Zunächst standen nur Daten aus Adelaide zur Verfügung, das nun durch Aufnahmen aus anderen großen Städten ergänzt wurde. Eine Abweichung hat sich in den Ergebnissen nicht ergeben, was darauf hinweist, dass diese Art von Vokalisierung ein allgemeines, nicht regional begrenztes Phänomen ist. Allgemein unterscheidet Borowsky zwischen Positionen, die eine konsonantische und solchen, die eine vokalische Realisierung nahe legen. Die Daten wurden mit Hilfe des Programms VARBRUL statistisch ausgewertet. Danach ist eine Vokalisierung am wahrscheinlichsten, wenn das /1/ am Ende einer Silbe Teil eines Konsonantenclusters ist (z.B. milk). Auch fördern hohe Vokale wie /i/ und / $\mathrm{d} /$ und eine nukleare Position in der Silbe die Vokalisierung.

Die einzigen Beiträge zu den Themen Morphologie und Syntax liefern Simpson (Hypocoristics of place-names in Australian English) und Newbrook (Syntactic features and norms in Australian English). Simpson verwendet hypocoristic als Überbegriff für sämtlich mögliche Formveränderungen von Namen (so z.B. Kürzung von Brisbane $=>$ Briz, und Suffigierung von Goondiwindi $=>$ Gundy). Sie stellt die Frage, ob es Veränderungen gibt, die für das AusE typisch sind. Ihre Forschungen ergeben, dass die Art der Veränderung mit der Einstellung des Sprechers in Verbindung steht und dass sie von der Lautumgebung beeinflusst wird. Definitive Antworten vermag sie jedoch nicht zu geben und verweist daher auf zukünftige noch intensivere Studien.

Sehr interessant ist der Artikel von Newbrook zur Syntax des AusE. Dieser beginnt mit der Feststellung, dass sich das AusE hinsichtlich seiner Syntax nicht wesentlich von anderen Varietäten des Englischen unterscheidet, bietet aber dann doch einen sehr lesenswerten und informierten Katalog über verschiedenste Besonderheiten. Seine Ergebnisse gründet er auf Untersuchungen des Australian Corpus of English und auf die Lektüre mehrerer style guides, die auf dem australischen Markt anzutreffen sind. Wissenswert ist auch Folgendes:

[...] it is clear [...] that most syntactic features typical of formal styles which are in fact characteristically Australian (at least statistically) are not generally recognised as such by Australians. Surprise is often expressed at the information that some such feature is rare or absent, or would be deemed non-standard, in (especially) BrE. In consequence, the standard variety of AusE, in respect of syntax, currently constitutes more of a de facto than a de iure set of norms; [...]. [S. 114f]

Spezifisch für das AusE sind: die Möglichkeit des Singular und des Pluralkonkords bei Sportteams (North Melbourne is und North Melbourne are), die Verbindung von same mit einem Possessivpronomen in der Bedeutung 'die selbe' (Can I keep my same phone number?), die geringere Häufigkeit der Zeitverschiebung in der indirekten Rede, die Verwendung des Superlativs mit since in der Bedeutung, dass ein neuer Rekord einen alten ausgelöscht hat (His score of 200 was his highest since he made 175 in 1995.) und eine Umkehrung der üblichen Kommasetzung für restriktive und nicht-restriktive Relativsätze. Newbrook findet auch Übereinstimmungen des AusE mit dem American English, darunter die epistemische Verwendung des Modalverbs have (got) to (anstatt must) und die Einsetzung von would bzw. would have + Partizip Perfekt im if-clause (If they would do that it would be better und nicht If they did that it would be better).

Die letzten drei Artikel des A-Teils des Buches nehmen alle die Lexik des AusE ins Visier. Moore (Australian English and indigenous voices) untersucht dabei den Einfluss, den das Verhältnis von Aborigines und weißen Einwanderern auf das AusE hat. Er stellt dabei eine Zunahme von Wörtern aus den Sprachen der Eingeborenen fest. Diese Worte werden aus zwei verschiedenen Quellen 
gespeist. Zum einen werden neue Tier- und Pflanzenarten mit Wörtern aus Aboriginesprachen gebildet, es werden Orte mit europäischen Namen umbenannt und Bezeichnungen aus der Kultur der Aborigines nehmen im AusE zu (z.B. dreamtime oder secret women's business). Zum anderen wird auch ein neues Vokabular geschaffen, das es ermöglicht, den Prozess der Emanzipation der Aborigines darzustellen und nachzuvollziehen (land rights, Sorry Day). Insgesamt ergibt sich so die Möglichkeit, der Multikulturalität Australiens größeren sprachlichen Ausdruck zu verleihen.

In den nächsten beiden Beiträgen findet sich kaum Neues, sie sind vielmehr Forschungsberichten. So greift Butler (Australian English - an identity crisis) die Frage auf, ob das AusE durch den Einfluss des AmE vom Aussterben bedroht sei. Dies ist natürlich nicht der Fall, denn die Lexik des AusE ist in vielen Fällen direkt mit der Geschichte und Kultur Australiens verbunden, so dass eine vollständige Amerikanisierung kaum möglich erscheint. Nur bestimmte Lexeme werden übernommen, die sich dem australischen Kontext anpassen.

Die Untersuchung der Varietäten des Englischen wird zunehmend von dem Vorhandensein von Korpora geprägt. Peters (Corpus evidence on Australian style and usage) referiert kurz über die Entwicklung von Korpora in Australien. Als Ergebnisse korpusorientierter Arbeit haben sich für das AusE die vermehrte Verwendung der Mandativen Sprechabsicht des subjunctive, die vermehrte Verwendung von Kontraktionen und von get-Konstruktionen im Passiv herausgestellt.

Der zweite Teil des Buches stellt nun gerade die Variabilität des AusE in den Vordergrund und nicht die sinnstiftende Abgrenzung nach außen. Shnukal beginnt mit der Darstellung des Torres Strait English, des Englischen der zweiten Gruppe eingeborener Australier, die in Queensland leben und aus Papua Neu Guinea stammen. Die meisten sprechen eine Kreolsprache, das Torres Strait Creole, das doch nicht auf dem Englischen basiert, wie bis vor kurzem noch geglaubt wurde. Es gibt vier lebende Sprachen in dieser Gemeinschaft, die zwei eingeborenen Sprachen der beiden ursprünglichen Volksgruppen, die Kreolsprache und das Englische in seiner Torres Strait Variante. Ziel Shnukals ist es, eine erste Beschreibung des Torres Strait English (TSE) zu liefern und es in Beziehung zum mainstream AusE (mAusE) zu setzen. Es wird deutlich, dass sich das TSE phonologisch, phonetisch, morphologisch, syntaktisch und auch lexikalisch vom mAusE absetzt, was in der dortigen Sprachsituation seinen Ursprung hat.

Das Aboriginal English (AbE) wird von Malcolm (Aboriginal English: adopted code of a surviving culture) vorgestellt. Auch er weist keine neueren Forschungen vor, sondern resümiert den aktuellen Forschungsstand, dies allerdings mit sehr viel Klarheit und Wissen. Die ersten Abschnitte widmen sich der Geschichte des Sprachkontaktes zwischen den Aborigines und den weißen Einwanderern, sowie den interkulturellen Missverständnissen sprachlicher und sozialer Art. Interessant ist dabei, dass sich im AbE eine soziolinguistische Kontinuität an kommunikativen Verhaltensweisen finden lässt, die noch der vorenglischen Periode entstammen. Viele Aborigines sehen sich in einem bikulturellen Dilemma gefangen. Die eine Kultur gibt ihnen Würde und Identität, während die andere sie dieser Dinge beraubt, sie dafür aber ernährt. Das AbE nahm wohl den Weg von Pidginisierung über eine Kreolphase bis hin zur Dekreolisierung in die heutige Form. Dabei wanderte das New South Wales Pidgin English mit den europäischen Siedlern mit und gelangte so fast überall in Australien zur Anwendung.

Clyne, Eisikovits und Tollfree (Ethnic varieties of Australian English) zeigen die Andersartigkeit von ethnisch beeinflussten Varianten des AusE, ein Forschungsgebiet, das noch relativ jung ist. Die untersuchten Ethnien sind dabei die klassischen älteren Einwanderergruppen wie Deutsche, Griechen und osteuropäische Juden. Immerhin sprechen heutzutage 14,6\% der Bevölkerung Australiens zuhause eine Sprache, die nicht Englisch ist. Je nach Gesprächssituation und Auswahl der Informanten (Sprecher der ersten, zweiten oder dritten Generation) kommen unterschiedliche Ausprägungen der Ethnolekte zur Geltung.

Im Gegensatz zu den vorgenannten Autoren, wendet sich Kiesling (Australian English and recent migrant groups) z.T. neueren Einwanderergruppen (d.h. nach Aufhebung der White Australia Policy in den 1970ern) zu. Dazu gehören vor allem solche aus dem Mittleren Osten und aus Südostasien. Er möchte untersuchen, ob in diesen Gruppen eine homogenisierende oder eine divergierende 
Entwicklung hinsichtlich der Aussprache der Vokale vorherrscht. Sein Untersuchungsgebiet sind drei Stadtteile Sydneys, die alle einen Immigrantenanteil von über 50\% aufweisen. Als Ziel einer eventuellen Homogenisierung bzw. Divergierung werden die klassischen Soziolekte des AusE Broad, General und Cultivated genannt (vgl. Mitchell und Delbridge 1965). Der Autor zeigt, dass es eine statistisch signifikante Korrelation zwischen dem Geschlecht und dem ethnischen Hintergrund und der Ausprägung der Vokale gibt. Danach kann bei Frauen nicht von einer Annäherung an die Standardaussprache der Vokale gesprochen werden, die Daten für die Männer sind jedoch nicht eindeutig. Als mögliche Gründe dafür werden soziale Netzwerke, die unterschiedliche Akkulturation von Männern und Frauen und die Vitalität der jeweiligen Ausgangssprache genannt.

Die Darstellung und Untersuchung diachroner Entwicklungen ist die Sammelüberschrift für die nächsten fünf Artikel. Einen eher schwachen Beitrag liefert Curtain (The acquisition of colloquialisms by non-native speakers). Sie testete die Fähigkeit von Australiern und Studenten aus Malaysia, traditionelle (aus dem AusE kommende) und moderne (aus dem AmE kommende) Kolloquialismen zu erkennen. Wenig überraschend sind die Ergebnisse. Für die Australier sind die Kenntnisse der Wendungen ans Lebensalter gebunden, während sie bei den malaysischen Studenten mit der Länge des Aufenthalts in Australien korrelieren.

Auch Bradley und Bradley (Changing attitudes to Australian English) haben wenig Überraschendes $\mathrm{zu}$ bieten. Sie konzentrieren sich darauf, einen Wandel in der Einstellung zum AusE darzustellen. Für historische Einstellungen verweisen sie auf Autobiographien, Zeitungskolumnen und Leserbriefe, Film und Fernsehen sowie auf populärwissenschaftliche Bücher über die Lexis des AusE. Eine eigene Untersuchung liefern sie mit Interviews mit Informanten aus Melbourne, die sie 1980 und 1995 durchgeführt haben (dabei konnten 199527 der 40 ursprünglich Interviewten wieder befragt werden). Daraus kann entnommen werden, dass sich die Akzeptanz des AusE vergrößert hat, wobei Jüngere dabei Vorreiter sind. Ein wenig informativer ist der Subjective Reaction Test (SRT), bei dem Sprecher der verschiedenen Soziolekte des AusE hinsichtlich ihres Status und ihrer Freundlichkeit/Ehrlichkeit (F/E) eingeordnet werden sollten. Das Ergebnis war, dass Broad AusE mit sehr geringem Status und nur mittlerer F/E assoziiert wurde. Cultivated hatte die höchsten Ergebnisse für Status und die schlechtesten für F/E, während General für ersteres ein mittleres und für zweiteres das beste Ergebnis aufwies. Daraus kann gefolgert werden, dass General die akzeptierteste Variante des AusE ist, was nicht wirklich neues Wissen darstellt.

Sehr interessant und auch kontrovers ist hingegen der Artikel von Yallop (A.G. Mitchell and the development of Australian pronunciation), der u.a. auch eine Laudatio auf den 1997 verstorbenen großen Forscher darstellt. Mitchell hatte sich in seinen letzten Jahren nochmals darauf konzentriert, Licht ins Dunkel der Entwicklung der Soziolekte des AusE zu bringen und ging dabei historisch genauer und systematischer vor als bisher üblich. Da er sein Werk nicht mehr selbst beenden konnte, haben es nun Yallop und andere unternommen, Mitchells Thesen zu publizieren. Danach entwickelte sich das Broad AusE im Rahmen der von Sträflingen dominierten Sprachgemeinschaft bis zum Jahre 1830. Dabei homogenisierten die Neuankömmlinge ihre unterschiedlichen Dialekte, während die Kinder die einzige weiter verbreitete Variante annahmen, nämlich eine nivellierte Form des Englischen mit London als Ursprung. Das General AusE entstand in Victoria im Zuge des Goldrausches (ab den 1850ern), als Australien von Neuankömmlingen überschwemmt wurde. Dabei wurde es als städtische und prestigeträchtige Form vom Broad differenziert. Das Cultivated setzen Mitchell/Yallop als eine späte Neuerung an. Das Received Pronunciation (RP) als gültiger Aussprachestandard gelangte erst Ende des 19. Jhs. in England zur Geltung. Danach beginnt eine zunehmende Abwertung bis dahin akzeptierter Aussprachevarianten (regional nicht sozial), die in Australien zur "white-hot indignation of the 1930s and 1940s" (S. 300) und für viele Australier zu einer Annäherung an das RP führte. Diese Theorie setzt sich deutlich von bisherigen Erklärungsversuchen der Entwicklung der Soziolekte des AusE ab, steht aber auf sicheren historischen Fundamenten. Ob sie sich durchsetzen wird bleibt abzuwarten.

Delbridge (Lexicography and national identity: the Australian experience) stellt einen Zusammenhang zwischen der zunehmenden Akzeptanz des AusE als eines Standards und der 
Kodifizierung dieser Varietät her. Dazu erläutert er die Leitlinien der wichtigsten Wörterbücher für das AusE. The Australian National Dictionary (Ramson 1988) folgt der Tradition des Oxford English Dictionary und bietet in 6.000 Haupteinträgen Chronologien und Definitionen von Australianismen an. Damit ist es ein wichtiges Werkzeug für Literaturwissenschaftler, Historiker und andere historisch Interessierte. The Macquarie Dictionary (Delbridge et al. 1981/1997) schließt diese Lücke, indem es in 100.000 Haupteinträgen (darunter 20.000 enzyklopädische) ein umfassendes Referenzwerk bietet. Dieses wurde mittlerweile von der Australian Broadcasting Commission, der Australian Education Commission, anderen offiziellen Organen und auch den Bürgern Australiens als Standardwerk akzeptiert. Auch jährliche Style Councils und Style Guides etablieren einen Standard des AusE. So ist die Kodifizierung des AusE nicht nur Ausdruck einer zunehmenden Akzeptanz dieser Varietät, sondern trägt auch zur ihrer Akzeptabilität bei.

Australian English in interaction with other Englishes ist der Titel des Artikels von Taylor. Dieser baut auf seinen früheren Untersuchungen auf und zeigt die Geschichte der Verknüpfung des AusE mit anderen Varietäten des Englischen. Dabei beginnt er mit der Formierung des AusE als Konsequenz einer Vermischung von Formen des Englischen in den frühen Jahren der europäischen Kolonisierung, wobei er dem IrE mehr an Bedeutung zumisst, als dies gemeinhin getan wird. Das AmE beeinflusst das AusE in wenigen Ausdrücken vor 1850, dann stärker zur Zeit des Goldrausches in Australien und seit dem 2. Weltkrieg sehr stark über Filme und die Computerindustrie. Auch gegenüber dem BrE stellt sich das AusE vornehmlich als empfangende Varietät dar, wenn es auch neuerdings über populäre daily soaps wie Neighbours Teile der britischen Bevölkerung in Lexis und Aussprache zu beeinflussen scheint. Das NZE ist historisch und geographisch bedingt dem AusE sehr ähnlich und hat auch zu verschiedenen Zeiten stark unter dem Einfluss des AusE gestanden. Ein Einfluss in der anderen Richtung konnte bisher noch nicht überzeugend nachgewiesen werden.

Der letzte Beitrag des Buches unter der Überschrift regionaler Variation im AusE stammt von Horvath und Horvath (A geolinguistics of short A in Australian English). Darin werden die unterschiedlichen Aussprachevarianten von Wörtern wie dance, answer, etc. innerhalb Australiens untersucht. Geolinguistik unterscheidet sich von der traditionellen Dialektgeographie in den Methoden der Datenerhebung und -auswertung, wie Horvath und Horvath überzeugend zeigen. Die Autoren vergleichen in Sydney erhobenes Material mit den Informationen aus dem Macquarie Dictionary und stellen fest, dass die Beschreibungen des Wörterbuches größtenteils akkurat sind. Im Vergleich mit Daten aus anderen australischen Großstädten stellt sich jedoch heraus, dass die selben Worte durchaus unterschiedlich ausgesprochen werden. Eine statistische Analyse mit Hilfe des Programms VARBRUL filtert heraus, dass Geschlecht und Alter der Informanten keine Hinweise auf die Aussprache ergeben. Jedoch sind der Ort, das Wort selbst und in geringem Maße auch die soziale Zugehörigkeit des Sprechers wichtige Bestimmungsfaktoren für die Wahl zwischen Short A und Broad A. Diese Herangehensweise an regionale Differenzierung im AusE ist noch sehr jung und verspricht noch weitere interessante Untersuchungsergebnisse.

Insgesamt finden sich in dem Buch interessante Forschungen. Die einzelnen Beiträge sind dabei aber durchaus von unterschiedlicher Qualität und Aktualität. Leider fehlt eine zusammenfassende Bibliographie (was aber von Taylor, Leitner, Fritz, i.Arb., behoben werden wird) und nähere Angaben zu den Autoren, z.B. E-Mail Adressen oder Homepages der Institute. Trotzdem darf das Buch auf keinem Fall in dem Bücherschrank eines Australianisten fehlen. 
Ricento, Thomas, ed., 2000. Ideology, politics and language policies. Focus on English. Amsterdam: Benjamins, pp x+197. ISBN 9027218366 (Eur; hb), 27218374 (Eur; pb). Rezensiert von Gerhard Leitner, Freie Universität Berlin

Ideology, language policy, the spread of English and the allocation of status and resources languages other than English in anglophone countries is a theme that is attracting considerable interest. Ricento's collection of papers, which derives from two conferences held in the USA in 1997-8, is a case in point. It contains case studies on Australia (Helen Moore), the USA (T. Wiley), North India (S. Sonntag), South Africa (S. Ridge) and Sri Lanka (S. Canagarajah) and a few theoretical and methodological papers, such as Ricento's overview over language policy and planning, A. Pennycook's historical survey of the ideological lessions to be drawn from the spread of English in former colonies and R. Phillipson's discussion of the role of English in creating a new 'world order'. The book brings together a distinguished set of authors, who broadly agree on the fact that the spread of English calls for a critical political discussion, that English has ideological implications and affects human rights globally. Policy and planning need be discussed within a wide societal spectrum which identifies the power centres, the elites and the dominated. Politically, the authors adhere to modern left-wingism and feminism that contrasts sharply with traditional anglicists' views like those of D. Crystal (English as a global language, 1997). Having said that, I must add that these are refreshing views that permit a critical approach to policies in Australia, a nation that has often been taken as case that Europe could emulate.

Though Australia and its language policies are mentioned in several papers, Helen Moore's "Language policies as virtual realities: two Australian examples" (pp 29-47) is the centrepiece. She was active in the lobby groups that promoted language policies in Australia and presents a somewhat personalized account of the shift from the first explicit policy, National Policy on Languages (NLP; Lo Bianco 1987), to the second one, Australia's Language: The Australian Language and Literacy Policy (ALLP; Department of Education, Employment and Training, 1991). Many outsiders and even insiders did not immediately notice that the brief ALLP was a fundamentally different policy from the book-length NLP, but Moore convincingly shows that the two were based on uite opposite perceptions of realities, the needs that were derived from and the responses to them. To quote: "the NPL assumed pluralism as a common social good that policymaking on languages would enhance. The ALLP prioritized literacy and 'foreign' languages, using these to displace NLP's commitments." (2000:27). The evolution of the NLP has been described elsewhere (esp. in U. Ozolins, 1993. The politics of language in Australia. Cambridge: CUP), but it is still useful to have her version that pinpoints the major shift from Gough Whitlam to Malcolm Fraser (in 1975). the latter managed (or was forced) to shape a bi-partisan consensus on the respective roles of English and non-English migrant and indigenous languages and on the provision of language services. Equity, social justice and access were the political catch-words of the day. The ALLP, in contrast, emphasized English — mainly in terms of literacy_and the usefulness of foreign, especially Asian languages. Economic rationalism, market principles, cost-efficiency, national frameworks and performance, (later) outcome-based evaluation in the education system were its key words. Moore adds that "[T]he new policy regime had no place for seeking consensus with anyone outside the alliance who had constructed its virtual realities. The ALLP announced to anyone working within the NLP's assumptions that their concerns were important only insofar as they could be co-opted into the goals constructed" (2000:45). That policy came, it should be added, from Labor governments and the current Coalition did not have to do much to accentuate the shift to economic and national-benefit concerns. It looks as if Australia's language policy has been reduced to one on the teaching of English and foreign languages, though one should add in fairness that other concerns have remained unchallenged. To mention ethnic radio and print media, the broadening of foreign language choices for the General School Certificate, etc., the boost to the language teaching industry, and the possibility of language maintenance efforts. Moore's discussion on which theoretical model on language planning is better to explain the Australian data is lengthy, though useful for the specialist. 
Without having gone into details on other contributions, I should say that the Ricento's book is a valuable contribution to the field and appropriate not only for courses on language planning and global English, but also for a language policy angle of Australian Studies that deals with societal aspects of languages. 
Ager, Dennis, 2001. Motivation in language planning and language policy. Clevedon, UK: Multilingual Matters, pp vi+210. ISSN 1-85359-529-2 (hbk), 1-85359-528-4 (pbk). Rezensiert von Gerhard Leitner, Freie Universität Berlin

Language planning, policy and implementation is a growth area and Australia is increasingly a part of it. Ager is no exception to that though Australia only covers a few pages. The book is of particular interest with its focus on the motivation behind policy and planning instead of on details on codification and the mechanisms of policy formulation. Ager proposes an ambitious theory that integrates socio-political, socio-psyhological and linguistic aspects. The Introduction provides a lucid starting point that is further developed in chapters 7 to 10. A series of cases and general themes are deiscussed in detail in chapters 1 to 6 . The overall structure shows the book's interest in theoretical issues in planning and policy. Chapter 6 deals with Australia but some of the theory is essential to an understanding of what he says about it.

The Introduction clarifies some theoretical concepts and proposes the skeleton of a theory. Thus, he distinguishes between language planning and policy, with the former being reserved for "the unofficial influence exercised by individuals and communities" (p 6) and for actions of "organized communities, united by religious, ethnic or political ties" (p 5) in relation to the ways members use their language(s), the practices in education or the codifying efforts by academies and other bodies. The term 'policy' is reserved for the official influence of governments. But for policy to become effective there must be planning and Ager fails to convince the reviewer of the need to make that distinction. In line with common practice, he identifies the planning of the status, the corpus (or expressions) and the acquisition of a language. Motivation, i.e. the reasoning that incites individuals, groups or nations to involve themselves in policy and planning, is a composite concept that consists of goals, attitudes and motives. Of the latter, there are identity (itself), ideology, image, insecurity, inequality, integration and instrumentality, which are are connected by an identity sequence ( $\mathrm{p}$ 135ff). On that he says that organisms are constructing (i) an identity and (ii) an image of it that is (iii) evaluated against the reality around it. The outcomes of stage (iii) determine which steps occur subsquently. There can be (iv) the promotion of a (positive) image, (v) an awareness of insecurity that needs be redressed, (v) an inquality of resource allocation that must be rectified, (vi) a need to defend one's identity, etc. The status of the identity sequence remains unclear. Is it a temporal one? Is it a socio-psychological one? Is it mere positions on a scale that one can move (linearily?) up and down? It would seem that this would make most sense in light of the conflicting positions that can be taken by actors in planning and policy. Ager deals with three types of agents, viz. individuals, groups and nations, but they are seen in isolation and he is not concerned with how the transition from individual to group and on to nation works. Incidentally, it might be of interest to look at the Teun van Dijk's recent cognitive work on the transimission of ideology from a media to individuals and groups ("Opinions and ideologies in the press", in: Allan Bell, Peter Garrett, eds, Media Discourse. Oxford: Blackwell. 21-63). That would help bridge the argumentative gap in Ager's theory. I have omitted from this account his comments on goals/ends and attitudes-the other ingredients of motivation, though the reader can expect highly interesting analyses. It is the motives that provide the headlines to the case studies in chapters 1 to 6 .

These six chapters illustrate the role of motives in various nations or in relation to certain general themes that have been addressed in planning: "The politics of identity have marked the last quarter of the twentieth century with a degree of violence and horror which is unprecedented" (2001:13). He points to former Yugoslavia and the Soviet Union, but discusses the role of identity in France, Algeria, Catalonia, India and Wales. Under the heading of ideology he looks at language and education policies in the UK, which is very useful analysis in view of the fact that Australian (and New Zealand) policies follow closely the British pattern. Germany, Japan and the European Union are subsumed under the motive of image, while insecurity - a motive close to fears and aspirations of ethnic communities - is illustrated with the Gypsies in Central Europe and the French opposition to English. Inequality divides into three levels of severity, viz. inequality, inequity and injustice, and is used to highlight the discrimination suffered by women, the powerless minorities in the European 
Union and by ethnic groups. Gender reform, the European Charter for Regional and Minority Languages and Australia's language policies, thus, form the core of this chapter. Integration and instrumental motives are exemplified with immigrants in the USA, lingua francas in Africa, esp. the use of English, and a typology of individuals acquiring a new language.

Australia is dealt with from the angle of policy and as an instance of the motive of inequality. Its policies were, as he puts it, an attempt to rectify injustice. Explicit government policies took shape in the 1980s only, but he re-iterates briefly the periods of implicit policies from the beginning of the colony in 1788, which, as I said above, bear a close resemblance with Britain. Like Clyne, he believes that there was a practical recognition or toleration of linguistic pluralism up to World War I. But he makes the important points that practical action amounted to ignoring all languages other than English (p. 96) and that there was a shift at around World War I when those languages were explicitly banned and when policy created linguistic injustice. Ager fails to integrate that shift into his theory; though the motive that springs to mind is insecurity (cf. chapter 4). Australia, then, went through a period of soul-searching. With Britain being under the threat of a war, Australia had to stay loyal despite the fact that statehood would have required an emphasis on nation-building. A change in policy occurred in the 1980s as a part of a trend towards the acceptance of ethnic, linguistic and cultural pluralism. "Australia", he says, "was now becoming conscious of its own identity and of its differences from Britain." (p. 97); it was now less difficult to pronounce one's background. There was a climate of intellectual conflict with opposition coming from many quarters. But a strong lobby united language professionals, migrant and indigenous groups who were able to push government towards an explicit and coherent language policy. A first document by Lo Bianco (1987) put the emphasis on community needs and aspirations, social equity and linguistic rights, while the government's revision in 1991 emphasized national benefits and the catering for national needs. The motive of rectifying injustice was gradually replaced by the one of '(positive) image' - as I would see it. Ager himself says this on the predominant motives:

"They are (at least) three: concern to accept immigrant communities by giving greater recognition to their diversity...; concern to unite society by ensuring access to power, but through the use of one 'unifying' language, English; and an economic motivation aimed at ensuring that Australia's situation as part of Asia and the Pacific rim was recognised... Whether the correction of inequality and injustice was foremost in the minds of politicians is somewhat doubtful..." (p. 101)

The motive of (rectifying) injustice, which was argued for by the lobby groups, was defeated by that of ideology, viz. the projection of a positive image into the geo-political region, I would suggest. Ager notes that a distinction should be made between non-English migrant and Aboriginal languages. The prime motive regarding non-English migrant languages was to clarify certain rights (such as the provision of language services), while Aboriginal languages continued to be promoted to rectify an injustice. What is interesting in his account is the fact that diverse motives, held by overlapping sets of communities, brought about a unique result, viz. an explicit policy in an Anglophone country. It also emerges that it is doubtful whether one can or should deal with the motivation of individuals, communities and states in the same way. Individuals, it would seem, strive for a greater degree of coherence in their individual and social identity, while the state is a domain of conflictings. He also implies that it is not easy to distinguish policy from planning and between motives, goals and attitudes.

Critical comments apart, Ager's book is a worthwhile study of language policy. It develops an approach that is able to relate language policy with planning at the level of social interaction. Even if it is not specifically about Australia, Australianists can benefit from the contextualization of Australia in a general theory and the comparisons that are possible with those countries that have implicit and the few that have implicit policies. 


\section{Dümling, Albrecht, Hsg., 2000. Zu den Antipoden vertrieben. Das Australienexil deutsch- australischer Musiker. Saabrücken: Pfau Verlag. Rezensiert von Manfred Brusten, Universität Wuppertal}

Ein wirklich interessantes Buch, das Albrecht Dümling im Auftrag von musica reanimata - dem Förderverein zur Wiederentdeckung NS-verfolgter Komponisten und ihrer Werke herausgegeben hat.

Wer jedoch aufgrund des Titels glaubt, daß es sich hier lediglich um eine Fachpublikation von und für Musikwissenschaftler handelt, irrt. Das Buch ist eher eine echte Fundgrube für alle, die ein Interesse an Australien haben. Neben einer Einleitung des Herausgebers umfaßt der Sammelband auf insgesamt ca. 160 Seiten ein buntes, bestens aufeinander abgestimmtes Kaleidoskop von 15 Kurzbeiträgen, in denen sich zehn Autoren unterschiedlicher Fachprominenz mit Problemen und Fragen befassen, die die Situation deutschsprachiger Musiker analysieren, die infolge der Judenverfolgung während des Dritten Reiches nach Australien ins Exil vertrieben wurden.

In der Kürze liegt die Würze: Hier kommt der wahre Kern dieses Sprichwortes voll zur Geltung. In welchen Beitrag der Leser auch hineinliest - er wird ihn nicht mehr zur Seite legen, bevor er ihn zu ende gelesen hat; und das nicht nur wegen der bemerkenswerten Kurzweiligkeit der Beiträge, sondern auch wegen ihrer ,message'.

Alfred Dümling berichtet in seiner Einleitung zunächst über die Entstehungsgeschichte eines Kolloquiums, das unter dem Thema ,Musiker-Exil in Australien“ vom 3.-5. Mai 1996 im Dresdner Zentrum für zeitgenössische Musik stattfand zum Ausgangsereignis des nunmehr vorliegenden Sammelbandes wurde.

Im ersten Kapitel befassen sich zwei Beiträge mit Australien als Exilland:

Johannes H. Voigt, Professor für Überseegeschichte, gibt zunächst einen komprimierten historischen Überblick über die Auswanderung von Deutschen nach Australien - von 1838 (der ersten großen deutschen Migrationswelle von Altlutheranern nach Südaustralien auf der Flucht vor dem Preußischen Staat) bis zu den Migranten, die nach dem Zweiten Weltkrieg zu Hunderttausenden - als Displaced Persons, Überlebende zerbomter Städte und verlorener Schlachten, heimatlos gewordene NS-Verfolgte, Flüchtlinge und Vertriebene sowie Juden, die den staatlich organisierten Holocaust überlebt hatten - nach Australien strömten und dort - nach Ansicht der australischen Regierung - helfen sollten, einen Damm gegen die befürchtete ,Flut ' aus Asien aufzubauen; unter ihnen bis zu Beginn der 80er Jahre allein ca. 160.000 Deutsche. Dabei bildete die Musik dieser deutschen Auswanderer und Flüchtlinge - ihr religiöses und volkstümliches Liedgut, die Organisation von Liedertafeln und die Vermittlung klassischer europäischer Musiktraditionen (Opern, Symphonien, Konzerte) - nicht nur ein zentrales Element ihrer eigenen Identität und eine Brücke zur verlassenen Heimat, sondern - wenn auch politisch nicht gerade beabsichtigt - zugleich einen wesentlichen Beitrag zur multikulturellen Gesellschaft des heutigen Australien.

Albrecht Dümling schließt mit seinem musikwissenschaftlichen Überblick über ,Berliner Musiker in Australien" nahtlos und ergänzend an. Im Vordergrund stehen bei ihm vor allem einige der herausragenden Persönlichkeiten der deutsch-australischen Musikgeschichte. Unter ihnen der aus Berlin stammende Komponist Carl Linger, der als Anhänger der bürgerlichen Revolution von 1848 mit dreihundert weiteren deutschen Auswanderern im August 1949 nach viermonatiger (!) Schiffsreise in Port Adelaide eintraf und 1859 für den von ihm komponierten patriotischen, Song of Australia' den ersten Preis erhielt. Goldfunde in Victoria und der damit verbundene Zustrom von Abenteurern führten nicht nur zu einem neuen Reichtum des Landes mit prachtvollen Straßen in Melbourne, sondern auch zum Aufbau einer respektablen Musikkultur, bei dem deutsche Einwanderer trotz der britischen Grundorientierung wichtige Akzente setzten; unter ihnen Julius Herz, der 1882 mit dem ,Melbourne Musical Festival ' das erste Musikfest auf dem australischen Kontinent organisierte. Ein halbes Jahrhundert später - und danach - wird das deutsche Musikleben 
in Australien immer stärker durch Exilanten geprägt, die dem Naziterror in Deutschland und Österreich entfliehen konnten; unter ihnen befindet sich der 1928 in Wuppertal geborene und heute wohl bekannteste deutsch-australische Komponist George Dreyfus, der im Sommer 1939 mit einem Kindertransport in Melbourne eintraf, und der 1922 in Berlin geborene Komponist und Musikkritiker Felix Werder, der ein Jahr später mit dem britischen Truppentransporter ,Dunera " von Southampton nach Sydney deportiert und dann als ,feindlicher Ausländer ${ }^{6}$ zunächst einmal entbehrungsreiche Jahre im Internierungslager Tatura verbringen mußte.

Das zweite Kapitel enthält vier Beiträge, die sich mit der Deportation deutscher und österreichischer Juden befassen, die vor dem staatlich organisierten Terror nach England geflohen waren, und dann im Sommer 1940 - angesichts einer befürchteten Invasion deutscher Truppen und einer Massenhysterie gegen alle Fremden im Lande, die sich als ,fünfte Kolonne' betätigen könnten nach Australien verschifft und dort in den Lagern von Hay und Tatura interniert wurden.

Die historischen und weltpolitischen Hintergründe für diese Deportation und Internierung deutscher Juden als ,Auftakt zum Exil in Australien“ schildert sehr anschaulich der hervorragende KurzBeitrag von Wolfgang Benz, Leiter des Zentrums für Antisemitismusforschung an der TU Berlin und Vorsitzender der Gesellschaft für Exilforschung. Im Anschluß an seinen Beitrag - erstmals in Deutschland veröffentlicht - die offizielle Liste der auf der ,Dunera' Deportierten.

Walter Kaufmann, 1924 in Berlin geboren, nach der Deportation seiner Eltern ins KZ Theresienstadt nach England emigriert, ist einer der sogenannten ,Dunera Boys', die 1940 von den Engländern nach Australien verfrachtet wurde, jedoch später nach Europa zurückkehrte, um in der DDR als Schriftsteller ein , besseres Deutschland“ aufzubauen. Wer könnte besser geeignet sein für eine autobiographische Shortstory über die merkwürdige Zugreise vom Ankerplatz der ,Dunera ' im Hafen von Sydney bis ins staubig-heiße Internierungslager von ,Hay` am Rande des australischen Wüste?

Klaus Loewald, 1920 in Berlin geboren, erinnert in einem sehr persönlichen Beitrag zum ,Musikleben in den australischen Internierungslagern' an den aus Wien stammenden Berufspianisten Peter Stadlen, der - ebenfalls mit der ,Dunera' nach Australien verschifft und dort in den Lagern von Hay, Orange und Tatura hinter Stacheldraht fast eineinhalb Jahre lang festgehalten - neben anderen Kulturschaffenden in den Lagern wichtige und z.T. überwältigende Beiträge zum kulturellen Leben (und Überleben) der dort internierten Flüchtlinge aus Europa leistete: Solokonzerte mit Musik von Beethoven, Schubert, Chopin und Strawinsky und schließlich sogar einen Männerchor aus Internierten regelrecht, aus der Wüste stampfte'. Der Beitrag von Klaus Loewald wird ergänzt durch Auszüge aus einem längeren autobiographischen Interview mit ihm: Erinnerungen und Kommentare über seine jüdische Familie in Berlin, Verfolgung durch die Nazis, Emigration nach England, Erlebnisse auf der ,Dunera' und in den australischen Internierungslagern, Emigration nach USA, Studium der Politologie und Geschichte in Berkeley und Berlin und schließlich US-amerikanischer Kulturattachée in Vietnam und Australien.

Das dritte Kapitel ist ausschließlich den beiden bereits genannten ,Komponisten im Exil` - Georg Dreyfus und Felix Werder - vorbehalten.

Zur Einführung biographische Anmerkungen von Kay Dreyfus zu einem ,Porträtkonzert ' von und mit George Dreyfus, das einige seiner bekanntesten Werke umfaßt: ,Larino, Safe Haven“, (1989), ,Sextett für Didjeridu und Bläserquintett` (1971) und Beispiele seiner Filmmusik: ,Rush“ (einprägsame Titelmusik einer geradezu klassischen australischen Abenteuer-Fernsehserie (1974) und die ,Adventures of Sebastian the Fox ‘ (aus einer Fernsehserie für Kinder, 1963).

In seinen eigenen weitgehend aphoristisch-sarkastischen Beitrag setzt sich George Dreyfus anschließend in einem für ihn charakteristischen deutsch-australischen Vortragsstil mit seinem eigenen musikalischen Exil in Australien auseinander, das - für ihn - ,wahrscheinlich nie ein solches war". 
Auch die Werke des zweiten ,Komponisten - und Musikkritikers - im Exil‘, Felix Werder, den der russisch-jüdische Komponist Larry Sitsky (Canberra) als den ,bei weitem profiliertesten australischen Komponisten" bezeichnet, wird zunächst mit Anmerkungen zu seinem ,Portraitkonzert“ vorgestellt. Seine Musik - ein wahres Kontrastprogramm zu George Dreyfus -, basiert - so Sitsky - auf der philosophischen Erkenntnis der immensen Traditionen europäischer Kunst und europäischen Denkens. Eine Einschätzung, die Felix Werder durch seinen eigenen Beitrag über „German Jewish Expressionism“ trefflich bestätigt.

Das vierte und letzte Kapitel bietet ein Kolloqium über ,Perspektiven der Exilforschung :

Der Soziologe und Musikwissenschaftler Alphons Silbermann, der selbst - auf der Flucht vor den deutschen Nazis - etliche Jahre in Australien mit gefälschtem Zeugnis als Koch verbrachte, beschäftigt sich auf der Basis eigener Erfahrungen mit dem ,Einfluß deutschprachiger Emigranten auf das Musikleben Australiens'. Dort - so erinnert er - seien damals keine Intellektuellen, Freiberufler, Ärzte, Juristen, Künstler oder gar Musiker, sondern nur Handwerker und gelernte Arbeiter erwünscht gewesen. Dabei dürfe vor allem dreierlei nicht übersehen werden: erstens, die jüdischen Komponisten und Interpreten seien aus Deutschland vertriebene Exilanten gewesen und nicht Emigranten, weil sie ihr Heimatland nicht freiwillig verlassen hätten; zweitens, sie hätten ihren Lebensunterhalt nur in außermusikalischen Berufen verdienen können; und drittens, die Tradition und Kultur des Musiklebens Australiens sei damals durch und durch britisch dominiert gewesen, hätte also voll und ganz unter der kulturellen Vormundschaft Englands gestanden. Und last but not least - die sozialen und kulturellen gesellschaftlichen Kontakte zwischen Australiern und jenen, noch mit dem Stigma des Fremden versehenen ,bloody refos 'seien eher selten gewesen.

Für Andrew McCredie, 1977 erster australischer Musikwissenschaftler mit eigenem Lehrstuhl in Adelaide, sind die euro-judäischen Exilmusiker und -komponisten in Australien vor allem ein wichtiger Gegenstand weiterer Forschungen. In seinem äußerst komprimierten und detailreichen musikwissenschaftlichen Beitrag erinnert er an die Bedeutung der bereits 1945/46 entstandenen ,Musica Viva Australia‘ und an neuere Pläne zum Aufbau eines Archivs zur Erforschung der jüdischen Migrantenmusik in Australien. Zentrale Aufgabe dieser von McCredie geforderten Exilforschung ist vor allem die Untersuchung der Probleme, Chancen und Entwicklungen der Kulturtransplantation von einer soziokulturellen Welt in eine andere und damit präzisere Forschungen zu kulturellen Transfers, Prozessen und Phasen der Akkulturation, Enkulturation und der Wechselwirkungen.

Hanns-Werner Heister, seit 1998 Professor an der Musikhochschule Hamburg, befaßt sich unter dem Titel ,Auswanderung versus Flucht“ mit revisionistischen Tendenzen in der Exilforschung und wendet sich dabei vehement gegen die Verharmlosung und Nivellierung der Differenz von ,Exil` zu ,Emigration“. Auf dem Exilbegriff zu beharren ist daher für ihn ein Beitrag zur wissenschaftlichen Wahrheit, um der Verharmlosung des Zwangs vorzubeugen, der zum oft lebensnotwendigen und lebensrettenden Weg ins Exil führte. Denn der Begriff des Exils umfasse - im Gegensatz zu dem der Emigration - einerseits den politisch vermittelten Zwang zum Verlassen der ursprünglichen Heimat, andererseits die - zumindest meist zunächst - beabsichtigte Rückkehr. So ließe sich speziell im Hinblick auf die USA - deutlich erkennen, wie der Emigrationsbegriff (bzw. der Begriff der Auswanderung) immer wieder politisch für die Ideologie des US-amerikanischen ,melting pots“ instrumentalisiert werde und dadurch ein problematischer Begriff mit ideologischer Schlagseite geworden sei; und der damit - nicht zuletzt auch und vor allem mit Bezug auf die Judenverfolgung während des ,Dritten Reiches ‘ - zu einer unverantwortlichen revisionistischen Verharmlosung des Nationalsozialismus selbst beitrage und den Gedanken an eine Einladung zur Rückkehr der Exilanten - und sei es via ihres Werkes - gar nicht erst aufkommen ließe.

Auch Gerhard Stilz, Professor für englischsprachige Literatur in Tübingen, beklagt abschließend als ,Außenseiter ‘ - in seinen ,Randbemerkungen zur Erforschung des literarischen Exils in Australien“ ebenfalls die irritierende Unschärfe in der Verwendung der Begriffe ,Exil` und ,Emigration‘, wobei auch für Stilz der Begriff ,Exil‘ vor allem erzwungene Auswanderung, 
Deportation und Internierung impliziert und die sich daran anschließende Entfremdung von einer räumlich entfernten ,Heimat“. Mehr noch - Stilz' Literaturdurchsicht läßt den Schluß zu, daß Australien in der internationalen Exilforschung über lange Zeit fast keine Rolle gespielt habe und auch die Spezialliteratur zum Exil in Australien (speziell ihre Repräsentanz in Deutschen Bibliotheken) in Umfang und Aufmachung eher bescheiden geblieben sei. Aber selbst wenn man sich bemühen würde, dieses Defizit zu verringern, bliebe weitgehend unklar und strittig, was denn überhaupt als Exilliteratur oder Exilmusik zu gelten habe und wer zu jenen gehöre, die als Exilanten zu bezeichnen wären.

Fragen über Fragen und tausend kontroverse Antworten. Kein Zweifel: Die Forderung nach mehr Exilforschung - vor allem auch in und über Australien - kann nur begrüßt werden. Der vorliegende, von Albrecht Dümling herausgegebene Sammelband gibt hierzu eine ganze Reihe wichtiger Anstöße. 